\title{
FLUID FLOW CHARACTERIZATION AND IN SILICO VALIDATION IN A RAPID PROTOTYPED ABDOMINAL AORTIC ANEURYSM MODEL
}

\author{
A Thesis \\ presented to \\ the Faculty of California Polytechnic State University, \\ San Luis Obispo
}

\author{
In Partial Fulfillment \\ of the Requirements for the Degree \\ Master of Science in Biomedical Engineering
}

by

Dean Wampler

March 2017 
(C)2017

Dean Thomas Wampler

ALL RIGHTS RESERVED 


\section{COMMITTEE MEMBERSHIP}

TITLE:

Fluid Flow Characterization and In Silico Validation in a

Rapid Prototyped Abdominal Aortic Aneurysm Model
AUTHOR:
Dean Thomas Wampler

DATE SUBMITTED: $\quad$ March 2017

COMMITEE CHAIR: $\quad$ David Clague, Ph.D.

Professor of Biomedical and General Engineering

COMMITTEE MEMBER: Lily Laiho, Ph.D.

Professor of Biomedical and General Engineering

COMMITTEE MEMBER: Dan Walsh, Ph.D.

Professor Emeritus of Biomedical and General Engineering 


\begin{abstract}
Fluid Flow Characterization and In Silico Validation in a Rapid Prototyped Abdominal Aortic Aneurysm Model

Dean Thomas Wampler
\end{abstract}

Aortic aneurysms are the $14^{\text {th }}$ leading cause of death in the United States. Annually, abdominal aortic aneurysm (AAA) ruptures are responsible for 4500 deaths. There are another 45,000 repair procedures performed to prevent rupture, and of these approximately 1400 lead to deaths. With proper detection, the aneurysm may be treated using endovascular aneurysm repair (EVAR). Understanding how the flow of the blood within the artery is affected by the aneurysm is important in determining the growth of the aneurysm, as well as how to properly treat the aneurysm. The goal of this project was to develop a physical construct of the AAA, and use this construct to validate a computational model of the same aneurysm through flow visualization. The hypothesis was that the fluid velocities within the physical construct would accurately mimic the fluid velocities used in the computational model. The physical model was created from a CT scan of an AAA using 3D printing and polymer casting. The result was a translucent box containing a region in the shape of the aneurysm. Fluid was pumped through the construct to visualize and quantify the velocity of the fluid within the aneurysm.

COMSOL Multiphysics® was used to create a computational model of the same aneurysm, as well as obtain velocity measurements to statistically compare to those from the physical construct. There was no significant difference between the velocity values for the physical construct and the COMSOL Multiphysics ${ }^{\circledR}$ model, confirming the 
hypothesis. This study used a CT scan to create an anatomically accurate model of an AAA that was used to validate a computational model using a novel technique of flow visualization. As EVAR technologies continue to progress, it will become increasingly important to understand how the blood flow within the aneurysm affects the growth and treatment of AAAs.

Keywords: Abdominal aortic aneurysm, computational validation, COMSOL Multiphysics ${ }^{\circledR}, 3 \mathrm{D}$ printing, PDMS mold 


\section{ACKNOWLEDGMENTS}

Thank you to:

i) My advisor, Dr. Clague, for introducing me to this project and supporting me throughout the process

ii) Leah Ashley and Daniel Vogel for helping me with some of the details of this project

iii) Daniel Greinke, Rachel Willis, and Alexandra Knauer for allowing me to further develop their flow visualization concepts, as well as providing elaboration of their methods

iv) Dylan Sigley for allowing me the use of his 3D printer as well as material

v) My parents for supporting me throughout the entire process 


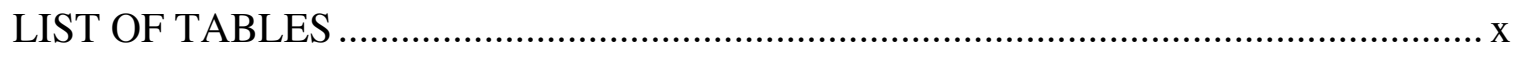

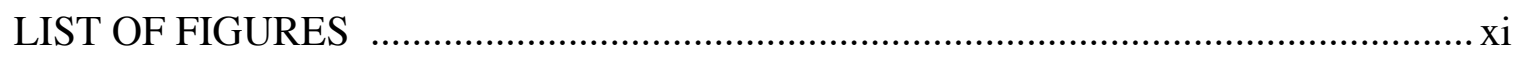

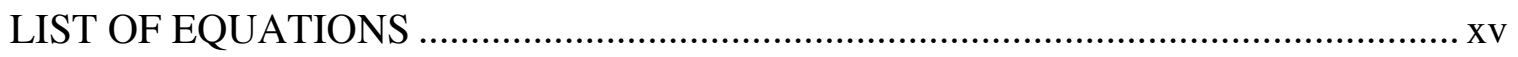

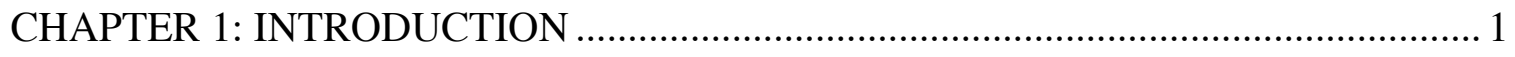

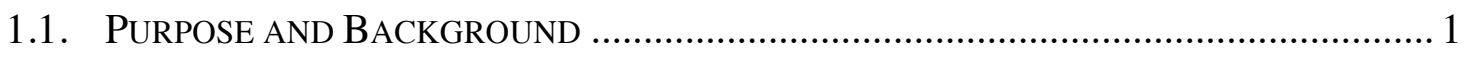

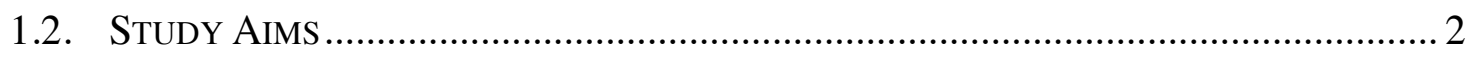

CHAPTER 2: BACKGROUND AND RELATED STUDIES ……………………....... 4

2.1. NEEDS FOR MODEL AND EXPERIMENTS ……………......................................... 4

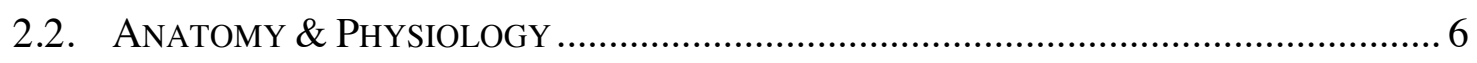

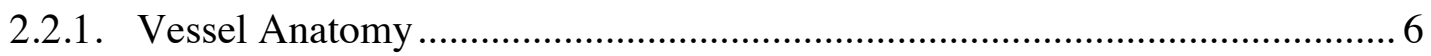

2.2.2. Physiology of the Aneurysm .............................................................. 7

2.2.3. Blood cells and Platelets .......................................................................... 8

2.2.4. Normal Physiological Values ................................................................... 9

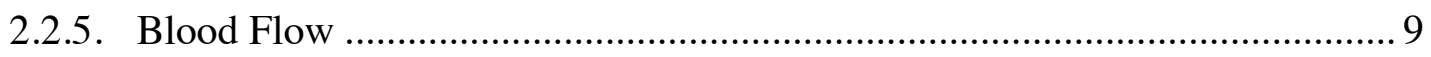

2.2.6. Wall Shear Stress and Blood flow ............................................................ 9

2.2.7. Shear Stress Physiology ………………………...................................... 14

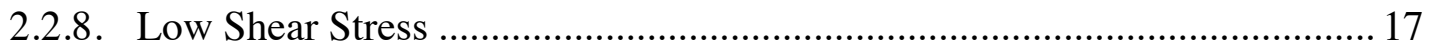

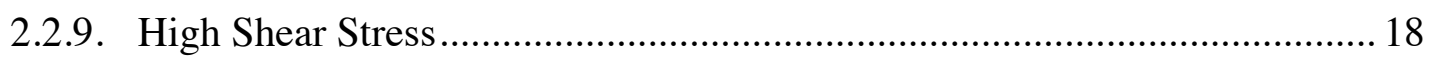

2.2.10. Reynolds Number and the Effect on Blood Flow ......................................... 19

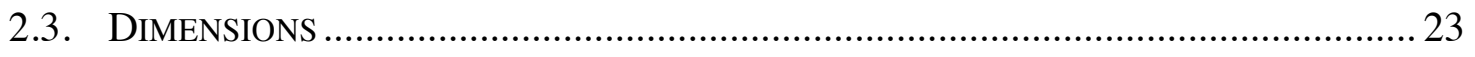

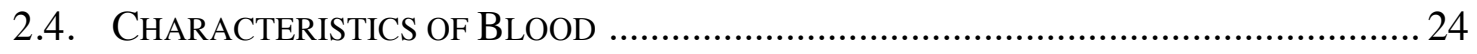




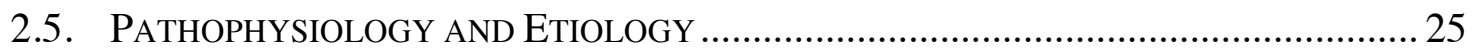

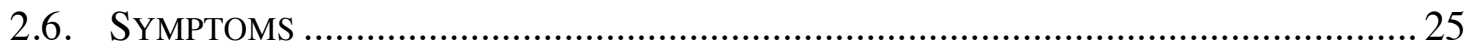

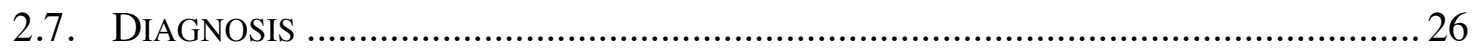

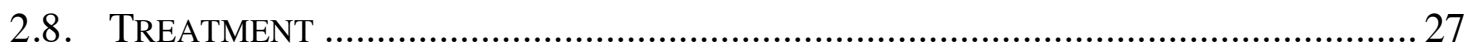

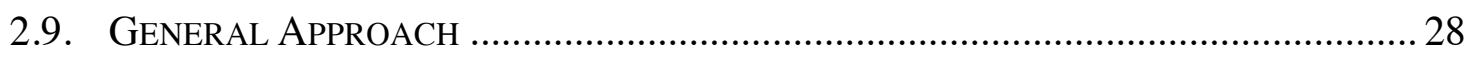

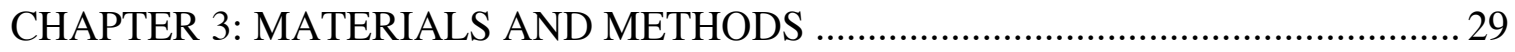

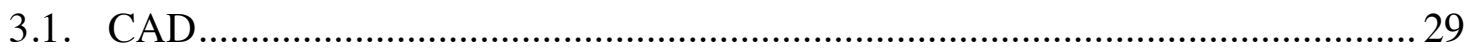

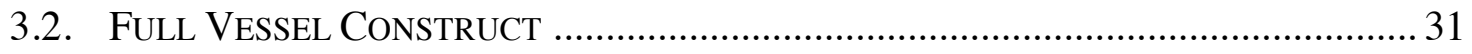

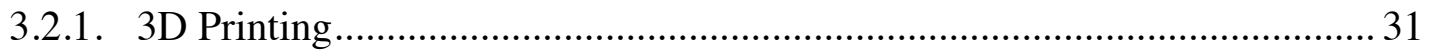

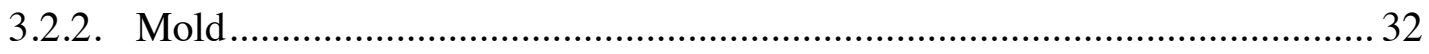

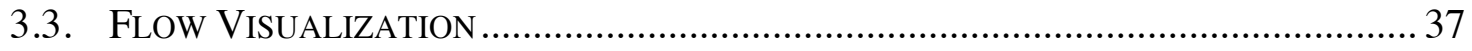

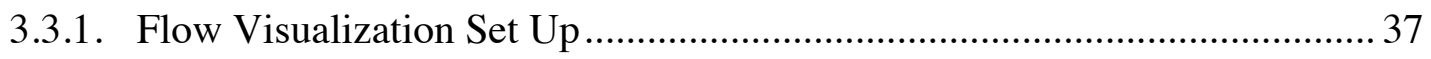

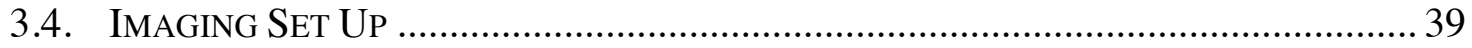

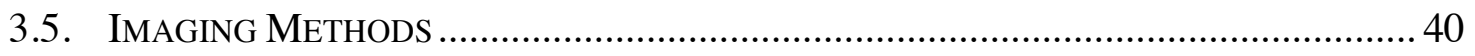

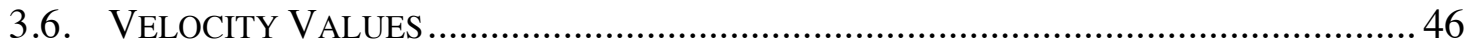

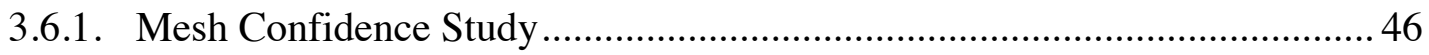

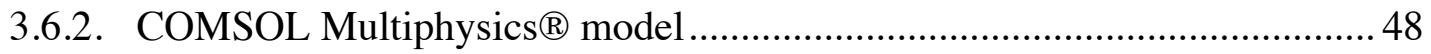

3.6.3. COMSOL Multiphysics ${ }^{\circledR}$ Validation using normal blood vessel ................. 49

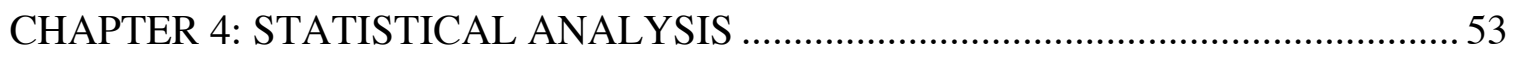

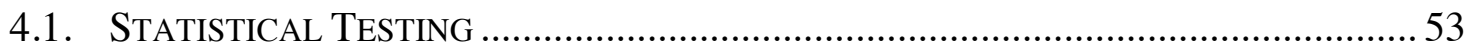

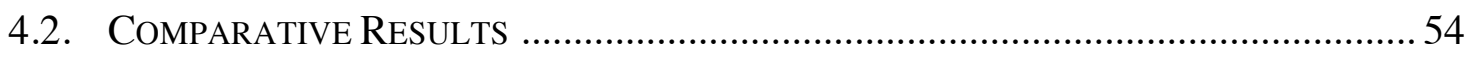

CHAPTER 5: DISCUSSION AND RESULTS ............................................................ 57

5.1. INTERPRETATION OF EXPERIMENTAL MODEL ....................................................... 57 
5.2. INTERPRETATION OF COMSOL MULTIPHYSICS $®$ MODEL AND COMPARISON STUDY 58

5.3. FDA USE OF COMPUTATIONAL MODELS 62

CHAPTER 6: SUMMARY AND CONCLUSIONS 64

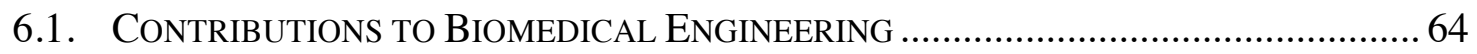

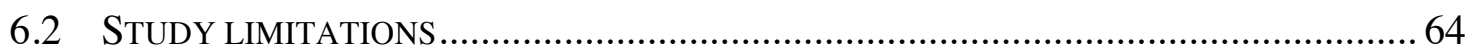

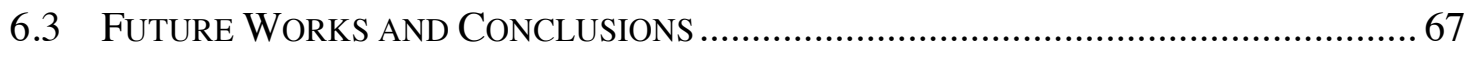

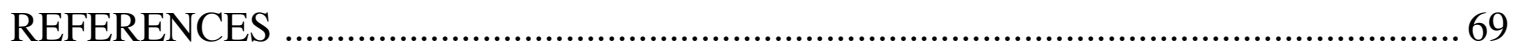

APPENDICES

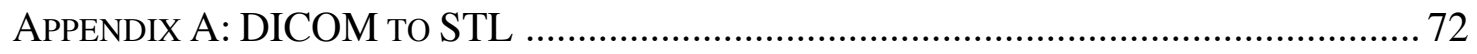

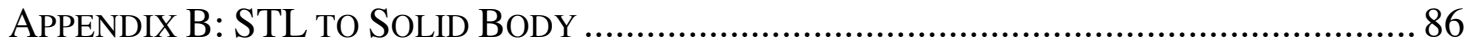

APPENDIX C: REMOVING UNNECESSARY ASPECTS OF AAA MODEL .............................. 94

APPENDix D: Reducing the Number of FACES ON AAA Model ……....................... 105

APPENDIX E: SCALING THE AAA Model ............................................................... 111

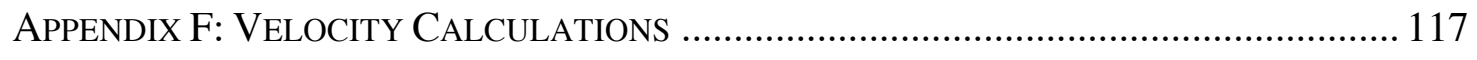

APPENDIX G: FLOW VISUALIZATION CONVERSION................................................... 118

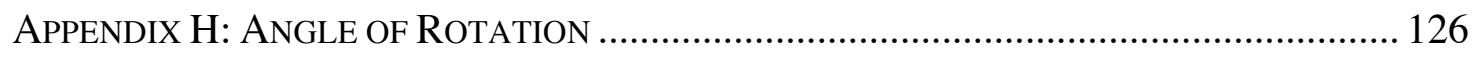

APPENDIX I: COORDINATE CONVERSION …………….......................................... 130

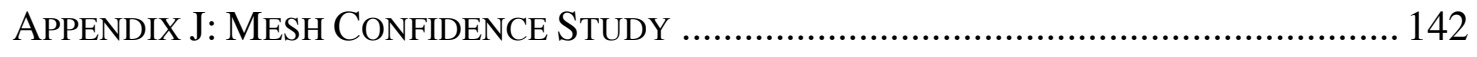

APPENDIX K: COMSOL MULTIPHYSICS ${ }^{\circledR}$ SETUP ………………………………...... 143

APPENDIX L: MESH STATISTICS FOR COMSOL MULTIPHYSICS ${ }^{\circledR}$ VALIDATION STUDY 
Table 1. COMSOL Multiphysics Mesh levels and corresponding number of elements

Table 2. Velocity Values used in the Mesh Confidence Study ........................................ 47

Table 3. Percent differences of the velocity values used in the Mesh Confidence Study

Table 4. Given information for COMSOL Multiphysics ${ }^{\circledR}$ validation study ..................... 49

Table 5. Parameters used in COMSOL Multiphysics ${ }^{\circledR}$ validation study .......................... 50 
Figure 1. Anatomy of the aorta from the aortic root to the common iliac artery..... 6

Figure 2. Anatomy of a vessel. Tunica externa (adventitia), tunica media, and tunica intima

Figure 3. Velocity field of blood flow within an artery 11

Figure 4. Bifurcation in an artery and the resulting regions of high shear stress and low shear stress 13

Figure 5. Chemical forces acting on endothelial cells 15

Figure 6. Shear stress acting on endothelial cells 15

Figure 7. Effect of high and low shear stress on the signaling factors of the endothelium

Figure 8 . The correlation coefficients of clinically measurable factors with peak wall stress on the 6 AAAs.

Figure 9. Streamlines for laminar steady flow at (a) $\operatorname{Re}=10$; (b) $\operatorname{Re}=100$; (c) $\operatorname{Re}=500$;

(d) $\operatorname{Re}=1000 ;$ (e) $\operatorname{Re}=1750$; and (f) $\operatorname{Re}=2265$ : The direction of the flow is from left to right. 20

Figure 10. Pressure distribution along the wall of the double-aneurysm model for selected Reynolds numbers 21

Figure 11. Wall pressure, wall shear stress, and streamlines for $\operatorname{Re}=2000$ 22

Figure 12: Pressure and velocity pulse waveforms in the aorta and arterial branches of a dog. The pressure maximum becomes amplified while the velocity maximum decreases as the blood moves downstream. 
Figure 13.3D print of the abdominal aortic aneurysm using PLA.............................. 31

Figure 14. The molding rubber was cut from the 3D printed aneurysm using an X-Acto knife. 33

Figure 15. Rubber aneurysm mold with a hollow chamber into which liquid paraffin wax was poured. 34

Figure 16. The acrylic box and the ingredients used to create the PDMS mold 34

Figure 17. Part A and Part B mixed and in the process of vacuum degassing 35

Figure 18. The Wax model suspended in the acrylic box 35

Figure 19. The Wax model suspended in the acrylic box 35

Figure 20. Side view of the wax inside the PDMS mold 36

Figure 21. Front view of the wax inside the PDMS mold 36

Figure 22. Side view of the hollow PDMS mold ..................................................... 36

Figure 23. Front view of the hollow PDMS mold 36

Figure 24. (a) The tubing connected to the PDMS construct. (b) The set-up of the flow visualization. (c) The side of the PDMS construct where the videos were taken..... 39 Figure 25. Origin of the COMSOL Multiphysics® model (red dot). The coordinate system of the COMSOL Multiphysics ${ }^{\circledR}$ model is located in the lower left corner of the graphics window. 41

Figure 26. The origin of the PDMS construct. The coordinate system is set up with $x=0$, $\mathrm{y}=0$ in the lower left corner of the image. 42

Figure 27. Velocity slice plot of artery for COMSOL Multiphysics ${ }^{\circledR}$ validation. 51

Figure 28. Line maximum used to find the maximum velocity of the artery fluid model 
Figure 29. Shear stress slice plot of artery for COMSOL Multiphysics ${ }^{\circledR}$ validation....... 52

Figure 30. Surface selected to determine the average surface shear stress of the artery

fluid model 52

Figure 31. Paired 2-sample t-test results calculated using JMP software. 53

Figure 32. Scatter plot of Trial 1 comparing the COMSOL Multiphysics ${ }^{\circledR}$ and experimental velocity values at each coordinate point. 54

Figure 33. Scatter plot of Trial 2 comparing the COMSOL Multiphysics ${ }^{\circledR}$ and experimental velocity values at each coordinate point.

Figure 34. Scatter plot of Trial 3 comparing the COMSOL Multiphysics ${ }^{\circledR}$ and experimental velocity values at each coordinate point.

Figure 35. Scatter plot of Trial 4 comparing the COMSOL Multiphysics $\AA^{\circledR}$ and experimental velocity values at each coordinate point.

Figure 36. Wall shear stress in Pa for the aneurysm model plotted in COMSOL Multiphysics®

Figure 37. Zoomed in image of the wall shear stress in the neck of the COMSOL Multiphysics $®$ aneurysm model

Figure 38. Zoomed in image of the wall shear stress in the legs of the COMSOL Multiphysics ${ }^{\circledR}$ aneurysm model

Figure 39. Plot of the wall pressure in Pa on the COMSOL Multiphysics ${ }^{\circledR}$ aneurysm model 60

Figure 40. Zoomed in image of the wall pressure in the legs of the COMSOL Multiphysics $\AA$ aneurysm model. 60 
Figure 41. Wall shear stress values in $\mathrm{Pa}$ in a healthy aorta given the same inlet diameter, viscosity, density, and mass flow rate as the aneurysm model............................. 61

Figure 42. Pressure in $\mathrm{Pa}$ in a healthy aorta given the same inlet diameter, viscosity,

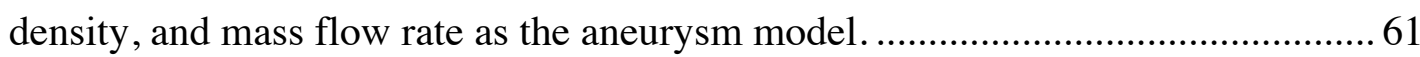




\section{LIST OF EQUATIONS}

Equation 1: $\quad \tau_{w}=\gamma_{w} \mu$

Equation 2: $\quad \tau_{-r z}=-\mu \frac{\mathrm{du}}{d r}$

Equation 3: $\quad \dot{\gamma}_{-r z}=\frac{8 \mu}{d}$

Equation 4: $\quad \tau_{-r z}=8 \mu \frac{u}{d}$

Equation 5: $\quad \tau_{-r z}=32 \mu \frac{Q}{\pi * d^{3}}$

Equation 6: $\quad \dot{\gamma}_{-r z}=\frac{\Delta u}{\Delta r}$

Equation 7: $\quad R e=\frac{\rho v D}{\mu}$

Equation 8: $\quad C O=S V H R$

Equation 9: $\quad \frac{1 L}{15.094 \mathrm{~s}}=\frac{0.264172 \text { gallons }}{15.094 \mathrm{~s}}$ 
Equation 10: $\quad \frac{0.01750178 \text { gallons }}{s}=\frac{63.0064 \text { gallons }}{1 \text { hour }}$

Equation 11: $x_{\text {rot }}=\left[\left(\left(x-C_{x}\right) \cos \theta\right)-\left(\left(y-C_{y}\right) \sin \theta\right)\right]+C_{x}$

Equation 12: $y_{\text {rot }}=\left[\left(\left(x-C_{x}\right) \sin \theta\right)+\left(\left(y-C_{y}\right) \cos \theta\right)\right]+C_{y}$

Equation 13: $\quad Q=\frac{\pi R^{4}\left(P_{1}-P_{2}\right)}{8 \mu L}$

Equation 14: $\quad \tau_{w}=\frac{\left(P_{1}-P_{2}\right) R}{2 L}$ 


\section{CHAPTER 1: INTRODUCTION}

\subsection{Purpose and Background}

Blood flow is an important field of study, both under normal physiological conditions and diseased conditions. The leading cause of death in developed countries relates to cardiovascular disease, of which the majority are related to abnormal blood flow in the arteries [1]. An aneurysm occurs when a part of the wall of an artery weakens, allowing the artery to balloon out. Aortic aneurysms are the $14^{\text {th }}$ leading cause of death in the United States. Annually, AAA ruptures are responsible for 4500 deaths ${ }^{2}$. There are another 45,000 repair procedures performed to prevent rupture, and of these approximately 1400 lead to deaths [2]. Abdominal aortic aneurysm ruptures have been shown to affect 1 in 250 individuals over the age of 50. Men are four times more likely than women to have a ruptured AAA. Five-year survival rate is only $19 \%$, and the overall mortality rate following the rupture of the AAA is over $90 \%$ [3]. 80-90\% of all ruptured aneurysm result in death, however, these deaths may be avoided with proper detection of the aneurysm before it ruptures [4].

With proper detection, the aneurysm may be treated using endovascular aneurysm repair (EVAR). For this procedure, an incision is made in the patient's groin, and a guide wire with an expandable stent graft is passed up the iliac artery to the location of the aneurysm. Once the graft is positioned correctly, the stent graft is expanded within the 
artery. The stent graft pushes against the healthy portion of the artery, and guides the blood through the graft, not allowing it to enter the aneurysm [5].

Understanding how the flow of the blood within the artery is affected by the aneurysm is important in determining the growth of the aneurysm, as well as how to properly treat the aneurysm. The goal of this project is to develop a physical construct of the AAA, and use this construct to validate a computational model of the same aneurysm through flow visualization. As EVAR technologies continue to progress, it will become increasingly important to understand how the blood flow within the aneurysm affects the growth and treatment of AAAs.

\subsection{Study Aims}

Through developing this project, this thesis sought to establish a protocol for three key ideas:

1. Create vasculature with an aneurysm in translucent polymer for flow visualization

2. Make the construct fully three dimensional with no seams

3. Perform flow visualization and velocity characterization for comparison with finite element analysis

The goal of this project was to use a three-dimensional physical model of an AAA to validate a COMSOL Multiphysics ${ }^{\circledR}$ computational model of the blood flow in the 
aneurysm. A three-dimensional model was made to simulate the aneurysm. In this model the velocity of the fluid flow was examined, and was compared to COMSOL Multiphysics ${ }^{\circledR}$ models of the same dimensions and characteristics as the physical model. Success of this project was determined by the ability for the COMSOL Multiphysics® model to reproduce the fluid flow in the physical model. 


\section{CHAPTER 2: BACKGROUND AND RELATED STUDIES}

\subsection{Needs for Model and Experiments}

The purpose for examining and characterizing the shear stress profiles and velocity profiles of an AAA construct was to validate the predicted profiles described by a COMSOL Multiphysics ${ }^{\circledR}$ model. Knowledge of the shear stress and velocity profiles of the aneurysm would allow for more accurate treatment of the aneurysm. A 3D model could be of great use for testing medical devices such as stent grafts. With COMSOL Multiphysics ${ }^{\circledR}$ validation, the 3D model could be used to examine how implants affect the shear stress and velocity profile of the blood flow.

There have been a number of studies performed to investigate AAAs and the role that shear stress and coagulation have on their rupture. One group performed flow visualization studies for steady flow using in-vitro spherical models of aneurysms. They determined the streamline patterns inside the bulge of the models, as well as that a jet of fluid formed inside the aneurysm, surrounded by a region of recirculating fluid [6]. Stehbens et al observed boundary layer separation and reattachment using flow visualization experiments in glass aneurysm models [7]. Drexler and Hoffman improved on experimental models, using asymmetric casting resin models of aneurysms to observe regions of stagnant or reversed flow [8]. Budwig et al examined numerical and experimental studies of steady flow to demonstrate the effect of flow patterns in the pressure distribution and shear stress distribution at the vessel walls [9]. Taylor and 
Yamaguchi demonstrated the existence of two symmetric vortices in three-dimensional computational models using steady flow numerical simulations [10]. Peattie et al observed that the maximum wall pressure inside the aneurysm is located at the center, and that turbulence increases wall shear stresses at the distal end of the bulge $[11,12,13]$. A group proved the existence of monotonic increase of platelet aggregation at the aneurysm wall, reaching a maximum at the distal edge, using a correlation between steady blood flow dynamics and rates of platelet deposition [14, 15].

Studies done by Greinke [16], Willis [17], and Knauer [18] attempted to develop a flow visualization model for vasculature. Greinke and Willis modeled the blood flow in an iliac aneurysm, and Knauer modeled the blood flow in the aortic arch. All three studies were successful in using the aneurysm construct to validate a COMSOL Multiphysics ${ }^{\circledR}$ finite element analysis model. I have taken this previous work and expanded on the idea of using a 3D AAA model to predict flow in-vivo. Using a CT scan of a patient provides realistic geometries for the aneurysm, and through comparison between the PDMS model and the COMSOL Multiphysics ${ }^{\circledR}$ model, I am able to predict shear stresses and flow velocities. The aspects of my thesis that are novel are that I am taking a publicly available CT scan of the AAA and creating a physiologically accurate aneurysm model. The manufacturing of the PDMS construct is unique, in that there were no seams, and the model was a single, solid construct. 


\subsection{Anatomy \& Physiology}

\subsubsection{Vessel Anatomy}

Blood leaves the heart through the pulmonary valve, and travels through the aorta. It then flows through the ascending aorta, aortic arch, descending aorta, and into the abdominal

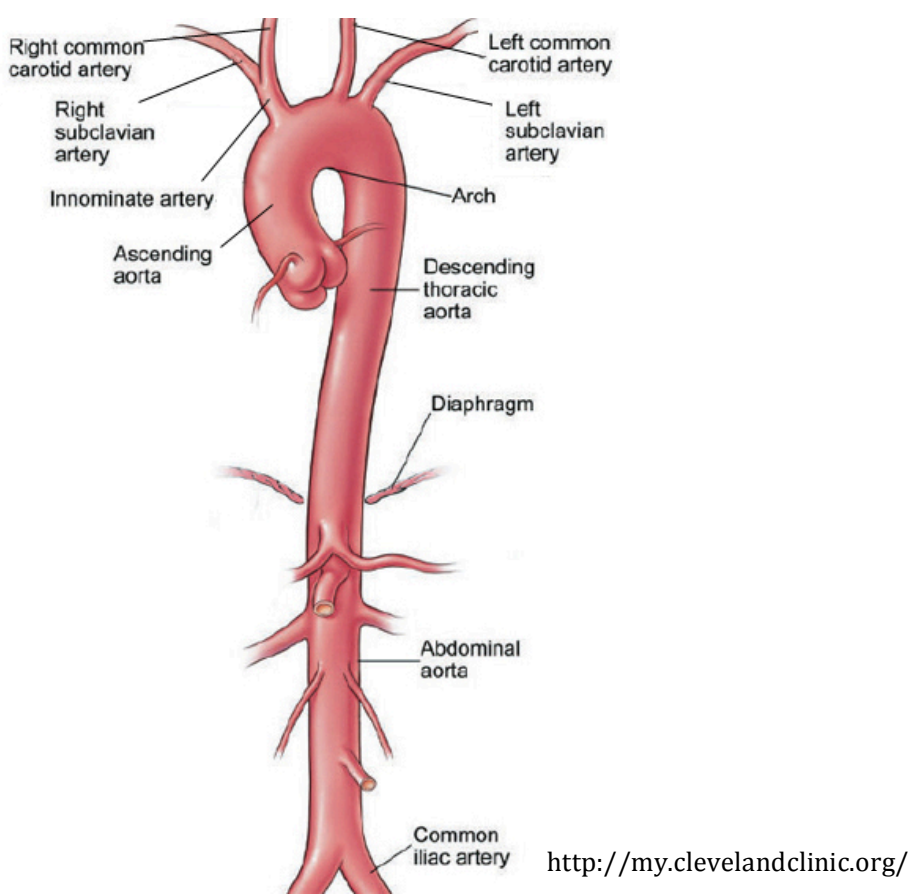

Figure 1. Anatomy of the aorta from the aortic root to the common iliac artery

aorta, from which it splits into the iliac arteries. This anatomy is shown in Figure 1.

The aorta is composed of three tissue layers, the intima, the media, and the adventitia, as

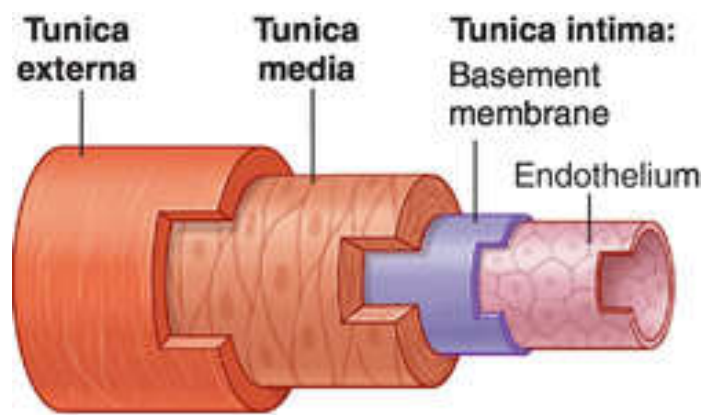

Figure 2. Anatomy of a vessel. Tunica externa (adventitia), tunica media, and tunica intima 
shown in Figure 2. The intima is the endothelial layer. The media consists of smooth muscle cells surrounded by elastin, collagen, and proteoglycans. The elastin is the principal load-bearing element of the aorta. The media is responsible for the structural and elastic properties of the artery. The adventitia is composed primarily of collagen, but also adrenergic nerves and a variety of cells, including fibroblasts and immunomodulatory cells. The endothelial cells on the intima contact blood flow, while the media and adventitia provide mechanical support opposing blood pressure. Tension and deformation in the endothelium opposes the frictional force of the blood (wall shear stress) on the endothelial layer. Circumferential stretch is opposed by circumferential stress and strain in the vessel wall. These forces trigger biochemical reactions that maintain the function of the blood vessels. The cells and fibers can remodel under irregular conditions, such as hypertension. Changes in the chemical signaling can alter the stiffness of the vessel, and change the strain or stress response [19]. Weakening of the artery wall can be a factor leading to the progression of an aneurysm.

\subsubsection{Physiology of the Aneurysm}

In an aortic aneurysm, an increase in the concentration of proteolytic enzymes compared to the concentration of their inhibitors, is thought to degrade the aortic media, particularly as the individual ages. A group investigated the role of metalloproteinases in AAA progression, as studies have shown that patients with AAAs have increased expression and activity of matrix metalloproteinases (MMPs) [20]. MMPs and other proteases are secreted into the extracellular matrix of AAA by macrophages and aortic smooth muscle 
cells (SMCs). MMPs and their inhibitors are responsible for vessel-wall remodeling, and are located in normal aortic tissue. During the development of an aneurysm, the tissue contains increased MMP activity, while the activity of its inhibitors is decreased. This leads to the degradation of elastin and collagen, which are the structural support of the vessel wall. Histology has shown that AAA samples contain lymphocytes and macrophages that may initiate protease activation through various cytokines, including interleukin (IL)-1, IL-6, IL-8, and tumor necrosis factor (TNF) - $\alpha$ [20]. Circulating IL-6 has been shown to be a cardiovascular risk factor. Secretion of IL-6 increases with aortic diameter, which may play a role in inflammation or thrombosis [21], further exacerbating the development of the AAA.

\subsubsection{Blood cells and Platelets}

The characteristics of blood flow are dictated by the components of the blood. Erythrocytes account for nearly half of the blood volume, and are responsible for most of the viscous and elastic properties of blood [22]. The erythrocytes can flow over each other with shear dependent rotational motion. This is a result of the parabolic velocity profile of the flowing blood, as well as the geometry of the red blood cells. This can mix the blood, leading to displacement of proteins and small cells. 


\subsubsection{Normal Physiological Values}

Blood can be modeled as a Newtonian fluid in vessels with a diameter greater than 0.5 mm. A number of boundary conditions exist for the velocity vector. First, there is no slip at the walls (velocity of the fluid at the wall is zero). Second, the velocity profile is symmetric about the centerline of the vessel. Third, the velocity profile is a fully developed parabolic profile at the inlet. Lastly, there is a zero-traction outflow condition at the exit. The molecular viscosity $(\mu)$ of blood can approximated as $\mu=0.00319 \mathrm{~Pa} \cdot \mathrm{s}$, and the density (Q) can be approximated as $1.050 \mathrm{~kg} / \mathrm{m}^{3}[3]$.

\subsubsection{Blood Flow}

Blood flow throughout the majority of the vasculature is laminar, meaning that the viscous forces overpower the relative momentum differences between adjacent layers of fluid [22]. Disturbances between fluid layers are prevented, and a smooth velocity profile is created across the vessel. Blood flow can be considered steady for Reynolds numbers within the range $10 \leq \mathrm{Re} \leq 2265$ [3].

\subsubsection{Wall Shear Stress and Blood flow}

Pressure is created by contractions of the heart, producing hydrostatic force within the blood vessels. Shear stress is thought to only affect the endothelial monolayer, the inner 
surface of the vessel [23]. Blood flow over the endothelium exerts a wall shear force $\left(\tau_{\mathrm{w}}\right)$, which is a frictional drag force per unit area along the wall. $\tau_{\mathrm{w}}$ is related to wall shear stress $\left(\gamma_{\mathrm{w}}\right)$ through the fluid viscosity $(\mu)$ shown in Equation 1 [22].

Equation 1: $\quad \tau_{w}=\gamma_{w} \mu$

The wall shear stress in Equation 1 describes the velocity gradient, and can be rewritten as seen in Equation 2. Here $\mu$ is the kinetic viscosity, $\mathrm{u}$ is the fluid velocity, $\mathrm{y}$ is the distance from the surface, and du/dr is the velocity gradient (shear rate $\gamma$ ) [24]. $\gamma_{\mathrm{w}}$ values typically range from 10 to $50 \mathrm{dyne} / \mathrm{cm}^{2}$ for arterial flow [22].

Equation 2: $\quad \tau_{-r z}=-\mu \frac{\mathrm{du}}{d r}$

Blood typically flows through the vessels with a parabolic velocity field (Figure 3). The blood accelerates as it passes through a decreased vessel diameter, as a result of thrombosis or stenosis. This results in higher $\gamma_{\mathrm{w}}$ values in the region of reduced diameter. As the blood passes the region of reduced diameter and reenters the original vessel diameter, the fluid layers separate, allowing formation of stagnation zones and regions of recirculation. 


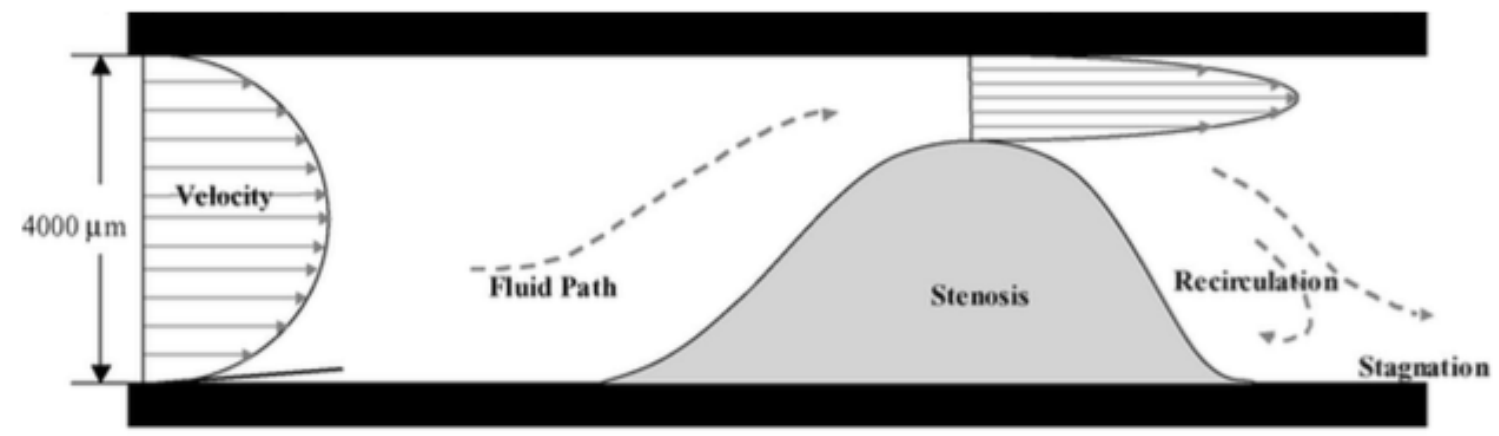

Figure 3. Velocity field of blood flow within an artery

As the fluid exits the area of stenosis, the streamlines do not follow the contour of the vessel, causing flow separation. This can result in regions of low shear stress near the vessel wall, and increase the time that the fluid resides near a specific area. In the areas of stagnation and recirculation, the fluid shearing forces are not great enough to overcome cell-cell interaction forces. Interactions between leukocytes, erythrocytes, and platelets occur at low shear stresses, and are known to increase blood viscosity [22]. The viscosity of blood depends on shear rate. Blood viscosity is affected by changes to shear rate at low levels of hematocrit. However, as the level of hematocrit increases, the shear rate effect on the change in blood viscosity becomes greater. In areas of high shear rate $\left(\sim 300 \mathrm{~s}^{-1}\right)$, for example the aorta, blood viscosity is approximately $3.5 \mathrm{cP}$, while in vessels with lower shear rates $\left(\sim 5 \mathrm{~s}^{-1}\right)$, as in veins, the blood viscosity is higher $(\sim 10 \mathrm{cP})$ [24].

The velocity of the fluid is zero at the vessel wall, and reaches a maximum at the center of the vessel. Assuming the blood vessel is a straight, cylindrical tube with rigid walls, the shear rate can be found using Equation 3.

Equation 3:

$$
\dot{\gamma}_{-r z}=\frac{8 u}{d}
$$


Here, $\mathrm{u}$ is the average velocity, $\mathrm{Q}$ is the mean volumetric flow, and $\mathrm{d}$ is the vessel diameter [24].

For blood flow within a vessel, the shear stress is defined by the Hagen-Poisseuille equation, where $\mathrm{Q}$ is the mean volumetric flow rate, $\mathrm{u}$ is the mean velocity, and $\mathrm{d}$ is the vessel diameter. Equation 4 states that shear stress is directly proportional to blood velocity, and inversely proportional to vessel diameter [24]. Equation 5 is a variation of Equation 4, stating that shear stress is directly proportional to flow rate, and inversely proportional to vessel diameter.

Equation 4:

$$
\tau_{-r z}=8 \mu \frac{u}{d}
$$

Equation 5: $\quad \tau_{-r z}=32 \mu \frac{Q}{\pi * d^{3}}$

The Haagen-Poisseuille equation is applied under four assumptions. First, the blood is considered a Newtonian fluid. Second, the vessel cross sectional area is cylindrical. Third, the vessel is straight with inelastic walls. Fourth, the flow is steady and laminar [24].

Normal shear stresses at the walls of an artery have been demonstrated to exist between $10-70$ dynes $/ \mathrm{cm}^{2}$ [24]. Shear stresses in this range stabilize blood vessels and promote healthy remodeling following an injury [23]. Higher values have been detected in regions 
of vasculature with turbulent flow, or increased flow velocity, including arteries with extreme curvature (i.e aortic arch and bifurcations) (Figure 4) [23]. Low shear stress causes the vessel diameter to decrease, and leads to a mean shear stress level around 15 dynes $/ \mathrm{cm}^{2}$. Low shear stress is typically in regions of unstable blood flow, including those of recirculation and stagnation [24].

High shear stress can be found in laminar flow, and is known to promote endothelial cell survival and quiescence, alignment in the direction of flow, and secretion of substances

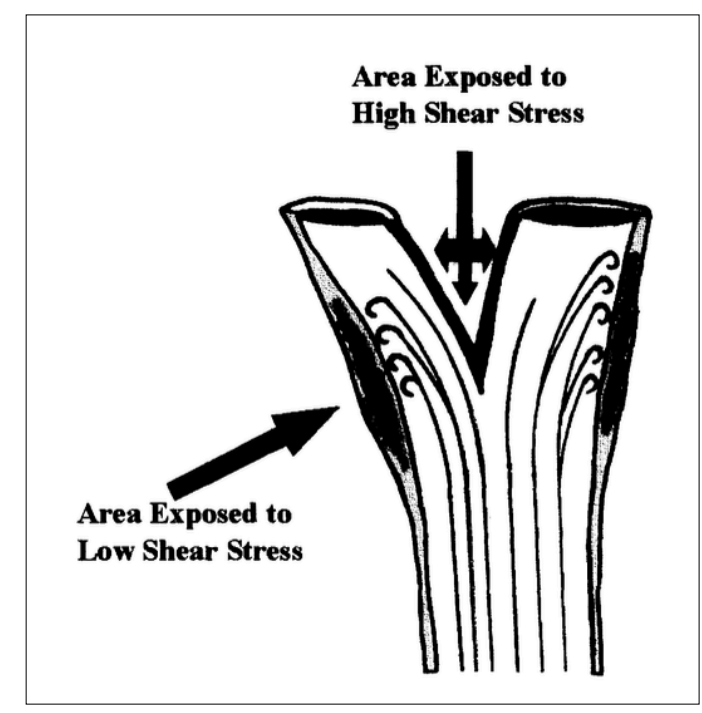

Figure 4. Bifurcation in an artery and the resulting regions of high shear stress and low shear stress.

promoting vasodilation and anticoagulation. Low shear stresses or reversing shear stresses, as those found in turbulent flow, promote endothelial proliferation and apoptosis, shape change, and secretion of substances that promote vasoconstriction, coagulation, and platelet aggregation [23]. Long-term changes in the signaling and structure of the vessel through shear stress can alter regulation of protein synthesis and gene expression, which can lead to changes in the vessel wall. These changes can include the proliferation and migration of SMCs and expression of endothelial cell-surface 
molecules that stimulate leukocyte adhesion and migration [23]. These changes can cause a weakening of the vessel wall, agitating the effects of an aneurysm.

One method to determine the velocity gradient is to consider the change in linear velocity between two points on the vessel's diameter. The shear rate can be determined from Equation 6:

Equation 6: $\quad \dot{\gamma}_{-r z}=\frac{\Delta u}{\Delta r}$

However, shear rates calculated using this equation are overestimated by anywhere between $10-45 \%$. A potential cause for this is the inability for accurate arterial wall tracking [24]. While these estimates may not be perfect, they give an idea of the shear rate within the artery. It is important to determine the shear rate and shear stresses inside the artery to understand how the aneurysm is progressing.

\subsubsection{Shear Stress Physiology}

It is known that turbulent flow leads to complex changes in shear stress magnitude and direction. There are a number of factors and forces that affect the endothelial cell. Chemical forces may be autocrine, paracrine, or endocrine. Physical forces can be from pressure, strain, or shear stress. (Figure 5). The magnitude of shear stress on the 


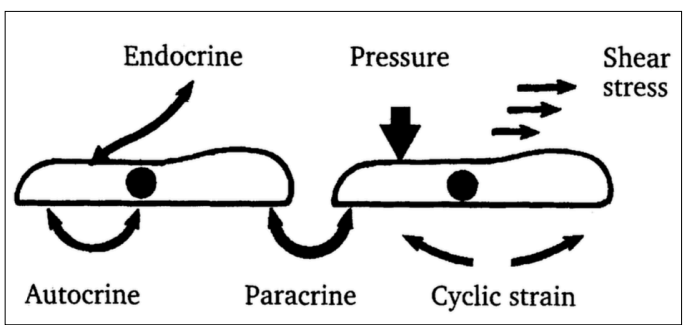

Figure 5. Chemical forces acting on endothelial cells

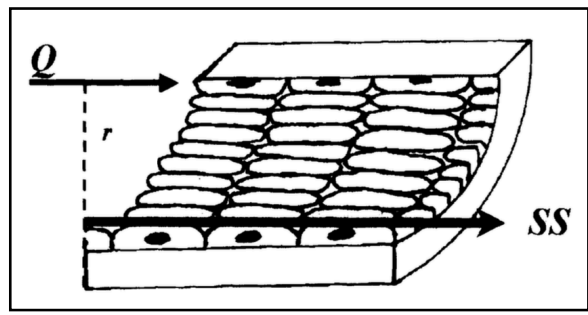

Figure 6. Shear stress acting on endothelial cells

endothelium (SS) is proportional to Q (volumetric flow rate) of fluid flow in the vessel, inversely proportional to $r$ (the radius of the blood vessel) (Figure 6) [23].

Shear stress values within a normal physiological range can stimulate endothelial cells to release factors that regulate the coagulation pathway. Coagulation plays a role in the growth of aneurysms. These factors include prostacyclin, nitric oxide (NO), calcium, thrombomodulin, tissue plasminogen activator (tPA), and plasminogen activator inhibitor type-I (PAI-1). Normal shear stress stimulates endothelial cells to release antithrombotic

\begin{tabular}{|c|c|c|c|}
\hline \multirow{3}{*}{$\begin{array}{l}\text { Laminar blood flow: } \\
\text { high shear stress }\end{array}$} & Quiescent phenotype & & \\
\hline & Coagulation & Endothelium & Vessel wall \\
\hline & $\begin{array}{l}\text { I } \mathrm{PGI}_{1}, \mathrm{NO} \text {, } \mathrm{tPA} \\
\text { I Platelet aggregation }\end{array}$ & $\begin{array}{l}\text { 1 Proliferation } \\
\text { 1 Apoptosis } \\
\text { † Alignment }\end{array}$ & $\begin{array}{l}\text { I SMC proliferation } \\
\text { \ Leukocyte adhesion } \\
\text { quiescent }\end{array}$ \\
\hline \multirow{3}{*}{$\begin{array}{l}\text { Tubulent blood flow: } \\
\text { low shear stress }\end{array}$} & Activated phenotype & & \\
\hline & Coagulation & Endothelium & Vessel wall \\
\hline & $\begin{array}{l}\text { I } \mathrm{PGI}_{1}, \mathrm{NO} \text {, tPA } \\
\text { I Platelet aggregation }\end{array}$ & $\begin{array}{l}\text { | Proliferation } \\
\text { | Apoptosis } \\
\text { | Alignment }\end{array}$ & $\begin{array}{l}\text { I SMC proliferation } \\
\text { I Leukocyte adhesion } \\
\text { Atherogenic }\end{array}$ \\
\hline
\end{tabular}

Figure 7. Effect of high and low shear stress on the signaling factors of the endothelium

agents, including prostacyclin, nitric oxide (NO), calcium, and thrombomodulin.

Thrombomodulin is the major anticoagulant cofactor, and is up-regulated by high shear stress [19]. 
Shear stress can affect the binding of leukocytes. Leukocyte binding and chemoattractant expression of endothelial cells are inhibited at high levels of shear stress, while low shear stresses stimulate leukocyte binding. The phenotype of endothelial cells is dependent on the hemodynamic environment; high shear stress environments encourage quiescent cells, activated cells are found in environments of low shear stress (Figure 7) [23].

Blood vessels are under constant mechanical loading, coming from blood pressure and flow, which causes endothelial shear stress and circumferential wall stress, respectively [19]. Shear stress plays a role in vasodilation. Nitric oxide (NO) generated from endothelial NO synthase (eNOS) mediates the response to shear stress [19]. Elevated blood pressure leads to increased pressure on the vessel wall, which induces vascular remodeling. This remodeling leads to increased wall thickness, which results in increased small vessel resistance, and increased large vessel compliance. [19].

The magnitude and direction of blood flow, as well as the activity of nicotinamide adenine dinucleotide phosphate (NADPH) oxidase affect flow-mediated dilation of the vessels. Shear stress caused by the blood flow activates a number of different pathways through the endothelial surface molecules, including platelet endothelial cell adhesion molecule (PECAM-1), integrins, ion channels, and tyrosine kinase receptors. Shear stress results in inactivation of vascular NADPH oxidase and reduced superoxide production. Tension and deformation of the endothelium can also activate integrins, which are important for shear-induced vasodilation. Shear stress-induced endothelium integrin 
activation can further stimulate phosphorylation of eNOS. This results in activated eNOS and increased NO production, leading to further vasodilation of the artery [19].

\subsubsection{Low Shear Stress}

At low shear conditions, plasma proteins allow for the formation of cell-cell bridges, which change the motion and velocity of the cells. Rouleaux formation is one of the bestknown examples in which erythrocytes aggregate, resembling a 2-dimensional stacking of coins. However, erythrocytes may combine to form more complex 3D clumps, increasing the local blood viscosity. The increased viscosity results in decreased blood flow, and therefore, reduced shear rates [22]. The apparent viscosity of blood increases in a non-Newtonian manner as the shear rates decrease below $100 \mathrm{~s}^{-1}$. These low shear conditions can promote other cell-cell interactions involving platelets, leukocytes, and red cells. Goel and Diamond demonstrated that at shear rates below $100 \mathrm{~s}^{-1}$, there exist receptor-ligand interactions that regulate the association of red cells with platelets and neutrophils. Flow resistance can be further increased during inflammation by leukocyte adhesion to the vessel wall, as well as leukocyte-red cell interactions [22]. Low shear stress can contribute to the initiation of atherosclerosis, particularly at the vessel wall. Low shear stress contributes to a high inflammatory and a high prothrombotic state [19].

Under normal conditions, healthy endothelium exposed to physiological shear stresses serves as a nonthrombogenic, atheroprotective surface. It regulates functions including vasomotor tone, as well as long-term vessel remodeling through responding to local 
physical forces. The endothelial cells align and elongate in the direction of flow, and stimulate increased levels of cyclooxygenase-2, prostaglandin $\mathrm{I}_{2}$, Nitric Oxide (NO), and antioxidants. In oscillatory or reversed shear stress regions, such as those at bifurcations in a vessel, low arterial wall shear stresses $\left(<4 \mathrm{dyne} / \mathrm{cm}^{2}\right)$ are associated with expression of nuclear factor B-regulated genes. Expression of these genes leads to inflammation, as well as leukocyte recruitment and extravasation [22].

\subsubsection{High Shear Stress}

Rupture of an AAA occurs when the mechanical stress on the walls exceeds the strength of the wall tissue. It is important to understand where the regions of high shear stress in the vessel are located in order to help prevent this rupture. Raghavan et al conducted a study to determine the peak wall stress, as well as the failure strength, on aneurysmal and nonaneurysmal aortas. The group found that AAA volume, not diameter, was a better indicator of high wall stresses, and could potentially help predict AAA rupture [25]. Figure 8 shows the correlation coefficient of the AAA diameter, height, volume, and systolic pressure. The group concluded that AAA volume has the greatest effect on peak wall shear stress. The peak wall stress for a nonaneurysmal aorta was found to be 9 $\mathrm{N} / \mathrm{cm}^{2}$. The peak wall stress for a 4-cm AAA and an 8-cm AAA was found to be 23 $\mathrm{N} / \mathrm{cm}^{2}$ and $45 \mathrm{~N} / \mathrm{cm}^{2}$, respectively. Mechanical testing of healthy and aneurysmal abdominal aortic wall samples showed a failure strength of the nonaneurysmal aorta of $121 \mathrm{~N} / \mathrm{cm}^{2}$, while the failure strength of an aneurysmal aorta was $65 \mathrm{~N} / \mathrm{cm}^{2}$. AAA rupture 
may occur during the growth of the aneurysm, when the increasing wall stress exceeds the decreasing failure strength of the aortic tissue [25].

\begin{tabular}{|lc|}
\hline Clinical factor & $\begin{array}{c}\text { Correlation } \\
\text { coefficient with } \\
\text { peak } \text { wall stress* }\end{array}$ \\
\hline AAA diameter & 0.56 \\
AAA height & 0.57 \\
AAA volume & 0.70 \\
Systolic pressure & 0.55 \\
\hline *Excluding the control. & \\
$A A A$, Abdominal aortic aneurysm. & \\
\hline
\end{tabular}

Figure 8. The correlation coefficients of clinically measurable factors with peak wall stress on the 6 AAAs.

Peak wall stress on the AAAs studied was anywhere between $45 \%$ to $69 \%$ of their failure strength. Peak wall stress of the nonaneurysmal aorta was less than $10 \%$ of its failure strength. The location of the peak wall stress in all AAAs was the posterior surface. The site of maximum stress is also likely to be the site of rupture. Darling et al examined autopsies of 118 patients with ruptured AAAs. In $82 \%$ of all cases, the rupture was located on the posterior surface [25].

\subsubsection{Reynolds Number and the Effect on Blood Flow}

The Reynolds number is a dimensionless quantity used to determine the transition between steady flow and turbulent flow. It is the ratio between the inertial forces and viscous forces within the liquid. This relationship can be seen in Equation 7 
Equation 7: $\quad R e=\frac{\rho v D}{\mu}$

In this equation, $\rho$ is the density of the fluid, $v$ is the average fluid velocity, $\mathrm{D}$ is the inner diameter of the pipe through which the fluid is flowing, and $\mu$ is the viscosity of the fluid.

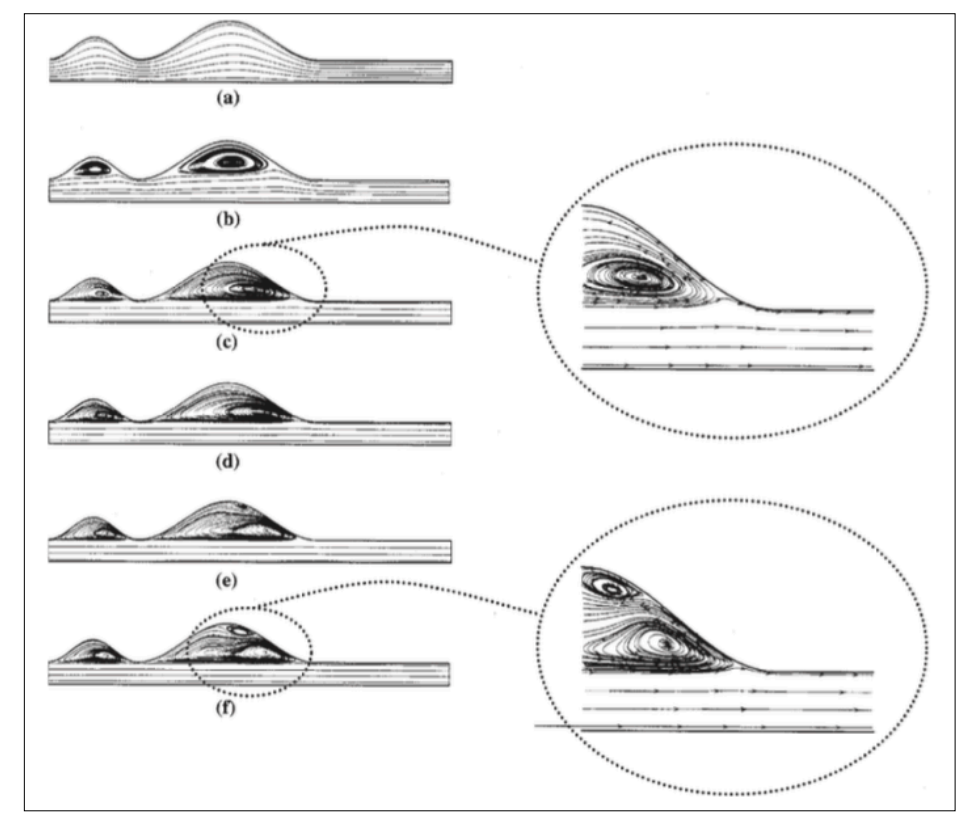

Figure 9. Streamlines for laminar steady flow at (a) $\operatorname{Re}=10$; (b) $\operatorname{Re}=100 ;$ (c) $\operatorname{Re}=500 ;(d) \operatorname{Re}=1000 ;(e) \operatorname{Re}=1750$; and (f) $\operatorname{Re}=$ 2265: The direction of the flow is from left to right $[3 \mathrm{P}]$.

For fully developed flow, a Reynolds number below 2000 designates laminar flow. A Reynolds number above 4000 signifies turbulent flow. A Reynolds number between 2000 and 4000 describes transitional flow. 
Figure 9 shows the laminar flow streamline associated with various Reynolds number values within the range of $10 \leq \operatorname{Re} \leq 2265$. At $\operatorname{Re}=10$, given a blood flow rate of 0.12 $\mathrm{L} / \mathrm{min}$, there is no flow separation, and the main stream of fluid fills the arterial distensions. Flow separation begins at a Reynolds number in the range of $\mathrm{Re}=25$. Figure 9 (b) through (f) show inner streamlines that are unaffected by the dilations, as well as regions of flow recirculation and stagnation. At Reynolds numbers above 500, the reverse flow occupies most of the volume of the aneurysm sac. The upper region of the aneurysm sac is slowly moving fluid particles that are basically stagnant. These have high distal and low proximal shear values. Finol et al observed that as the Reynolds number increased, the center of the recirculating flow regions moves downstream, as well as closer to the main stream. This displaced the boundary layer separation points further upstream, and

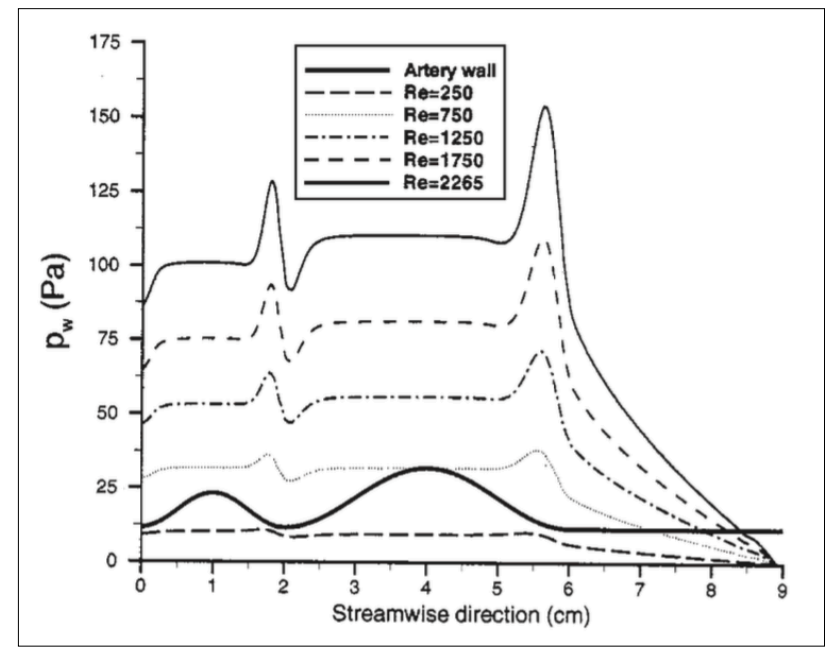

Figure 10. Pressure distribution along the wall of the double-aneurysm model for selected Reynolds numbers.

the reattachment points further downstream. This displacement leads to an increased volume occupied by the stagnation zones within the aneurysm sacs [3]. 
Figure 10 shows the distribution of wall pressure throughout the aneurysm model, relative to the exit pressure of $0 \mathrm{~Pa}$ [3]. At this stagnation zone, the wall pressure peaks, then decreases at the distal end of the aneurysm [3]. This peak in pressure applies stress to the artery wall, playing a role in the dilation of the artery and promoting aneurysm

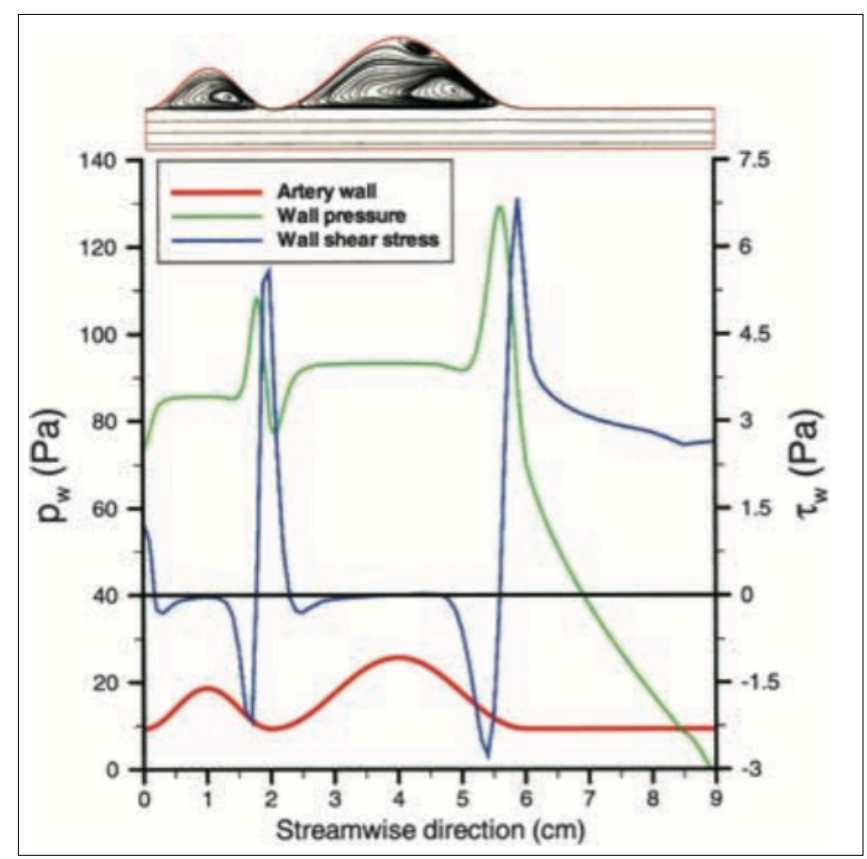

Figure 11. Wall pressure, wall shear stress, and streamlines for $\operatorname{Re}=\mathbf{2 0 0 0}$

formation. Figure 11 displays the relationship between wall pressure and shear stress at $\mathrm{Re}=2000$ [3]. It can be seen that in the stagnation zones, there is a relationship between the wall shear stress and the wall pressure. When the pressure increases, the shear stress also increases. These factors create an environment in which the artery wall dilates, and the aneurysm can progress. 


\subsection{Dimensions}

The abdominal aorta is the large vessel from the heart that travels through the middle of the abdomen and bifurcates into two arteries supplying the legs with blood [1]. The human aorta measures $75 \mathrm{~cm}$ from the aortic valve to the iliac bifurcation. Along this path, the aorta distributes oxygenated blood to all other minor arteries, apart from those of the lungs. The aorta supplies the heart through coronary arteries, the brain through supra-aortic vessels, and the organs of the abdomen through the abdominal aorta. The normal diameter of the infrarenal aorta in a population over 50 years old is $1.5 \mathrm{~cm}$ in women and $1.7 \mathrm{~cm}$ in men. AAAs are defined as an aortic diameter at $150 \%$ of the normal diameter at the level of the renal arteries [2]. An infrarenal aorta that is over $3 \mathrm{~cm}$ in diameter is considered an aneurysm, even if asymptomatic [20].

One of the strongest predictors of the risk of aneurysm rupture is the aneurysm size. This risk greatly increases once the diameter reaches $5.5 \mathrm{~cm}$. The Joint Council of the American Association for Vascular Surgery and Society for Vascular Surgery estimated the annual rupture risk based on AAA diameter as the following [2]:

1. Less than $4.0 \mathrm{~cm}$ in diameter $-0 \%$

2. $4.0 \mathrm{~cm}$ to $4.9 \mathrm{~cm}$ in diameter $-0.5 \%$ to $5 \%$

3. $5.0 \mathrm{~cm}$ to $5.9 \mathrm{~cm}$ in diameter $-3 \%$ to $15 \%$

4. $6.0 \mathrm{~cm}$ to $6.9 \mathrm{~cm}$ in diameter $-10 \%$ to $20 \%$

5. $7.0 \mathrm{~cm}$ to $7.9 \mathrm{~cm}$ in diameter $-20 \%$ to $40 \%$

6. $8.0 \mathrm{~cm}$ in diameter or greater $-30 \%$ to $50 \%$ 


\subsection{Characteristics of Blood}

Blood is an assortment of cells, proteins, lipoproteins, and ions. This mixture is responsible for transporting nutrients and waste throughout the body. Red blood cells (RBCs) account for roughly $40 \%$ of blood by volume [2]. RBCs affect the viscosity of the blood as well as its behavior. The viscosity of blood is not constant at all flow rates,

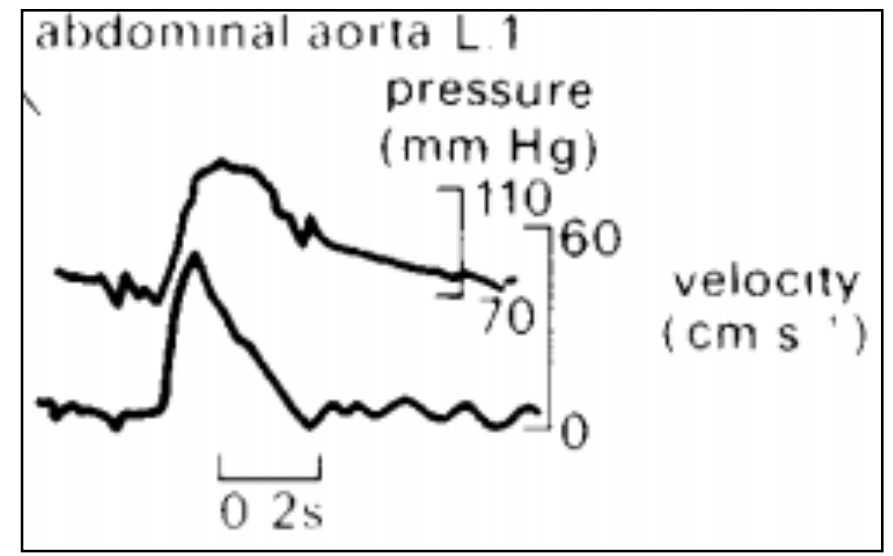

Figure 12: Pressure and velocity pulse waveforms in the aorta and arterial branches of a dog. The pressure maximum becomes amplified while the velocity maximum decreases as the blood moves downstream.

and can display non-Newtonian behavior at various shear rates. This non-Newtonian behavior is most clearly observed at low shear rates as the RBCs clump together. Blood is ejected when the heart contracts during systole, and does not pump when the heart rests during diastole. The role of the aorta is that of a compliance chamber providing a reservoir of high pressure during diastole and systole. Pressure and flow have a pulsatile pattern, as shown in Figure 12 [1]. 


\subsection{Pathophysiology and Etiology}

The US National Heart, Lung, and Blood Institute identified a number of mechanisms through which AAAs arise including proteolytic degradation of aortic wall connective tissue, inflammation and immune responses, biomechanical wall stress, and molecular genetics [20]. AAAs occur as a result of a breakdown of the structural proteins that support the aorta, including elastin and collagen. The cause is unknown, although genetic predisposition is a factor. AAAs arise as a result of deterioration of the media, although all layers of the tissue are distended. This distension leads to widening of the vessel lumen and loss of structural integrity [20].

Approximately $90 \%$ of AAAs are infrarenal, which could be a result of a number of factors. There is a decrease in the number of medial elastin layers from the proximal thoracic aorta to the infrarenal aorta, along with thinning of the media and thickening of the intima [20]. Thinning of the media results in reduced levels of elastin and collagen. This decrease in the number of medial elastin layers could be due to the decrease of the diameter of the aorta from the thoracic region to the abdominal and infrarenal regions [7].

\subsection{Symptoms}

Abdominal aneurysms are particularly dangerous because they can often progress without symptoms. However, in more severe aneurysm cases, some of the symptoms that may be present include a throbbing feeling in the abdomen, and pain in the back or the side of your abdomen that can last for days. If the AAA ruptures, symptoms can include sudden 
pain in the lower abdomen and back, nausea and vomiting, constipation and problems with urination, clammy or sweaty skin, light-headedness, and an elevated heart rate when standing up [26].

\subsection{Diagnosis}

A healthcare provider can examine the abdomen, and feel the pulses in the legs. Based on these tests, the provider may find a lump in the abdomen, a pulsating sensation in the abdomen, a second or echoed heartbeat in the abdomen, or a stiff or rigid abdomen [27].

For more definitive results, a number of diagnostic procedures are used to diagnose the AAA. The first is ultrasound and echocardiography. These tests use sound waves to create images of the structures inside the body, and can show the size of the aortic aneurysm [28]. A Computed Tomography (CT) scan uses x-rays to create clear images of organs. Dye is injected into the arm, making the arteries visible on the CT scan pictures. This test can show the size and shape of the aneurysm. These pictures are more detailed than those using ultrasound or echocardiography [28]. Magnetic Resonance Imaging (MRI) uses magnets and radio waves to create images of the organs and structures of the body. MRI can precisely detect the size and exact location of the aneurysm [28].

Angiography uses a dye and x-rays to show the insides of the arteries. The function of this test is to display the amount of damage and blockage in the blood vessels. Angiography shows the location and size of the aneurysm [28]. 


\subsection{Treatment}

Endovascular aneurysm repair (EVAR) is used to help repair and reverse the aneurysm. The two most common treatments for the AAA are interventional repair and surgical repair. Interventional repair is a minimally invasive procedure using imaging techniques. An incision is made in the groin, into which a catheter containing a compressed stent graft is passed into the femoral artery. The catheter is then guided to the AAA, and opened when in the proper position, creating new walls through which the blood flows [29]. There are advantages and disadvantages to this interventional repair. The advantages include no abdominal surgical incision, minimal sutures, faster recovery time, no general anesthesia in some cases, less pain, and reduced complications [29]. The disadvantages include possible migration of the graft following treatment, allowing blood flow into the aneurysm, and a life-time requirement for follow-up studies to verify that the stent graft is continuing to function [29]. Surgical repair is the most common treatment for large, unruptured aneurysms. This procedure involves an incision from just below the breastbone to the top of the pubic bone. The aorta is clamped off, and the aneurysm is then cut open. A graft is sewn in place to act as a bridge for the blood flow. The blood then flows through the graft, and no longer allows the pressure from the blood to further distend the aorta wall [29].

Fatality associated with elective surgical repair was found to be $\sim 5 \%$ in one study [21]. The population-based study from the Mayo clinic reported a 5-year survival of $60 \%$ after the repair of AAAs with a diameter greater than $5 \mathrm{~cm}$. A study in Berkshire, showed that 
the 5-year survival rate of patients who underwent surveillance of AAAs less than $4 \mathrm{~cm}$ and $4.5-5.0 \mathrm{~cm}$ in diameter was $62 \%$ and $45 \%$, respectively [21].

\subsection{General Approach}

In this thesis, I will discuss my approach to creating a model of the AAA, and my results from the study. In Section 1, I discuss the goals for this project. In Section 2, I will give background information about aneurysm formation relating to shear stress and blood flow, as well as current methods for detection and treatment of aneurysms. In Section 3, I will discuss the manufacturing approach for making the vascular construct. Section 4 shows the experimental set up for the flow visualization experiments. Section 5 discusses the results of the flow visualization experiments, as well as the relevant statistical analysis. In Section 6, the developed protocol is discussed, as well as future iterations of this project. 


\section{CHAPTER 3: MATERIALS AND METHODS}

\subsection{CAD}

The Computer Aided Design (CAD) model was created following the directions in Appendix A. The Digital Imaging and Communications in Medicine (DICOM) file used to create the aneurysm model was found online on the OsiriX website [30]. OsiriX is a software used to view and manipulate DICOM images. The file chosen was titled "Panaoramix". This file was an abdominal CT angiogram acquired on a 16-detector scanner in a patient with an AAA. The DICOM image was imported into OsiriX Lite. The threshold was adjusted so that the aneurysm had the greatest visibility. A segmentation was created for bone, so it could be selected and removed from the image. A segmentation was created for the blood vessels. The remaining information in the file is all the blood vessels branching from the abdominal aorta. All extraneous vessels along the abdominal aorta were removed, leaving only the abdominal aorta, the renal arteries, and the bifurcation of the aorta towards the iliac arteries. The file was then saved as a stereolithography (STL) file to be manipulated in Autodesk Meshmixer.

Autodesk Meshmixer is software used to edit STL files. The protocol for editing the aneurysm model can be found in Appendix B. The STL file edited in OsiriX Lite was imported into Autodesk Meshmixer. The object was made solid, and the edges were smoothed. Unnecessary edges and bumps were removed from the model, and the edges were smoothed again. This removed overlapping faces. The aneurysm model was then saved as an STL file. 
The STL was exported from Meshmixer, then imported into Solidworks, following Appendix C. The STL file was imported into Solidworks as a solid body. Import Diagnostics were run on the part to detect any faulty faces or gaps in the geometry. A new reference plane was created parallel to the front plane above the top of the neck of the aneurysm. A rectangle was sketched, and used to make an extruded cut. The aneurysm model was cut just below the renal arteries to allow for the flow visualization trials to focus on the sac of the aneurysm. The process of creating a new reference plane, and making an extruded cut was repeated for both of the legs of the aneurysm to create a flat face for use as outlets for the COMSOL Multiphysics ${ }^{\circledR}$ model. The file was saved again as a STL file.

Meshlab was used to reduce the number of faces in the STL file to allow for quicker calculations in COMSOL Multiphysics ${ }^{\circledR}$. A detailed description of this procedure can be found in Appendix D. The STL file was imported into Meshlab. Quadratic Edge Collapse Decimation was used to reduce the number of faces in the aneurysm model. The model was reduced by $50 \%$, where the geometry of the aneurysm remained intact, but unnecessary faces were removed. The file was then saved as a STL file.

The aneurysm model was scaled to the correct dimensions in Solidworks. A detailed description of this process can be found in Appendix E. The STL file was imported into Solidworks as a solid body. Import Diagnostics were run on the model to detect any faulty faces or gaps in the geometry. The diameter of the neck of the aneurysm was measured using the Measure tool. The length was 18.17 centimeters, which is $10 x$ larger 
than it should be. The model was scaled by a factor of $1 / 10$ to create a model with the correct dimensions. The file was then saved as an IGES file to be imported into COMSOL Multiphysics $\circledR_{\text {. }}$

\subsection{Full Vessel Construct}

\subsubsection{D Printing}

Fused deposition modeling (FDM) is a manufacturing process that allows for accurate and rapid modeling of complex parts, which would otherwise require a complicated manufacturing process. FDM involves taking a computer file, and using a 3D printer to

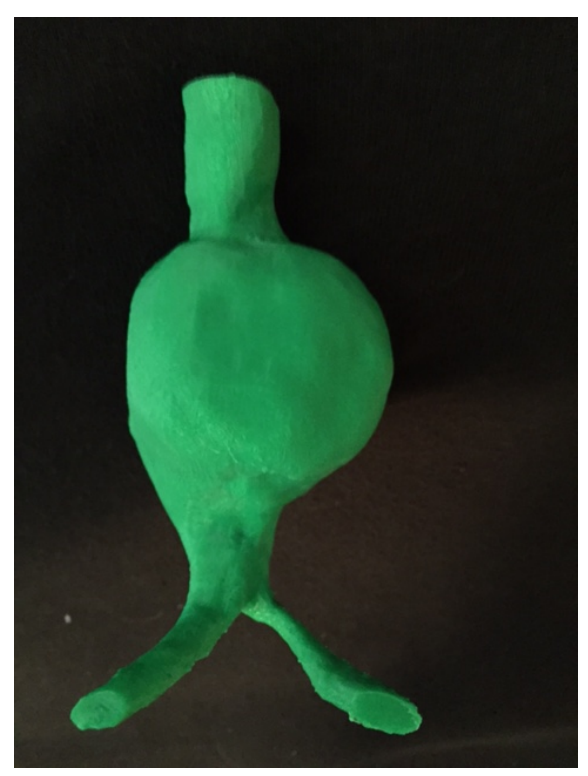

Figure 13. 3D print of the abdominal aortic aneurysm using PLA.

print the object using the desired material. The aneurysm file was converted from a DICOM file to a STL file, then processed using a slicer program (Cura) that converts the model into instructions for the 3D printer (G-code). The code sliced the model into very 
thin layers in the $\mathrm{x}-\mathrm{y}$ orientation. Polylactic acid (PLA) was heated and extruded from the nozzle of the printer along the $\mathrm{x}-\mathrm{y}$ axis in a crisscrossing pattern. Once one level was completed, the printer raised one increment in the $\mathrm{z}$-direction, and continued to print the model along that level. This process was continued until the aneurysm was complete. Support material was required for any overhangs, however this material was removed from the model after the manufacturing was finished. The build volume for the Printrbot Simple was 6" x 6" x 6", with a print speed of $80 \mathrm{~mm} / \mathrm{sec}$, and a print resolution of 50 microns [31]. The printed model of the AAA can be seen in Figure 13.

\subsubsection{Mold}

The manufacturing of the mold for the AAA was based on the method of lost wax casting. This process begins with an object that is to be replicated; in this case we have a PLA model of the AAA. A mold is made from the object, and the object is removed from the mold, leaving a hollow chamber in the mold with the same geometries as the original object. Wax is then poured into this hollow chamber, giving a replica of the original object. This wax model is then submerged in another material, in our case, Polydimethylsiloxane (PDMS). The wax is melted from the PDMS, and the final result is a clear chamber with a hollow region that has the same dimensions and geometries as the original PLA aneurysm model. 
PDMS was used as the material to create the aneurysm mold. PDMS was chosen because it is a malleable plastic that is transparent, allowing for easy flow visualization. QUICKSIL RTV Jewelry Molding Rubber was used to create the mold of the AAA. Part A and Part B were mixed at a 1:1 volume ratio, and spread to encase the $3 \mathrm{D}$ printed AAA seen in Figure 13. Only the entryway to the aneurysm through the aorta was left open. The

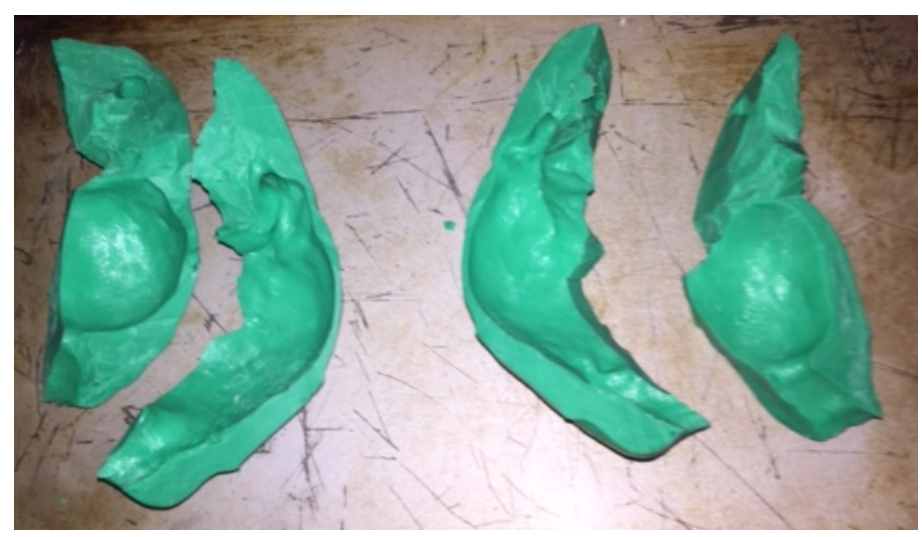

Figure 14. The molding rubber was cut from the 3D printed aneurysm using an $\mathrm{X}$-Acto knife.

molding rubber was allowed to cure for an hour. Once cured, the material was then cut from the aneurysm into quarters using an X-Acto knife. Notches were made when cutting the rubber from the PLA aneurysm, so that the rubber mold could be assembled without the 3D printed aneurysm inside (Figure 14). The PLA aneurysm was removed from the rubber mold, and the mold was placed back together in one piece. The individual pieces were held together using rubber bands (Figure 15). Paraffin wax was placed in a container above boiling water. Once the wax was liquid, a funnel was placed above the opening to the rubber mold. The paraffin wax was poured into the aneurysm mold, and then allowed to cool in a freezer. A wooden stick was placed into the top of the wax mold 
and was secured in place as the wax hardened. Once the wax had hardened, the mold was removed from the freezer, and the rubber bands were removed from the mold. The four pieces of the rubber mold were peeled from the wax model, taking extreme care around the legs of the aneurysm, as these were the thinnest and most fragile parts of the mold.

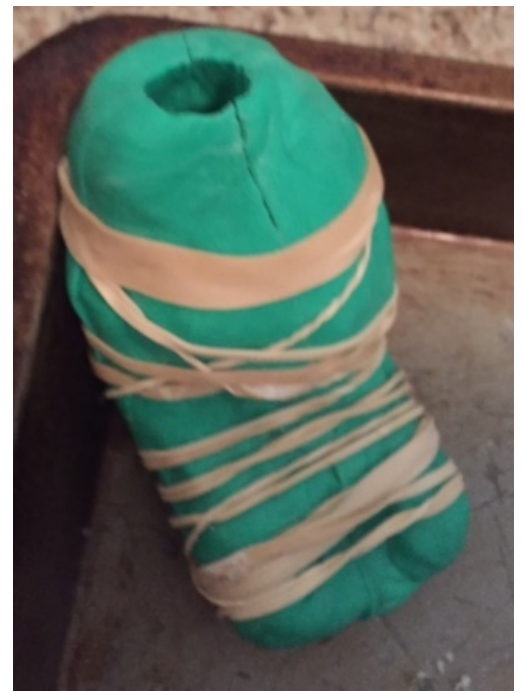

Figure 15. Rubber aneurysm mold with a hollow chamber into which liquid paraffin wax was poured.

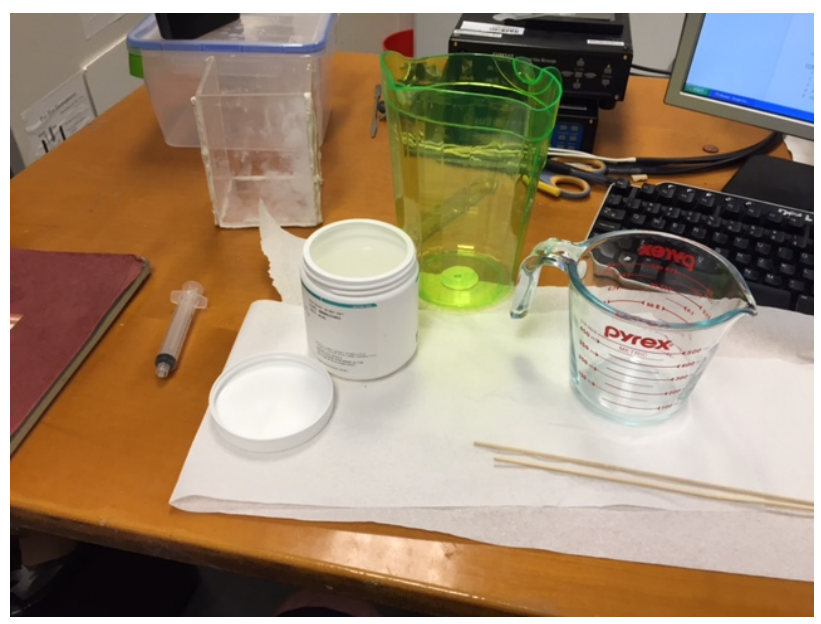

Figure 16. The acrylic box and the ingredients used to create

Acrylic plastic was used to manufacture the chamber into which the PDMS was poured. The dimensions of the aneurysm model were $9.8 \mathrm{~cm}$ in height, $6.3 \mathrm{~cm}$ in width, and 6.6 $\mathrm{cm}$ in length. The chamber dimensions were therefore made to be $14 \mathrm{~cm}$ in height, $10 \mathrm{~cm}$ in width, and $10 \mathrm{~cm}$ in length. The acrylic plastic was cut using a bandsaw. Caulk was used to seal the corner, ensuring there would be no leaks when the PDMS was poured.

The dimensions of the acrylic box were $10 \mathrm{~cm} \times 10 \mathrm{~cm} \times 14 \mathrm{~cm}$, and the thickness of the acrylic was $0.5 \mathrm{~cm}$. The available space of the box was $9.5 \mathrm{~cm}$ x $10 \mathrm{~cm}$ x $13.5 \mathrm{~cm}$. From these dimensions, the volume of the box was $1282.4 \mathrm{~cm}^{3}$. The volume of the wax aneurysm model was $100 \mathrm{~mL}$. The volume of the PDMS to be used to make the aneurysm 
mold was $1182.5 \mathrm{~mL}$. The PDMS consists of two parts, the elastomer base (part A), and the elastomer curing agent (part B). The mixing ratio of the two parts is $10(\mathrm{~A})$ : $1(\mathrm{~B})$. Rounding up the volume of PDMS used to $1200 \mathrm{ml}$, gives $1100 \mathrm{~mL}$ of part A and $110 \mathrm{~mL}$

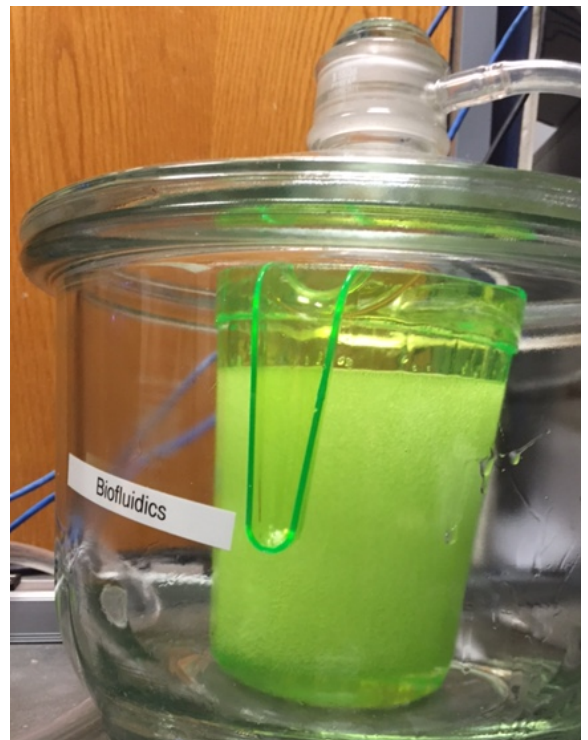

Figure 17. Part $A$ and Part $B$ mixed and in the process of vacuum degassing

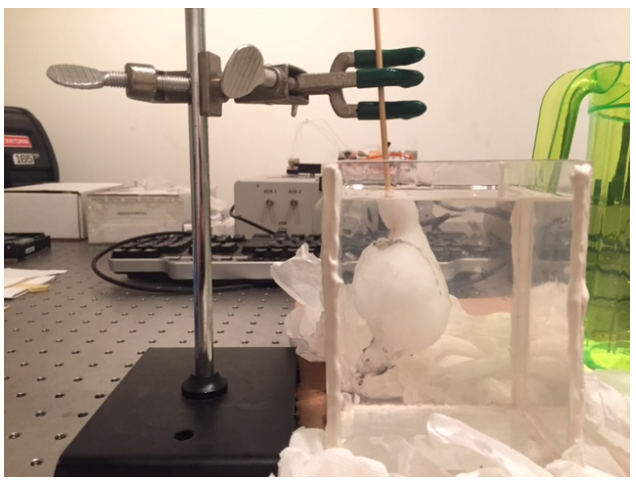

Figure 18. The Wax model suspended in the acrylic box

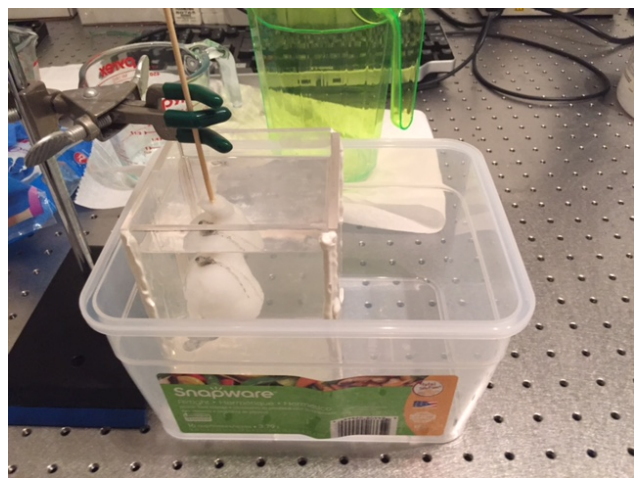

Figure 19. The Wax model suspended in the acrylic box

of part B. The acrylic box and items used to create the PDMS mold can be seen in Figure

16. Part A and B were measured into the same container and mixed thoroughly for five minutes. The container was then placed in a vacuum degasser for two hours to get rid of the bubbles that appeared from mixing the two parts (Figure 17). The wax model was suspended above the acrylic box using the wooden skewer (Figure 18 and 19). The 
PDMS was poured into the acrylic box until it reached the top of the aneurysm. The edges of the acrylic box had not been properly sealed, a portion of the PDMS leaked out. However, the aneurysm sac was unaffected. The PDMS was allowed to cure for 72 hours. When the PDMS was completely cured, the mold was removed from the acrylic box. The aneurysm model can be seen in Figure 20 and 21.

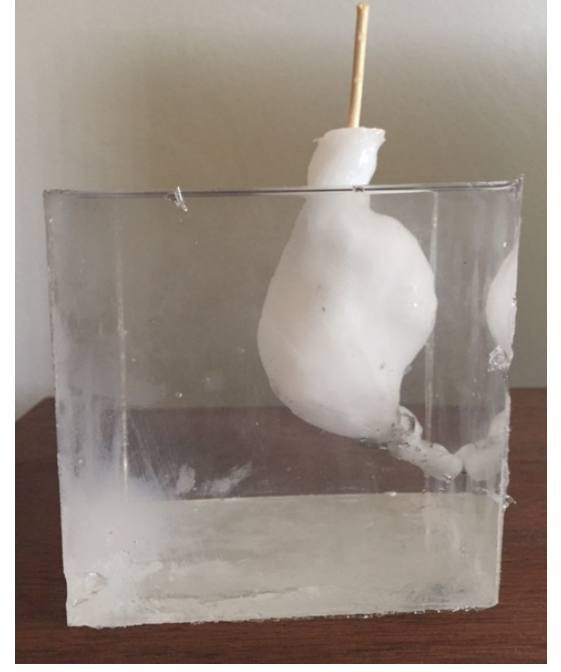

Figure 20. Side view of the wax inside the PDMS mold

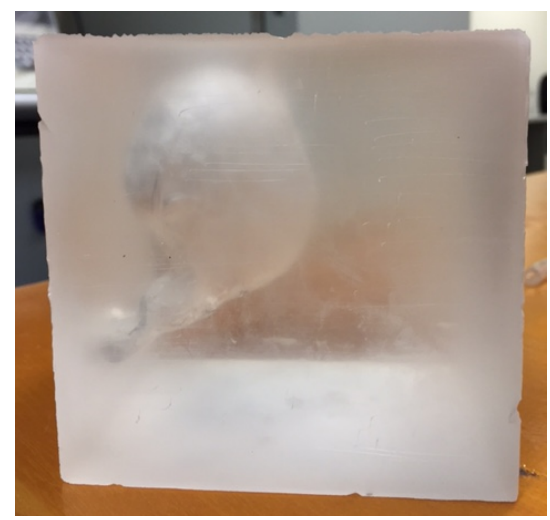

Figure 22. Side view of the hollow PDMS mold

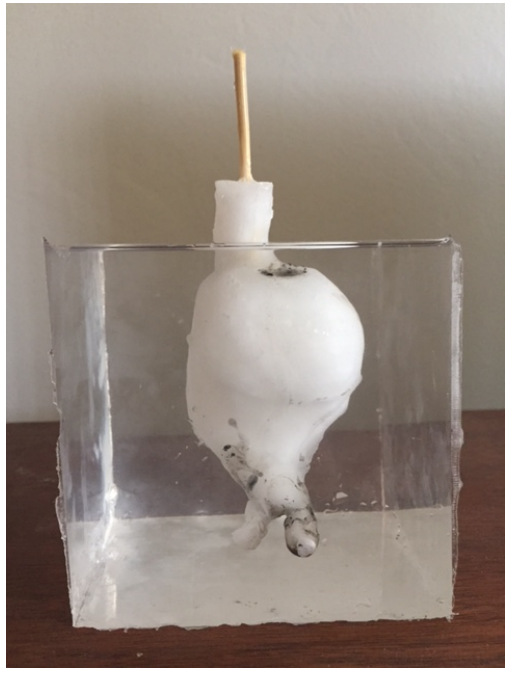

Figure 21. Front view of the wax inside the PDMS mold

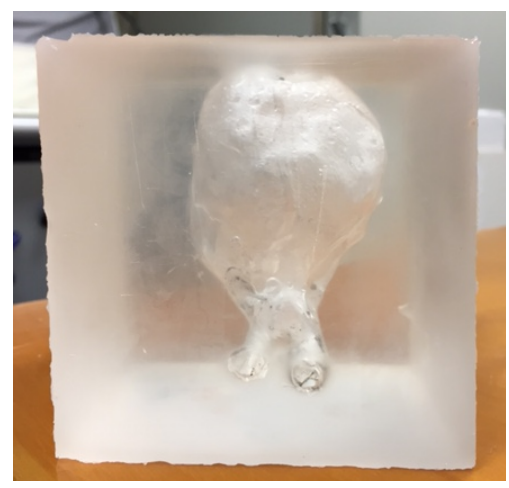

Figure 23. Front view of the hollow PDMS mold

The PDMS mold and wax model were placed in boiling water for 30 minutes to remove the wax from the mold and leave a hollow cavity. The wax was melted from the inside of the mold, but some adhered to the surface, resulting in a translucent appearance instead of 
a clear appearance. A razor blade was used to scrape of the wax from the surface of the mold. Gasoline was also used to dissolve the paraffin wax, and allow for easier removal. The paraffin wax is a non-polar substance due to its chain of hydrocarbons. Gasoline is a non-polar solvent, and for this reason was used to remove the paraffin wax from the PDMS mold. The final aneurysm mold used in the flow visualization trials can be seen in Figures 22 and 23.

\subsection{Flow Visualization}

\subsubsection{Flow Visualization Set Up}

In this study, the steady state flow of water was used to simulate the pumping of blood from the heart. Pulsatile flow was outside the scope of this study. Cardiac output, seen in Equation 8, was used to estimate the correct pump for flow visualization. In the equation, $\mathrm{CO}$ is the cardiac output in Liters per minute, $\mathrm{SV}$ is the stroke volume in Liters per beat, and HR is the heart rate in beats per minute (bpm)

Equation 8: $\quad C O=S V H R$

According to a study from Spodick et al, the average resting heart rate of a human is between 50 and $90 \mathrm{bpm}$ [32]. The normal stroke volume in the human heart is 70 $\mathrm{mL} /$ beat [18]. Cardiac output is given in $\mathrm{mL} / \mathrm{min}$, which can be converted to US Gallons/minute to find a suitable pump. 
Minimum:

$\mathrm{CO}=(50$ beats $/ \mathrm{min})(70 \mathrm{ml} / \mathrm{beat})=3500 \mathrm{ml} / \mathrm{min}$

$3.5 \mathrm{~L} / \mathrm{min}=55.47 \mathrm{gal} / \mathrm{hour}$

Maximum:

$\mathrm{CO}=(90$ beats $/ \mathrm{min})(70 \mathrm{ml} / \mathrm{beat})=6300 \mathrm{ml} / \mathrm{min}$

$6.3 \mathrm{~L} / \mathrm{min}=99.86 \mathrm{gal} / \mathrm{hour}$

From these calculations, the suitable rate for the pump must be between 55 and 100 Gallons/hour. To find the flow rate of the pump, tubing was connected, and the time required to fill $1 \mathrm{~L}$ was measured for ten trials. The average time was found to be 15.094 \pm 0.5568 seconds. Equations 9 and 10 were used to find the flow rate of the aneurysm construct.

Equation 9:

$\frac{1 L}{15.094 s}=\frac{0.264172 \text { gallons }}{15.094 s}$

Equation 10:

$$
\frac{0.01750178 \text { gallons }}{s}=\frac{63.0064 \text { gallons }}{1 \text { hour }}
$$

The flow rate for the pump was found to be 63.0064 gallons/hour \pm 2.33 gallons/hour, which is in between 55 and 100 gallons/hour. The pump was therefore suitable to use for the flow visualization trials. 
Tubing with an inner diameter of $5 / 8$ " and an outer diameter of $3 / 4$ " was press fit into the neck of the aneurysm model, and tubing with an inner diameter of 3/16" and an outer diameter of 5/16" was press fit into each of the legs of the aneurysm model. The tubing was sealed with clear silicone sealant to prevent leaking.

\subsection{Imaging Set Up}

The aneurysm model and tubing was placed on a bucket, and lighting was placed behind the PDMS to illuminate the model. An iPhone 6 was placed in front of the model to capture video of the flow visualization trials. The pump was placed in water, and the tubing attached to the legs of the aneurysm model was placed in an empty bucket to allow

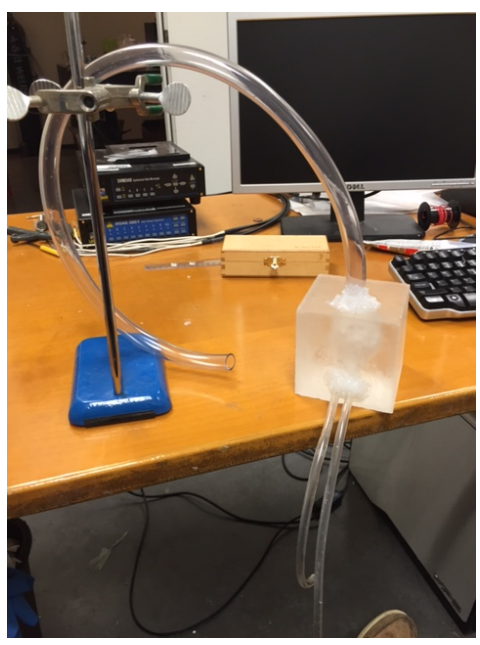

a.

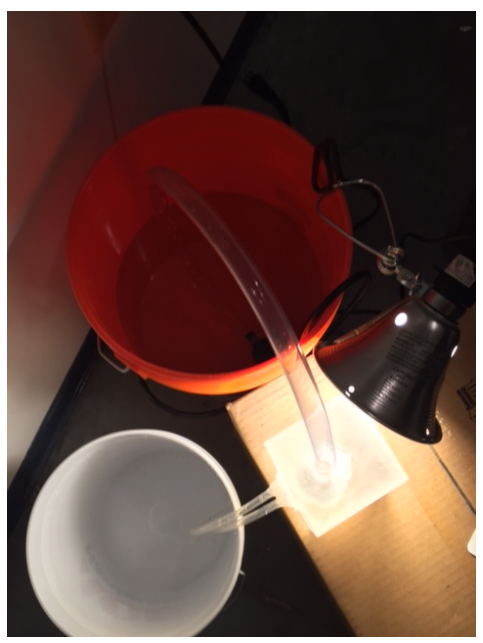

b.

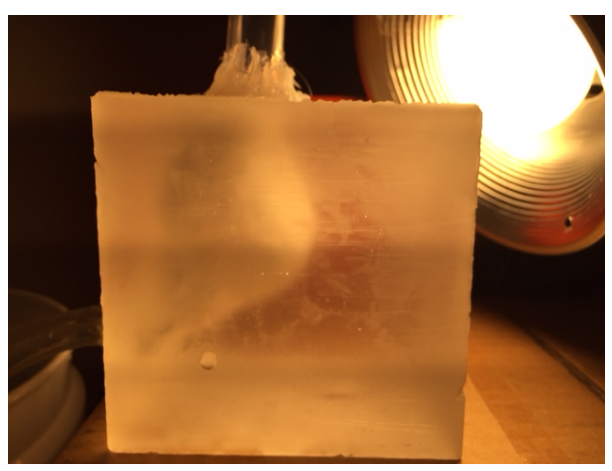

c.

Figure 24. (a) The tubing connected to the PDMS construct. (b) The set-up of the flow visualization. (c) The side of the PDMS construct where the videos were taken.

for drainage (Figure 24). The flow rate of the pump was measured at the same position where the construct would be for the flow visualization trials. It was assumed that the inlet of the vessel had the same flow rate as the outlet of the tubing. Flow was established 
in the aneurysm model, and the model was inverted to remove any bubbles. Slow motion video was taken as $1000 \mathrm{~mL}$ of red dye was injected into the pump.

Ten trials were administered to measure the time to fill one liter of water measured at the exit of the PDMS construct. The resulting times were averaged, and a value of 15.094 seconds per Liter was recorded. The flow rate of the pump was determined to be 6.62514 $\mathrm{x} 10^{-5} \mathrm{~m}^{3} / \mathrm{s}$ as seen in Appendix F. Flow rate is conserved throughout the model, so the flow rate at the exit can be used to find the velocity of the fluid at the entrance of the model. An average velocity of $26.035 \mathrm{~cm} / \mathrm{s}$ was determined, resulting in a maximum velocity of $52.07 \mathrm{~cm} / \mathrm{s}$, shown in Appendix F.

\subsection{Imaging Methods}

The still images collected from the videos of the flow visualization trials were used to determine the coordinates of the dye as it moved through the aneurysm model. The coordinates were determined using the following method, and this method was applied to every image in the trial.

1) Determine an origin (common feature) in COMSOL Multiphysics ${ }^{\circledR}$ and ImageJ

2) Convert pixels to centimeters

3) Find the absolute distance from the origin in $\mathrm{x}$ and $\mathrm{y}$ coordinates

4) Calculate the velocity between image frames

5) Measure the angle of rotation

6) Apply the coordinate transformation equations to find equivalent coordinates in COMSOL Multiphysics® 
This method was adapted from a procedure developed by Greinke [16], Willis [17], and Knauer [18]. The velocity and position of the dye was determined by comparing the furthest downstream pixel value in one frame to the furthest downstream pixel on the preceding frame.

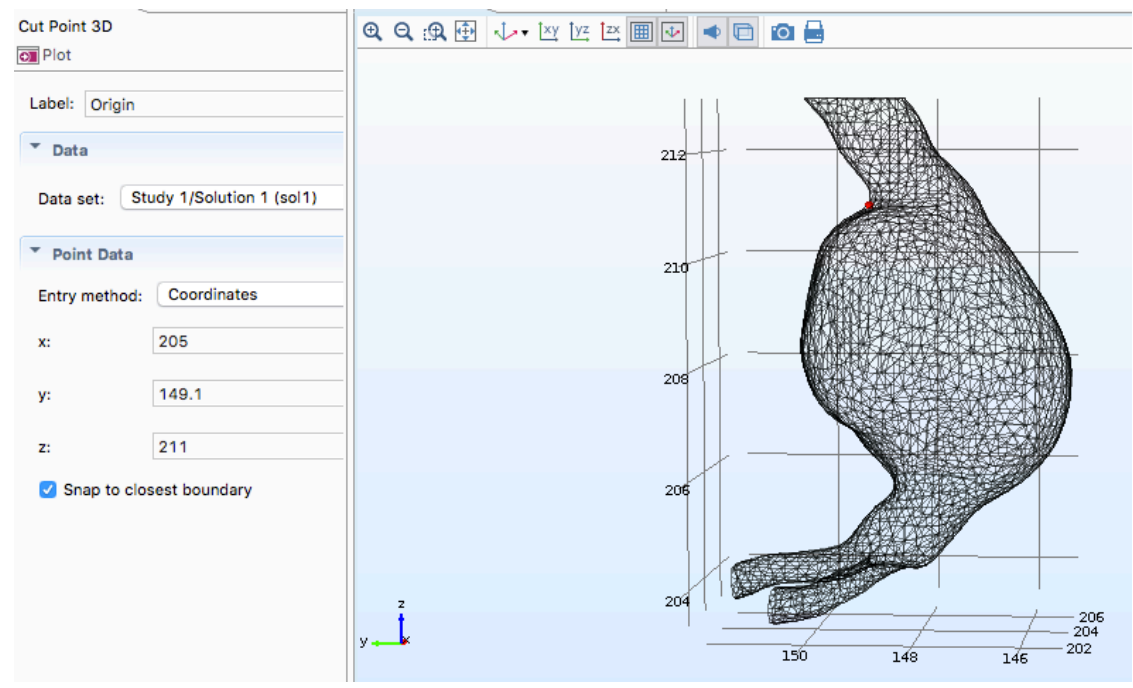

Figure 25. Origin of the COMSOL Multiphysics ${ }^{\circledR}$ model (red dot). The coordinate system of the COMSOL Multiphysics ${ }^{\circledR}$ model is located in the lower left corner of the graphics window.

The origin was selected where the neck of the aneurysm connects with the sac of the aneurysm, as this could easily be identified in both the computer model and the physical model, seen in Figure 25 and 26.

The coordinates were collected in COMSOL Multiphysics ${ }^{\circledR}$ as x, y, and z coordinates. The origin point was used to align the coordinate systems, and be a reference point for the physical model. 
Videos of the flow visualization trials were captured. The videos were then converted to image sequences. The number of frames in the videos was determined, and the number of frames was divided by the lengths of the videos to find the number of frames per second. Screenshots of the videos were taken every ten frames, giving a known time difference

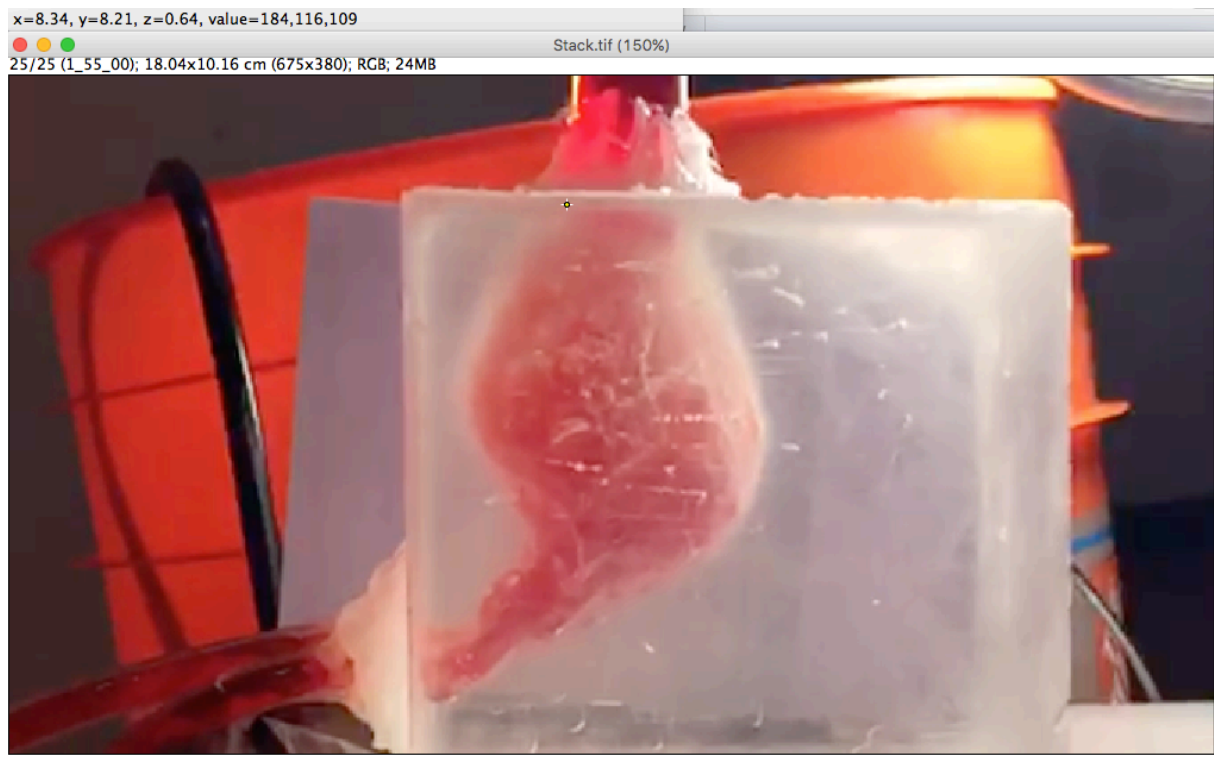

Figure 26. The origin of the PDMS construct. The coordinate system is set up with $x=0, y=0$ in the lower left corner of the image.

between frames. The images that contained the dye were collected and analyzed using Image J software. A detailed description can be found in Appendix H. The collection of images was imported into ImageJ, and was merged into a stack. A scale was set using the length of the PDMS mold $(10 \mathrm{~cm})$. This allowed the coordinates in Image $\mathrm{J}$ to represent centimeters and not pixels. The point of interest in each image was the location of the red dye. In ImageJ, a threshold was chosen so that the red dye was more easily visible. The stack was then converted back into individual images. The location of the furthest dye point was collected using $\mathrm{x}$ and $\mathrm{y}$ coordinates. The initial point where the dye entered neck of the aneurysm was denoted point "a". In the following frame, the furthest dye point was denoted point "b". This was replicated for each frame and subsequent dye point 
until the dye had exited the legs of the aneurysm, culminating at point " $h$ ". The different trails were denoted by numbers, so point "a" on trial one was denoted "T1a".

The absolute coordinates were determined by taking the image coordinates and then subtracting the $\mathrm{x}$ and $\mathrm{y}$ coordinates from the coordinates of the model origin. The absolute coordinates represented the distance between the pixel at the front of the dye and the origin. The absolute coordinates were used to determine the change of position of the dye front. The absolute coordinates of the preceding image were subtracted from the absolute coordinates of the image of interest. This change of position was then used to calculate the velocity of the dye between the two images. The time between the images was found by taking the total length of the video divided by the time of the video. The slow motion videos taken on the iPhone 6 were shot at 240 frames per second. The region of interest where the dye initially entered the aneurysm construct until it left the construct was 3 seconds long. The video therefore had 720 frames. The time per frame was 3 seconds divided by 720 frames, or 0.00416667 seconds/frame. There were ten frames between each captured image. The velocity was calculated by dividing the change in position by the time between each image, $(0.00416667 * 10)$. The outcome is values of $x$ and $y$ for the experimental model in terms of absolute coordinates and velocity.

The angle of rotation was determined in order to properly transform the coordinate system from the ImageJ coordinates into the COMSOL Multiphysics ${ }^{\circledR}$ coordinates. A detailed description can be found in Appendix I. Screenshots of the COMSOL Multiphysics ${ }^{\circledR}$ geometry were imported into ImageJ. The angle of the neck of the 
aneurysm and the angle of the legs of the aneurysm were determined using the angle tool. The image of the PDMS mold was imported into ImageJ. The angle tool was used to determine the angle of the neck of the aneurysm and the legs of the aneurysm. The angle of the neck of the PDMS model was subtracted from the angle of the neck of the COMSOL Multiphysics ${ }^{\circledR}$ model. The result was the difference in angle between the COMSOL Multiphysics ${ }^{\circledR}$ model and PDMS model. The angle of the legs of the PDMS model was subtracted from the angle of the legs of the PDMS model. The result was the difference in angle between the COMSOL Multiphysics ${ }^{\circledR}$ model and the PDMS model. The difference in angle of the neck and the difference in angle of the legs were averaged, and the resulting angle was the angle of rotation used in the transformation equation.

To transform the PDMS coordinates to the COMSOL Multiphysics ${ }^{\circledR}$ coordinates, it is necessary to describe both of the coordinate systems. The coordinate system in ImageJ has its origin in the lower left corner of the image. The positive $\mathrm{x}$ - and positive $\mathrm{y}$-values are of a standard coordinate system. The y-coordinates in COMSOL Multiphysics ${ }^{\circledR}$ are the X-coordinates in the ImageJ model. The $\mathrm{z}$-coordinates in COMSOL Multiphysics ${ }^{\circledR}$ are the $y$-coordinates in the ImageJ model. The $\mathrm{x}$-coordinates in COMSOL Multiphysics ${ }^{\circledR}$ are the $\mathrm{z}$-coordinates in the ImageJ model. Whereas in the ImageJ model the $\mathrm{x}$-coordinates increase to the right, in the COMSOL Multiphysics® model, the ycoordinates increase to the left, as seen in Figures 25 and 26. 
The pixel coordinates were transformed to COMSOL Multiphysics® coordinates using the coordinate transformation equations adapted from studies by Grienke [16], Willis [17], and Knauer [18]. The equations can be seen in Equation 11 and 12:

Equation 11: $x_{r o t}=\left[\left(\left(x-C_{x}\right) \cos \theta\right)-\left(\left(y-C_{y}\right) \sin \theta\right)\right]+C_{x}$

Equation 12: $y_{\text {rot }}=\left[\left(\left(x-C_{x}\right) \sin \theta\right)+\left(\left(y-C_{y}\right) \cos \theta\right)\right]+C_{y}$

Where $\mathrm{x}_{\text {rot }}$ and $\mathrm{y}_{\text {rot }}$ represent coordinates in the COMSOL Multiphysics ${ }^{\circledR}$ model, $x$ and $y$ represent the point of interest in the image, $C_{x}$ and $C_{y}$ represent the COMSOL Multiphysics ${ }^{\circledR}$ origin coordinates, and $\theta$ represents the angle of rotation measured from the experimental model.

The result of the transformation equation were $\mathrm{x}$ and $\mathrm{y}$ coordinates (COMSOL Multiphysics ${ }^{\circledR}$ y and $z$ coordinates) that described the farthest moving pixel within the model. Because the $\mathrm{x}$ coordinates in Image $\mathrm{J}$ increased in value to the right, and the corresponding COMSOL Multiphysics ${ }^{\circledR}$ y coordinates increased to the left, it was necessary to invert the ImageJ x coordinates (COMSOL y coordinates). The COMSOL Multiphysics ${ }^{\circledR}$ y-coordinates were mirrored around the y value at the COMSOL Multiphysics ${ }^{\circledR}$ origin. 
The outputs of this sequence were $\mathrm{x}$ and y coordinates within COMSOL Multiphysics ${ }^{\circledR}$ that represented the farthest moving pixel within the model, as well as the associated velocities. These coordinates were then input into the COMSOL Multiphysics ${ }^{\circledR}$ model to compare velocity measurements

\subsection{Velocity Values}

The coordinates were input into COMSOL Multiphysics ${ }^{\circledR}$ following the directions seen in Appendix I. A 3D cut point was added to the COMSOL Multiphysics ${ }^{\circledR}$ model. The $\mathrm{x}$ and y coordinates transformed from the ImageJ coordinate system to the COMSOL Multiphysics ${ }^{\circledR}$ coordinate system were input into COMSOL Multiphysics ${ }^{\circledR}$ as y- and zcoordinates, respectively. The $\mathrm{x}$-value used as the origin was input as the $\mathrm{x}$-value for each $3 \mathrm{D}$ cut point. The $3 \mathrm{D}$ cut point was plotted. This process was repeated for all coordinates. To obtain the velocity at the 3D cut point, a point evaluation was used for each 3D cut point. The velocity from this approach was the maximum velocity at that point.

\subsubsection{Mesh Confidence Study}

To find a COMSOL Multiphysics ${ }^{\circledR}$ mesh that provided accurate velocity values, a mesh confidence study was performed. The COMSOL Multiphysics® model was amended so that instead of a turbulent model, a creep flow model was used to model the fluid flow. This was done to allow for quicker COMSOL Multiphysics ${ }^{\circledR}$ computations. A point within the COMSOL Multiphysics® model was chosen to evaluate the velocity at 
different mesh accuracy levels. The point T3b, the second velocity point on third fluid visualization trial, was selected. The maximum velocity was determined for three mesh accuracy levels as seen in Table 1 .

Table 1: COMSOL Multiphysics Mesh levels and corresponding number of elements

\begin{tabular}{|l|l|}
\hline COMSOL Multiphysics Level & Number of elements \\
\hline Normal & 62,280 \\
\hline Fine & 162,745 \\
\hline Finer & 279,287 \\
\hline
\end{tabular}

Table 2. Velocity Values used in the Mesh Confidence Study

\begin{tabular}{|c|c|}
\hline & Point \\
\hline Predefined Mesh & Maximum Velocity $(\mathrm{cm} / \mathrm{s})$ \\
\hline Normal & 53.159 \\
\hline Fine & 58.572 \\
\hline Finer & 60.063 \\
\hline Extra Fine & N/A \\
\hline Extremely Fine & N/A \\
\hline
\end{tabular}

The COMSOL Multiphysics ${ }^{\circledR}$ model did not converge for Extra Fine and Finest, so they were not evaluated. The velocity at two levels were compared, and the process was completed when there was a difference of less than $5 \%$ between two mesh levels as seen in Tables 1 and 2. The process is detailed in Appendix J. The mesh was used for the COMSOL Multiphysics $®$ evaluation. 


\subsubsection{COMSOL Multiphysics ${ }^{\circledR}$ model}

The COMSOL Multiphysics ${ }^{\circledR}$ model was created following the directions in Appendix K. A 3D component was created. Parameters for the model were input. The density and viscosity of water were used to replicate the PDMS model. Density $($ rho $)=1000 \mathrm{~kg} / \mathrm{m}^{3}$ and viscosity (vis) $=0.8 \mathrm{cP}$. Gage pressure was used in the model, so the output pressure $\left(\mathrm{P} \_\right.$out $)=0 \mathrm{~Pa}$. The flow rate of the pump was used to determine the mass flow rate; rho*flow rate $\left[6.62514 \times 10^{-5} \mathrm{~m}^{3} / \mathrm{s} * 1000 \mathrm{~kg} / \mathrm{m}^{3}\right] .6 .6251 \times 10^{-2} \mathrm{~kg} / \mathrm{s}$ was input as the parameter for the inlet. The IGES file of the aneurysm model was imported into COMSOL Multiphysics ${ }^{\circledR}$. Laminar flow physics were added to the model. Density and viscosity were selected as the fluid properties. Under the properties of laminar flow, a laminar model was selected. The Reynolds number was found using Equation 7.

In this equation, $\rho=1000 \mathrm{~kg} / \mathrm{m}^{3}, v=52.07 \mathrm{~cm} / \mathrm{s}, \mathrm{D}=1.89 \mathrm{~cm}$, and $\mu=0.89 \mathrm{cP}$. Therefore the

Table 3. Percent differences of the velocity values used in the Mesh Confidence Study

\begin{tabular}{|l|r|}
\hline Percent Difference & \\
\hline Predefined Mesh & \\
\hline Normal to Fine & 10.18265957 \\
\hline Fine to Finer & 2.545584921 \\
\hline Finer to Extra Fine & \\
\hline
\end{tabular}

Reynolds number is 11,060 . From this number, the flow should be represented as turbulent flow. However, when creating the COMSOL Multiphysics ${ }^{\circledR}$ model, the aneurysm model was unable to converge using turbulent flow. Laminar flow was chosen instead of turbulent flow. 
The top of the neck of the aneurysm was chosen as the inlet, and the ends of the legs of the aneurysm were chosen as the outlets. In the inlet, the boundary condition selected was velocity. Mass flow rate was input as input parameter. The boundary condition for the outlets was set to pressure, and $\mathrm{P} \_$out was input as the pressure condition. As seen in Tables 2 and 3, a Fine mesh was chosen for the COMSOL Multiphysics ${ }^{\circledR}$ model. A time dependent study was added to the model, and the study was computed.

\subsubsection{COMSOL Multiphysics ${ }^{\circledR}$ Validation using normal blood vessel}

A simplified blood vessel model was created to validate the COMSOL Multiphysics ${ }^{\circledR}$ results. Fluid velocities and wall shear stresses for a 3D steady state model of an artery were estimated through COMSOL Multiphysics ${ }^{\circledR}$, and the results were compared to theoretical values. The given information for this model can be seen in Table 4.

Table 4. Given information for COMSOL Multiphysics® validation study

\begin{tabular}{|c|c|c|c|c|}
\hline Vessel & $\begin{array}{c}\text { Diameter } \\
(\mathrm{mm})\end{array}$ & $\begin{array}{c}\text { Length } \\
(\mathrm{cm})\end{array}$ & $\begin{array}{c}\text { Wall } \\
\text { thickness } \\
(\mathrm{mm})\end{array}$ & $\begin{array}{c}\text { Average } \\
\text { Velocity }(\mathrm{cm} / \mathrm{s})\end{array}$ \\
\hline Artery & 5 & 10 & 0.8 & 30 \\
\hline
\end{tabular}

The Hagen-Poiseuille equation was used to determine the theoretical pressure drop between the inlet and outlet, seen in Equation 13. It was assumed that there was no slip on the walls of the vessel and outet pressure was zero. Here the flow rate $\mathrm{Q}=$ (velocity)(Area), radius $R=2.5 \mathrm{~mm}$, velocity $\mathrm{v}=30 \mathrm{~cm} / \mathrm{s}$, outlet pressure $\mathrm{P}_{2}=\mathrm{OPa}$, viscosity $\mu=3 \mathrm{cP}$, and length $\mathrm{L}=10 \mathrm{~cm}$. The resulting pressure for the inlet pressure, $\mathrm{P}_{1}$, 
was found to be 115.2 $\mathrm{Pa}$. The theoretical wall shear stress was calculated using Equation 14. Here inlet pressure $P_{1}=115.2 \mathrm{~Pa}$, outlet pressure $P_{2}=0 \mathrm{~Pa}$, radius $R=2.5 \mathrm{~mm}$, and length $\mathrm{L}=10 \mathrm{~cm}$. The resulting average shear stress was found to be $1.44 \mathrm{~Pa}$.

Equation 13: $\quad Q=\frac{\pi R^{4}\left(P_{1}-P_{2}\right)}{8 \mu L}$

Equation 14: $\quad \tau_{w}=\frac{\left(P_{1}-P_{2}\right) R}{2 L}$

Table 5. Parameters used in COMSOL Multiphysics ${ }^{\circledR}$ validation

Parameters
\begin{tabular}{|l|l|l|l|}
\hline Name & Expression & Value & Description \\
\hline D & $5[\mathrm{~mm}]$ & $0.005 \mathrm{~m}$ & Diameter \\
L & $10[\mathrm{~cm}]$ & $0.1 \mathrm{~m}$ & Length \\
wt & $0.8[\mathrm{~mm}]$ & $8 \mathrm{E}-4 \mathrm{~m}$ & Wall thickness \\
v & $30[\mathrm{~cm} / \mathrm{s}]$ & $0.3 \mathrm{~m} / \mathrm{s}$ & Average Velocity \\
mu & $3[\mathrm{cP}]$ & $0.003 \mathrm{~Pa} \cdot \mathrm{s}$ & viscosity \\
rho & $1050\left[\mathrm{~kg} / \mathrm{m}^{\wedge} 3\right]$ & $1050 \mathrm{~kg} / \mathrm{m}^{3}$ & density \\
\hline P_in & $115.2[\mathrm{~Pa}]$ & $115.2 \mathrm{~Pa}$ & Inlet Pressure \\
\hline
\end{tabular}

A quarter cylinder was created in COMSOL Multiphysics ${ }^{\circledR}$ using the parameters in

Table 5. A symmetry boundary condition was used to simulate a cylindrical artery. The mesh used in the model was "Finer", and the mesh statistics can be seen in Appendix L. Creeping flow was used to model the fluid flow using steady state conditions. 
The resulting velocity slice plot can be seen in Figures 27 . To find the average velocity of the COMSOL Multiphysics® model, a line maximum was selected using the line

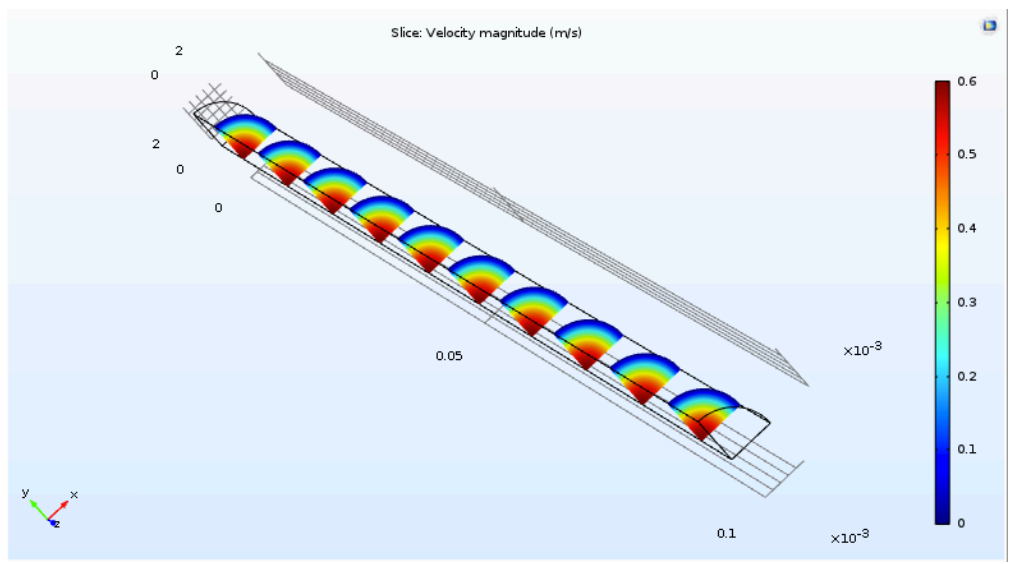

Figure 27. Velocity slice plot of artery for COMSOL Multiphysics ${ }^{\circledR}$ validation.

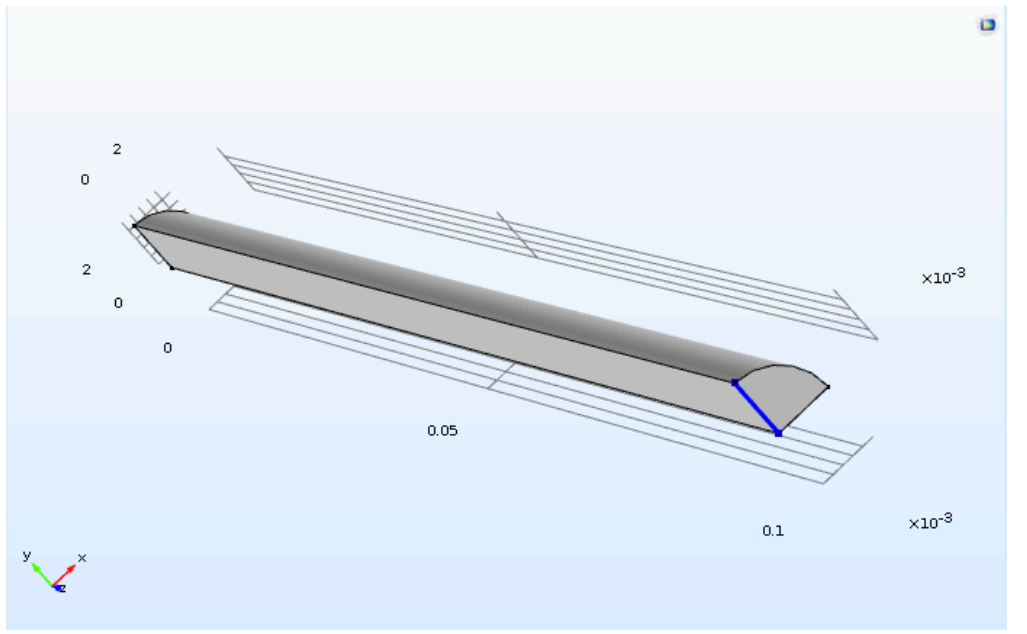

Figure 28. Line maximum used to find the maximum velocity of the artery fluid model

extending from the center of the vessel to the outer diameter, highlighted blue in Figure

28. The average velocity was found to be $30.059 \mathrm{~cm} / \mathrm{s}$, compared to the theoretical value of $30 \mathrm{~cm} / \mathrm{s}$. The percent error in this calculation was $0.1967 \%$. 
A slice plot of the shear stress is shown in Figure 29. To find the average wall shear stress of the COMSOL Multiphysics ${ }^{\circledR}$ model, a surface average was taken over the outer wall of the artery, as seen in Figure 30. The average wall shear stress was found to be $1.4402 \mathrm{~Pa}$, compared to the theoretical wall shear stress of $1.44 \mathrm{~Pa}$ found using

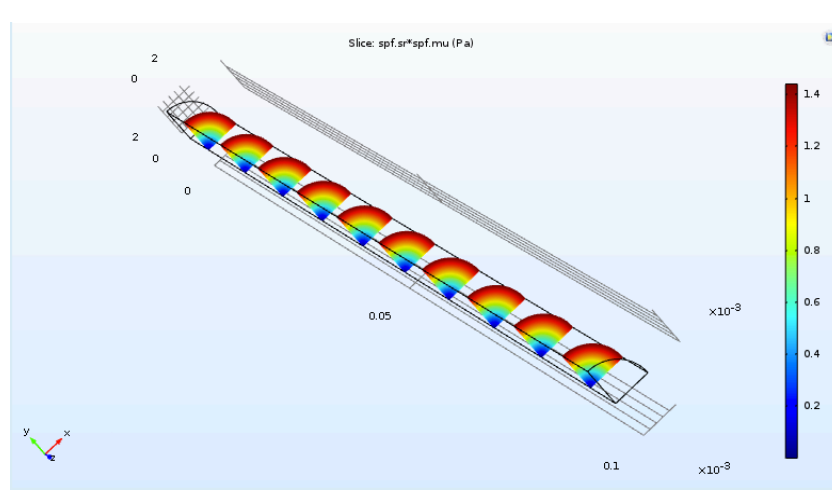

Figure 29. Shear stress slice plot of artery for COMSOL Multiphysics ${ }^{\circledR}$ validation

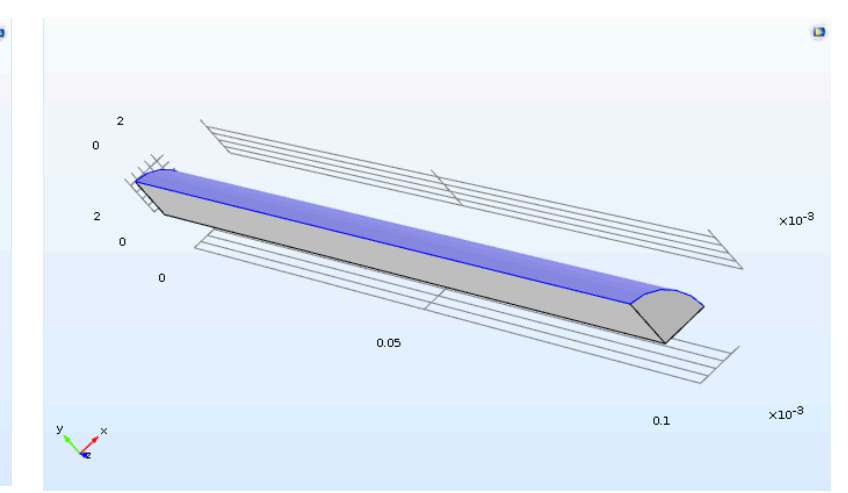

Figure 30. Surface selected to determine the average surface shear stress of the artery fluid model

Equation 15 . The percent error in this calculation was $0.0139 \%$ error. The goal of this COMSOL Multiphysics ${ }^{\circledR}$ validation study was to give show that the results given from the simple artery model are very accurate. This allows the values of the aneurysm model to be believed to be accurate as well. 


\section{CHAPTER 4: STATISTICAL ANALYSIS}

\subsection{Statistical Testing}

The statistical analysis comparing the experimental results and the COMSOL

Multiphysics ${ }^{\circledR}$ results was calculated using a Paired 2-Sample t-test using JMP software.

For the study, alpha was set to 0.05 , and the confidence interval was set to $95 \%$. The null hypothesis was that there was no difference between the two samples. The alternative hypothesis was that there was a significant difference between the samples. The output seen in Figure 31 shows a p-value of 0.0950 , which is greater than 0.05 . Since the p-value of 0.0950 is larger than 0.05 , we fail to reject the null hypothesis, as there is not significant statistical evidence to conclude a difference between the experimental velocity values and the COMSOL Multiphysics ${ }^{\circledR}$ velocity values.

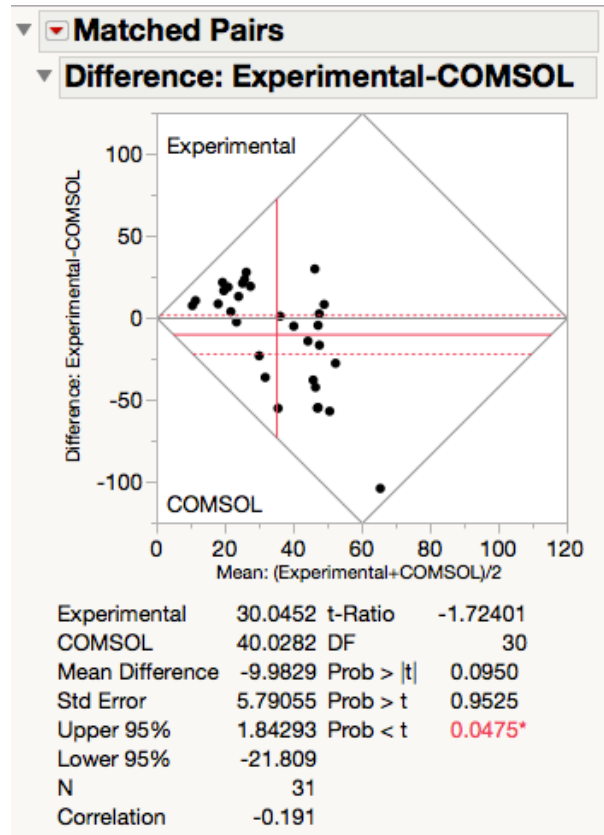

Figure 31. Paired 2-sample t-test results calculated using JMP software. 


\subsection{Comparative Results}

The following four graphs are comparisons between the experimental velocity values and the COMSOL Multiphysics ${ }^{\circledR}$ velocity values at equivalent coordinate points. Each graph is specific for the trial that was completed. Point "a" was located in the neck, and descending through the aneurysm sac to the leg of the aneurysm at point "h". As previously mentioned, the experimental velocity values were calculated by measuring the distance between the furthest downstream dye point in one frame and the furthest downstream dye point of the preceding frame. This distance was then divided by the time between the two frames. The resulting value is the velocity of the fluid at the designated coordinate point.

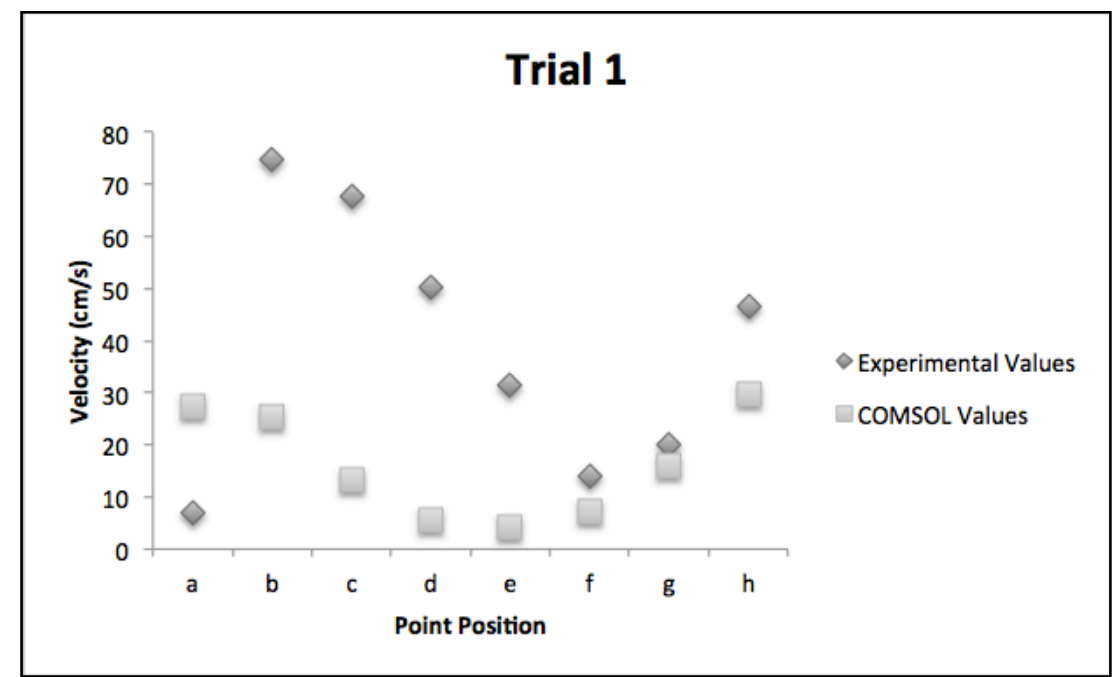

Figure 32. Scatter plot of Trial 1 comparing the COMSOL Multiphysics ${ }^{\circledR}$ and experimental velocity values at each coordinate point.

As seen in Figure 32, the velocity values of both the COMSOL Multiphysics ${ }^{\circledR}$ model and the experimental model follow a similar trend of higher velocities at the neck and the legs 
of the aneurysm, and reduced velocities in the aneurysm sac. The experimental velocity values also tend to be higher the COMSOL Multiphysics ${ }^{\circledR}$ velocity values.

As seen in Figure 33, Trial 2 follows a similar trend as Trial 1, in that the velocity values tend to be higher near the neck and the legs of the aneurysm for both the experimental

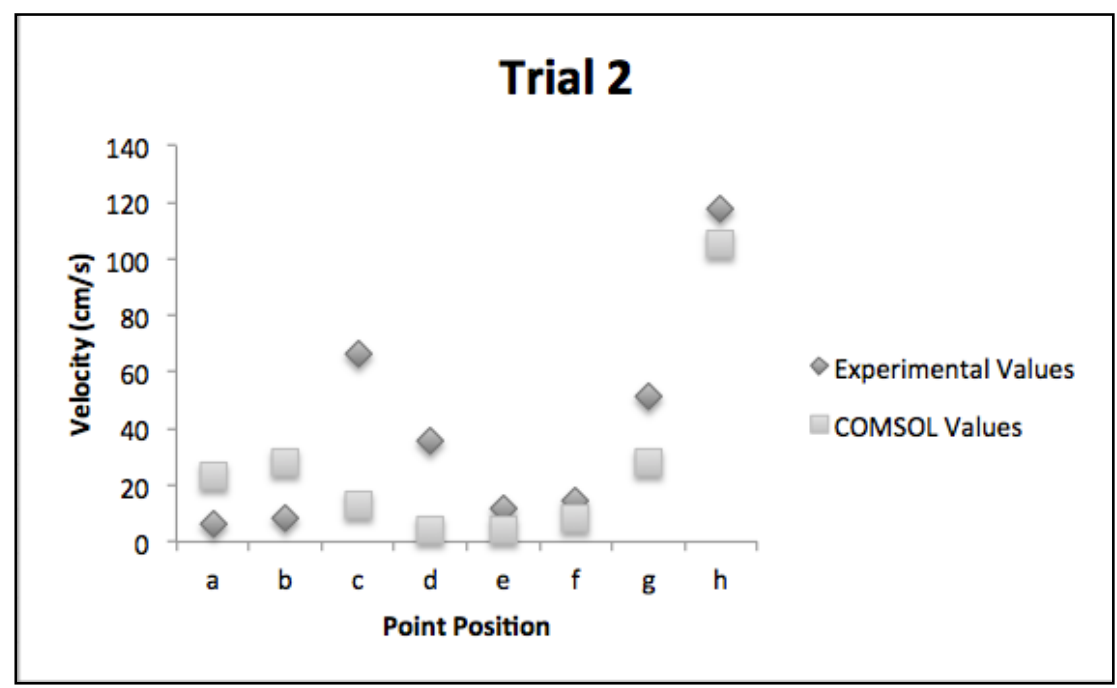

Figure 33. Scatter plot of Trial 2 comparing the COMSOL Multiphysics ${ }^{\circledR}$ and experimental velocity values at each coordinate point.

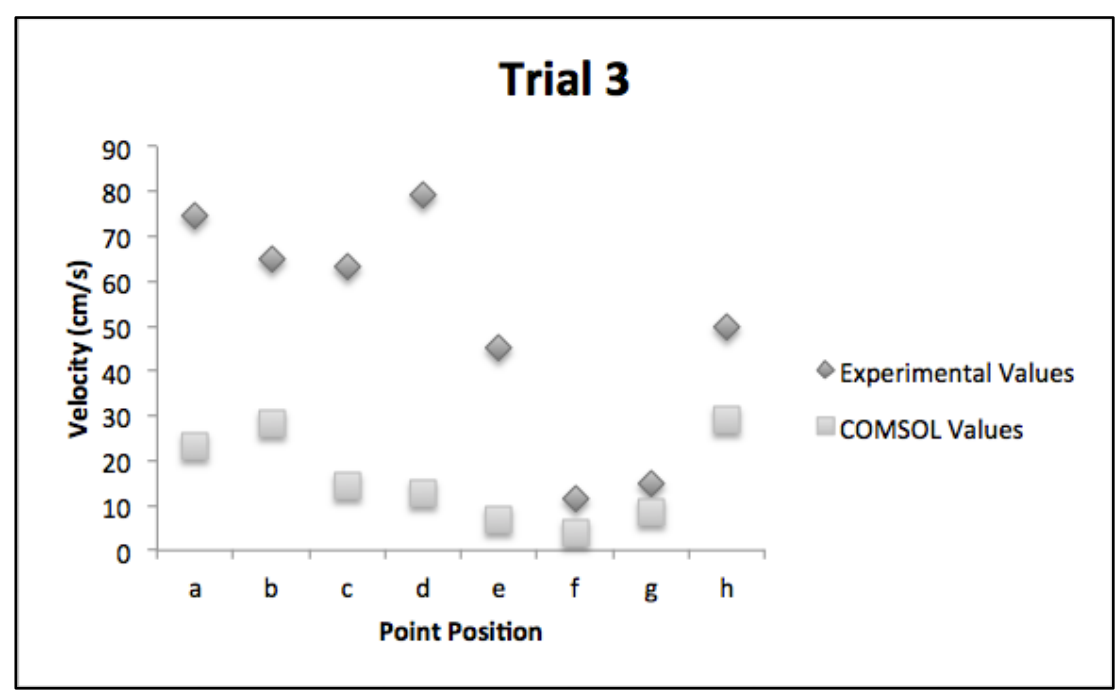

Figure 34. Scatter plot of Trial 3 comparing the COMSOL Multiphysics ${ }^{\circledR}$ and experimental velocity values at each coordinate point. 
and COMSOL Multiphysics ${ }^{\circledR}$ models. However, there are anomalies that may be due to inaccuracy in the model. The experimental values again tend to be higher than the COMSOL Multiphysics ${ }^{\circledR}$ model.

As seen in Figure 34, the difference between the experimental and COMSOL Multiphysics ${ }^{\circledR}$ velocity values is greater than Trial 1 and 2, however the same trend exists where the velocities are greater in the neck and legs of the aneurysm, and lower in the sac of the aneurysm. The experimental velocity values are all greater than the COMSOL Multiphysics ${ }^{\circledR}$ velocities.

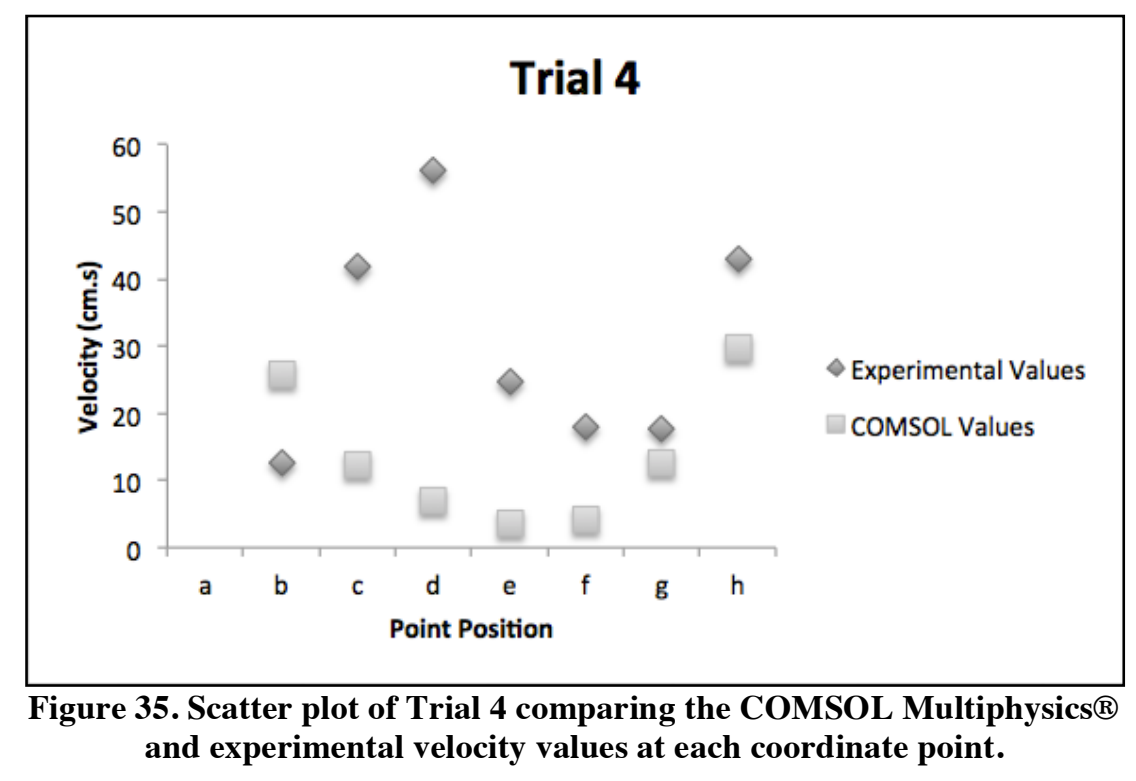

As seen in Figure 35, the results are not as distinct as Trials 1,2, and 3. The COMSOL Multiphysics ${ }^{\circledR}$ velocities follow the same trend as before with a greater velocity in the neck and legs of the aneurysm, and a reduced velocity in the sac of the aneurysm, however the experimental values appear more scattered. Again the experimental values tend to be greater than the COMSOL Multiphysics ${ }^{\circledR}$ values. 


\section{CHAPTER 5: DISCUSSION AND RESULTS}

\subsection{Interpretation of Experimental Model}

One aspect of this study was to create vasculature with an aneurysm in translucent polymer for flow visualization. The methods developed in this study used to create a physical model were effective and met the purpose of the study. It is possible and feasible to create a model of the abdominal aortic aneurysm from a CT scan. 3D printing was used to create a cheap but physiologically accurate model of the AAA. The PDMS construct created from the 3D printed aneurysm model was transparent enough to complete flow visualization studies, and was not damaged during experimentation. Another aspect of this study was to make the construct fully three dimensional with no seams. The aneurysm construct was manufactured using a single pour of PDMS. This method of manufacturing allowed for a hollow aneurysm chamber in a solid block of polymer, free from seams that could disrupt the flow of liquid during flow visualization testing.

This model could be used for the purposes of clinical testing, or for research and development. One application could be to create a physical model of a patients AAA. This model could then be used as a training tool for surgeons to practice the deployment of medical devices such as an endovascular stent graft, or it could be used as a reference during the surgery to give the surgeon a better idea of the complicated geometry of the aneurysm. This application would be for the purpose of reducing surgical error. A second 
application would be to use the aneurysm model to analyze a medical device during the testing and validation phase of development. The model would be able to give a qualitative analysis of the effect the medical device had on the blood flow within the aneurysm. A more quantitative analysis can also come from a computer model, and will be discussed in Section 5.2.

\subsection{Interpretation of COMSOL Multiphysics ${ }^{\circledR}$ Model and comparison study}

One of the goals of this study was to perform flow visualization and velocity characterization for comparison with finite element analysis. By using the velocities recorded from the PDMS construct, equivalent coordinate points were plotted in COMSOL Multiphysics ${ }^{\circledR}$, as previously shown, and these coordinate points were used to calculate the velocities at each point. Through a 2-Sample t-test, it was found that there was not evidence of a significant difference between the experimental model velocities and their equivalent COMSOL Multiphysics ${ }^{\circledR}$ velocities. The velocity results for each model appear to follow the correct trend of a larger velocity at the neck and the legs of the aneurysm, where the diameter of the vessel is smaller, and a decreased velocity in the sac of the aneurysm, where the diameter of the vessel increases. This is due to the equation of continuity [33]. 
There are a number of applications that a computational model can be used for. One application is to visualize the velocity profile that was seen in the experimental model,

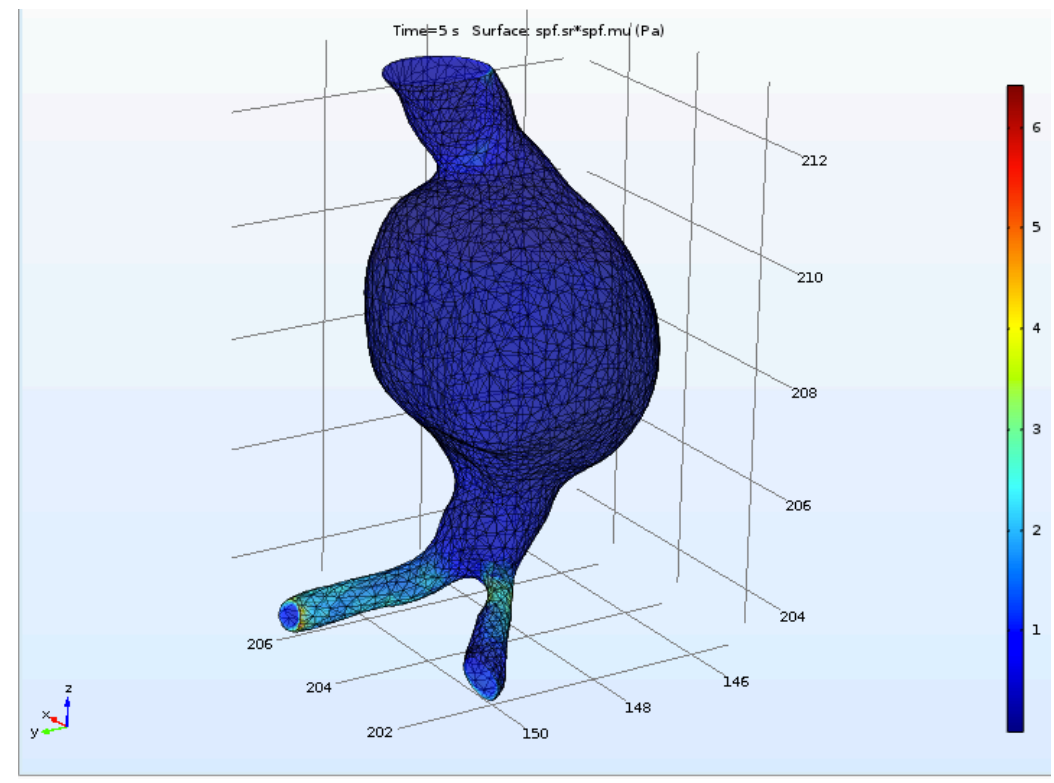

Figure 36. Wall shear stress in Pa for the aneurysm model plotted in COMSOL Multiphysics®

but with a more quantitative analysis. It is possible to see how the velocity is affected by the geometry of the aneurysm. In addition to analyzing characteristics that can be viewed in the experimental model, the computational model allows the user to analyze aspects that are not evident in the experimental model. Shear stress and pressure are both extremely important to understanding an aneurysm, and through the

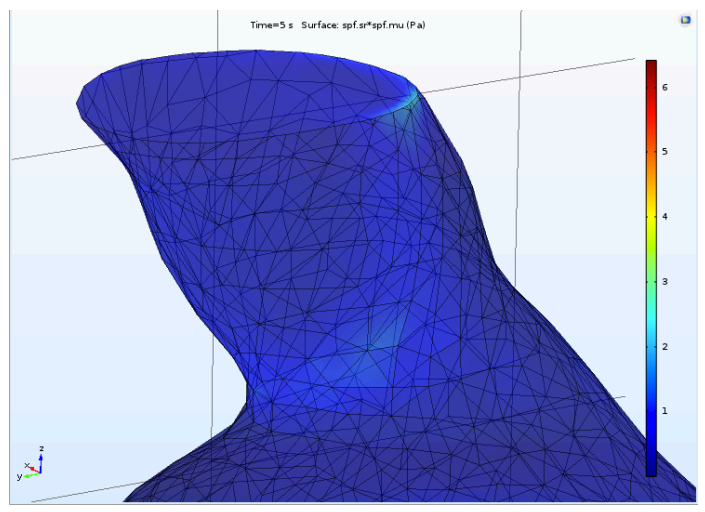

Figure 37. Zoomed in image of the wall shear stress in the neck of the COMSOL Multiphysics ${ }^{\circledR}$ aneurysm model 
computational model, it is possible to see how the fluid flow and the geometry of the aneurysm affect both the wall shear stress as well as the pressure on the wall of the vessel. In Figure 36, there are regions of high shear stress where the legs of the aneurysm branch from the sac of the aneurysm, as well as high stress

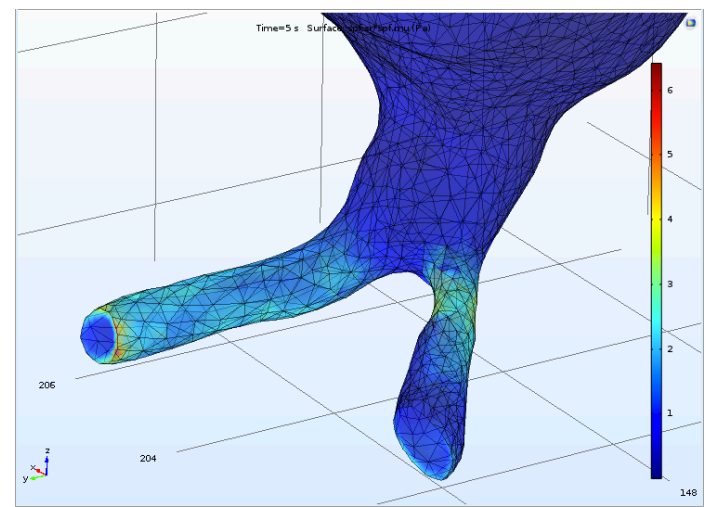

Figure 38. Zoomed in image of the wall shear stress in the legs of the COMSOL Multiphysics ${ }^{\circledR}$ aneurysm model

in where the neck of the aneurysm joins the sac of the aneurysm. There are also regions of low shear stress in the sac of the aneurysm. Both high and low shear stresses have an effect on the aneurysm. High shear stress can damage the endothelial cells, negatively impacting their ability to remodel [34]. Low shear stresses have been reported to be

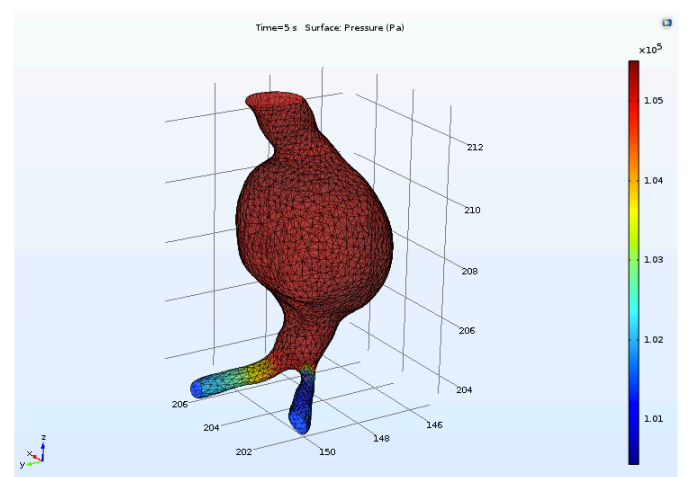

Figure 39. Plot of the wall pressure in Pa on the COMSOL Multiphysics $®$ aneurysm model.

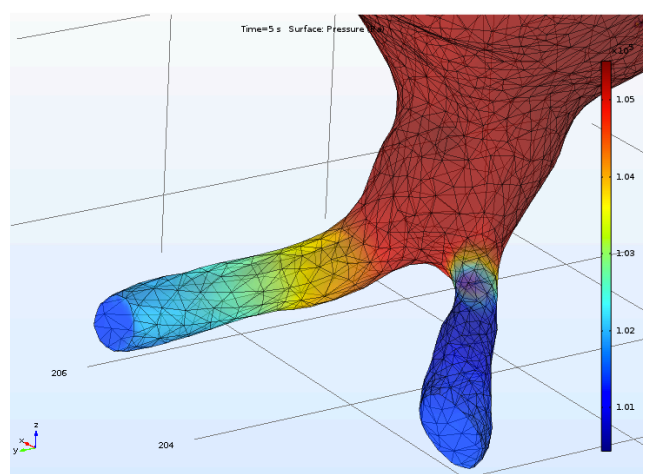

Figure 40. Zoomed in image of the wall pressure in the legs of the COMSOL Multiphysics ${ }^{\circledR}$ aneurysm model. 
related to aneurysm growth and rupture [34]. Figure 37 and 38 show a zoomed in image of both high shear stress regions of the aneurysm model. Figure 39 shows the pressure on the walls of the aneurysm, calculated using COMSOL Multphysics ${ }^{\circledR}$. It can be seen that in the neck and the sac of the aneurysm, the pressure is much higher than in the legs of the aneurysm, which has an impact on the aneurysm. Studies have shown that high pressure accelerates aneurysm expansion, and has been related to aneurysm rupture [35]. Figure 40 shows a zoomed in image of the pressure in the aneurysm model.

When analyzing the characteristics of the aneurysm model, it is important to be able to compare them to a healthy aorta. A model was created in COMSOL Multiphysics ${ }^{\circledR}$ to

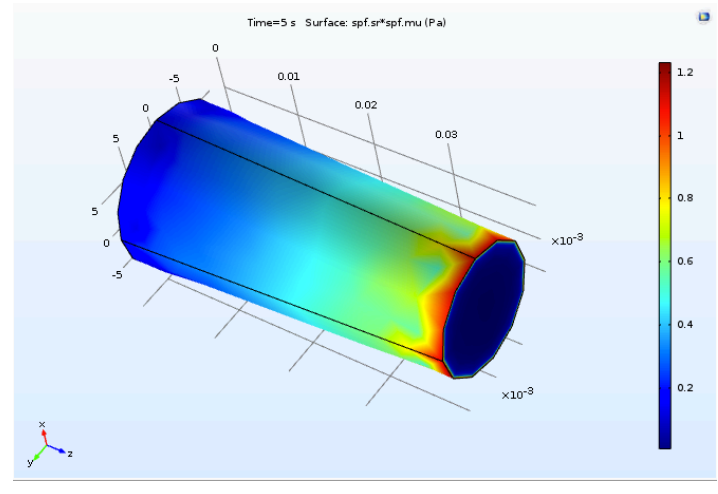

Figure 41. Wall shear stress values in $P a$ in a healthy aorta given the same inlet diameter, viscosity, density, and mass flow rate as the aneurysm model.

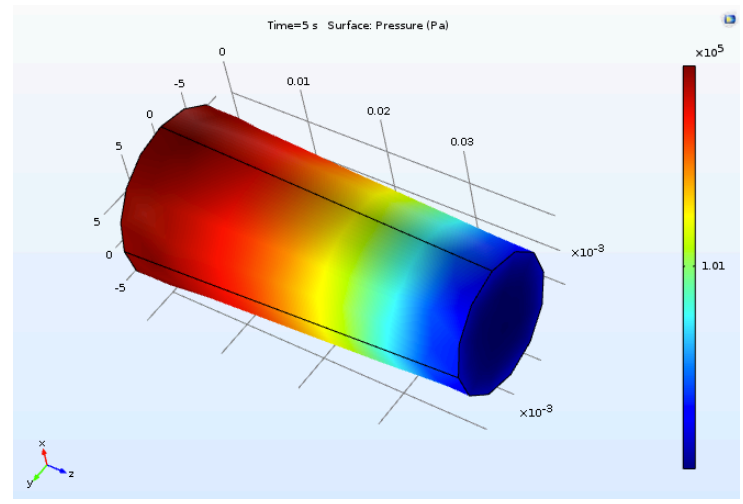

Figure 42. Pressure in Pa in a healthy aorta given the same inlet diameter, viscosity, density, and mass flow rate as the aneurysm model.

visualize the effect that the aneurysm had on the pressure and the wall shear stress. A vessel was created that had the same inlet diameter, viscosity, density, and mass flow rate as the aneurysm model. The outlet diameter was decreased to allow for a tapered vessel to replica the aorta as it descends through the abdomen before it splits to the iliac arteries. Figure 41 shows that the values of the wall shear stress are on the same magnitude as the aneurysm model, which gives confidence that the values calculated from the aneurysm 
model are correct. What is interesting though, for most of the healthy aorta, the wall shear stress values are less than $1 \mathrm{~Pa}$, where as in the aneurysm model, the wall shear stress values reach almost $6 \mathrm{~Pa}$ in some regions.

Figure 42 shows the values of the wall pressure in the healthy aorta. It can be seen that the pressure in the inlet matches the pressure in the aneurysm model. Also, the pressure change is much more gradual in the healthy aorta, as opposed to the aneurysm model which has high pressure for most of the aneurysm, and only drops in the legs.

The results of this study show that there is not a significant difference between the velocity values of the experimental model and the velocity values of the model created using COMSOL Multiphysics ${ }^{\circledR}$. While the results between the two models differed slightly, the in silico model validated the computational model. While a physical model is useful for various reasons previously explained, the computational model uses fewer resources to create and can output more results from a single study. A computational model can also be used when validating a medical device, for which physical testing would not be feasible.

\subsection{FDA use of computational models}

The validation of the aneurysm model using COMSOL Multiphysics® makes this protocol applicable to medical device testing. The Food and Drug Administration (FDA) is the regulatory body within the United States that is responsible for the approval and clearance to produce and market medical devices as procedures [36]. Testing is required 
to show the safety and efficacy of a device or procedure, however, the FDA recognizes computational models as a method of to evaluate this criteria. "Computational Fluid Dynamics and Mass Transport" is a publicly available document, which provides guidelines for testing and submission of a computational model [37]. This document includes information regarding the rationale for choosing the computational modeling approach as opposed to other approaches. The geometry, material properties, boundary/initial conditions, the simulation results, the quantities of interest, their implications for device safety and effectiveness are all included as reasons for using a computational model. This document does not provide requirements, it provides the suggestions that the FDA has for developing a thorough protocol for testing a medical device or procedure. The validation method used in this study was used to statistically validate a finite element analysis model and could hypothetically meet FDA requirements for submission. 


\section{CHAPTER 6: SUMMARY AND CONCLUSIONS}

\subsection{Contributions to Biomedical Engineering}

The results of this study contributed to the field of biomedical engineering by developing a method for creating a three-dimensional physical model of a CT scan, further establishing a novel technique for flow visualization, and successfully performing in silico validation. This model can be used both in a clinical and a research setting. The protocol described in this study could be applied to many different geometries to create complex physiological models, and since it was performed on a budget of less than $\$ 400$, it is a feasible form of validation testing. This study utilized a method of flow visualization developed in three previous studies. By further developing the method, three of the four studies have successfully used this method of flow to validate a computational model.

\subsection{Study limitations}

While the study was successful, there were a number of limitations associated with both the physical model and the computer model. In the physical model, a limitation was that there were ridges present in the $3 \mathrm{D}$ model due to the step sizes in the $3 \mathrm{D}$ printing. While these ridges were small, and may have had a small effect on the resulting flow during the flow visualization study, there were still present. Another limitation in the physical model was that the pressure was not controlled at the inlet and outlet. The outlet pressure in this 
study was atmospheric. Since the aneurysm construct was made of PDMS, the pressure inside the aneurysm increased as the fluid flowed through it, causing PDMS to distend, resulting in an increase in the flow rate. When measuring the time to fill $1 \mathrm{~L}$ using the tubing that connected to the inlet, the flow rate was found to be $7.7095 \times 10^{-5} \mathrm{~m}^{3} / \mathrm{s}$ however when measuring the time to fill $1 \mathrm{~L}$ at the outlet after the fluid flowed through the construct, the flow rate was found to be $6.62514 \times 10^{-5} \mathrm{~m}^{3} / \mathrm{s}$. The flow rate should be the same at the inlet and the outlet, so there is an issue with the model.

A limitation in the computer model was that the flow visualization study took a twodimension video and tried to apply the coordinates to a three-dimensional coordinate system. The z-coordinates in the COMSOL Multiphysics ${ }^{\circledR}$ model were estimated to be the center of the flow profile, as this would be where the highest velocity was. Another limitation of the computer model was that COMSOL Multiphysics ${ }^{\circledR}$ required a simplification of the original geometry to be able to process the file size. The mesh size used had the highest number of elements possible, as finer meshes caused the simulation to fail. The study was also run using laminar flow even though the characteristics called for turbulent flow. The simulation was unable to be completed using a turbulent flow, so laminar flow was chosen.

For the overall study, the limitations were associated with simulating in vivo conditions. Pulsatile flow would better mimic blood flow within the body, but was outside the scope of this study. Blood, or fluid with similar properties, would better emulate physiological condition for testing, but was also outside the scope of this project. The geometry used in 
this study was a simplified model of real patient anatomy. An assumption in this model was that the simplification of the model maintained the significant characteristics of the anatomical features.

There were a number of possible sources of error during this project. First, there was error when performing the flow rate calculations. An error of \pm 0.5568 seconds was calculated. While this was a small amount of error, it is possible that it compounded through the number of steps required to determine the COMSOL coordinates. Another source of error was when the threshold was applied to the image sequence in ImageJ. The threshold was applied manually, so there was human error involved in this process. Following the threshold step, the furthest dye point in each of the image sequences was located manually. This error could have been amplified at the entrance of the aneurysm. It is possible that the error at the inlet of the aneurysm causes a greater error for velocity calculations, as the points are closer together. By estimating the location of the furthest dye point, being off by a few pixels causes greater error than for images where the dye front is further apart, as is the case for the dye points in the sac and legs of the aneurysm. Another source of error was in the pump that was used. The flow rate was affected by the backflow of the water. This allowed for an inconsistent flow rate during the flow visualization trials. The last source of error was in the coordinate transformation. The coordinate transformation was accurate at most points, however, a number of coordinate points were not accurately transformed and ended up near the vessel wall, giving an artificially low velocity value 


\subsection{Future Works and Conclusions}

Minimally invasive surgeries are the most recent development to treat the complications of cardiovascular disease. Relevant applications include transcatheter aortic valve replacement (TAVR) and endovascular aneurysm repair (EVAR). As these procedures progress, it will become increasingly important to evaluate the medical devices, and visualize how they affect blood flow within the region of interest. This study provides the basis for developing a cheap and reusable, but physiologically accurate model to analyze blood flow.

In future studies, fluid with physiologically accurate properties should be incorporated. Water, which is a Newtonian fluid, was used in this study, however to properly model the in vivo characteristics, blood, which is a non-Newtonian fluid, should be used. This would be necessary when using this protocol for medical device testing. Within the physical model, the model could be extended to incorporate medical devices to visualize how the device affects the blood flow. This could be used both as practice for the surgeons implanting the medical devices in complicated geometries, as well as used for research and development purposes to verify that there are no adverse effects of the medical device.

In conclusion, this study used a CT scan to create an anatomically accurate physical model of an abdominal aortic aneurysm, as well as a computational model of the same aneurysm. The computational model was successfully validated using a novel method of 
flow visualization. The validation of the computational model is applicable to EVAR procedures, and this method could be applicable for different anatomies and other procedures within the aorta. 


\section{REFERENCES}

1 Ku, David N. "Blood Flow in Arteries." Annu. Rev. Fluid Mech 29 (1997): 399-434. Web.

2 Aggarwal, Sourabh et al. "Abdominal Aortic Aneurysm: A Comprehensive Review." Experimental \& Clinical Cardiology 16.1 (2011): 11-15. Print.

3 Finol, Ender A., and Cristina H. Amon. "Flow-induced Wall Shear Stress in Abdominal Aortic Aneurysms: Part I - Steady Flow Hemodynamics." Computer Methods in Biomechanics and Biomedical Engineering 5.4 (2002): 309-18. Web.

4 "Abdominal Aortic Aneurysms." Abdominal Aortic Aneurysm Diagnosis and Treatment Options. Society of Interventional Radiology, 2016. Web.

5 Schanzer, Andres, Dr. "Endovascular Repair of Abdominal Aortic Aneurysms." Endovascular Repair of Abdominal Aortic Aneurysms. Society for Vascular Surgery, n.d. Web.

6 Scherer, P. (1973) "Flow in Axisymmetrical Glass Model Aneurysms”, Journal of Biomechanics 6, 695-700. Web

7 Stehbens, W. (1974) "Flow Disturbances in Glass Models of Aneurysms at low Reynolds Numbers", Quarterly Journal of Experimental Physiology 59, 167-174. Web

8 Drexler, D. and Hoffman, A. (1985) "Steady Flow through several Aneurysm Models", In: Kuklinski, W. and Ohley, W., eds, Proceedings from the 11th Annual Northeast Bioengineering Conference, pp 147-150. Web

9 Budwig, R., Elger, D., Hooper, H. and Slippy, J. (1993) "Steady flow in Abdominal Aortic Aneurysm Models", ASME Journal of Biomechanical Engineering 115, 418423. Web

10 Taylor, T. and Yamaguchi, T. (1994) “Three-Dimensional Simulation of Blood Flow in an Abdominal Aortic Aneurysm - Steady and Unsteady Flow Cases", ASME Journal of Biomechanical Engineering 116, 89-97. Web

11 Asbury, C., Ruberti, J., Bluth, E. and Peattie, R. (1995) "Experimental Investigation of Steady Flow in Rigid Models of Abdominal Aortic Aneurysms", Annals of Biomedical Engineering 23, 29 - 39. Web

12 Peattie, R., Bluth, E., Ruberti, J. and Asbury, C. (1996) "Steady Flow in Models of Abdominal Aortic Aneurysms - Part I: Investigation of Velocity Patterns", Journal of Ultrasound in Medicine 15,679-688.

13 Peattie, R., Bluth, E., Ruberti, J. and Asbury, C. (1996) "SteadyFlow in Models of Abdominal Aortic Aneurysms - Part II: WallStresses and Their Implication for In Vivo Thrombosis and Rupture", Journal of Ultrasound in Medicine 15, 689 696. Web 
14 Schoephoerster, R., Oynes, F., Nunez, G., Kapadvanjwala, M. and Dewanjee, M. (1993) "Effects of Local Geometry and Fluid [18] Dynamics on Regional Platelet Deposition on Artificial Surfaces", Arteriosclerosis and Thrombosis 13, 1806 - 1813. Web

15 Bluestein, D., Niu, L., Schoephoerster, R. and Dewanjee, M. (1996) "Steady Flow in an Aneurysm Model: Correlation between Fluid Dynamics and Blood Platelet Deposition”, ASME Journal of Biomechanical Engineering 118, 280 - 286. Web

16 Greinke, Daniel C. "Fluid Flow Characterization in Rapid Prototyped Common Iliac Artery Aneurysm Molds." Thesis. California Polytechnic State University, San Luis Obispo, 2016. Print.

17 Willis, Rachel E. "Empirical Validation of an In Silico Model Predicting the Fluid Dynamics of an Iliac Artery Aneurysm." Thesis. California Polytechnic State University, San Luis Obispo, 2016. Print.

18 Knauer, Alexandra. "Fluid Flow Characterization And in Silico Validation in a Rapid Prototyped Aortic Arch Model." Thesis. California Polytechnic State University, San Luis Obispo, 2016. Print.

19 Lu, D., and G. S. Kassab. "Role of Shear Stress and Stretch in Vascular Mechanobiology." Journal of The Royal Society Interface 8.63 (2011): 1379-385. Web.

20 Rahimi, Saum A., MD, and Vincent Lopez Rowe, MD. "Abdominal Aortic Aneurysm." Abdominal Aortic Aneurysm: Practice Essentials, Background, Anatomy. WebMD, 28 Sept. 2015. Web.

21 Brady, A. R., F. G. R. Fowkes, S. G. Thompson, and J. T. Powell. "Aortic Aneurysm Diameter and Risk of Cardiovascular Mortality." Arteriosclerosis, Thrombosis, and Vascular Biology 21.7 (2001): 1203-207. Web

22 Hathcock, J. J. "Flow Effects on Coagulation and Thrombosis." Arteriosclerosis, Thrombosis, and Vascular Biology 26.8 (2006): 1729-737. Web.

23 Paszkowiak, J. J., and A. Dardik. "Arterial Wall Shear Stress: Observations from the Bench to the Bedside." Vascular and Endovascular Surgery 37.1 (2003): 47-57. Web.

24 Papaioannou, Theodoros G., and Christodoulos Stefanadis. "Vascular Wall Shear Stress: Basic Principles and Methods." Hellenic J Cardiol 46 (2005): 9-15. Web.

25 Raghavan, M.1., David A. Vorp, Michael P. Federle, Michel S. Makaroun, and Marshall W. Webster. "Wall Stress Distribution on Three-dimensionally Reconstructed Models of Human Abdominal Aortic Aneurysm." Journal of Vascular Surgery 31.4 (2000): 760-69. Web.

26 What Are the Signs and Symptoms of an Aneurysm?" National Institutes of Health. U.S. Department of Health and Human Services, 1 Apr. 2011. Web. 
27 Sudheendra, Deepak, MD, RPVI. "Abdominal Aortic Aneurysm." MedlinePlus Medical Encyclopedia. U.S. National Library of Medicine, 13 Aug. 2015. Web.

28 "How Is an Aneurysm Diagnosed?" National Institutes of Health. U.S. Department of Health and Human Services, 1 Apr. 2011. Web.

29 "Abdominal Aortic Aneurysms." Abdominal Aortic Aneurysm Diagnosis and Treatment Options. Society of Interventional Radiology, 2016. Web.

30 "DICOM Image Library." DICOM Image Library. Osirix, n.d. Web.

31 "Assembled Printrbot Simple." Printrbot. N.p., n.d. Web.

32 Spodick DH, Raju P, Bishop RL, Rifkin RD. Operational definition of normal sinus heart rate. Am J Cardiol. 1992;69:1245-6. Web

33 Srivastava, Anurag, Akshay Sood, S. Parijat Joy, and John Woodcock. "Principles of Physics in Surgery: The Laws of Flow Dynamics Physics for Surgeons - Part 1." Indian Journal of Surgery 71.4 (2009): 182-87. Web.

34 Boussel, L., V. Rayz, C. Mcculloch, A. Martin, G. Acevedo-Bolton, M. Lawton, R. Higashida, W. S. Smith, W. L. Young, and D. Saloner. "Aneurysm Growth Occurs at Region of Low Wall Shear Stress: Patient-Specific Correlation of Hemodynamics and Growth in a Longitudinal Study." Stroke 39.11 (2008): 2997-3002. Web.

35 Gadowski, Gregory R., David B. Pilcher, and Michael A. Ricci. "Abdominal Aortic Aneurysm Expansion Rate: Effect of Size and Beta-adrenergic Blockade." Journal of Vascular Surgery 19.4 (1994): 727-31. Web.

36 "Products and Medical Procedures." U.S. Food and Drug Administration. N.p., n.d. Web.

37 Reporting of Computational Modeling Studies in Medical Device Submissions." U.S. Food and Drug Administration. N.p., n.d. Web 


\section{APPENDICES}

\section{Appendix A: DICOM to STL}

Based off a tutorial found at https://vimeo.com/101017010

1. Import DICOM images into Osirix

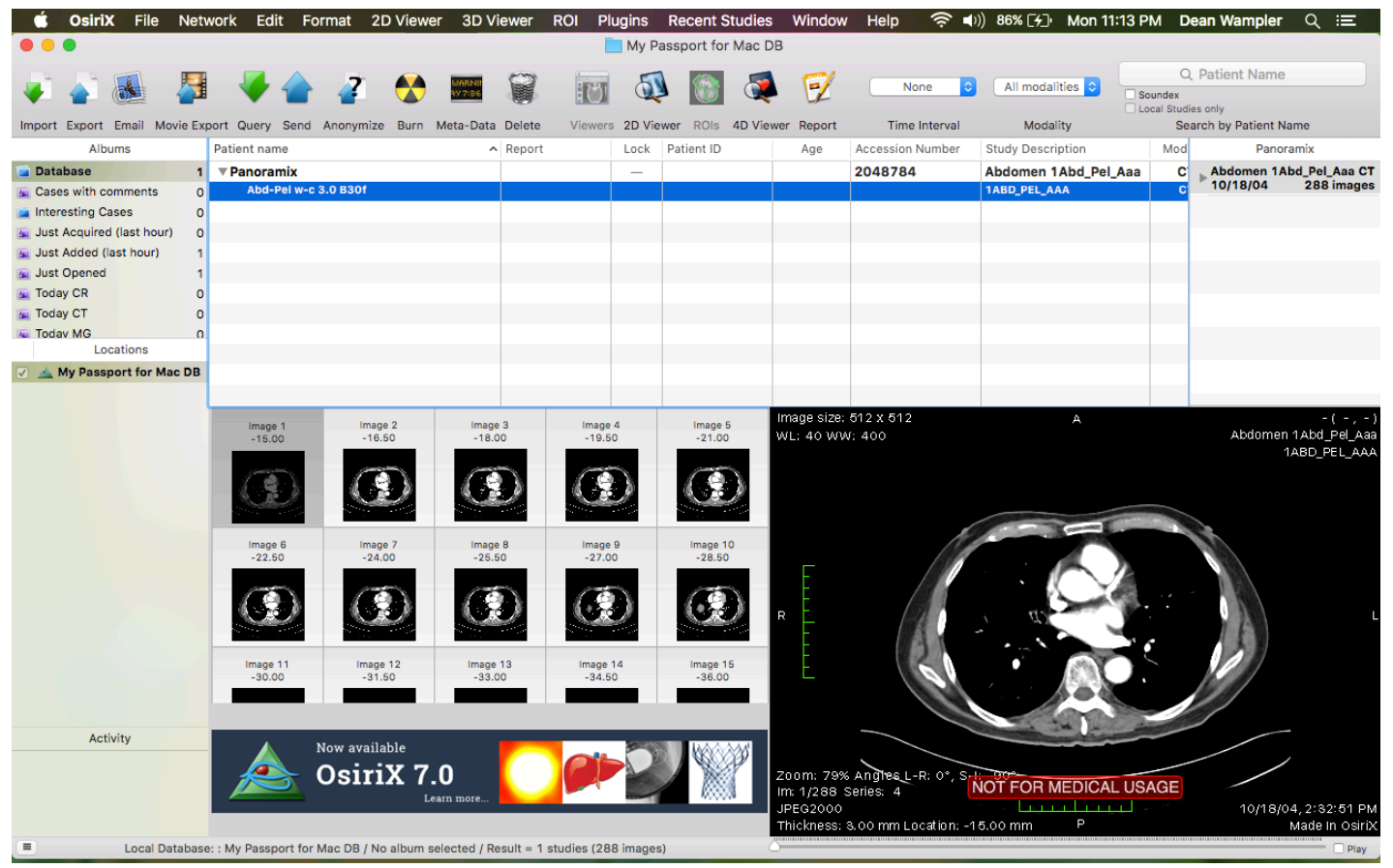


2. Adjust toolbar options to obtain proper tools are present

a. Format $>$ Customize Toolbars

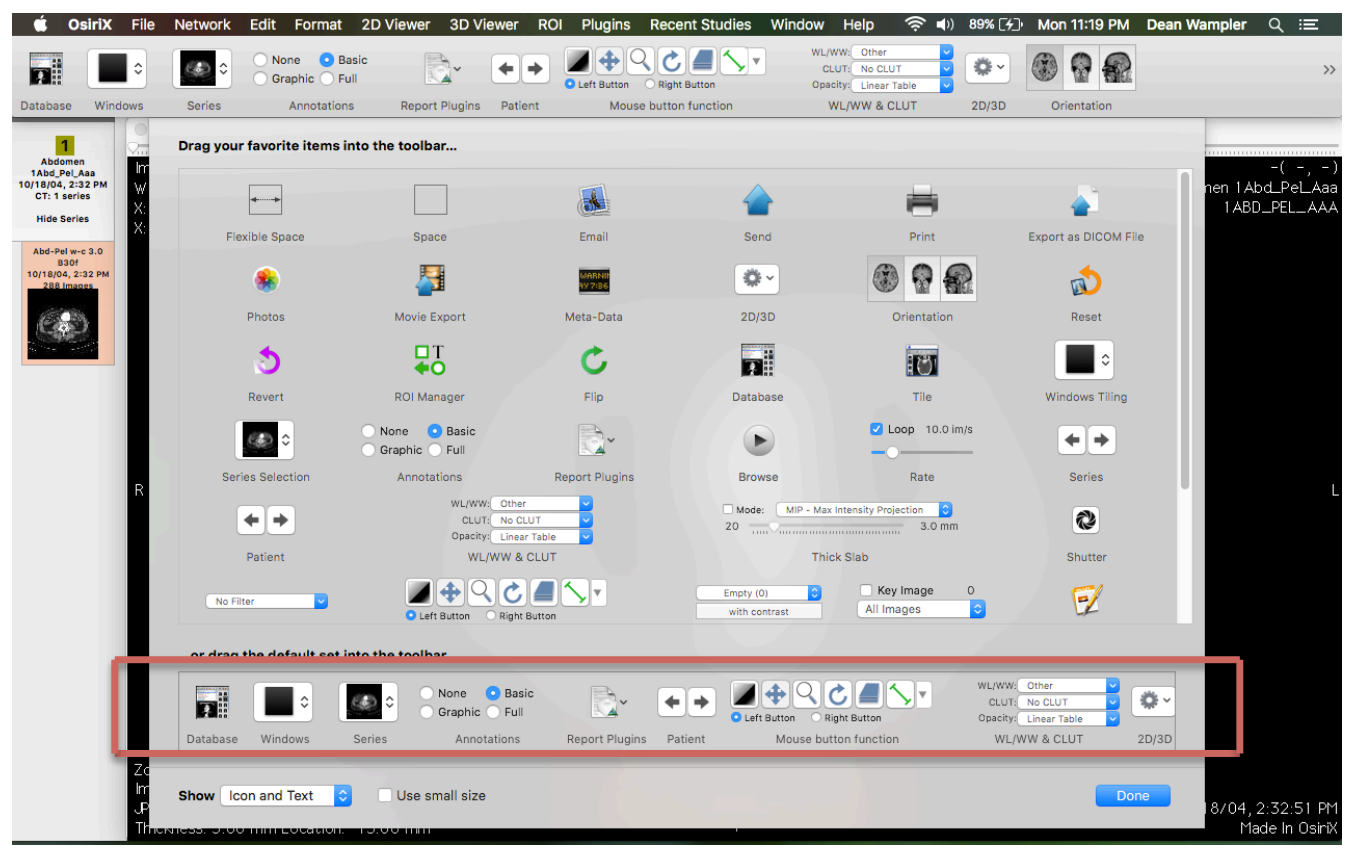


3. Select group of images to adjust window threshold level. Select the icon and move the mouse up and down to find the optimal range

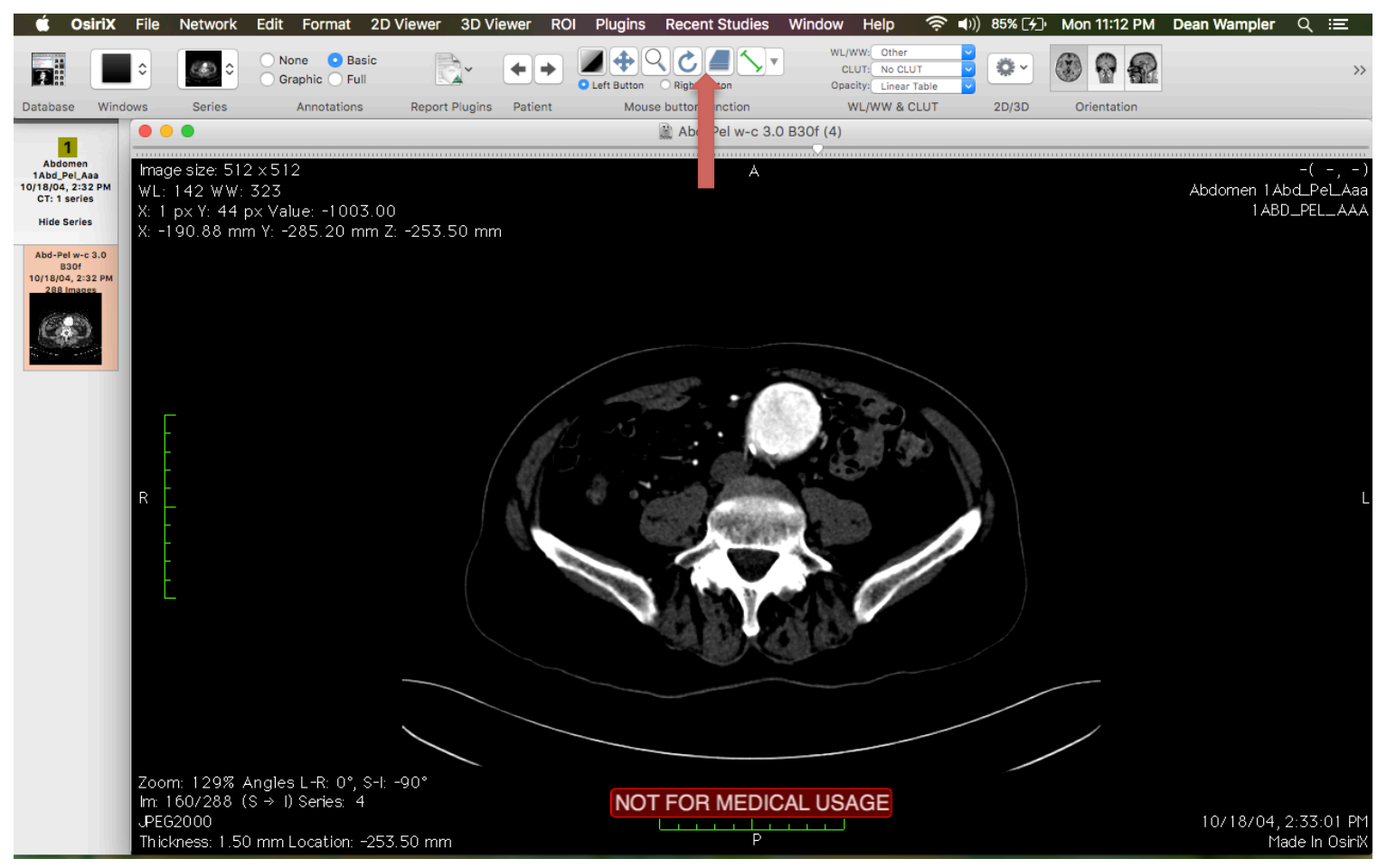


4. Select the bone using the ROI tool so that we can remove it from the data.

a. $\quad$ ROI $>$ Grow Region (2D/3D Segmentation)...

b. Select "3D growing region"

c. Select for algorithm "Threshold lower/upper bounds"

d. Lower threshold: 200

e. Upper threshold: 3000

f. Select the section of bone from the images by clicking the bone, then clicking compute

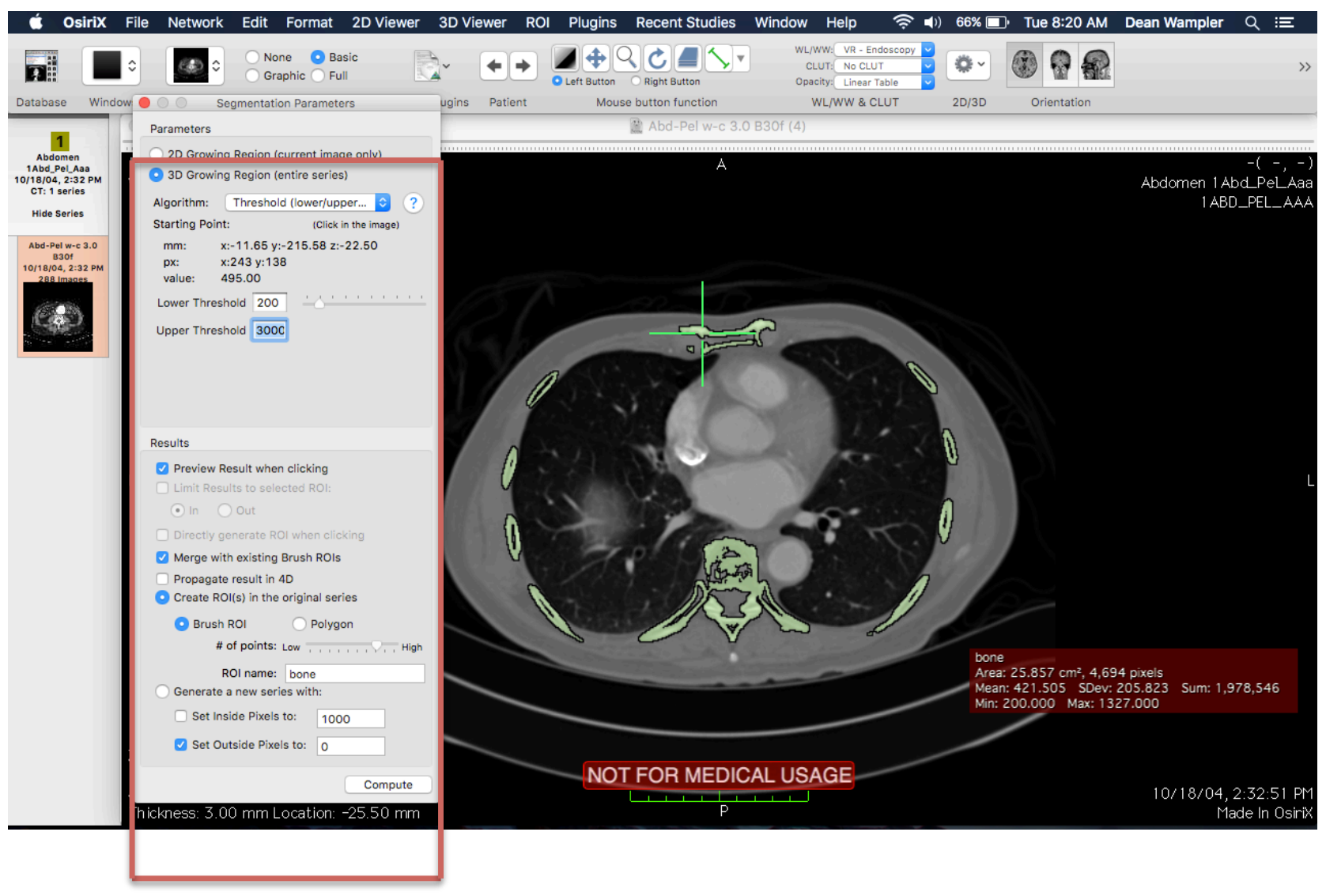


5. Fill and expand selection. Fill with black pixels, to make it disappear

a. ROI $>$ Brush ROI $>$ Dilation

i. Set Structuring Element Radius to 5 pixels

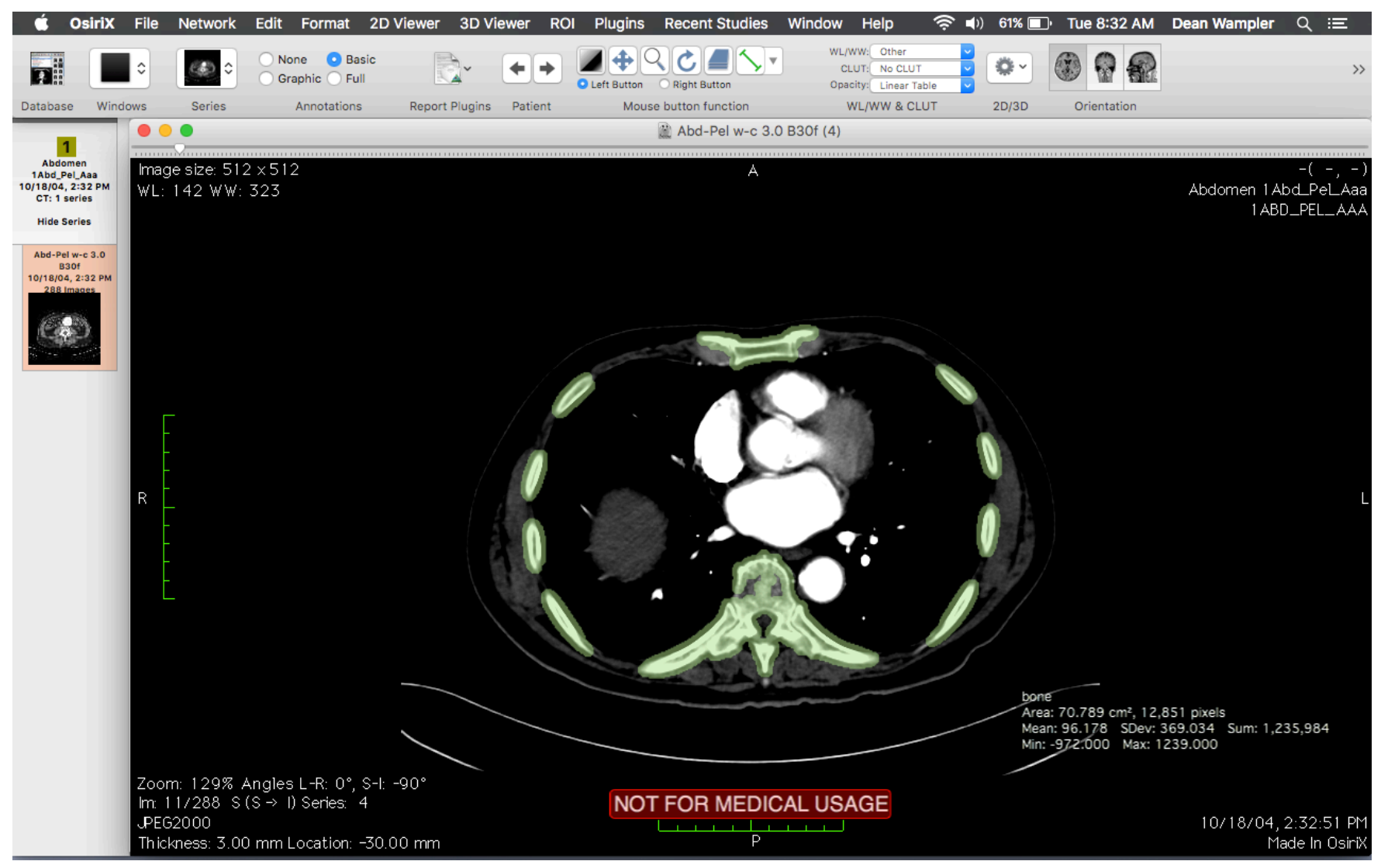


6. Fill in the selected ROI so that the pixel values can be set to black, making the bone ROI disappear

a. $\quad$ ROI $>$ Set pixel values to...

b. Set all pixels inside the selected ROI to -3000 (black)

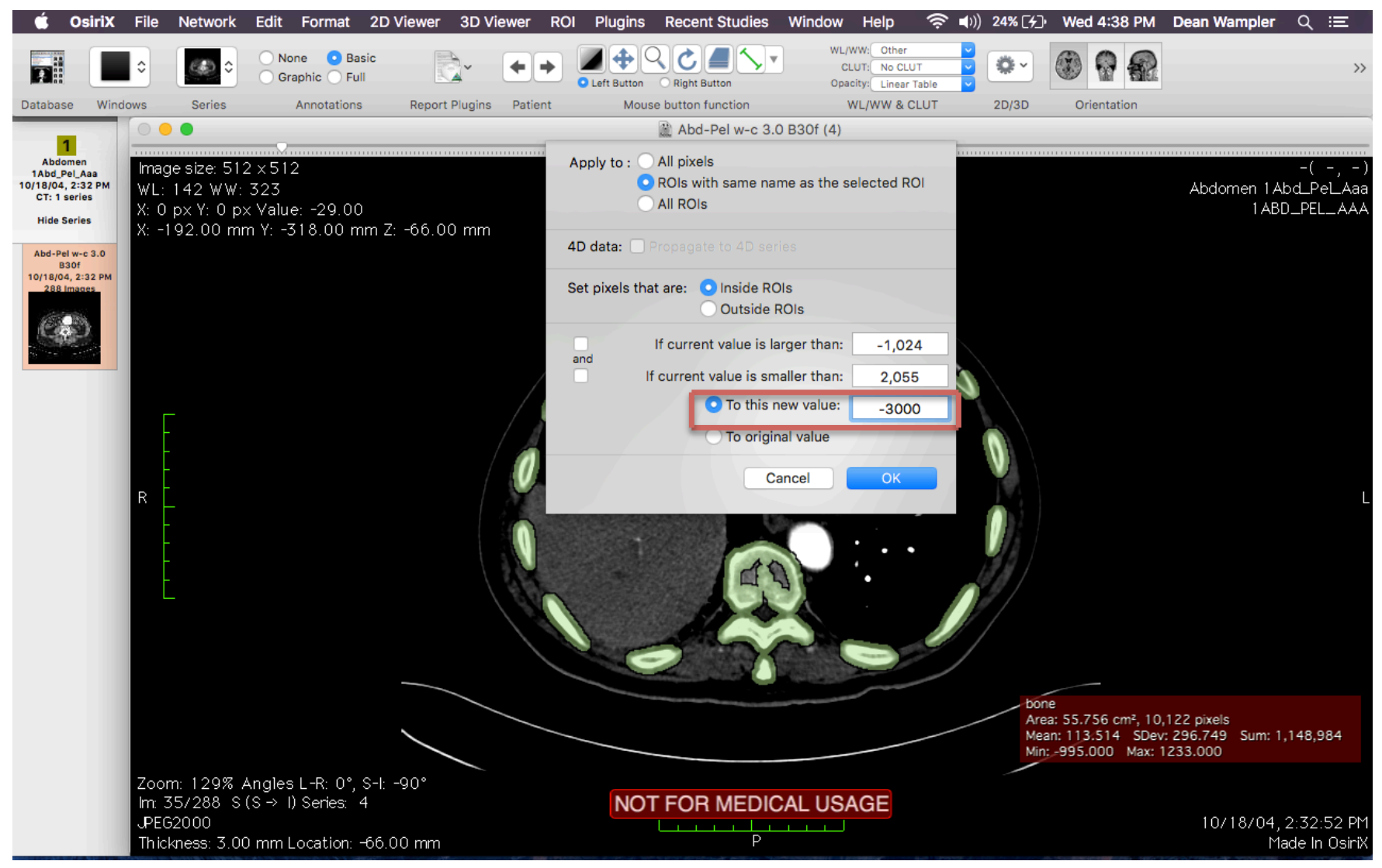


7. Adjust the images so that the $3 \mathrm{D}$ rendering forms an enclosed surface.

a. Create a rectangle that covers the entire image on the first and last image of the series.

b. Set the pixel values to -3000 using the technique previously described (ROI > set pixel values to...)

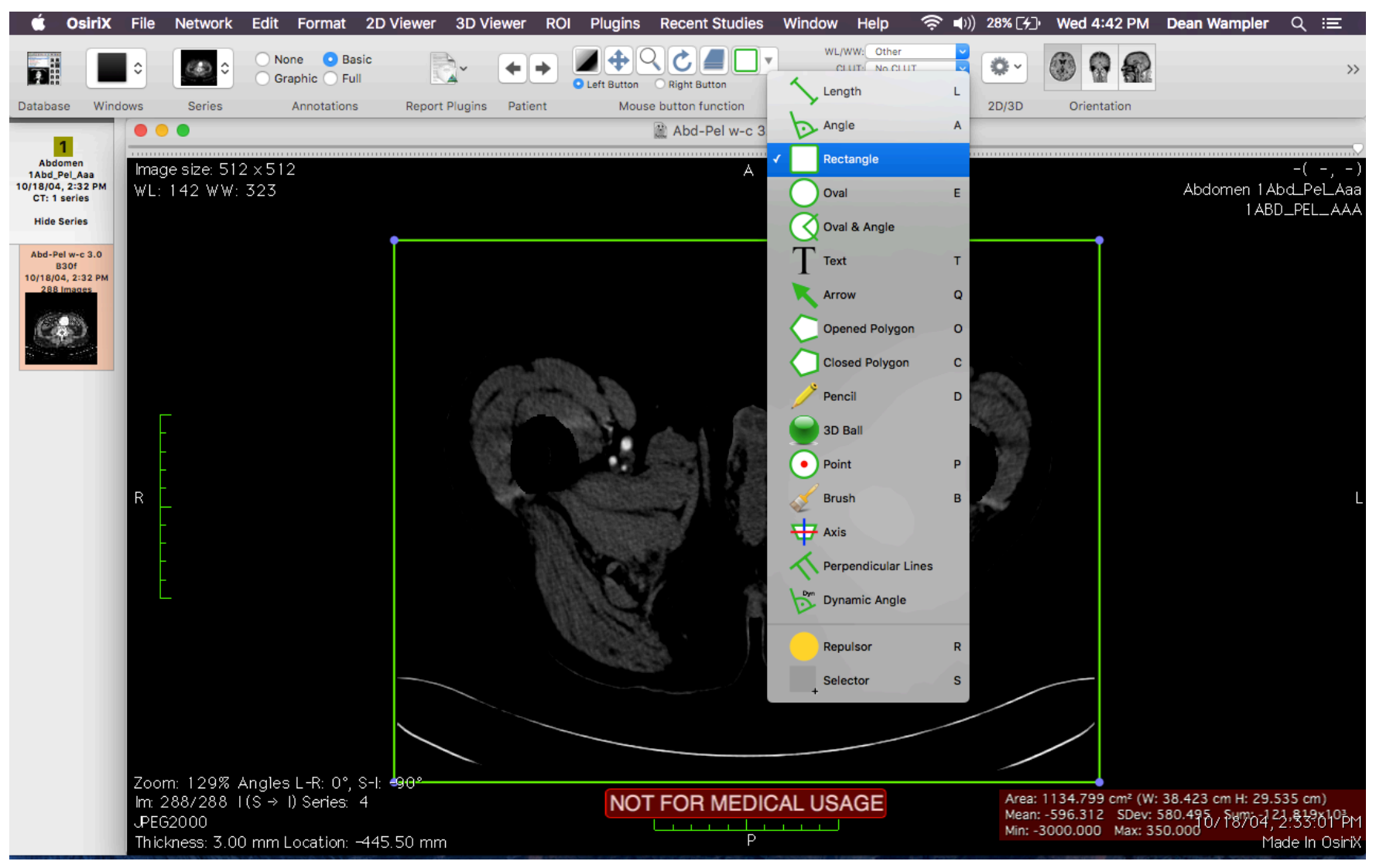


8. Create new ROI segmentation for vessels
a. ROI $>$ Grow Region (2D/3D Segmentation)...
b. Set lower threshold to 200
c. Set upper threshold to 1000
d. Rename ROI to "vessels"
e. Compute

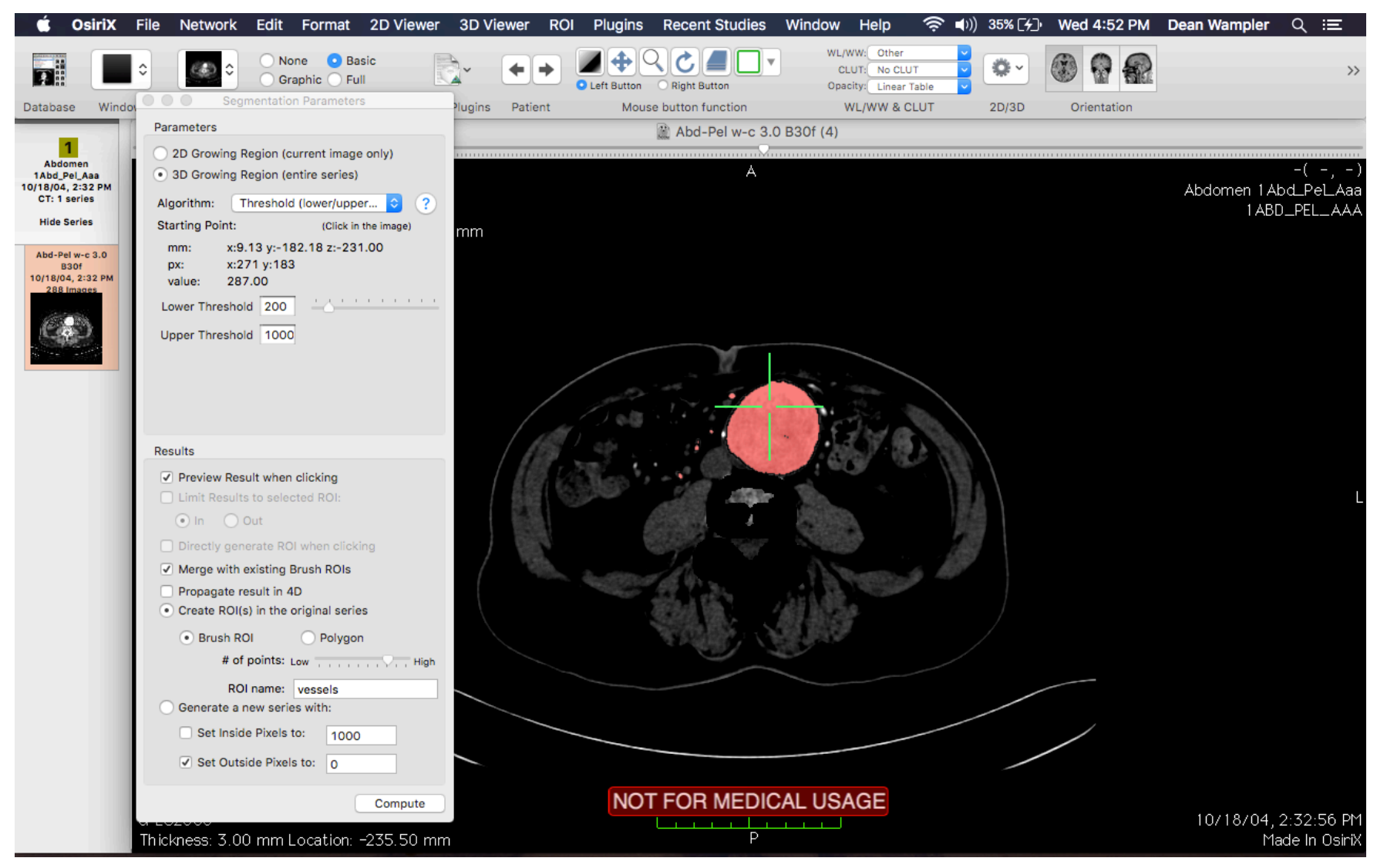


9. Set all pixel values outside the selected ROI to black

a. $\quad$ ROI $>$ Set pixel values to...

b. Set pixels that are: Outside ROI to -3000 (black)

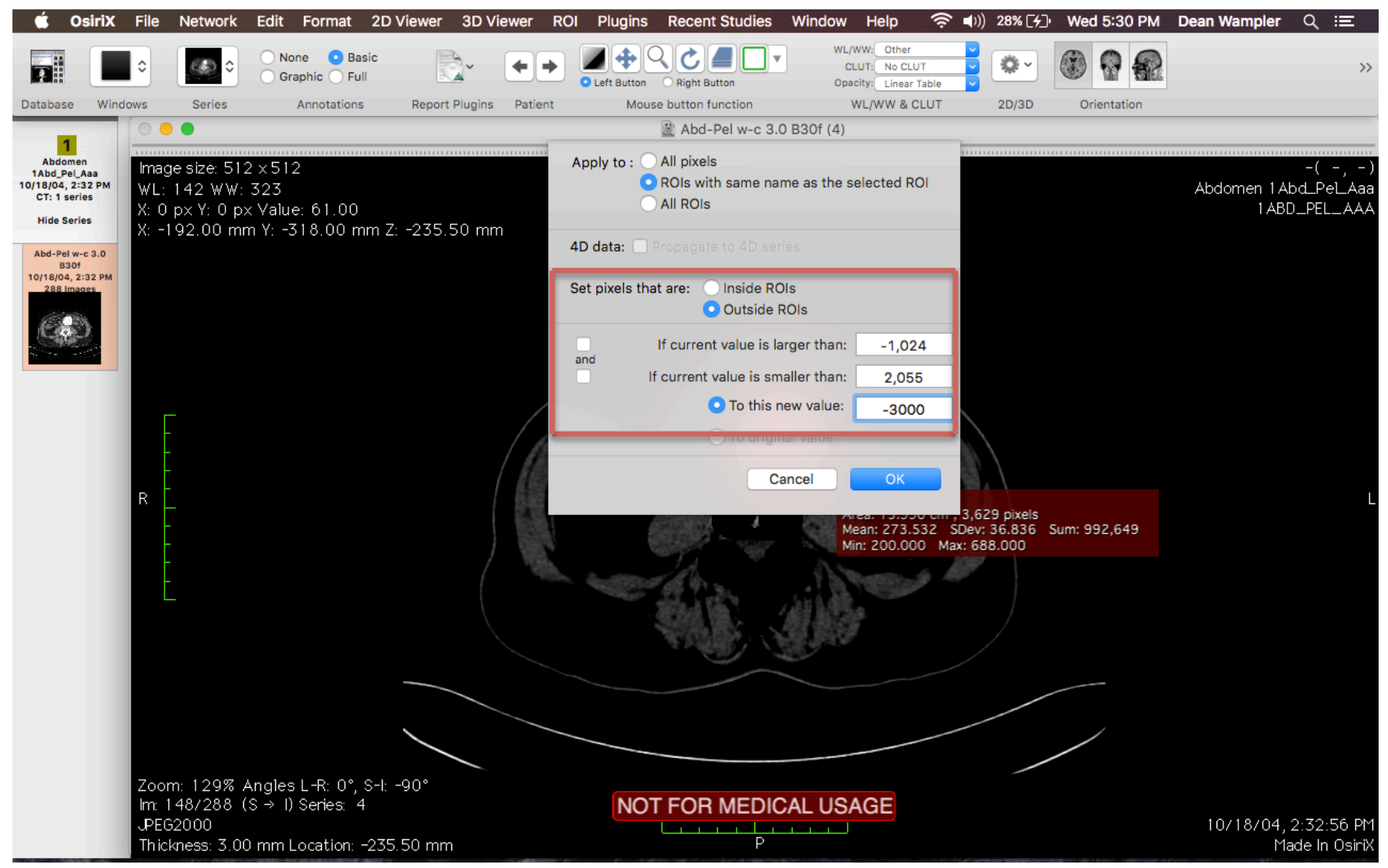


10. Set all pixel values outside the selected ROI to white

a. $\quad$ ROI $>$ Set pixel values to...

b. Set pixels that are: Outside ROI to 3000 (white)

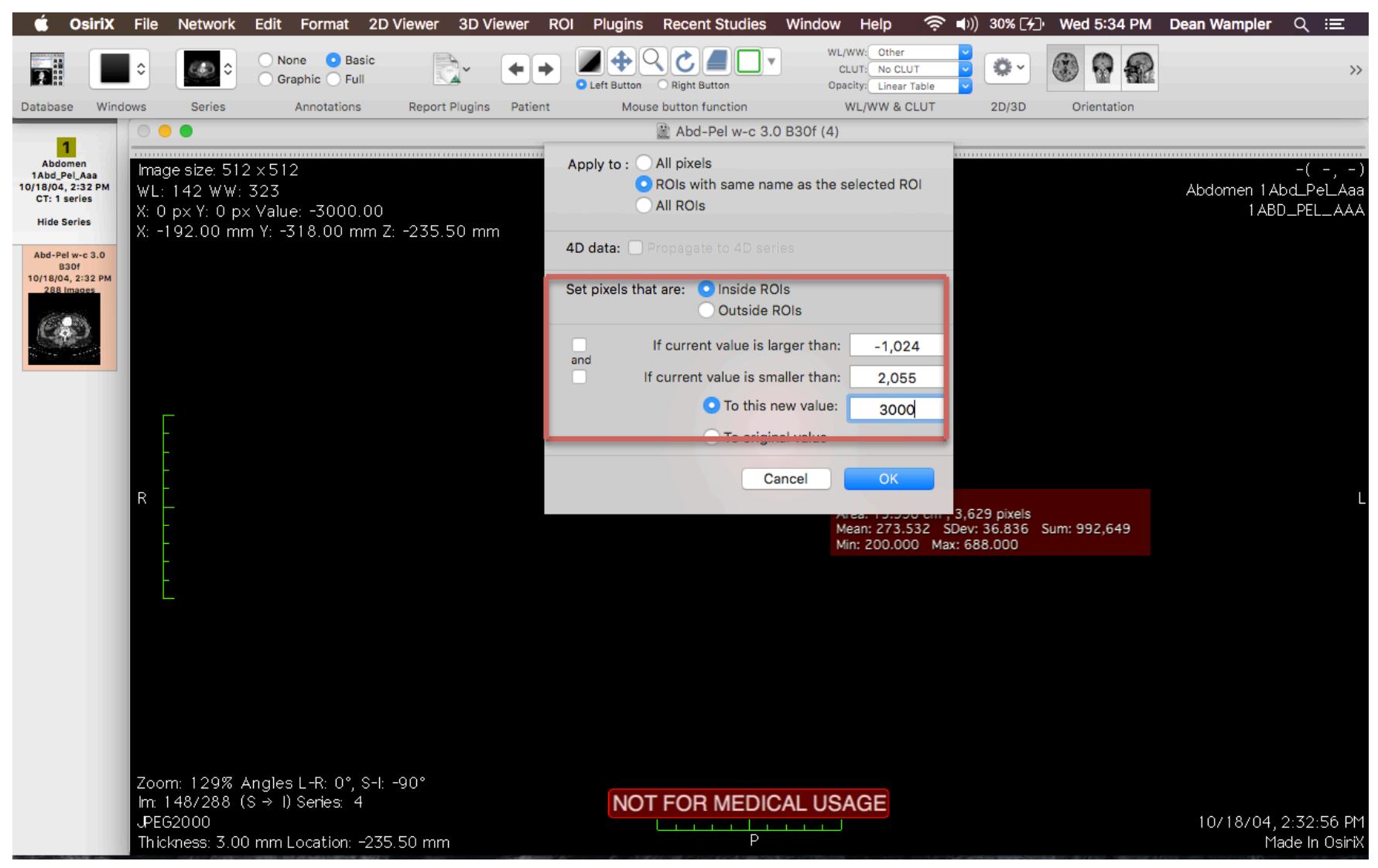


11. The 3D surface rendering should contain the isolated AAA information

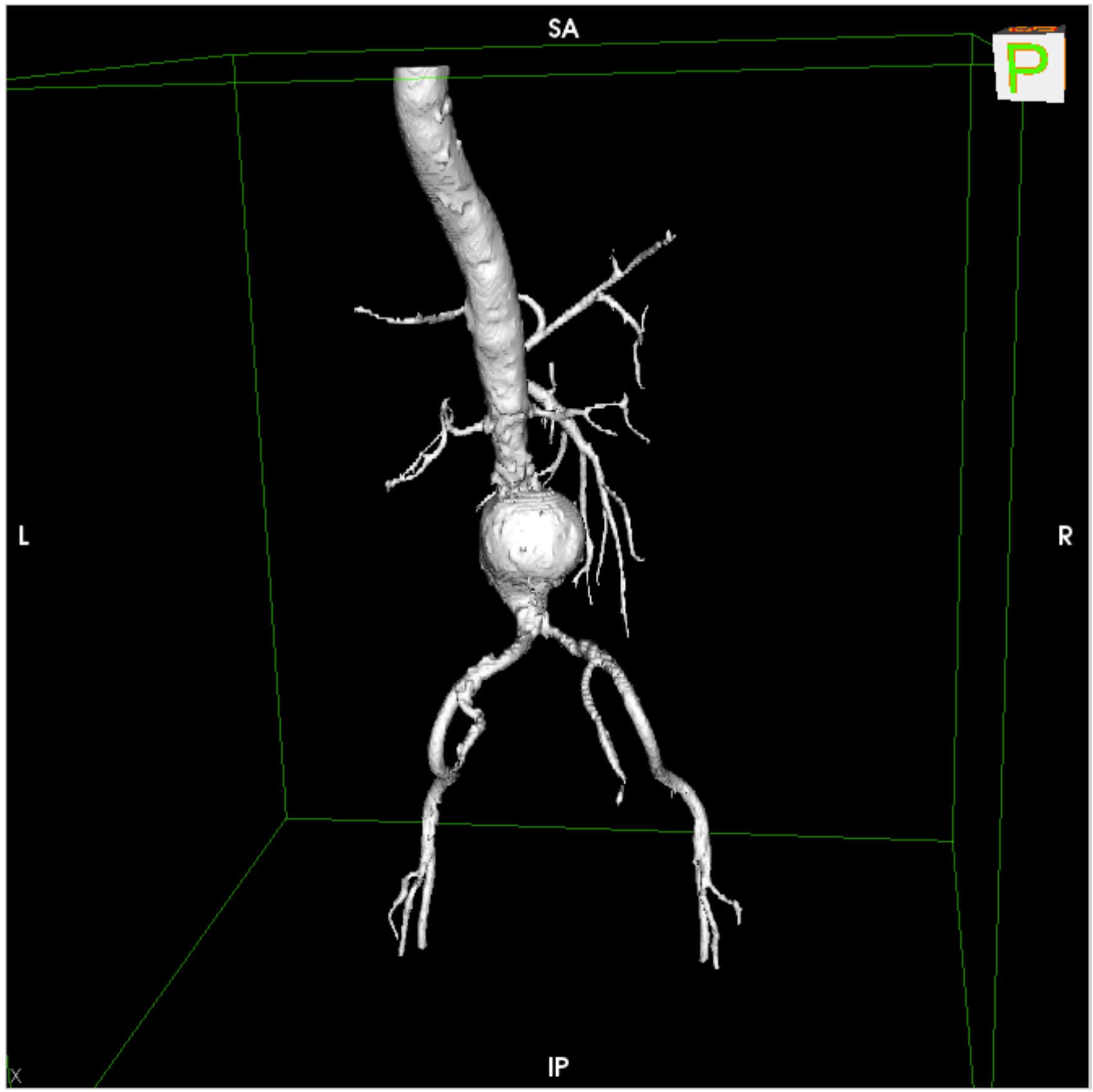


12. Remove all arteries/vessels that are not necessary for flow analysis. I will only leave the renal arteries. I will also remove the iliac arteries, leaving only the section that comes directly before.

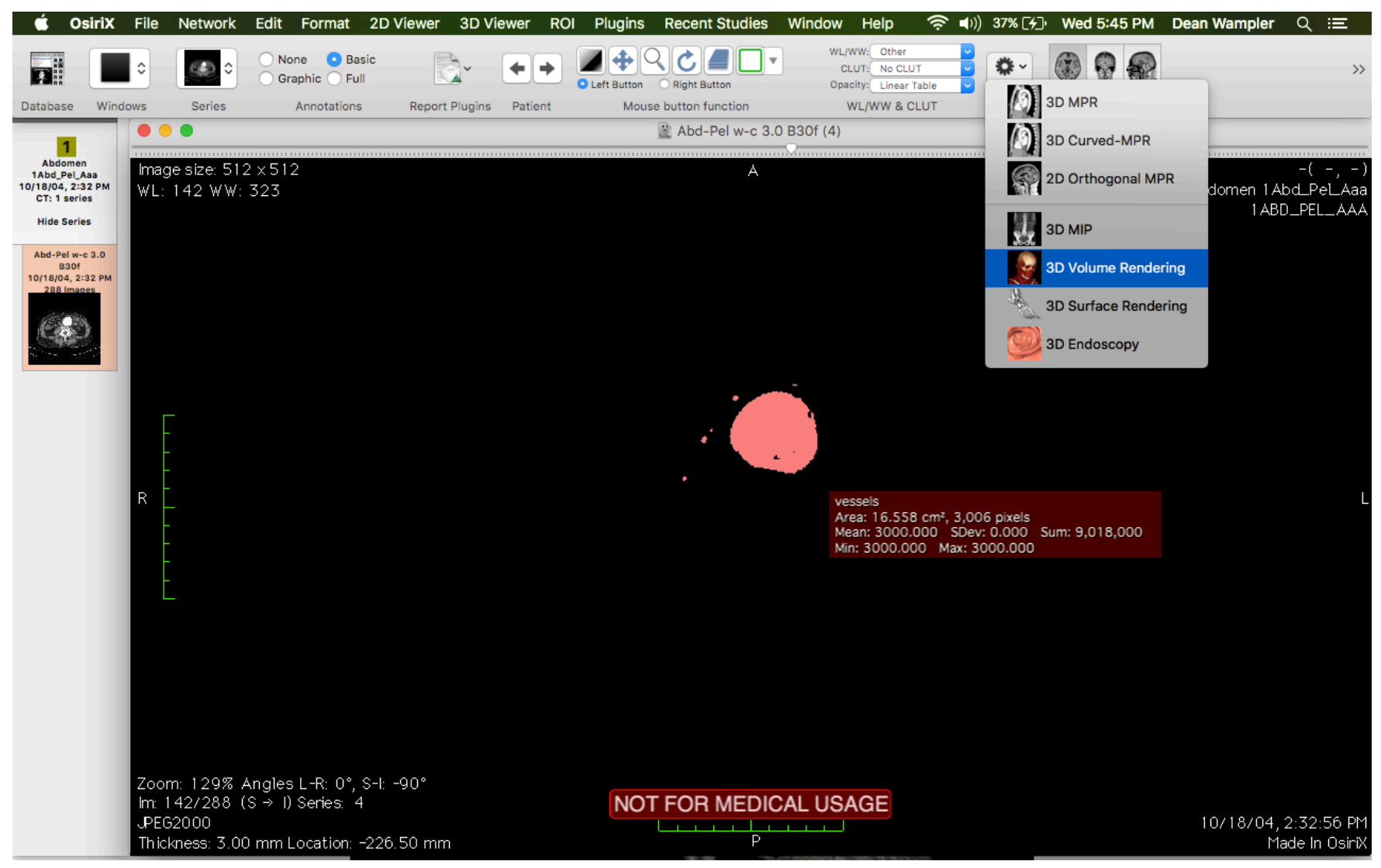


13. The final volume rendering after removing the appropriate arteries should contain the desired AAA information

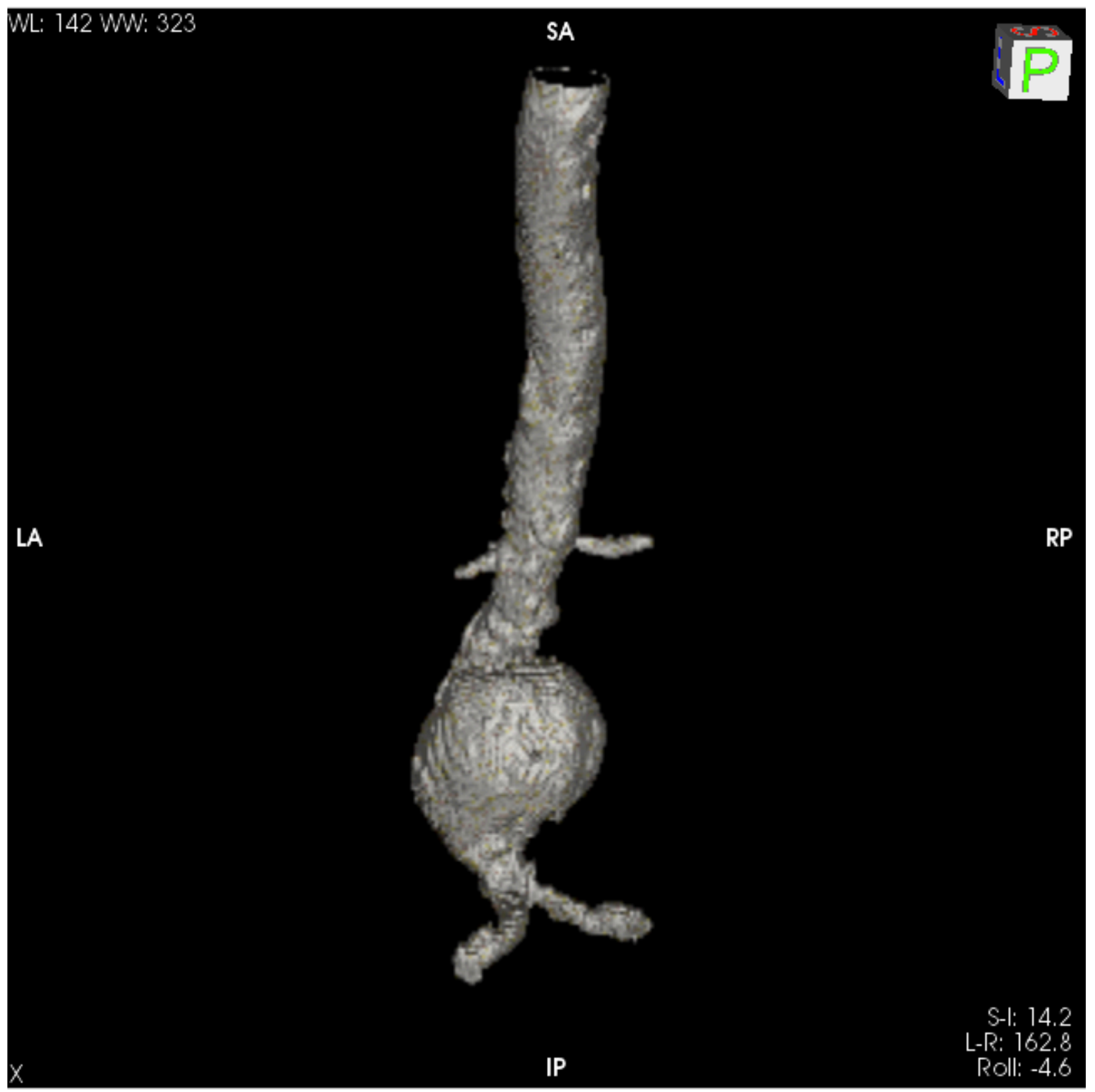


14. In volume rendering, export the file to a .STL file format to be adjusted in

Meshmixer

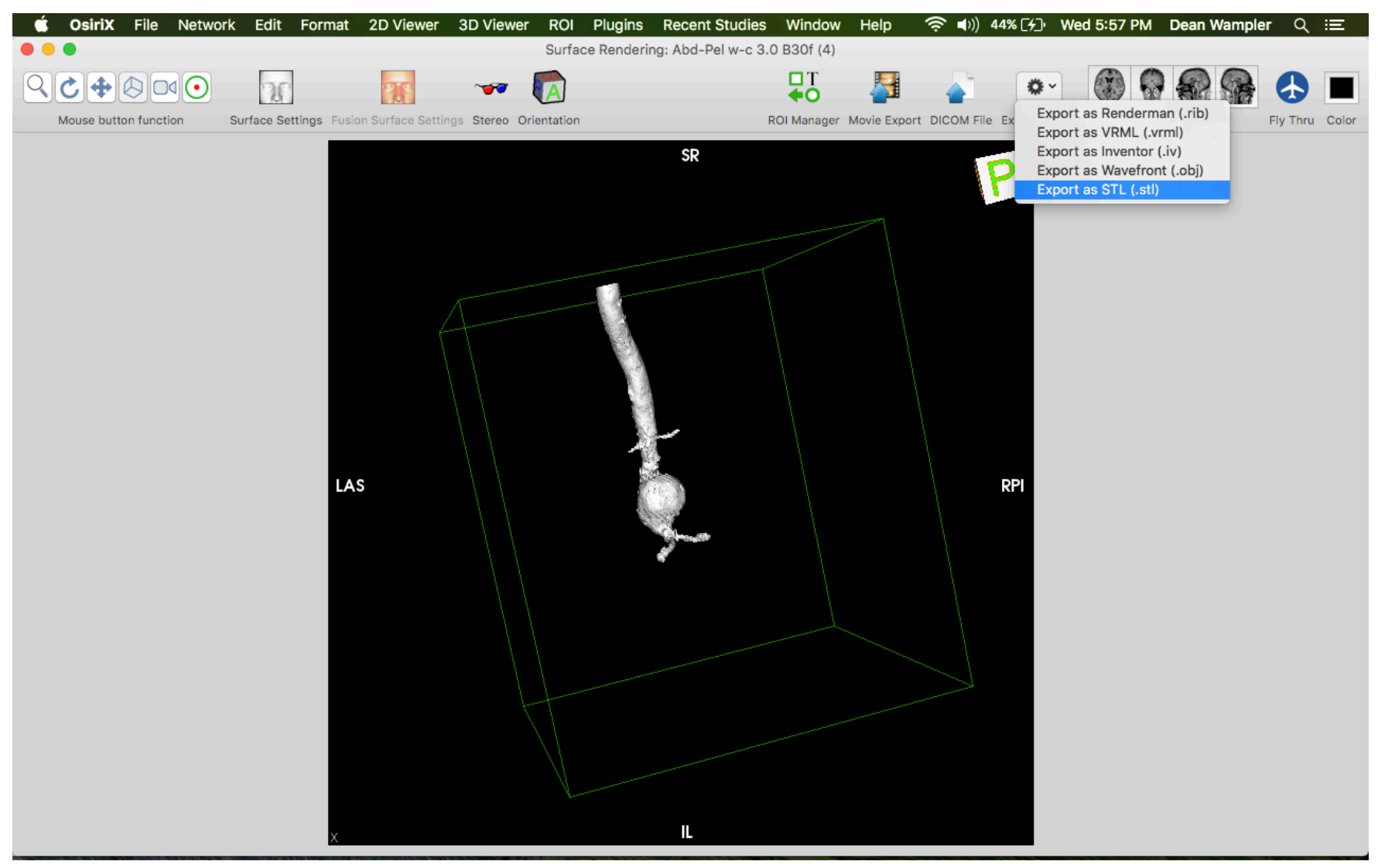




\section{Appendix B: STL to Solid Body}

1. Import .STL file into Autodesk Meshmixer

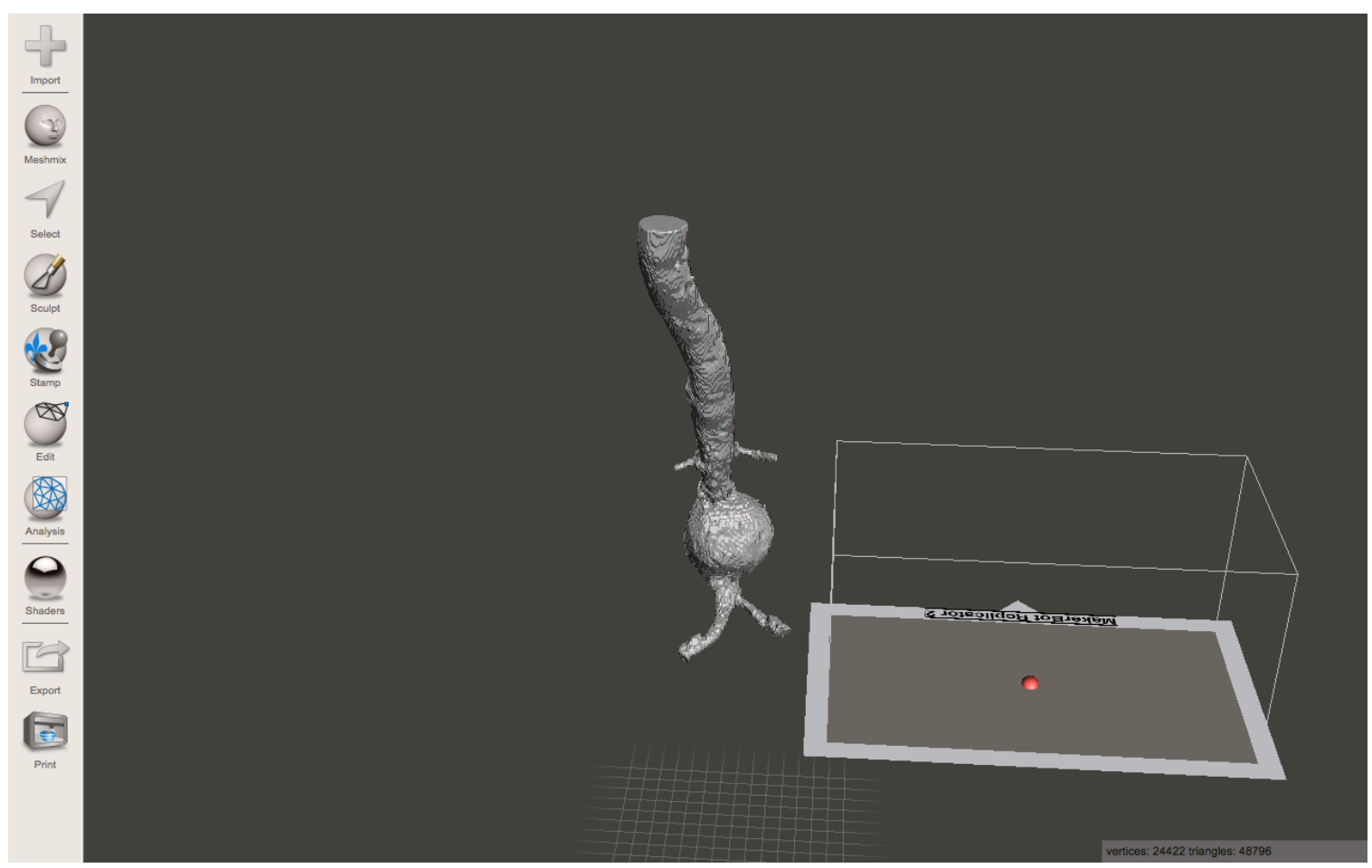


2. Make the object a solid
a. $\quad$ Edit $>$ Make Solid
b. Solid type: Accurate
c. Solid Accuracy and Mesh Density to the maximum
d. Accept

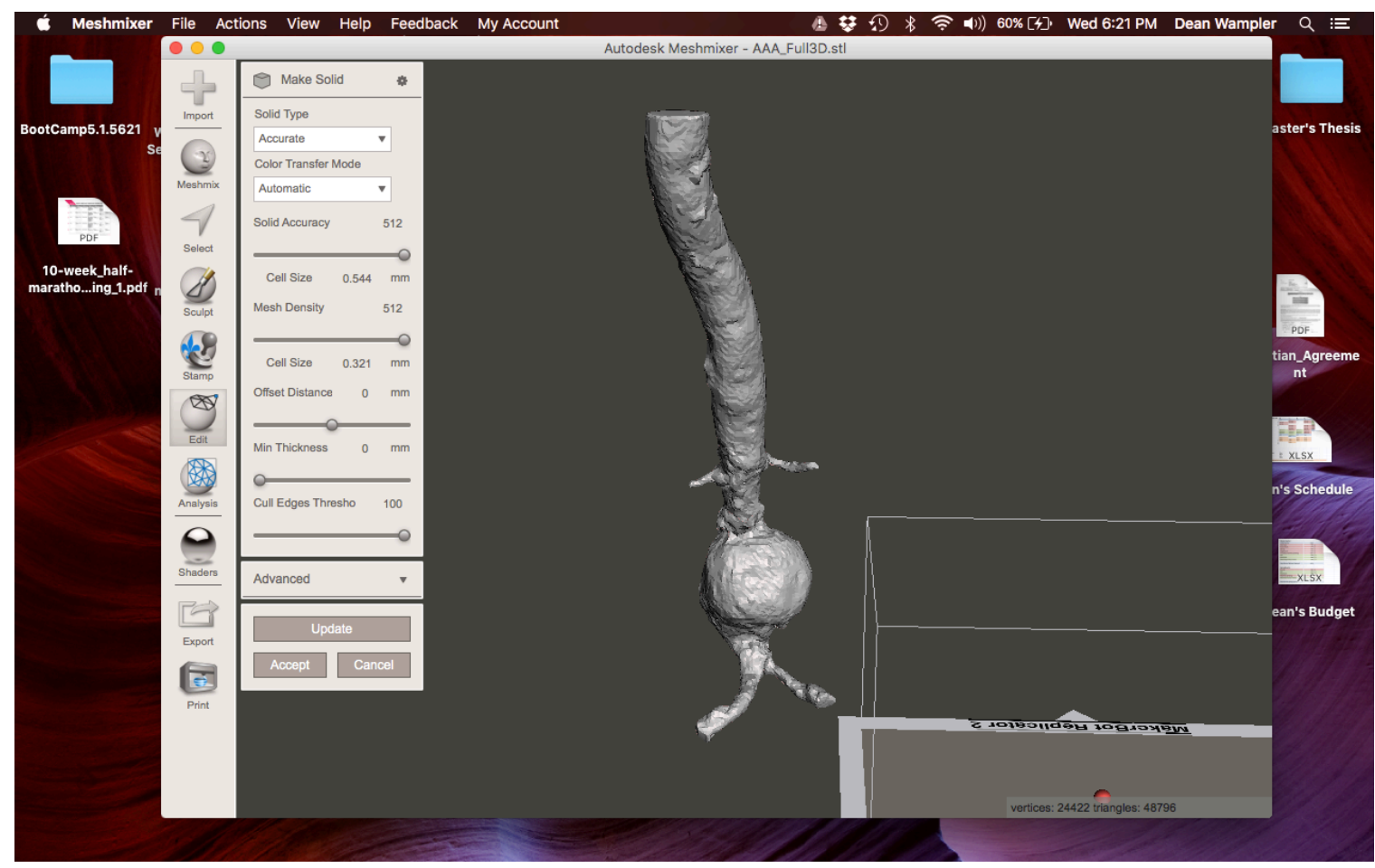


3. Select the entire object and smooth the edges

a. Select $>$ Deform $>$ Smooth

b. Smooth type: Cotan

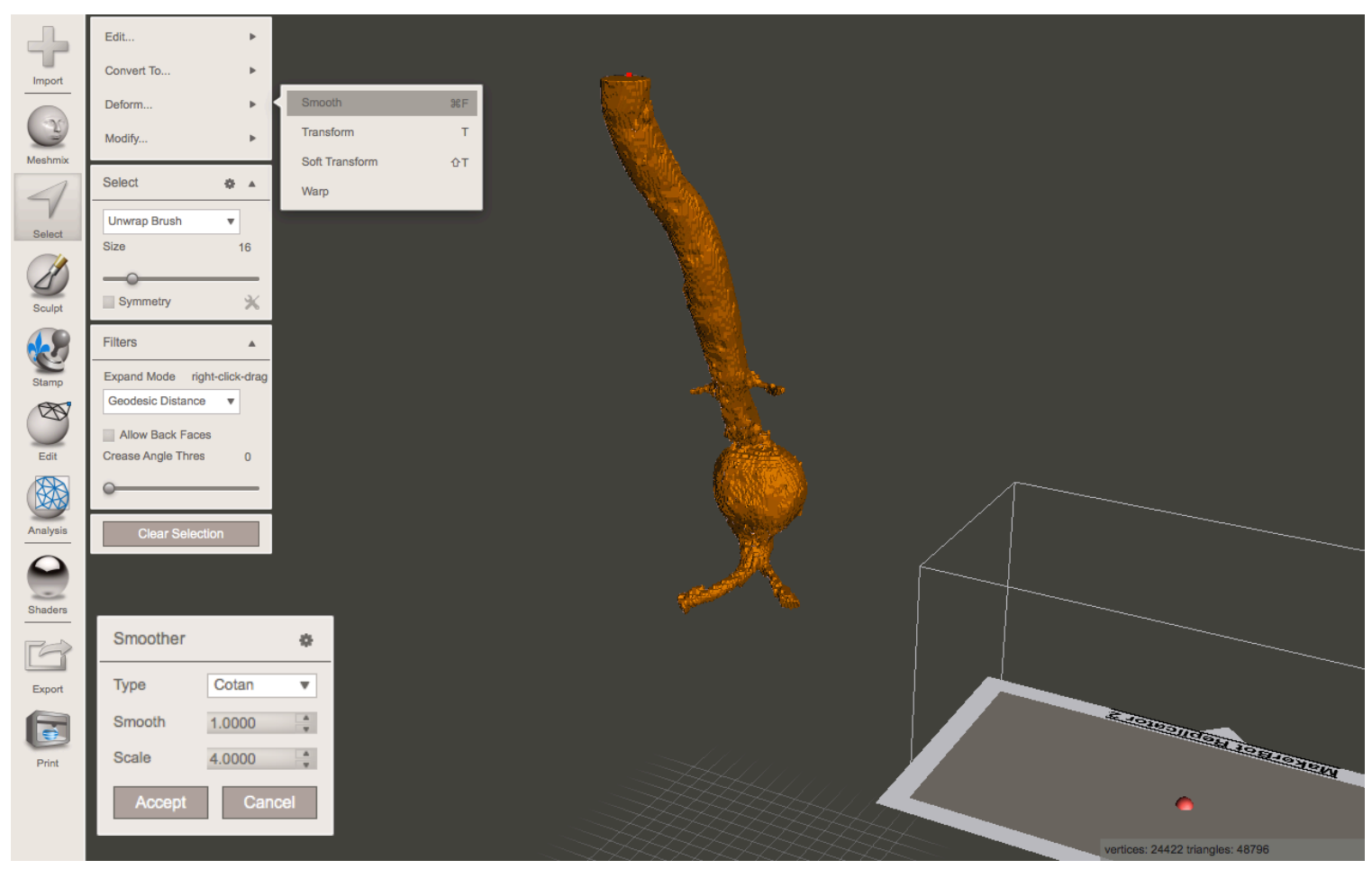


4. Remove unnecessary features such as bumps to make the file smoother.

a. $\quad$ Select $>$ Edit $>$ Erase and Fill

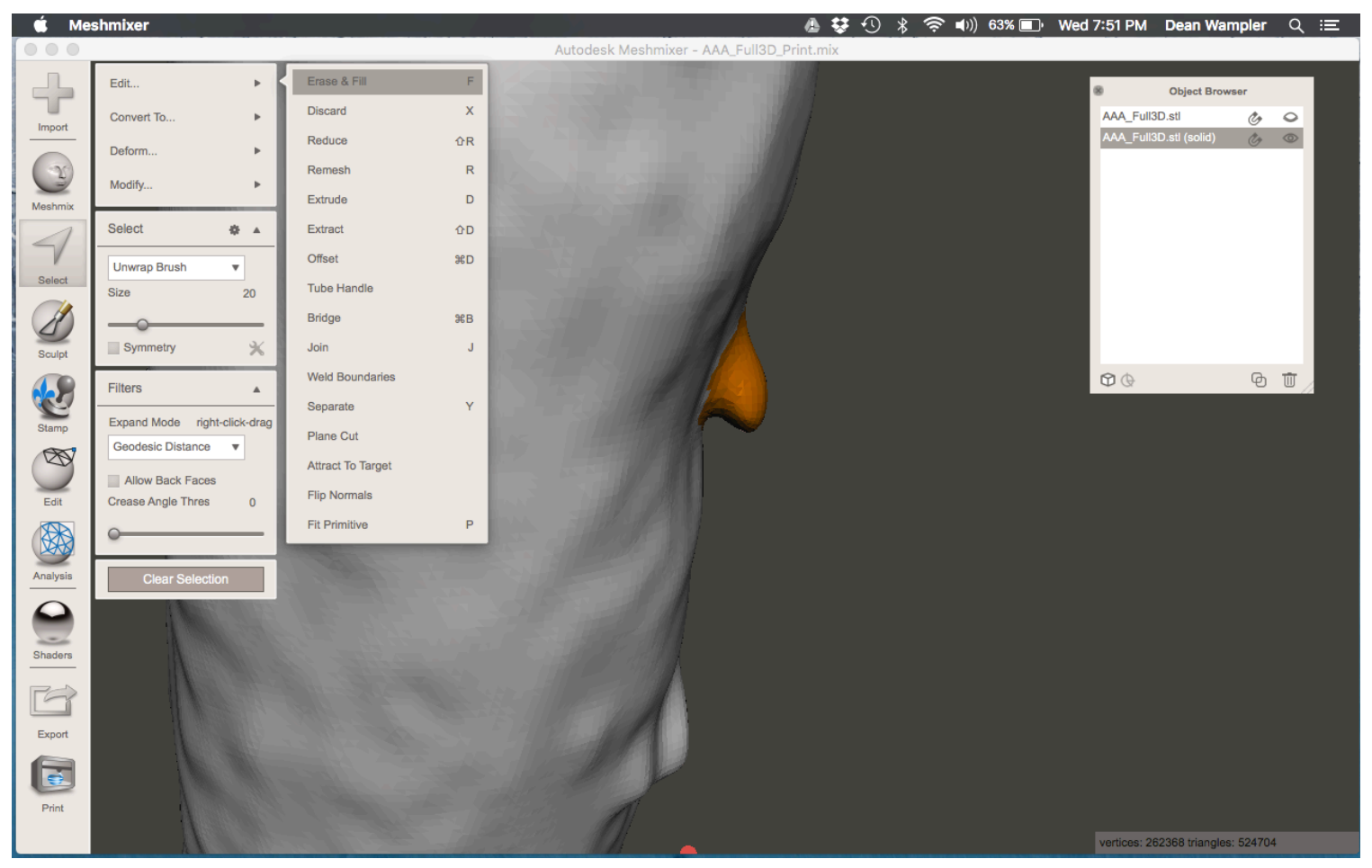


5. Select the whole object and smooth the surface again

a. Select $>$ Deform $>$ Smooth

b. Smooth type: Cotan

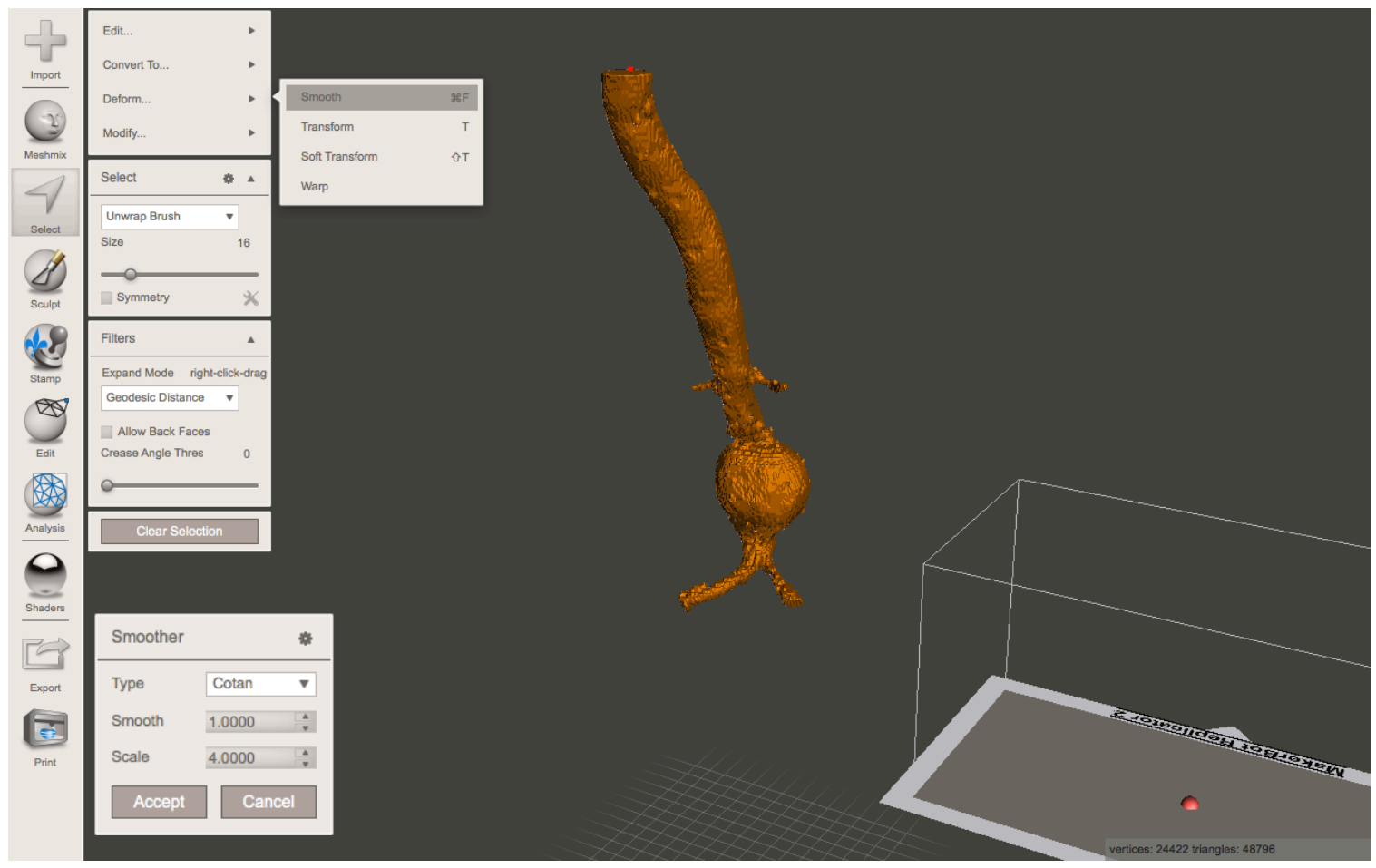


6. The final edited file should be a smoothed AAA model:

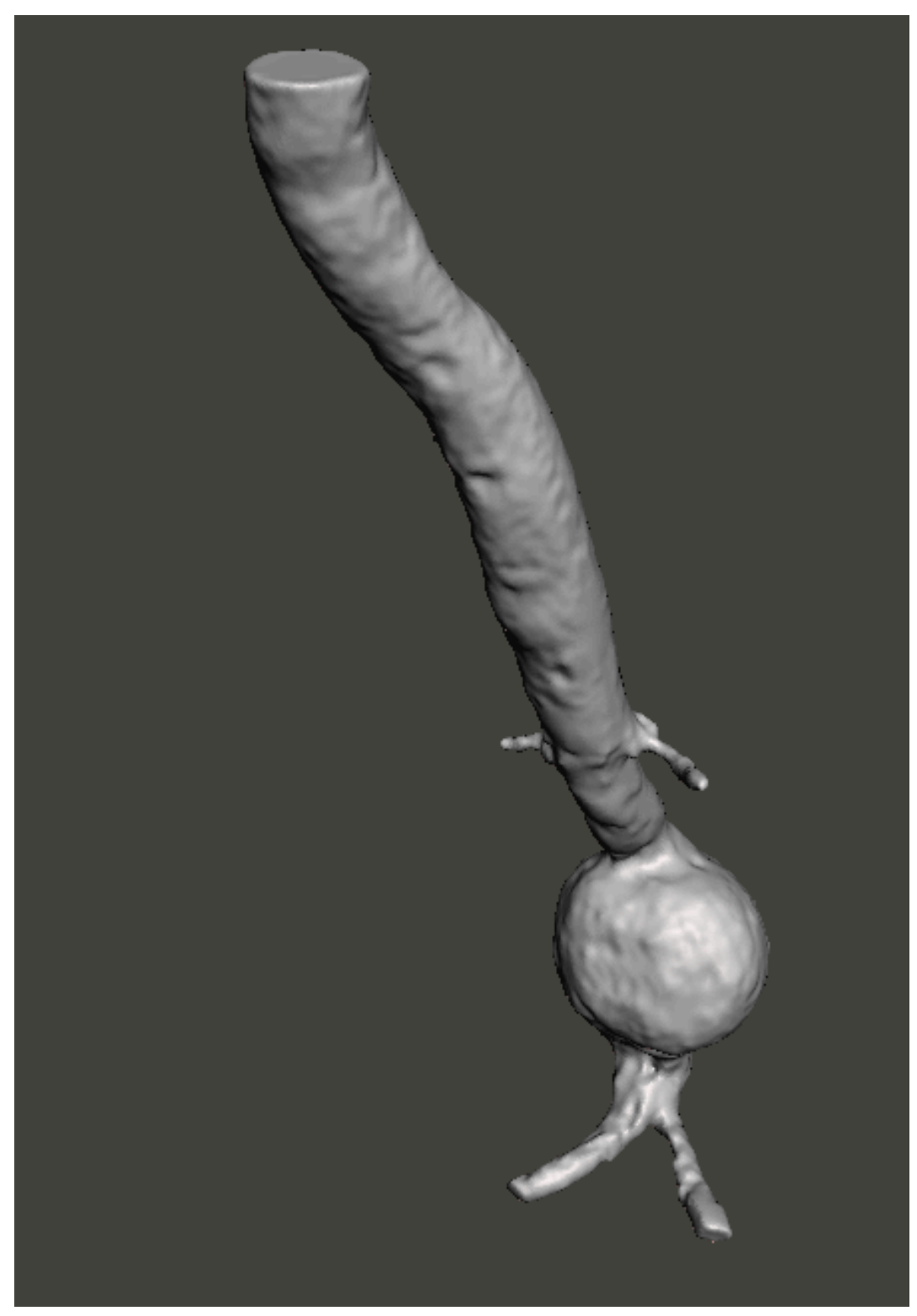


7. Make the object a solid again (this will get rid of overlapping faces)
a. $\quad$ Edit $>$ Make Solid
b. Solid type: Accurate
c. Solid Accuracy and Mesh Density to the maximum
d. Accept

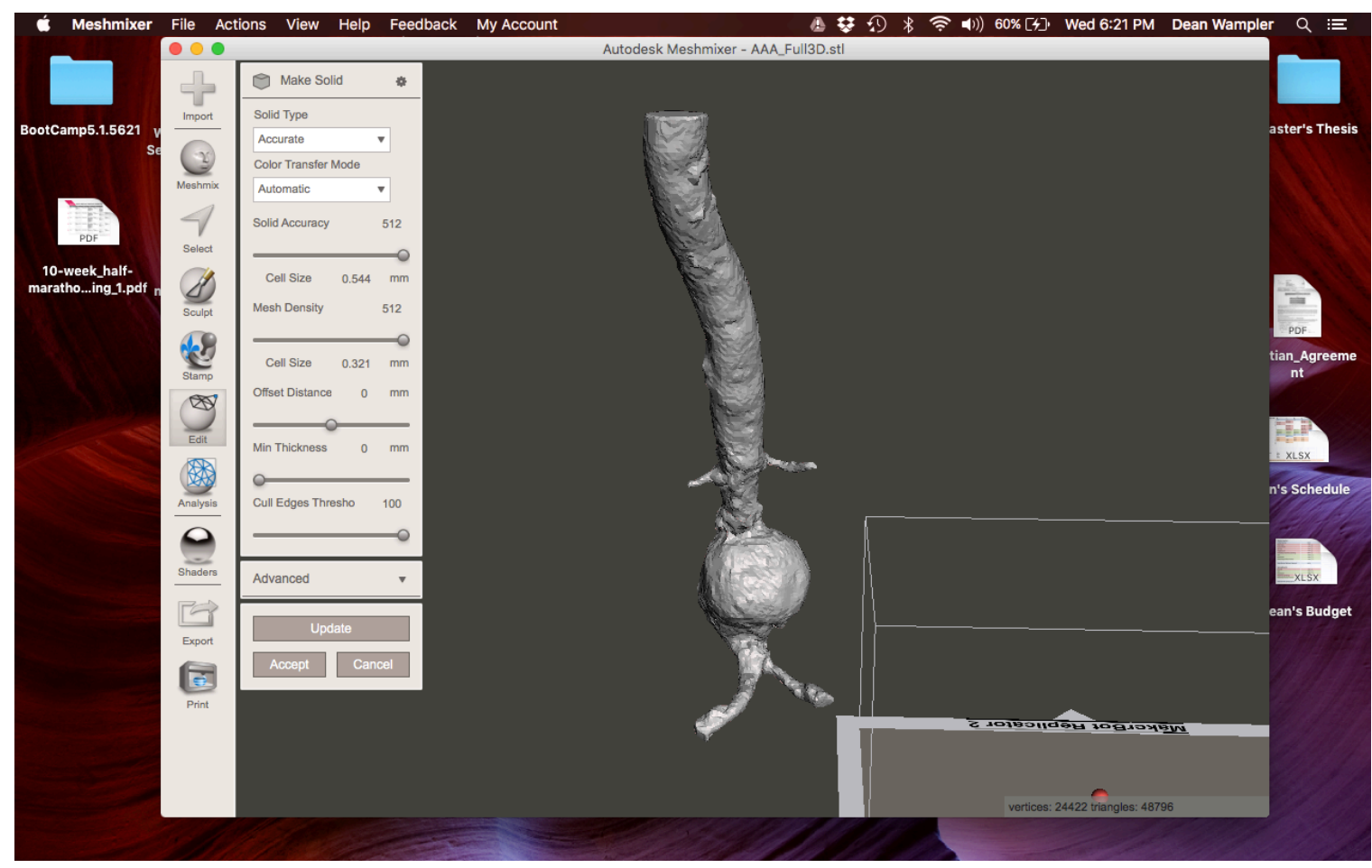


8. Export as a .STL binary file format

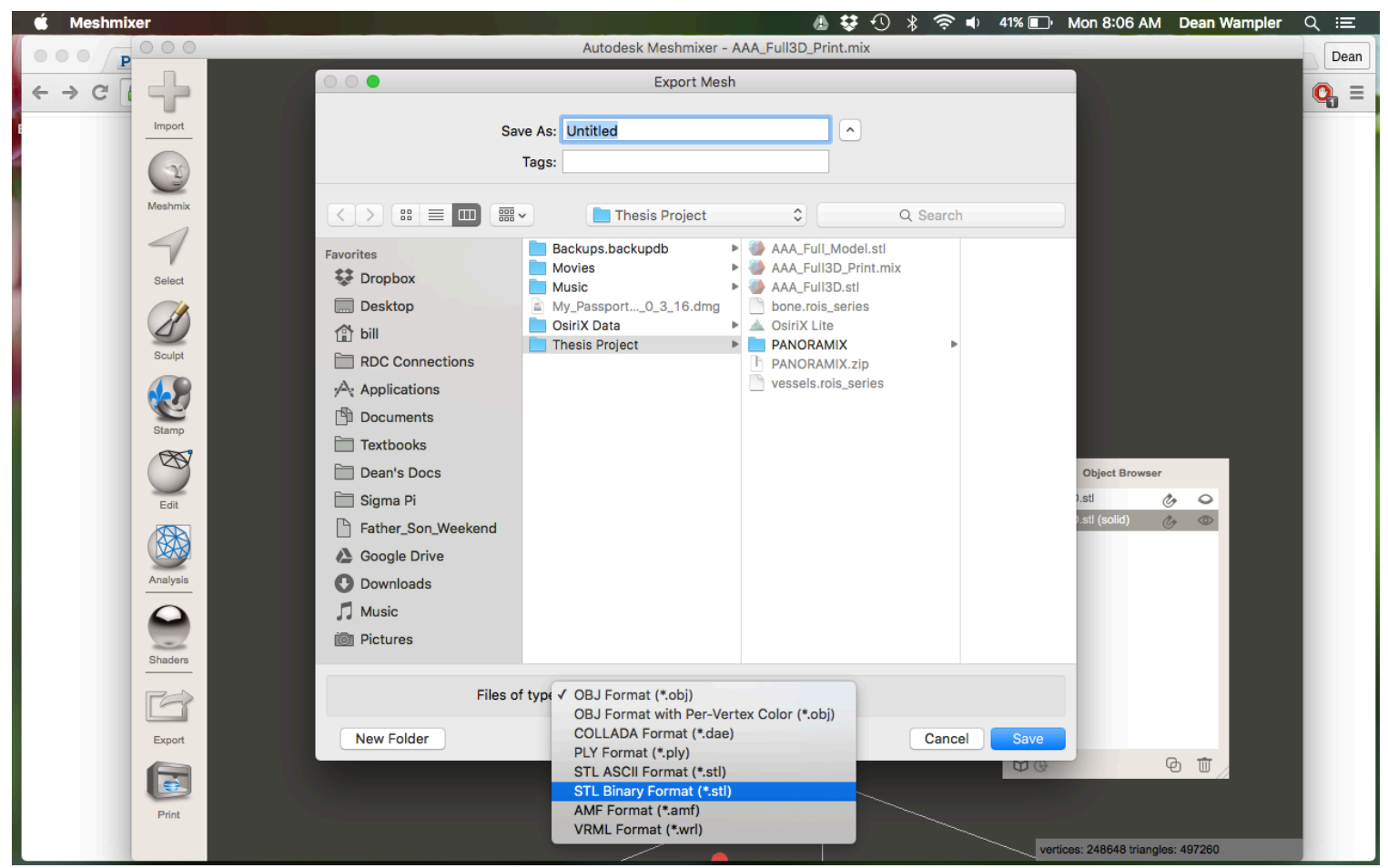




\section{Appendix C: Removing Unnecessary Aspects of AAA Model}

1. Open Solidworks.

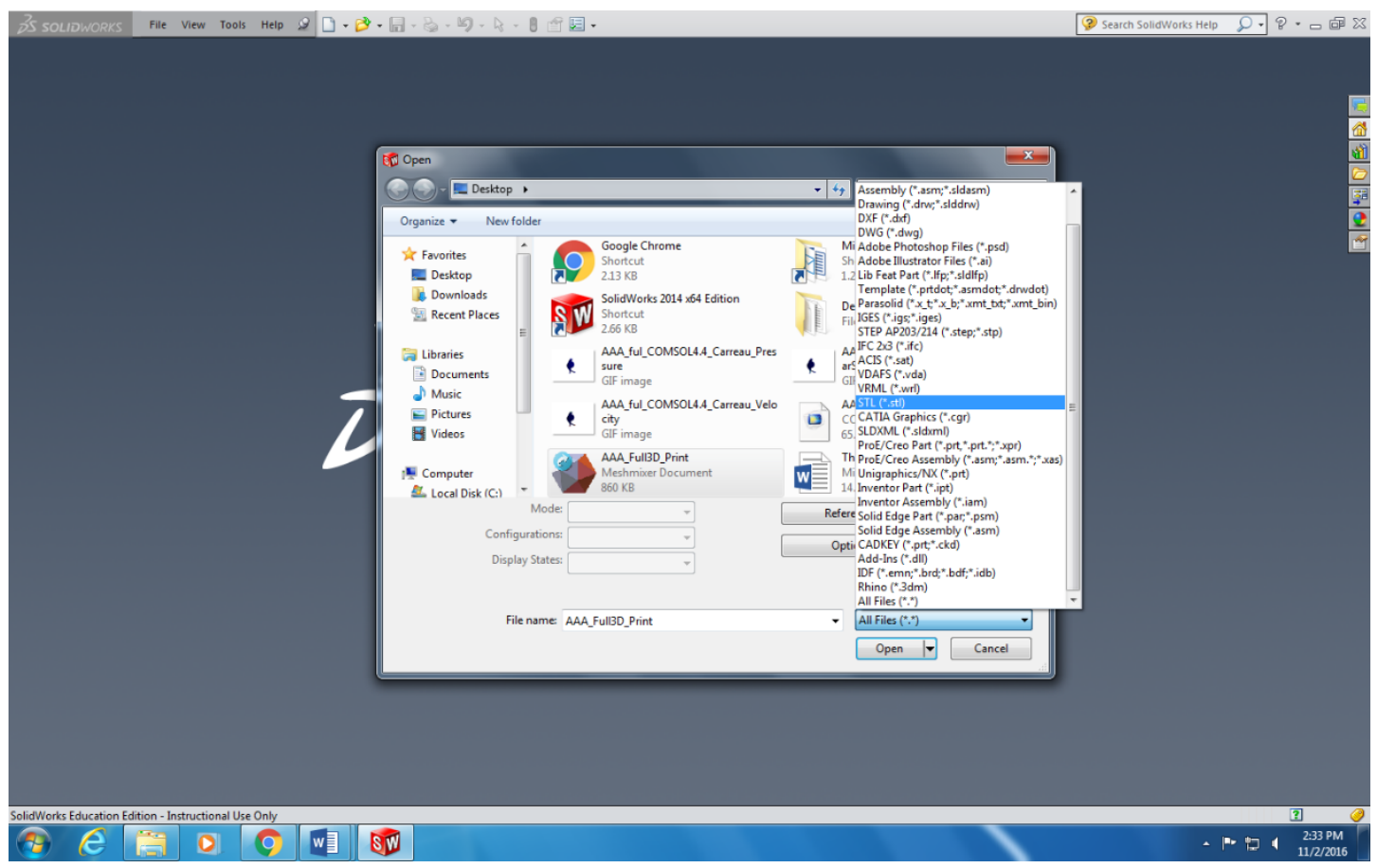


2. Import STL file. Select File type and choose STL files

3. Select options. Import File as a solid body. Chose the units to be centimeters. Select OK.

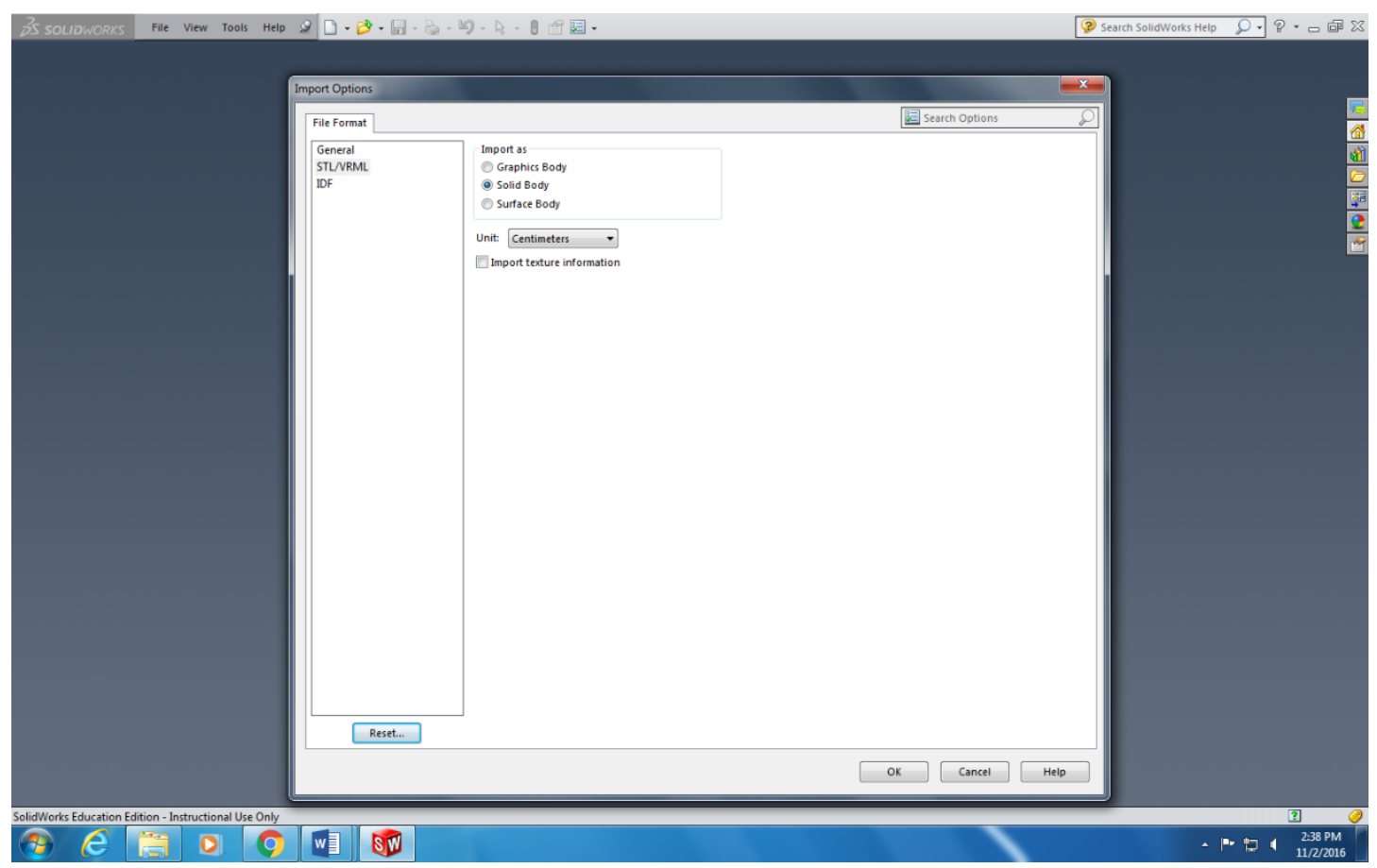

4. Open the STL file 
5. Run Import Diagnostics on the Part

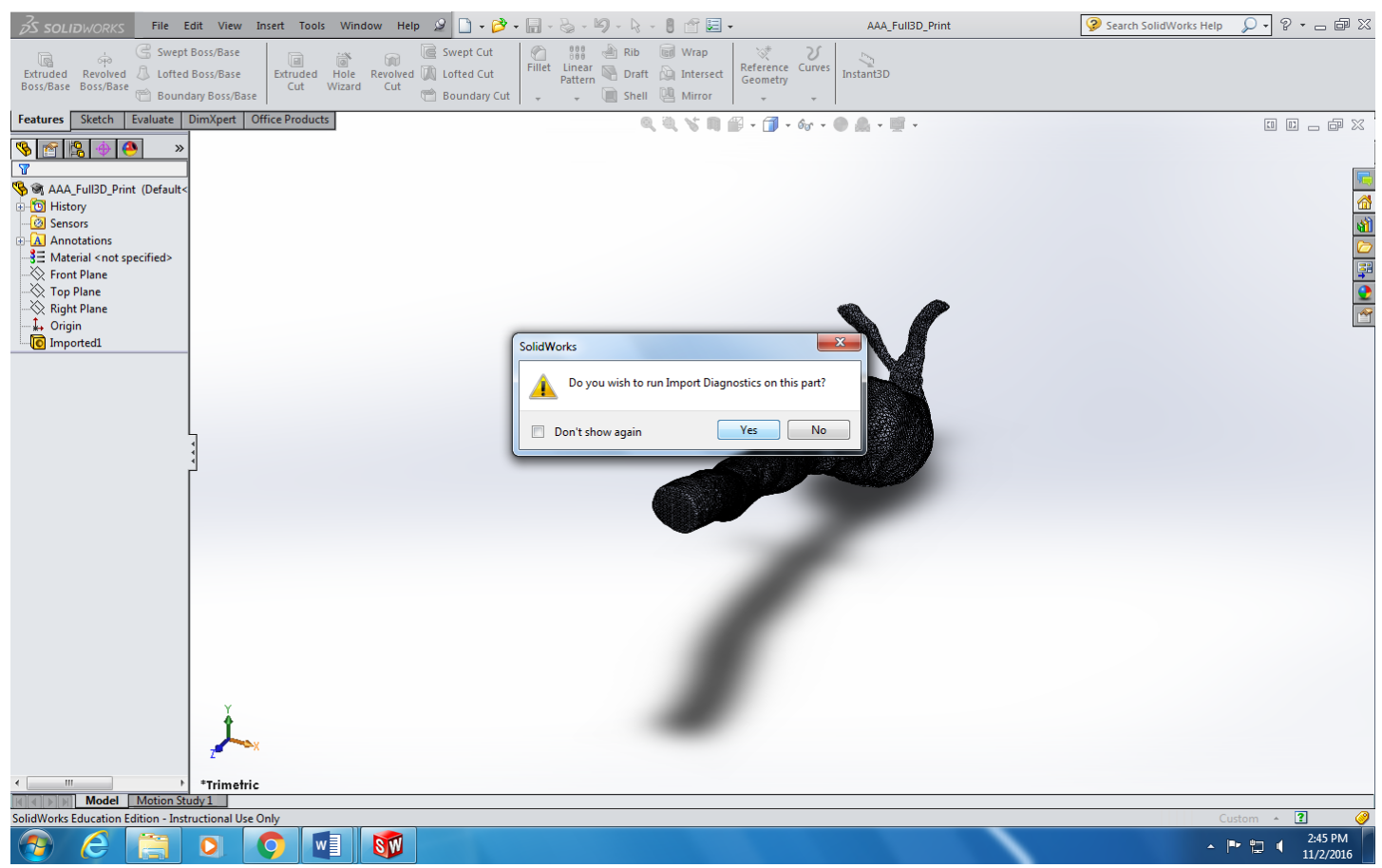


6. No faulty faces of gaps remain in the geometry. Select OK

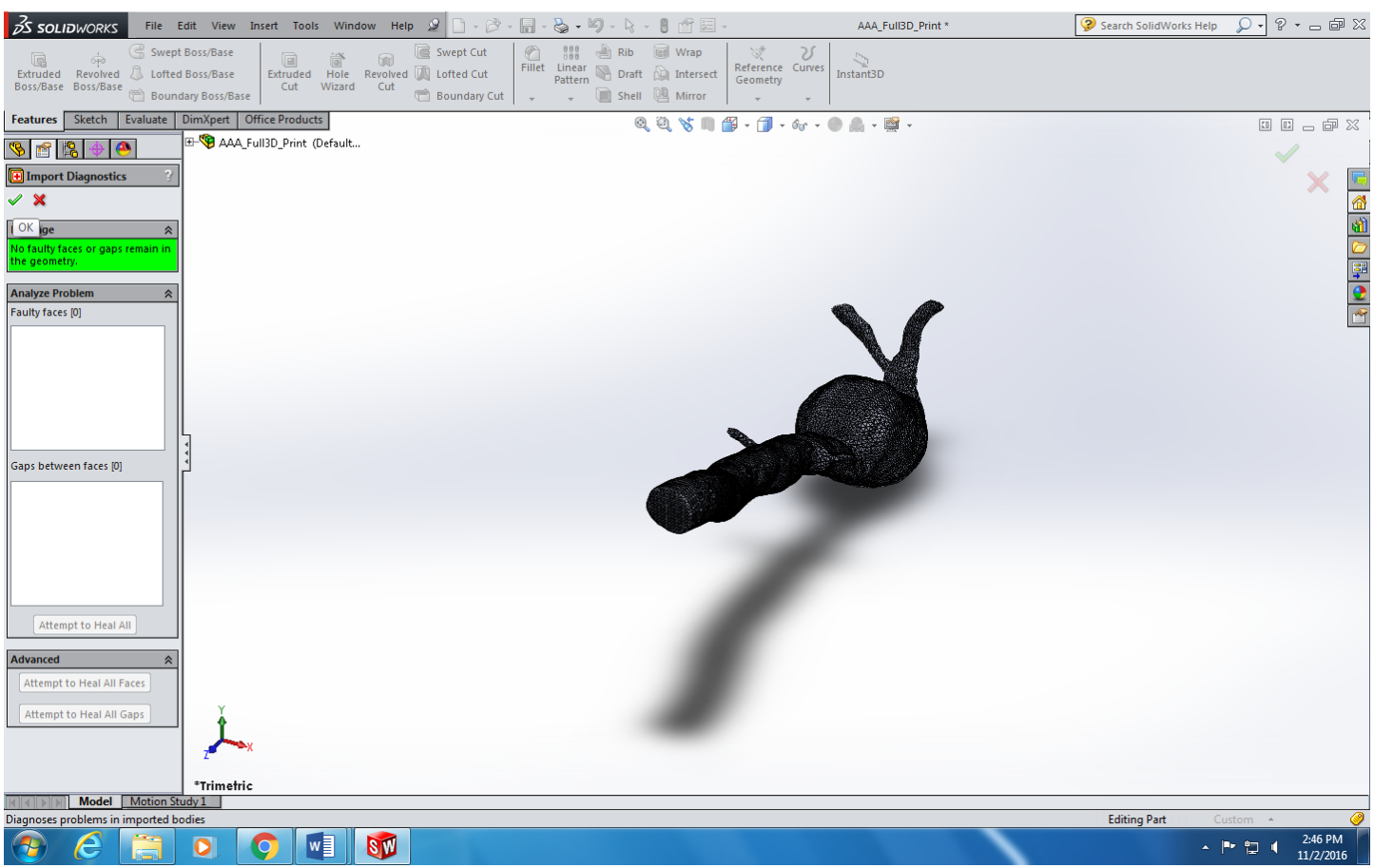


7. Right click the front plane, and choose Normal To.

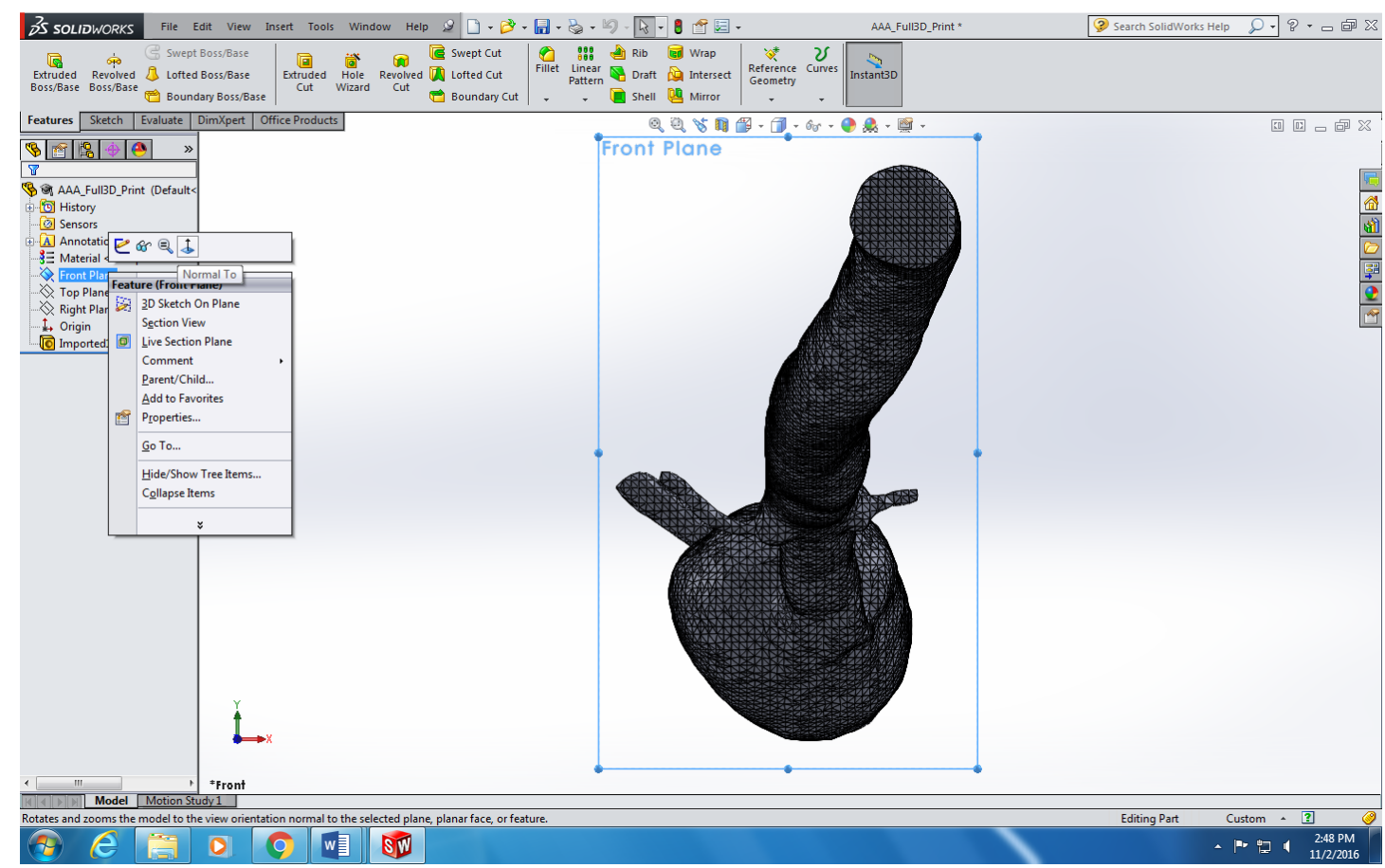


8. Create a new plane parallel to the top of the aneurysm model.

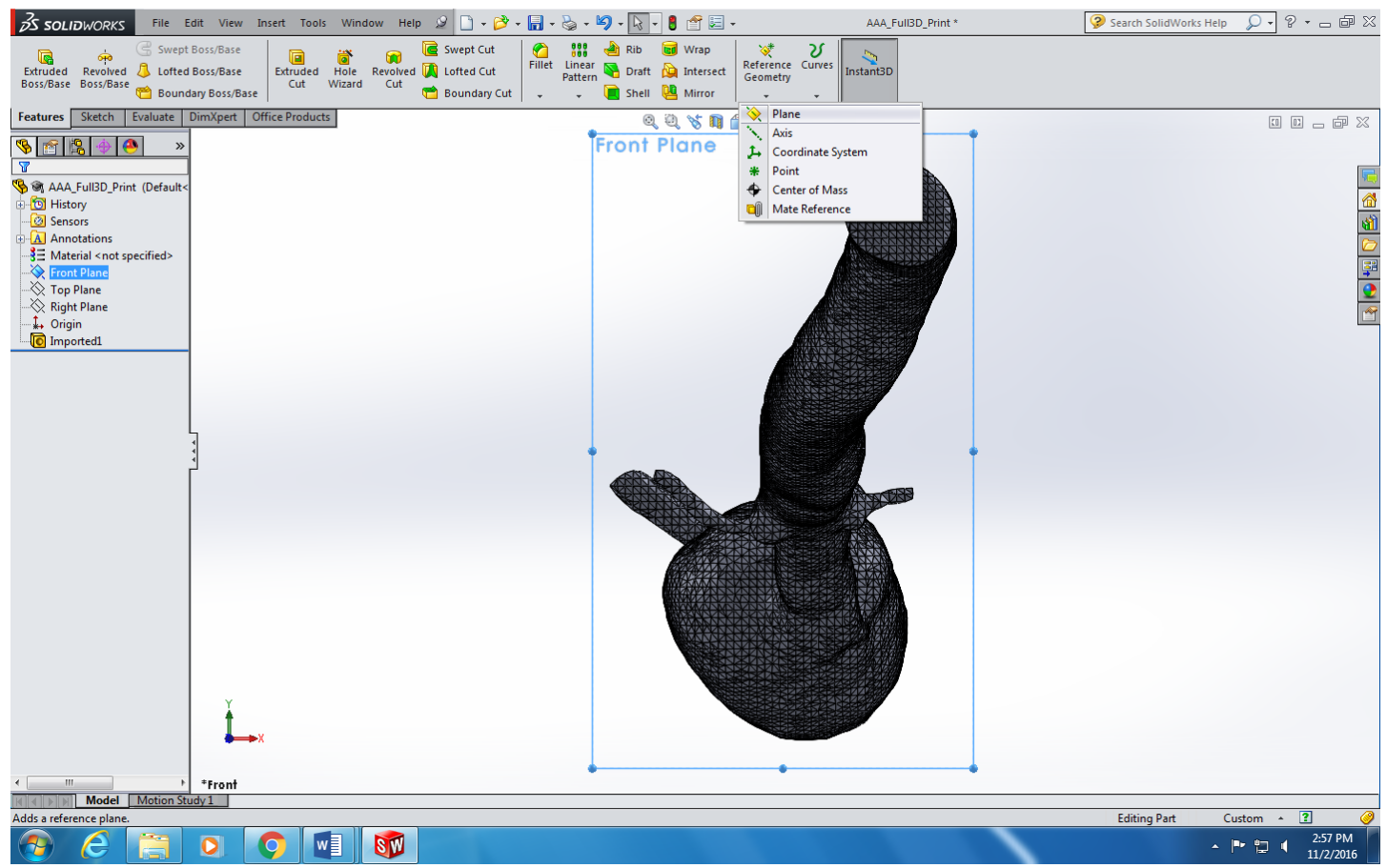


9. Use the Front Plane as the reference plane, and select the new plane to be above to model. Select OK.

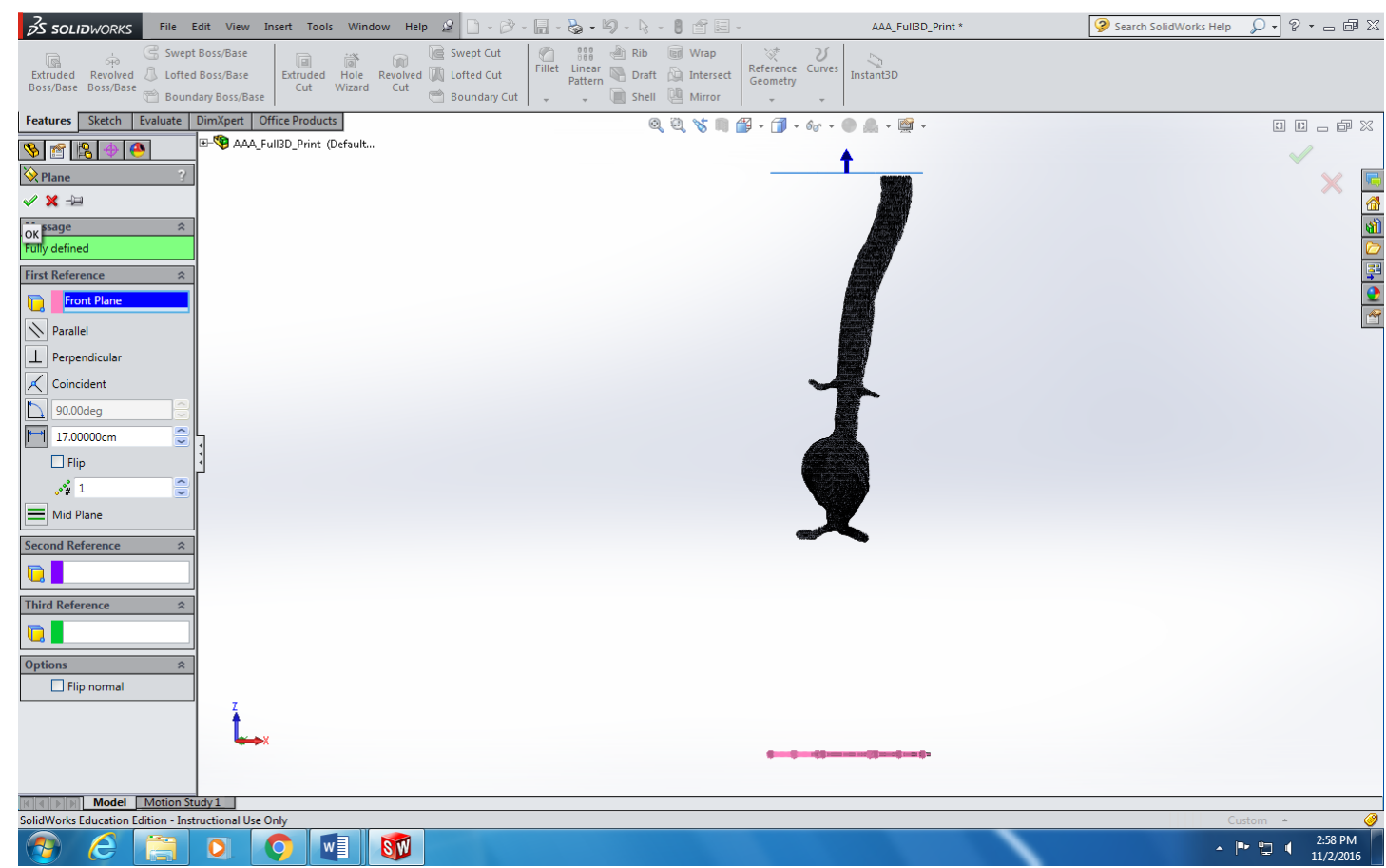


10. Right click on the new plane, and select Normal To

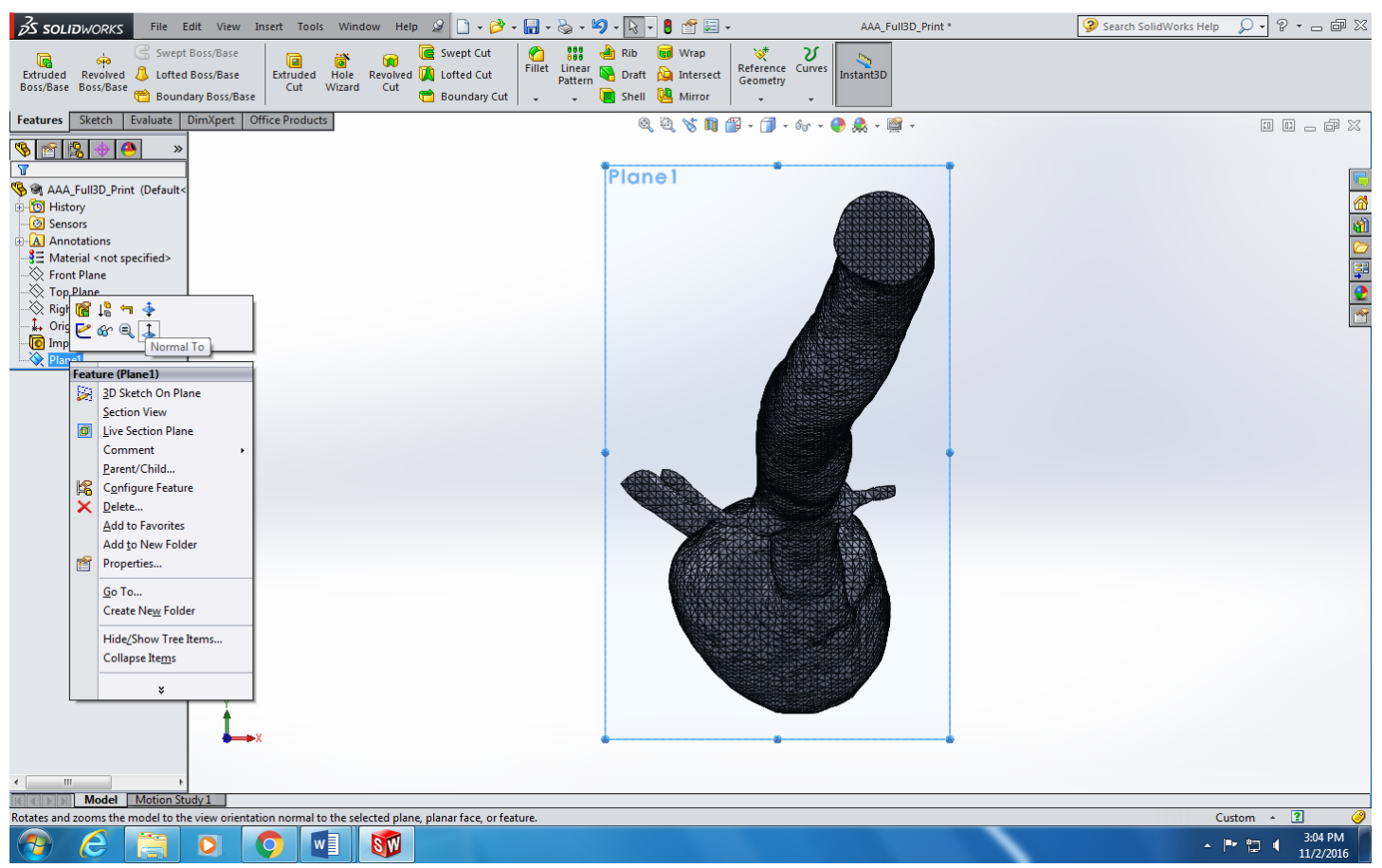


11. Create a Rectangle on the new plane. Sketch $>$ Corner Rectangle
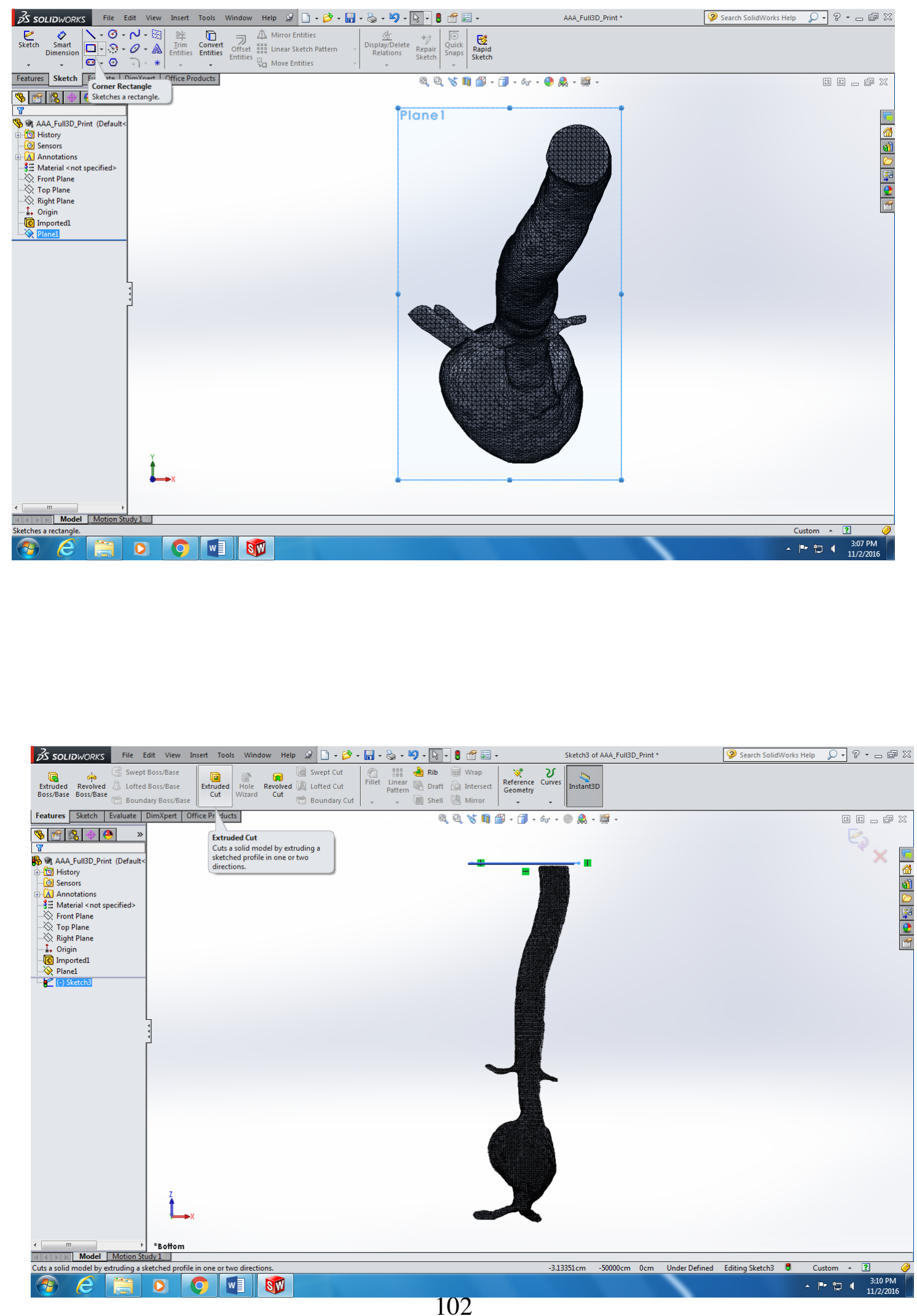
12. Make an extruded cut using the rectangle. Features $>$ Extruded Cut

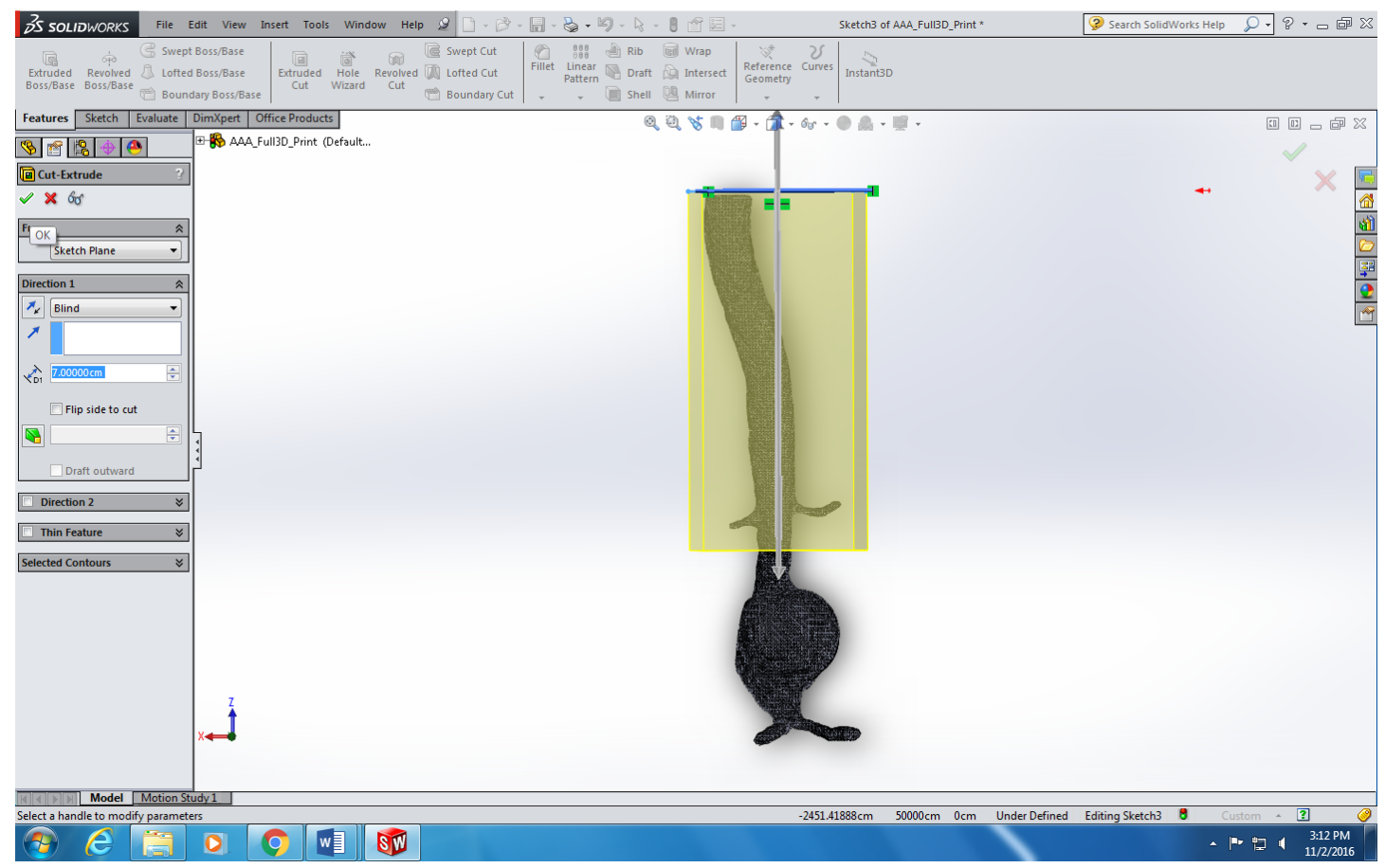

13. Cut the aorta just below the renal arteries. 
14. Repeat this process for both of the legs.

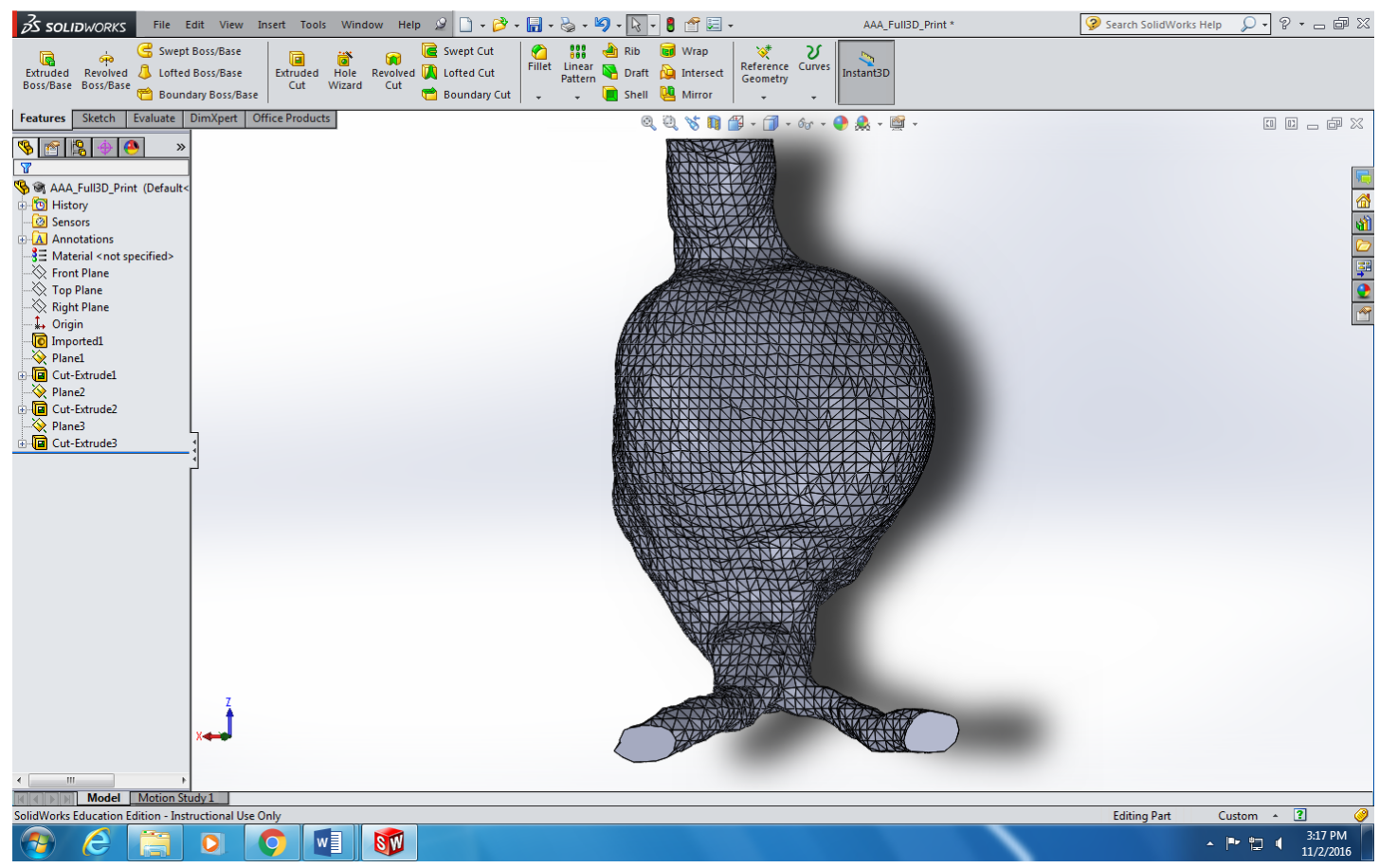


Appendix D: Reducing the Number of Faces on AAA Model

1. Open MeshLab

2. Import the STL file

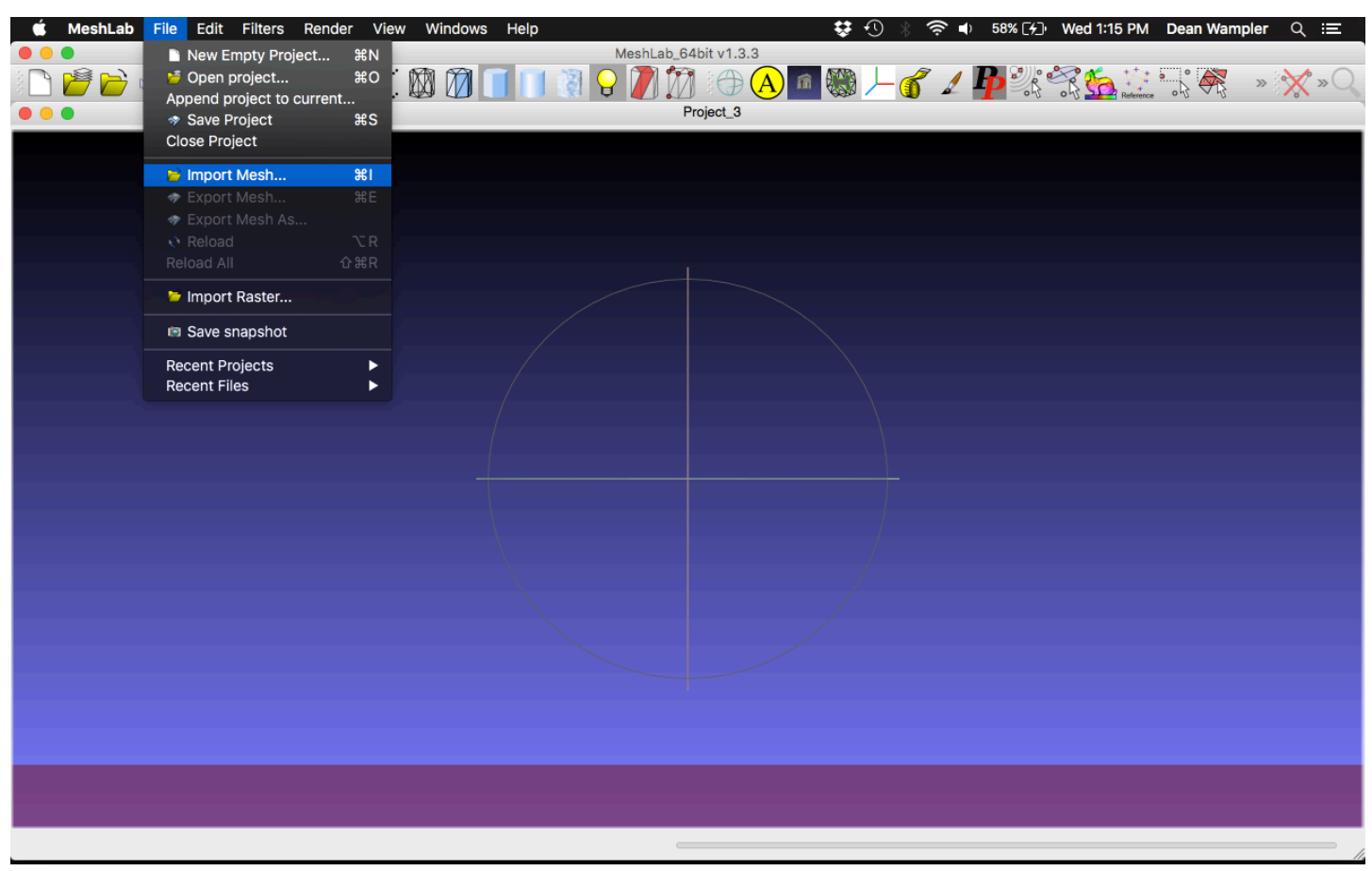


3. Select Unify Duplicated Vertices and click OK

c. MeshLab File Edit Filters Render View Windows Help 35 (1) ร * 64\%[4] Wed 1:23 PM Dean Wampler $Q$ :

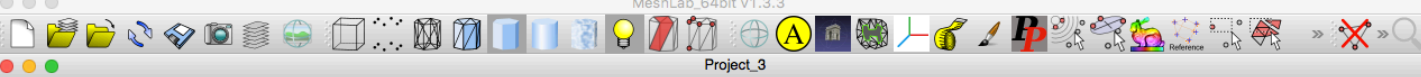

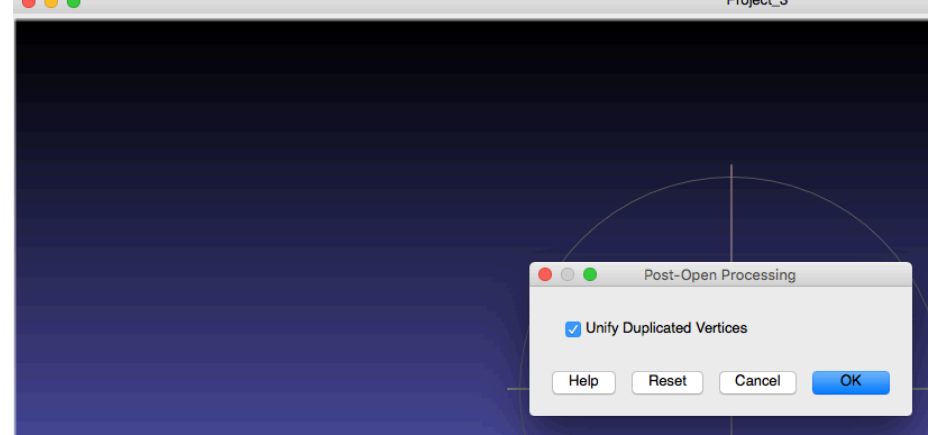


4. Filters $>$ Remeshing, Simplification, and Reconstruction $>$ Quadratic Edge

Collapse Decimation

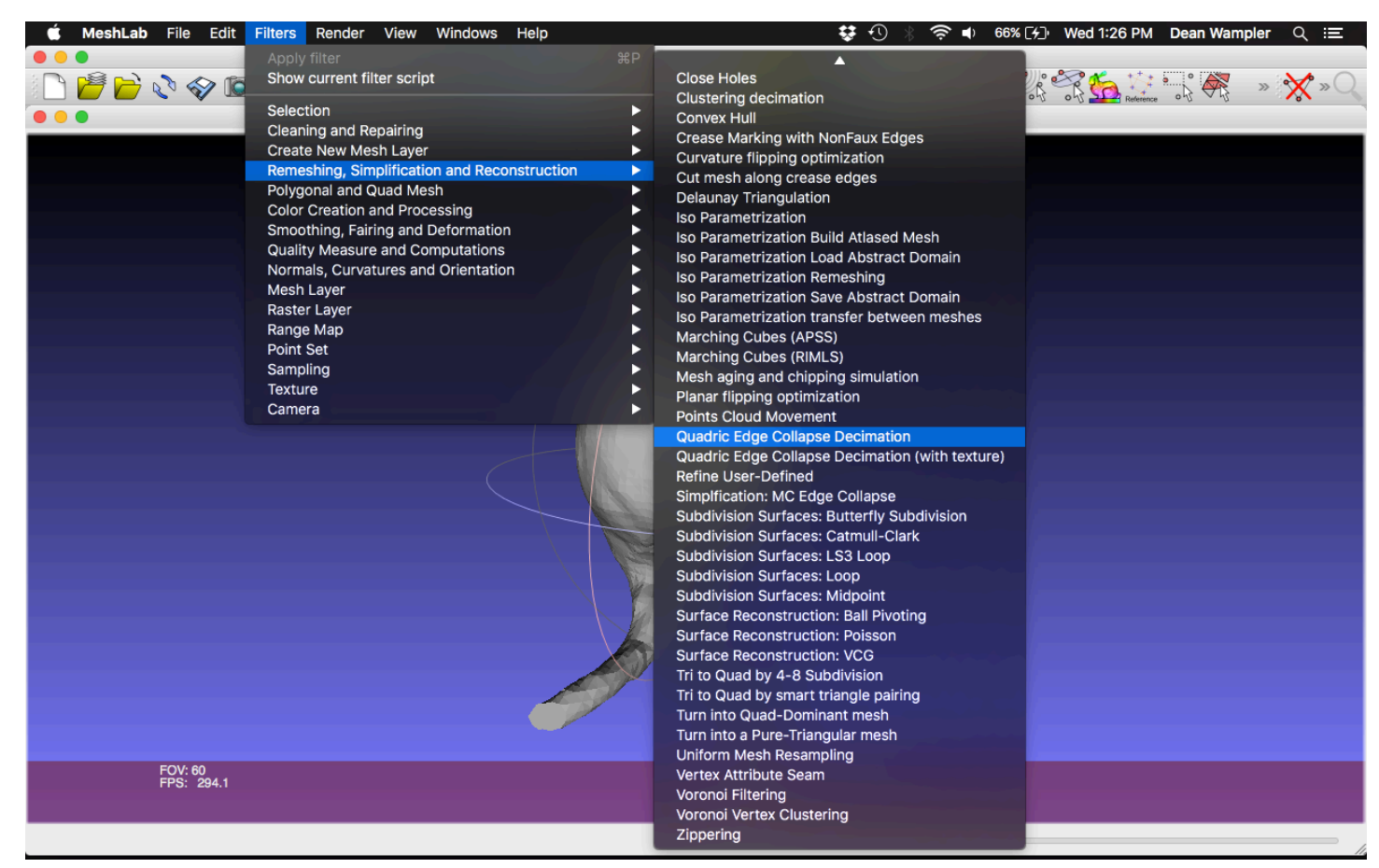


5. Select the percentage reduction and enter 0.50. Check the following: Preserve Normal, Preserve Topology, Optimal position of simplified vertices, and Postsimplification cleaning.

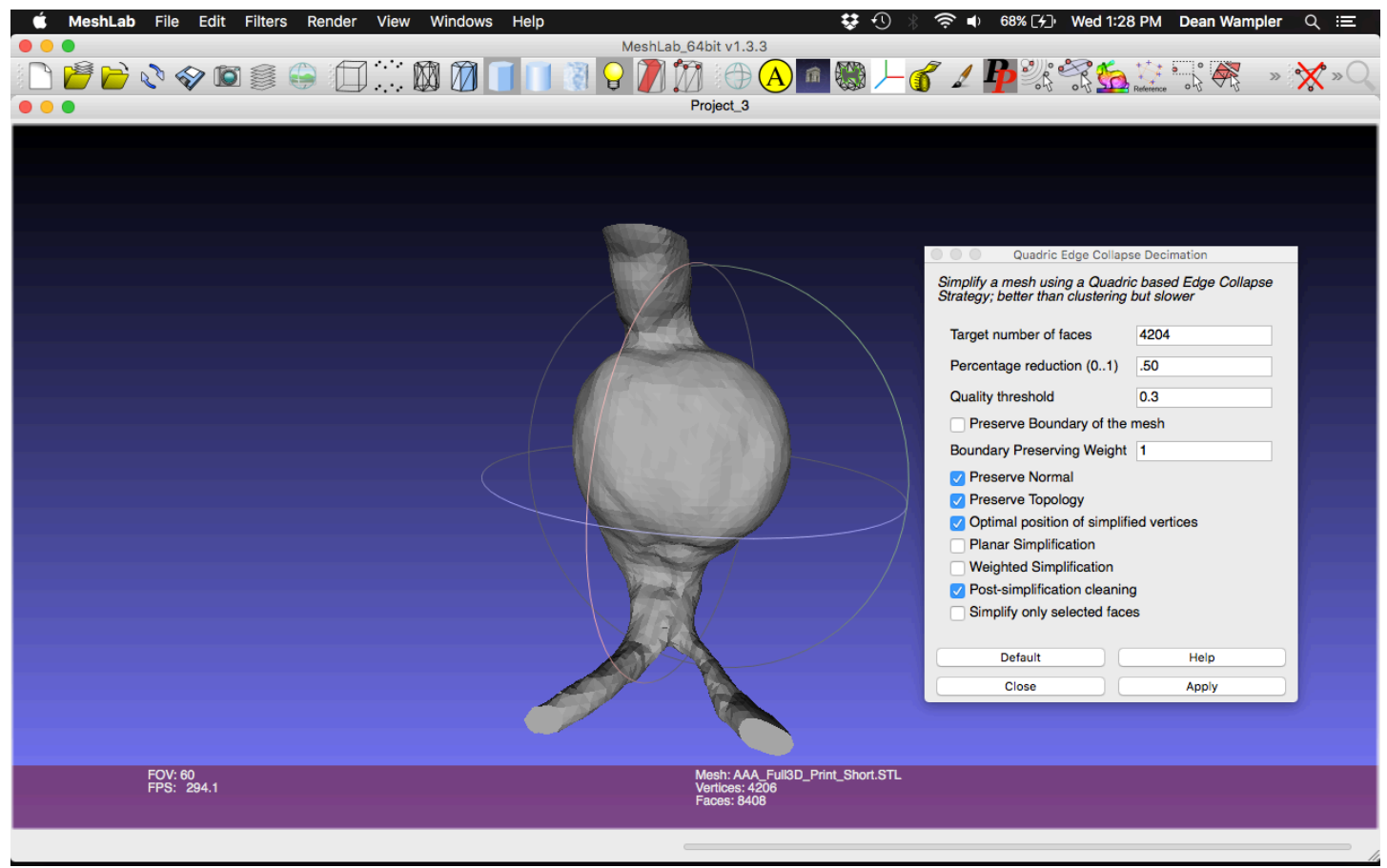

6. Select Apply. Notice that the number of vertices is half of what it was before the operation. 
7. File $>$ Export Mesh As

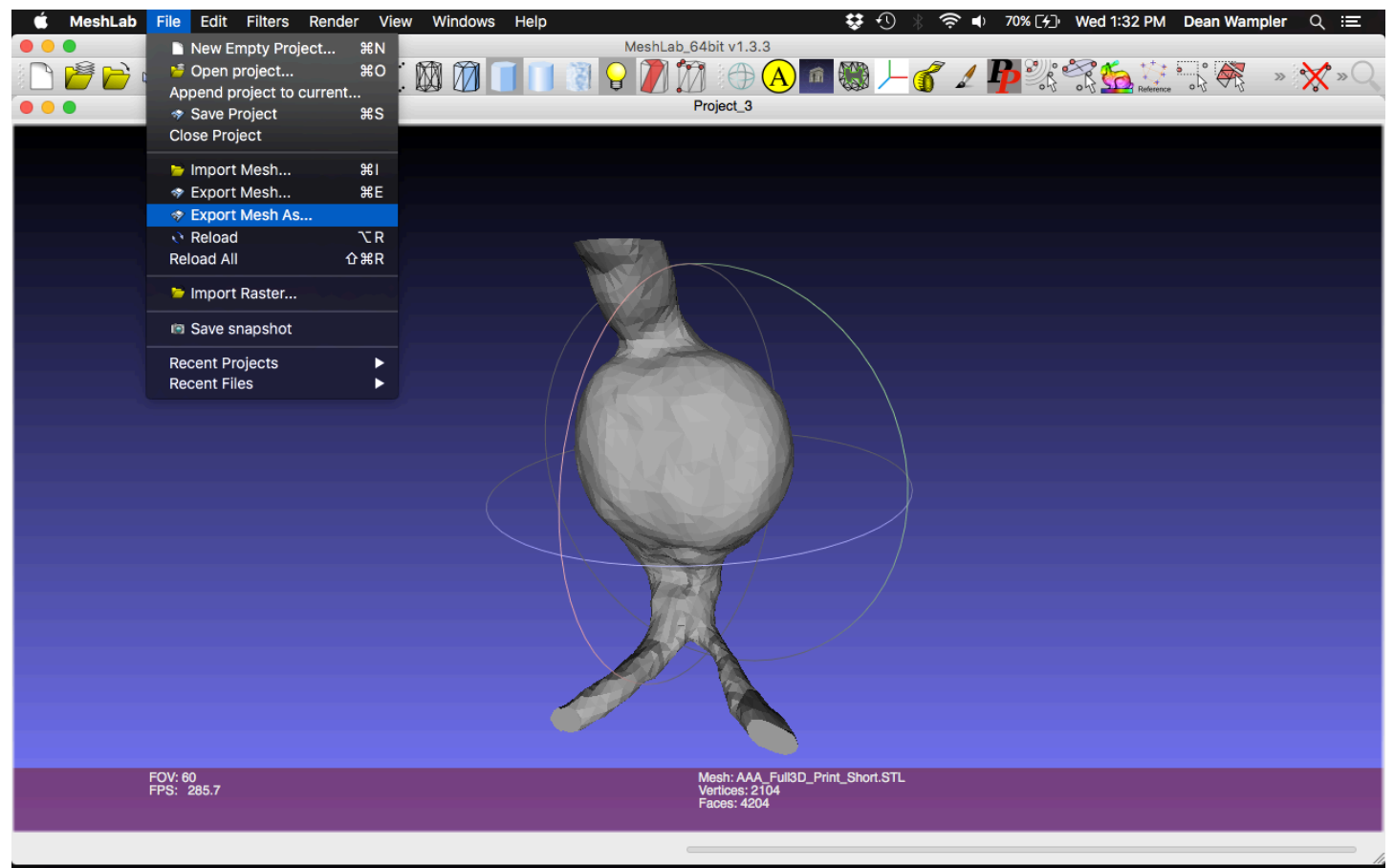

8. Save the mesh as a .STL file

c. MeshLab File Edit Filters Render View Windows Help

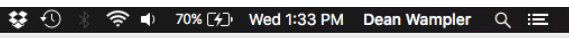

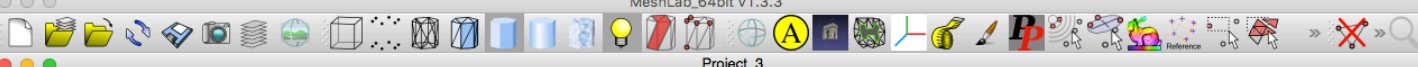

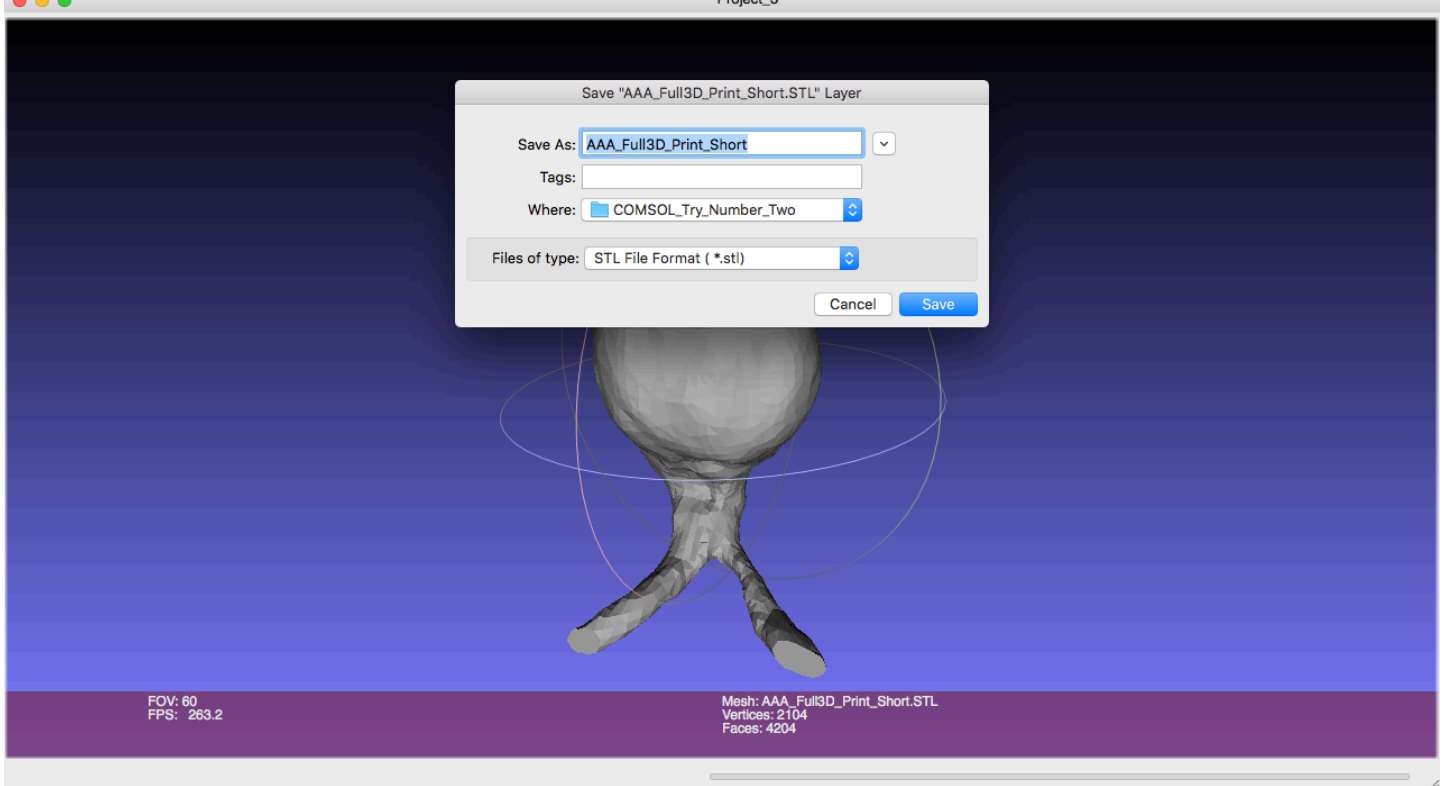




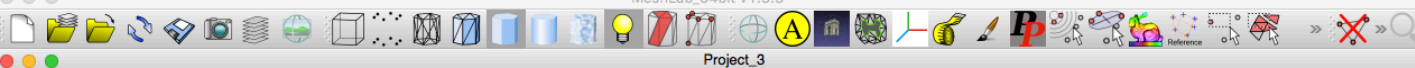

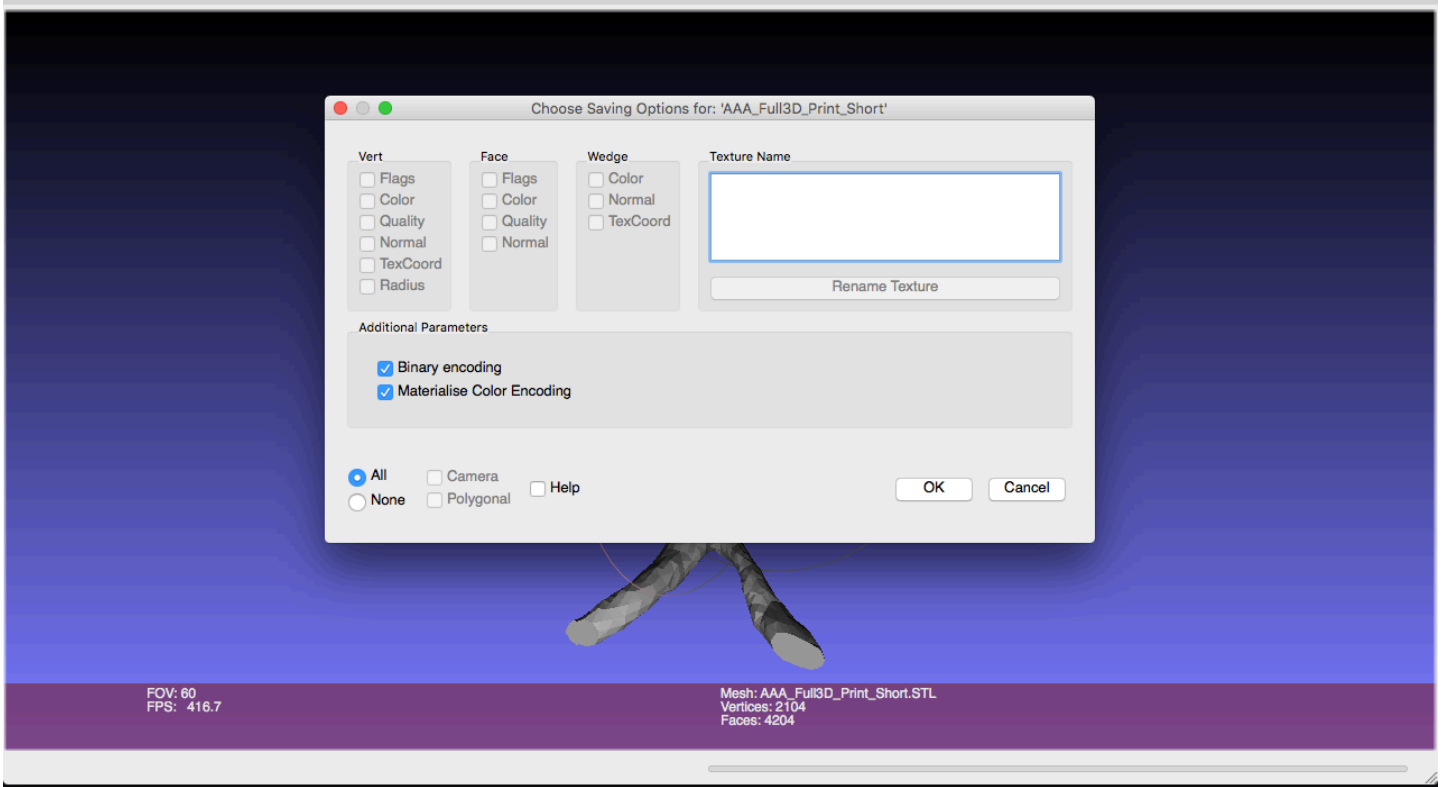




\section{Appendix E: Scaling the AAA Model}

1. Import the STL file into Solidworks as a Solid body

2. Run Import Diagnostics

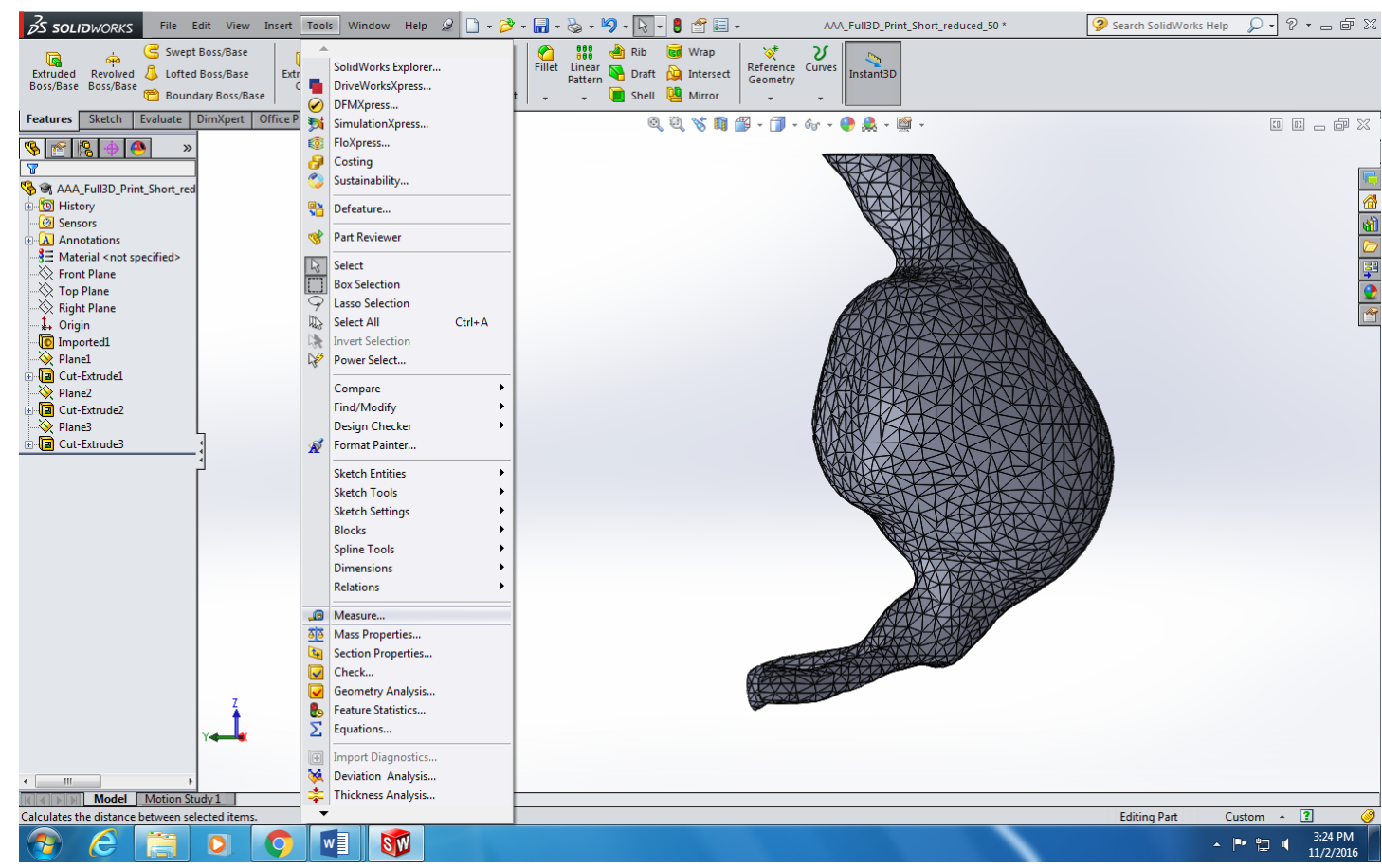


3. Measure the length of the top of the neck of the aneurysm. Tools $>$ Measure

4. Click the left most point of the neck of the aneurysm, and then click the right most point to get the measurement across the neck of the model.

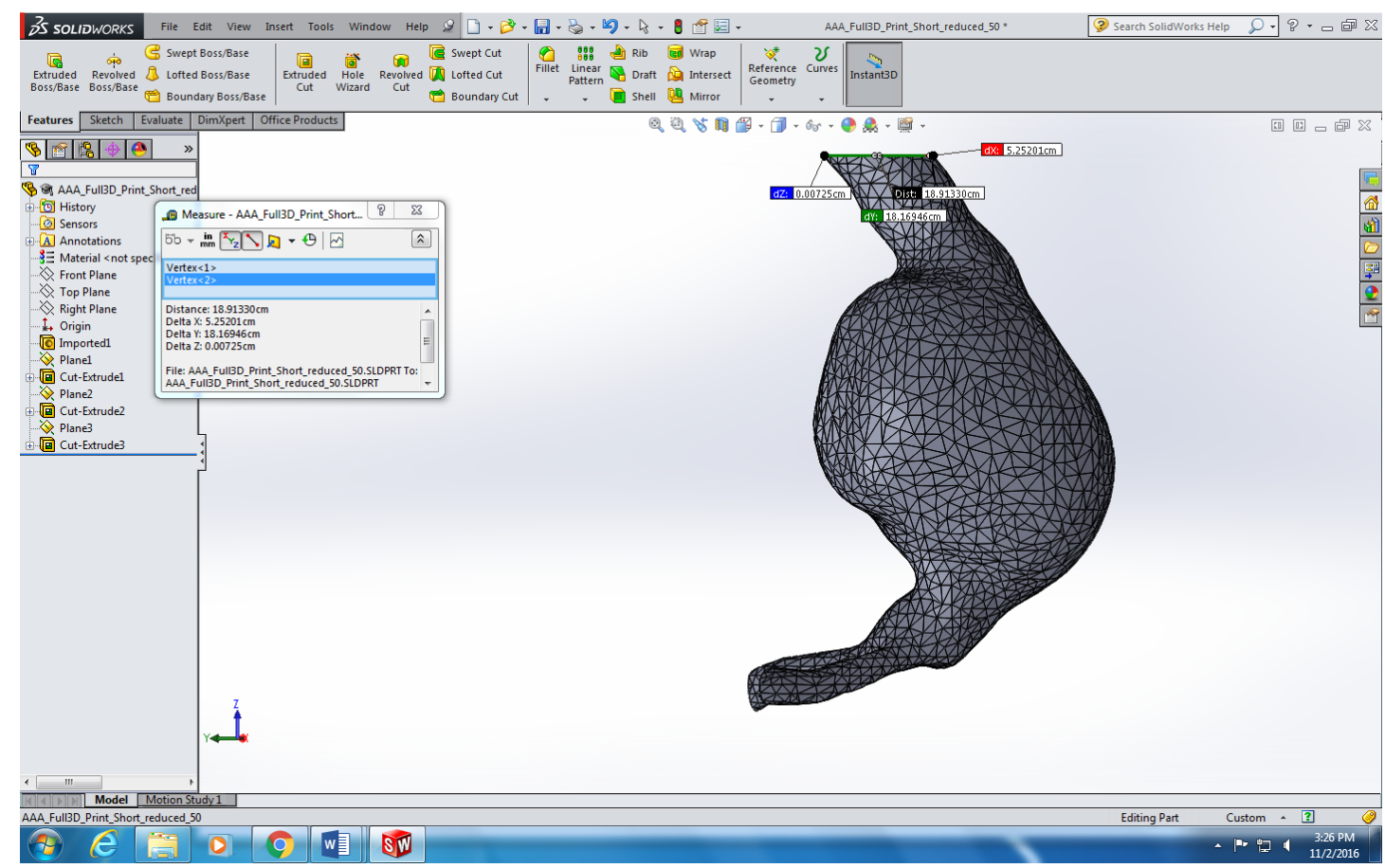


5. The model is $10 \mathrm{X}$ larger than it should be. Scale the model by a factor of $1 / 10$ to get the actual size of the model. Insert $>$ Features $>$ Scale

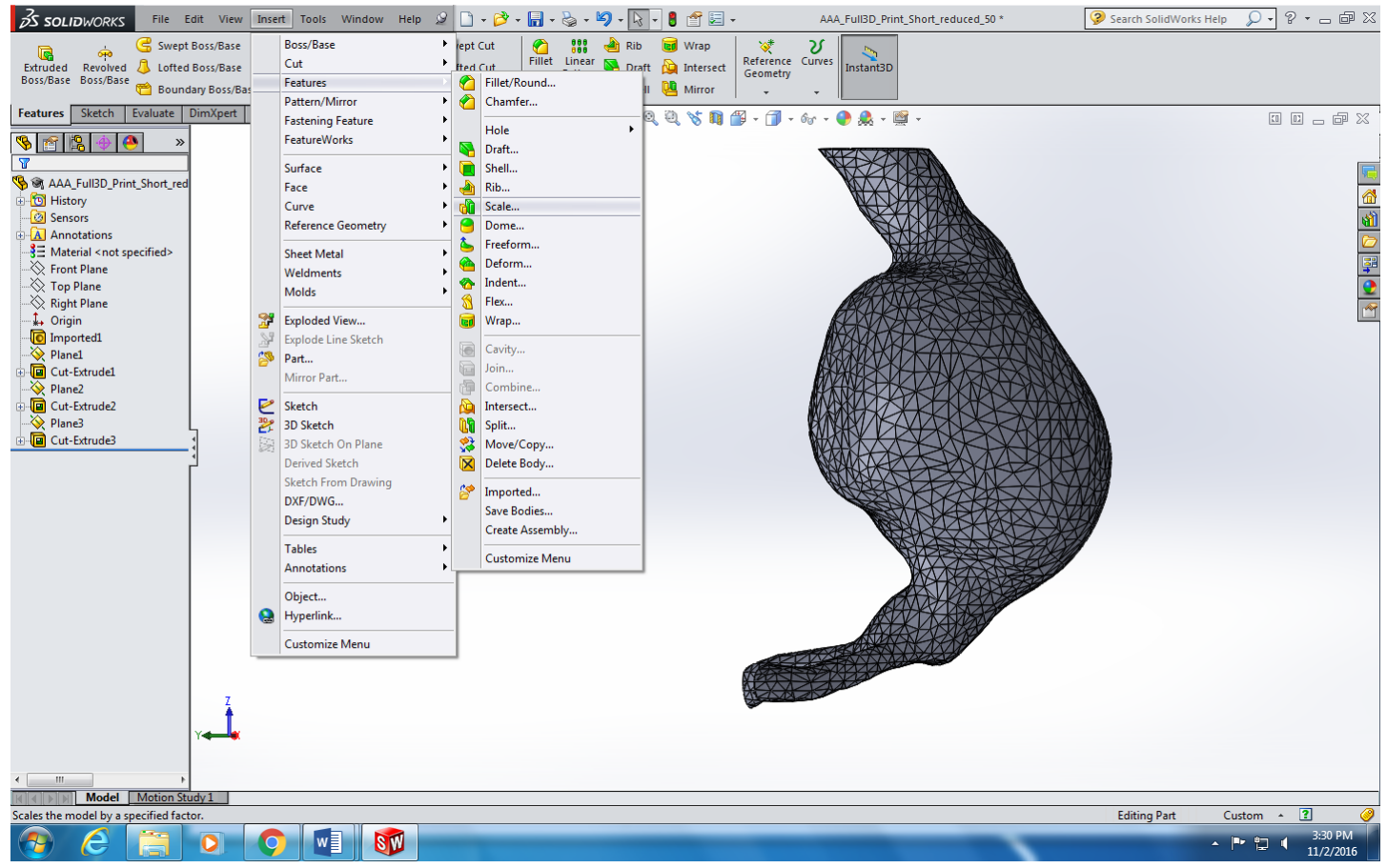


6. Scale about the Centroid, select uniform scaling, and enter 0.1 as the scale value.

Select OK.

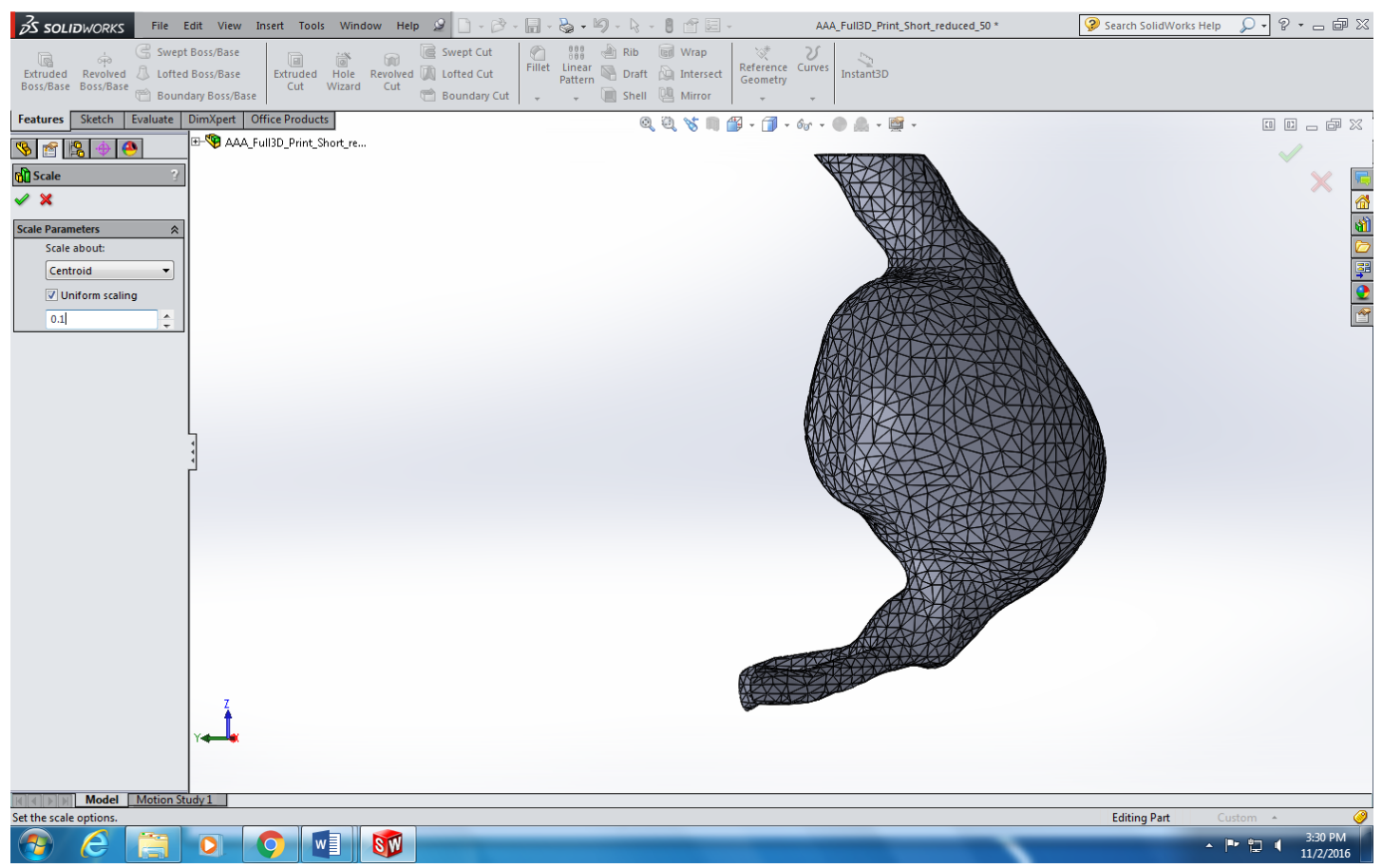


7. The model is now the correct size.

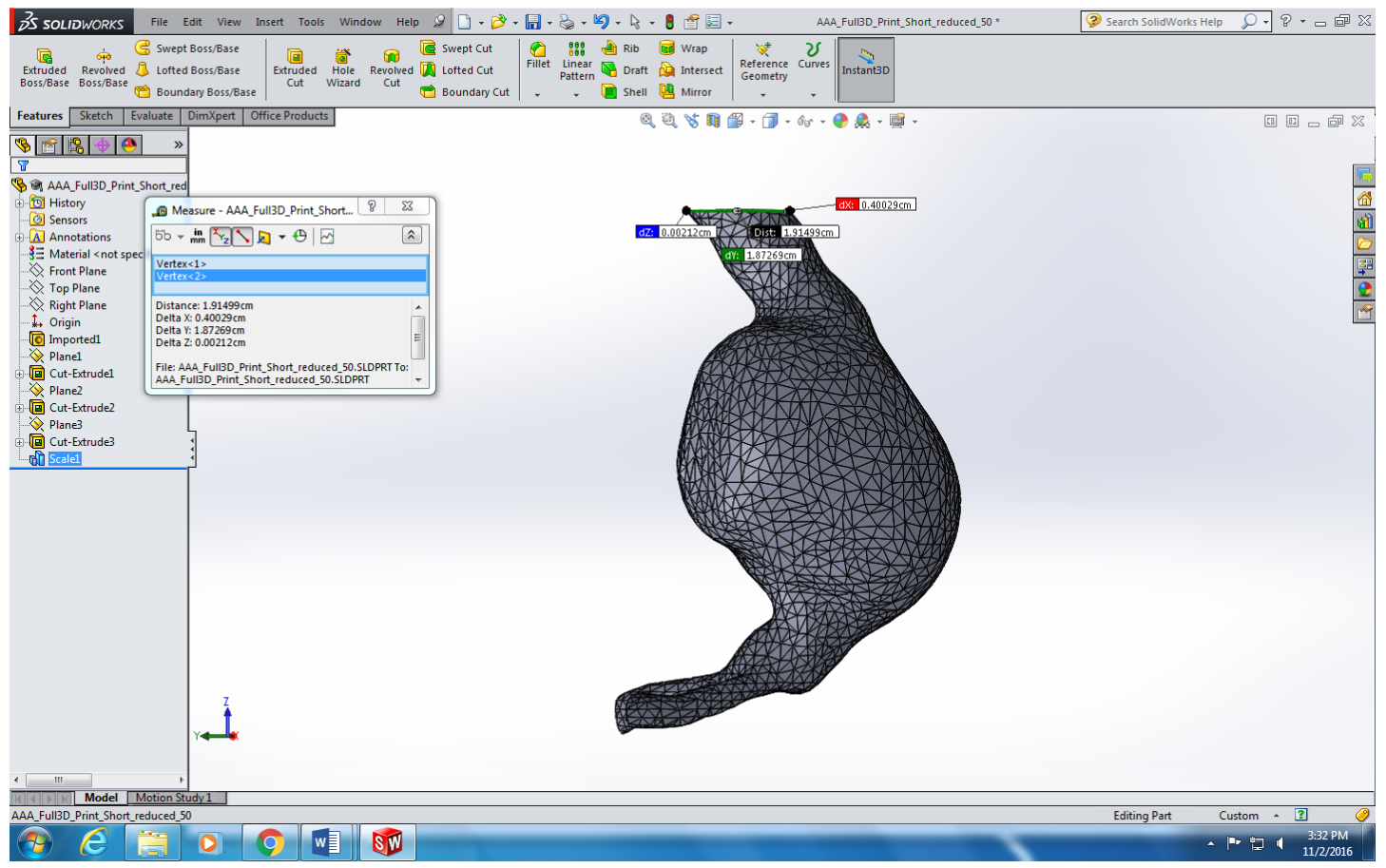


8. Save the file as a IGES file to be imported into COMSOL Multiphysics ${ }^{\circledR}$

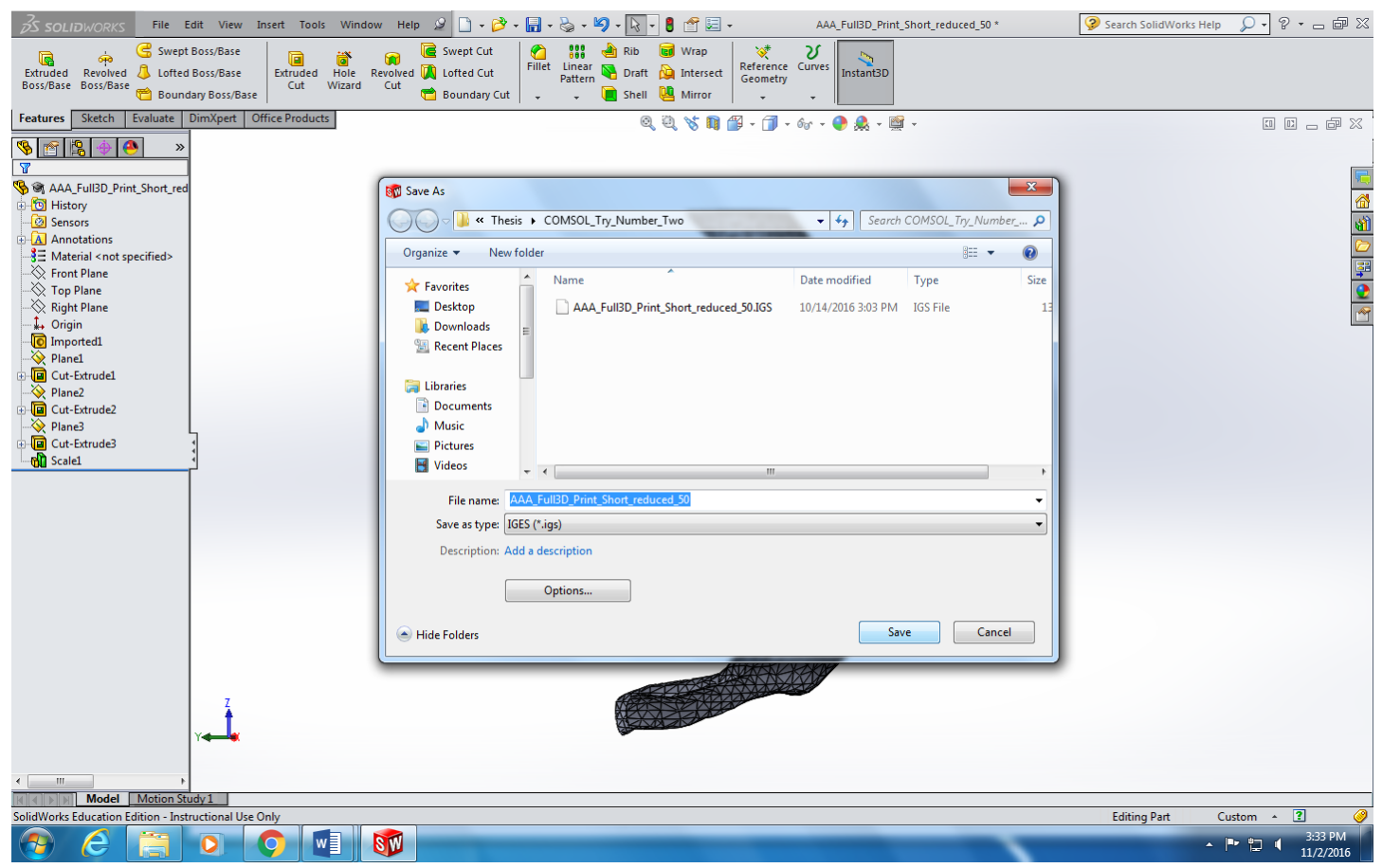




\section{Appendix F: Velocity Calculations}

$$
\begin{gathered}
\frac{1 L}{15.094 \mathrm{~s}}\left(\frac{60 \mathrm{~s}}{1 \mathrm{~min}}\right)=3.97508 \frac{\mathrm{L}}{\mathrm{min}} \\
Q=3.97508 \frac{\mathrm{L}}{\min }\left(\frac{1 \mathrm{~min}}{60 \mathrm{~s}}\right)\left(\frac{1 \mathrm{~m}^{3}}{1000 \mathrm{~L}}\right) \\
Q=6.62514 \times 10^{-5} \frac{\mathrm{m}^{3}}{\mathrm{~s}} \\
Q=v A=v \pi r^{2} \\
v_{\max }=2 * v_{a v g}=2 * 26.035 \frac{\mathrm{cm}}{\mathrm{s}}=52.07 \frac{\mathrm{cm}}{\mathrm{s}} \\
6.62514 \times 10^{-5} \frac{\mathrm{m}^{3}}{\mathrm{~s}}=v\left[\pi(0.009 \mathrm{~m})^{2}\right] \\
=0.26035 \frac{\mathrm{m}}{\mathrm{s}}=26.035 \frac{\mathrm{cm}}{\mathrm{s}}
\end{gathered}
$$




\section{Appendix G: Flow Visualization Conversion}

1. Import Images to ImageJ

2. Convert individual images to a stack. Image $>$ Stacks $>$ Images to Stack

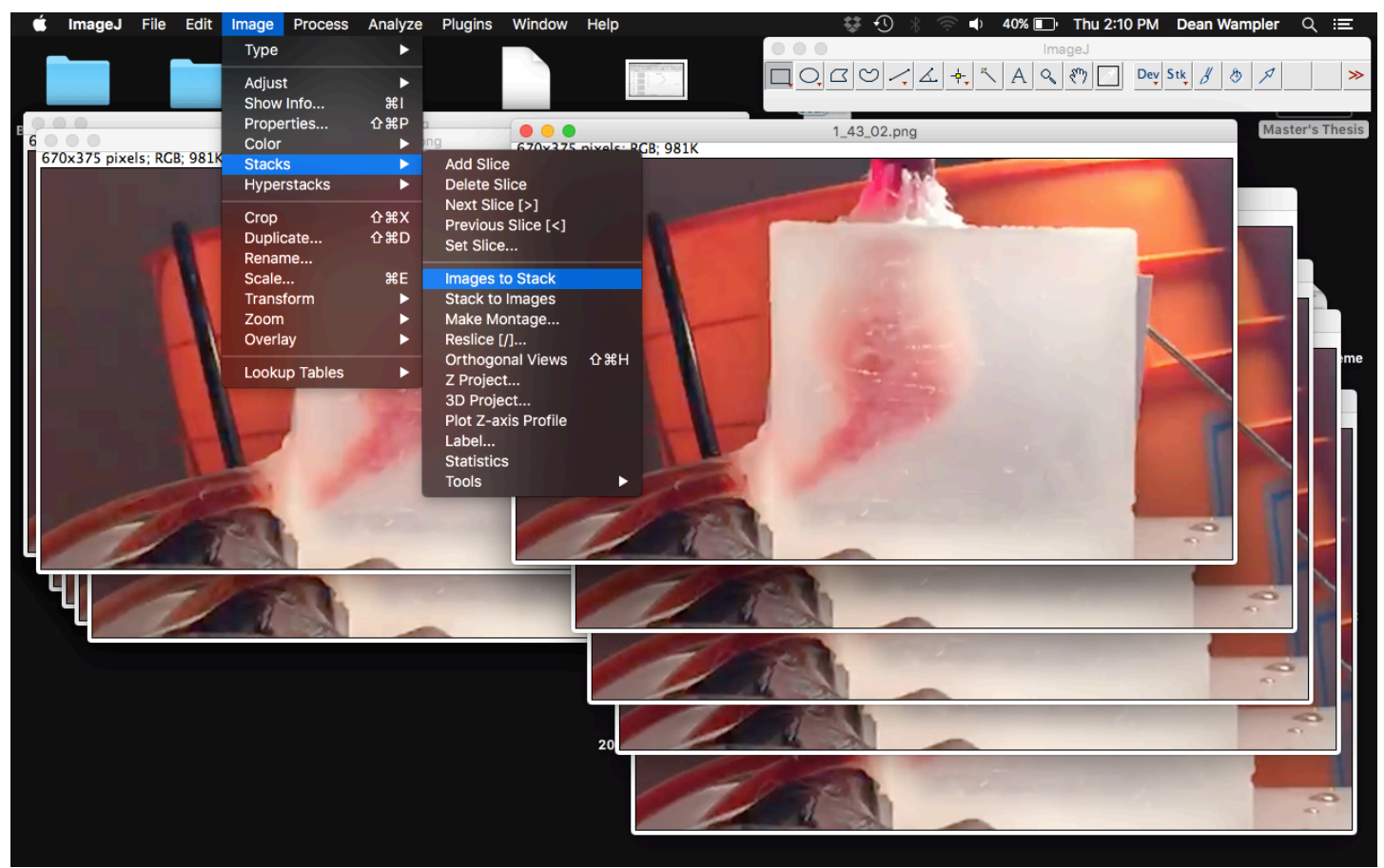


3. Set the scale of the stack to convert from pixels to centimeters. Use the line tool to measure from the left side of the PDMS mold to the right side of the PDMS mold. Analyze $>$ Set Scale

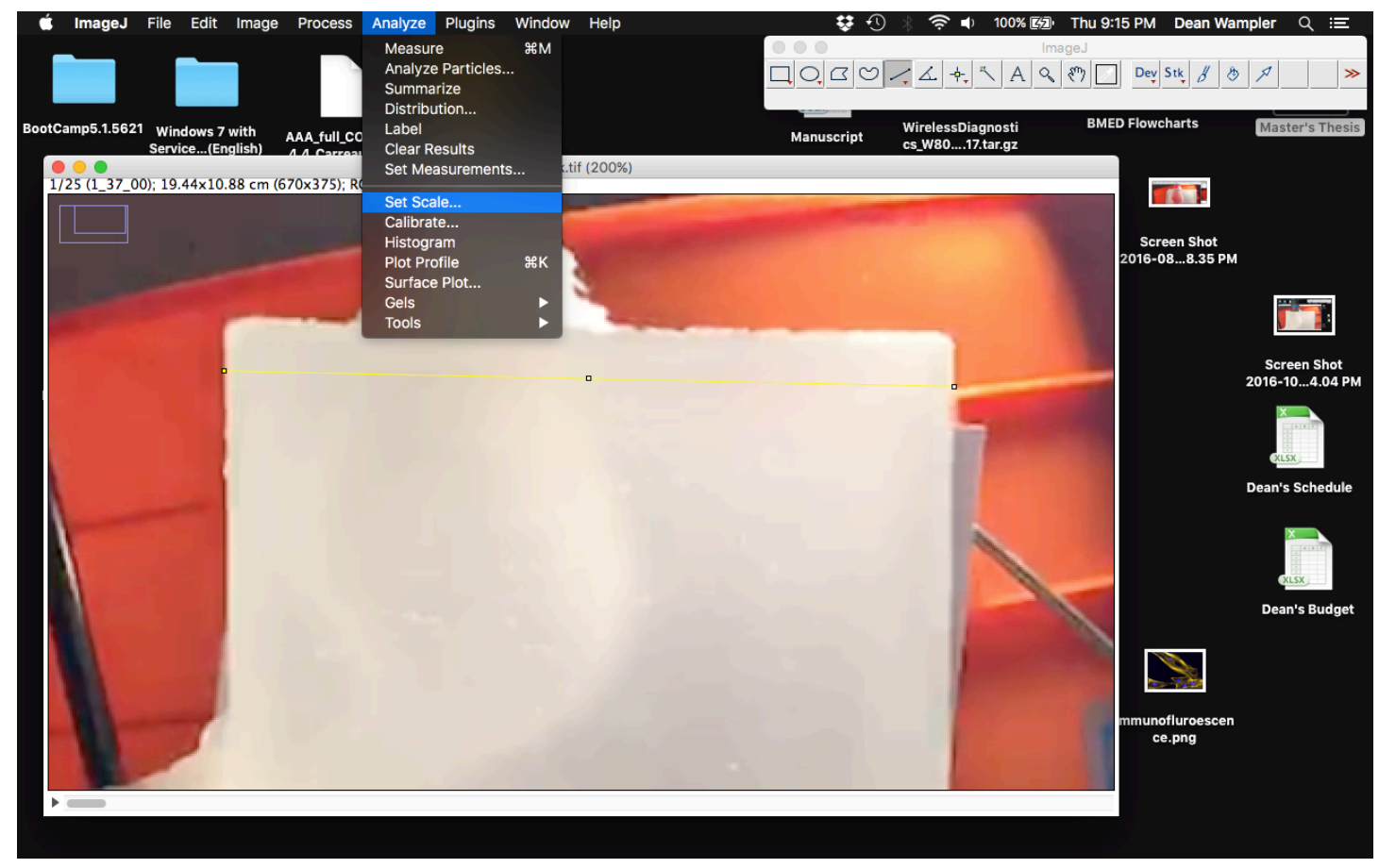


4. The known distance from the left side to the right side is the $10 \mathrm{~cm}$

\begin{tabular}{|c|c|c|c|}
\hline 000 & \multicolumn{3}{|c|}{ Set Scale } \\
\hline \multicolumn{2}{|c|}{ Distance in pixels: } & 344.5 & \\
\hline \multicolumn{2}{|c|}{ Known distance: } & 10.0 & \\
\hline \multicolumn{2}{|c|}{ Pixel aspect ratio: } & 1.0 & \\
\hline \multicolumn{2}{|c|}{ Unit of length: } & $\mathrm{cm}$ & \\
\hline \multicolumn{4}{|c|}{ Click to Remove Scale } \\
\hline \multicolumn{4}{|c|}{$\square$ Global } \\
\hline \multicolumn{4}{|c|}{ Scale: 34.458 pixels $/ \mathrm{cm}$} \\
\hline Help & & ancel & OK \\
\hline
\end{tabular}

5. Edit the Brightness/Contrast of the stacked images. Images $>$ Adjust $>$

Brightness/Contrast

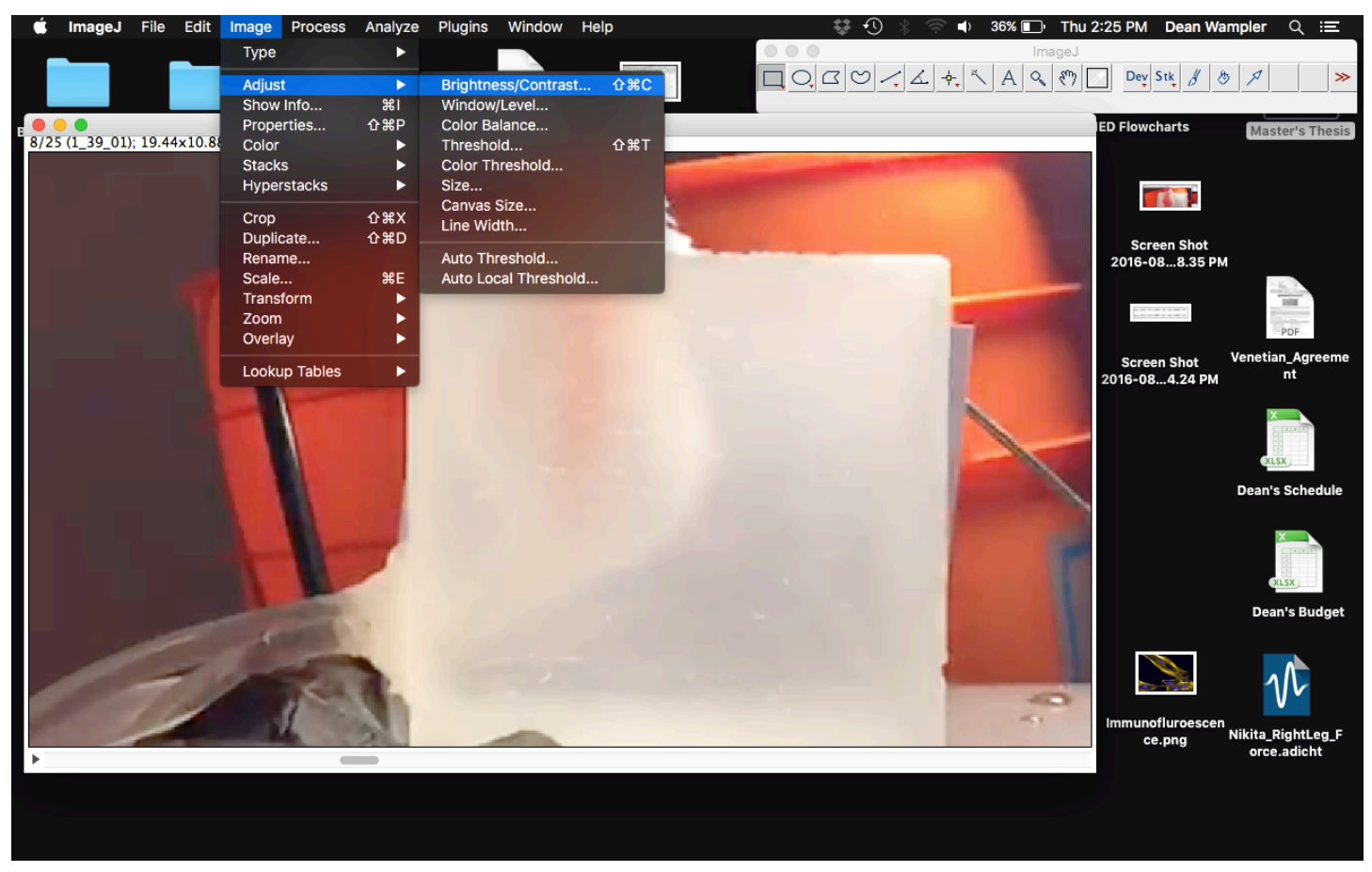


6. Adjust the Minimum and Maximum values to increase the contrast

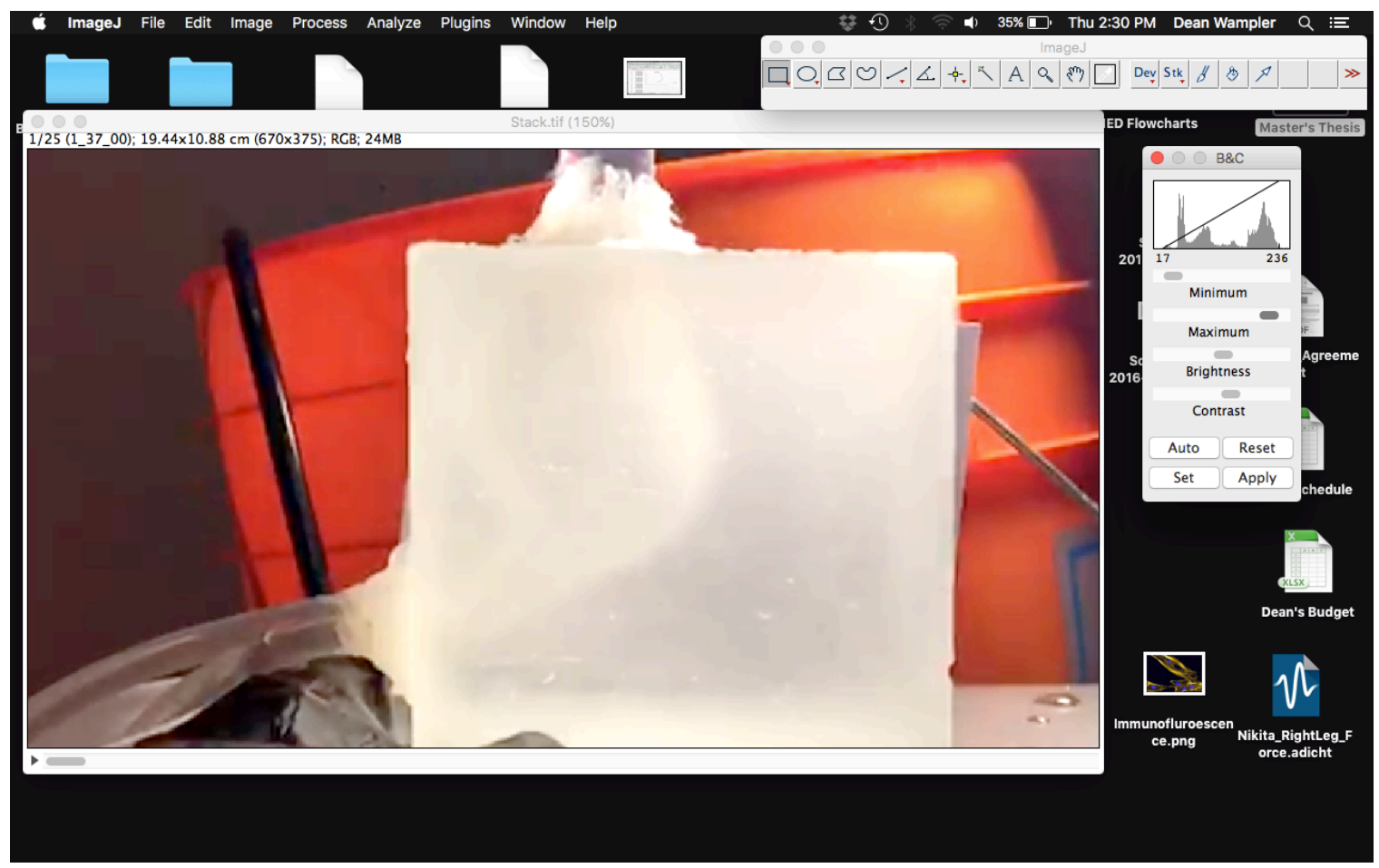


7. Adjust the threshold to allow for better visualization of the dye. Image $>$ Adjust $>$ Threshold

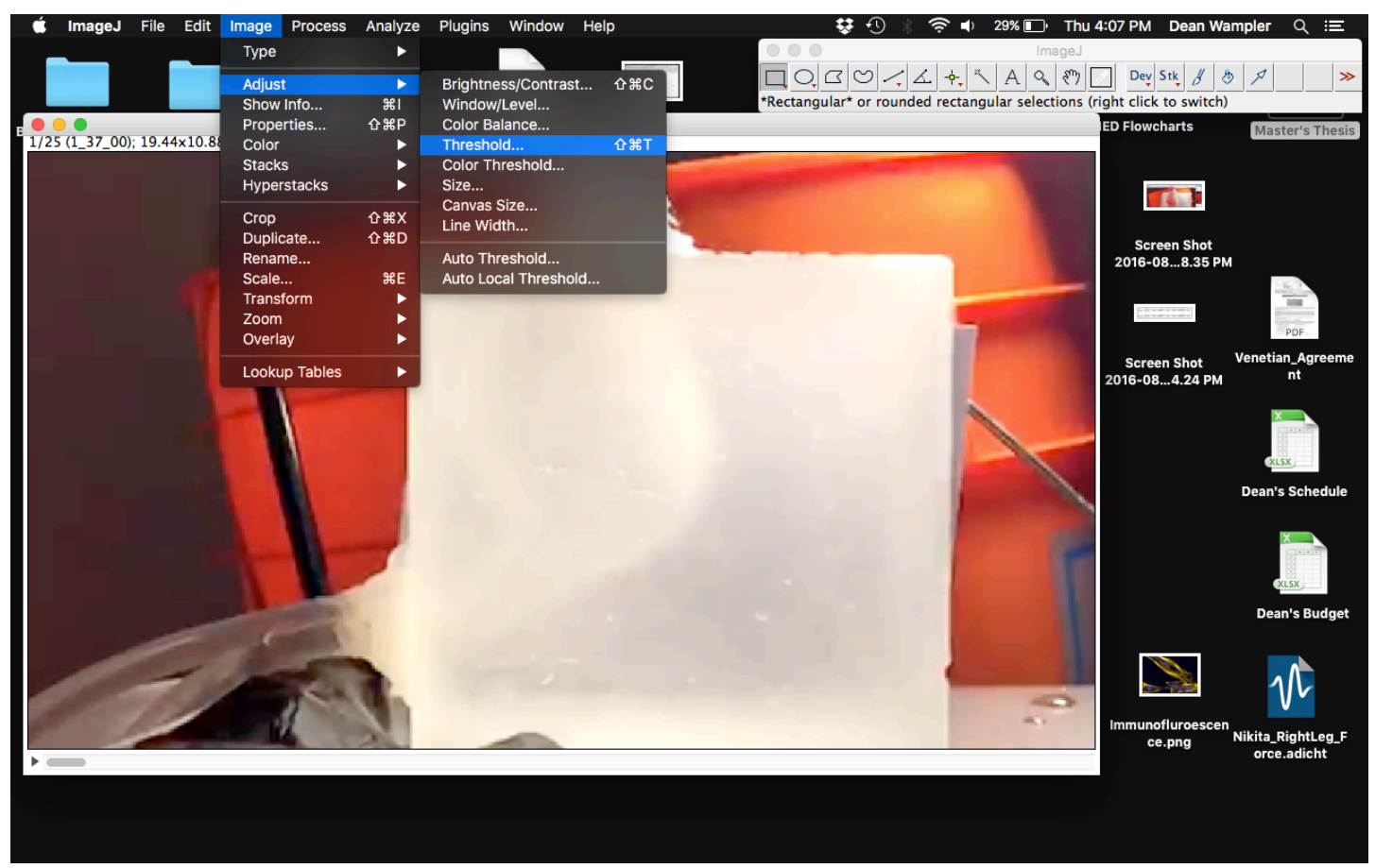


8. Sample the threshold to obtain a relatively appropriate value.

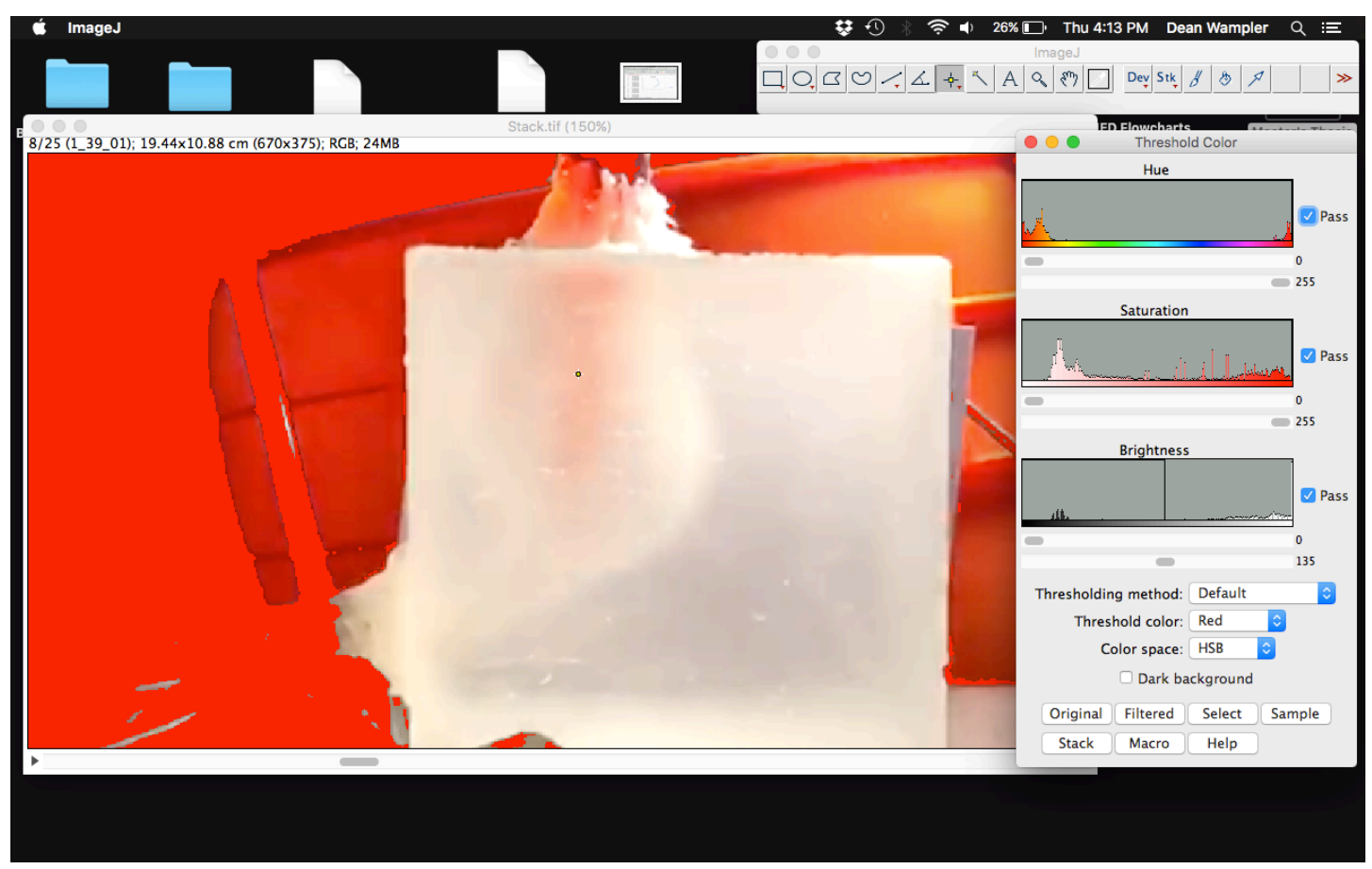


9. Adjust the values for hue, saturation, and brightness to most accurately match the dye front. Select "Stack" to apply the threshold to the entire stack of images.

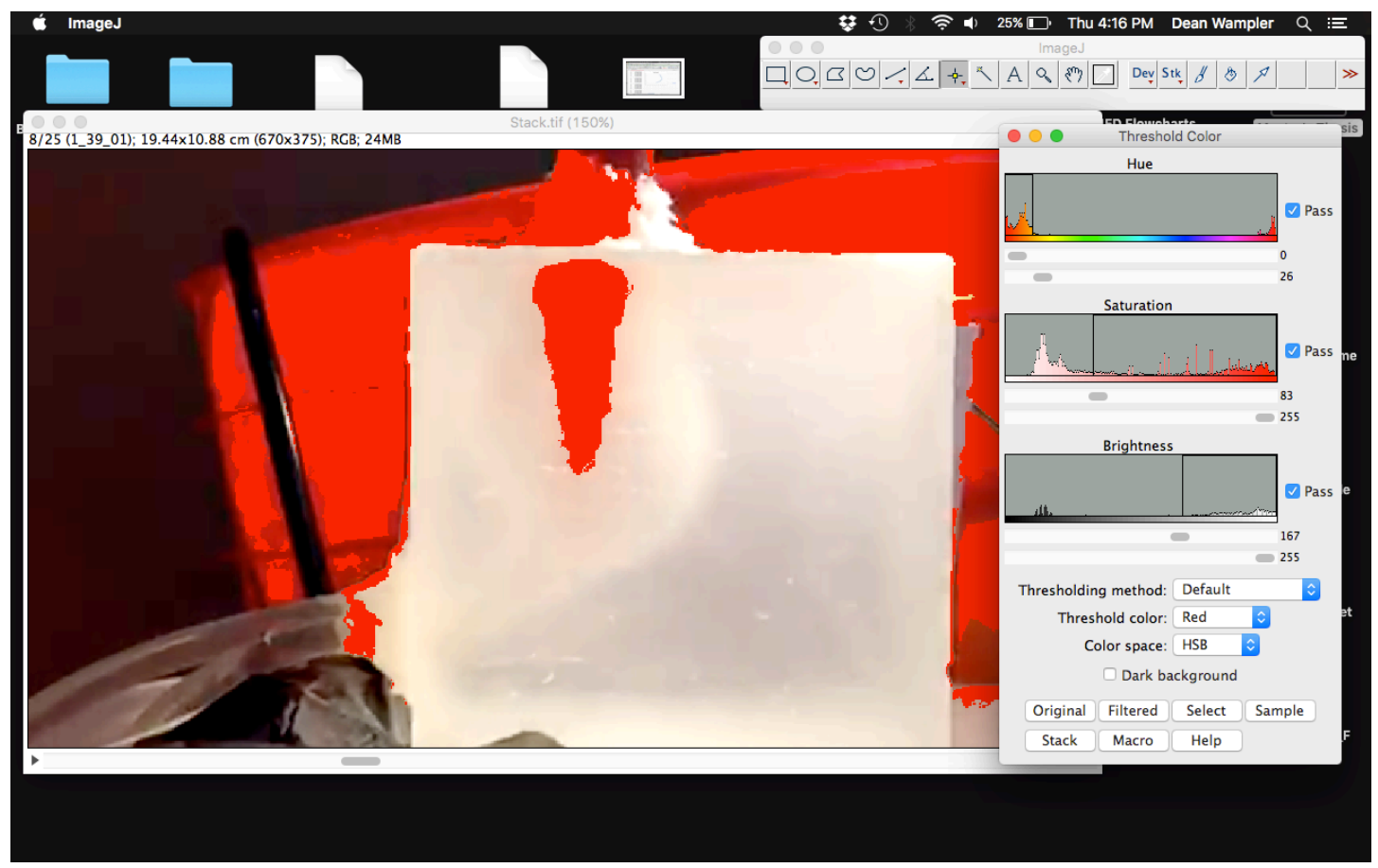


10. Convert the stack back into individual images. Images $>$ Stacks $>$ Stack to Images

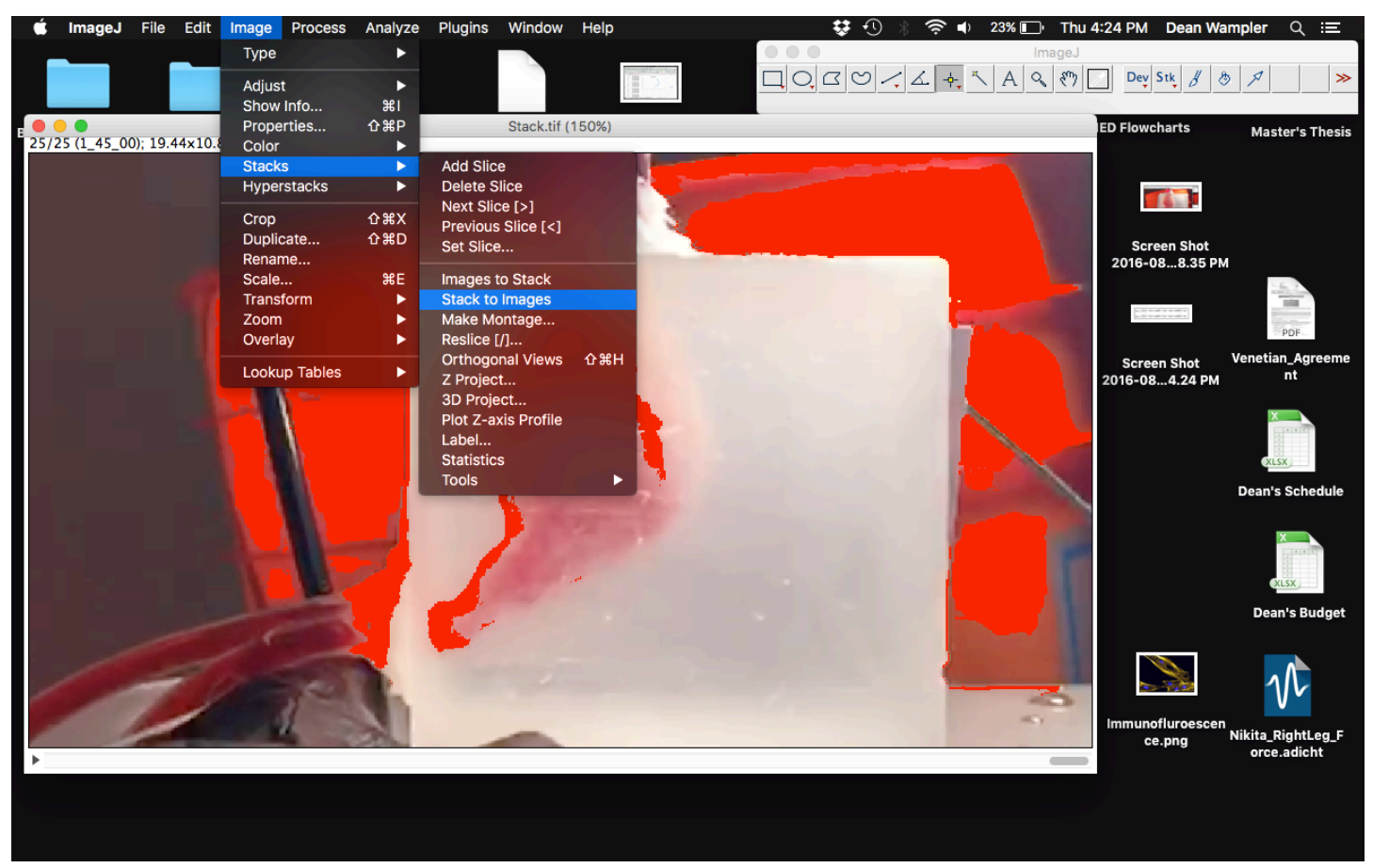


Appendix H: Angle of Rotation

1. Open Screenshots of the COMSOL Multiphysics ${ }^{\circledR}$ geometry in ImageJ

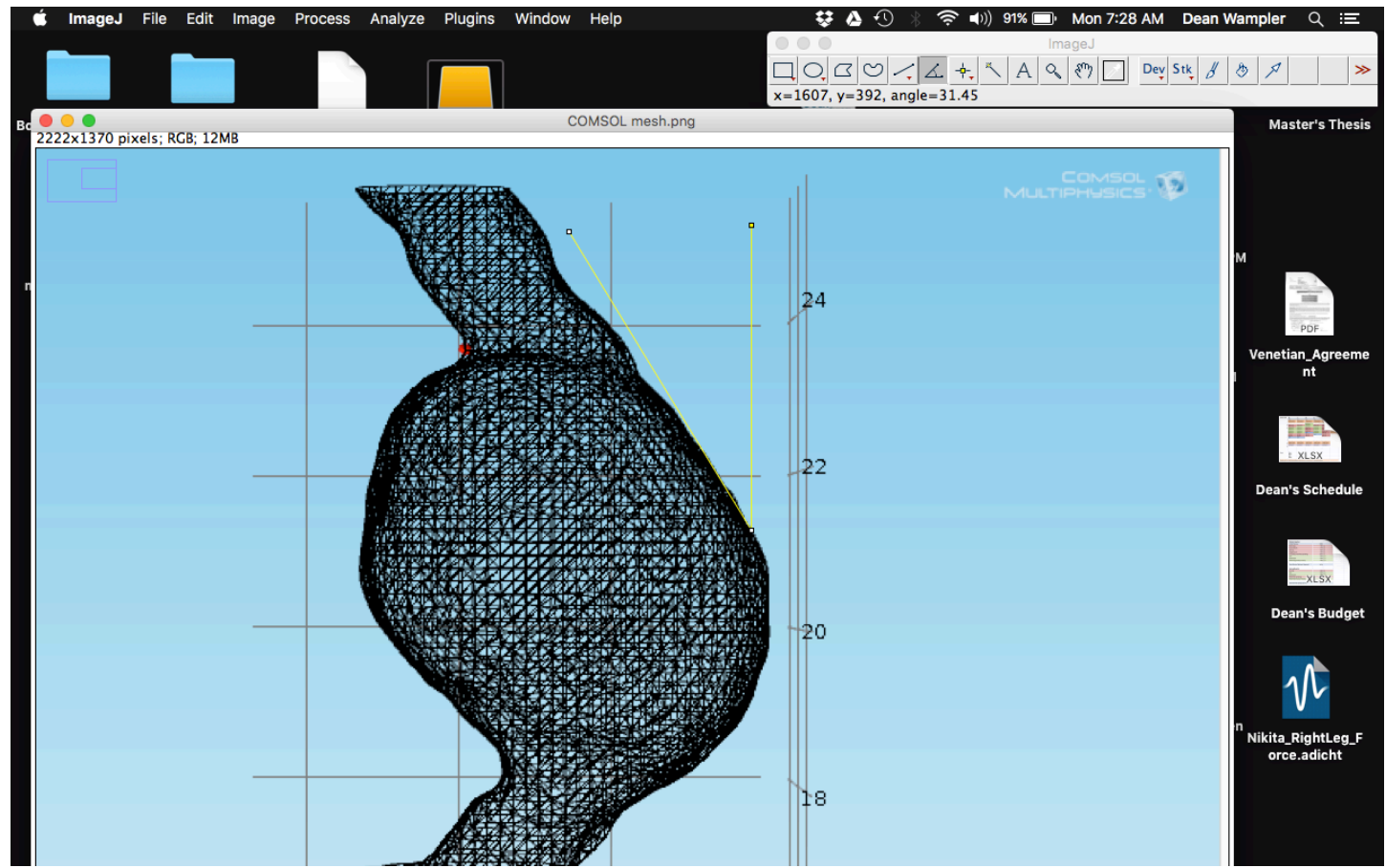

2. Measure the angle of the neck of the aneurysm using the angle tool 
3. Measure the angle of the legs of the aneurysm by using the angle tool

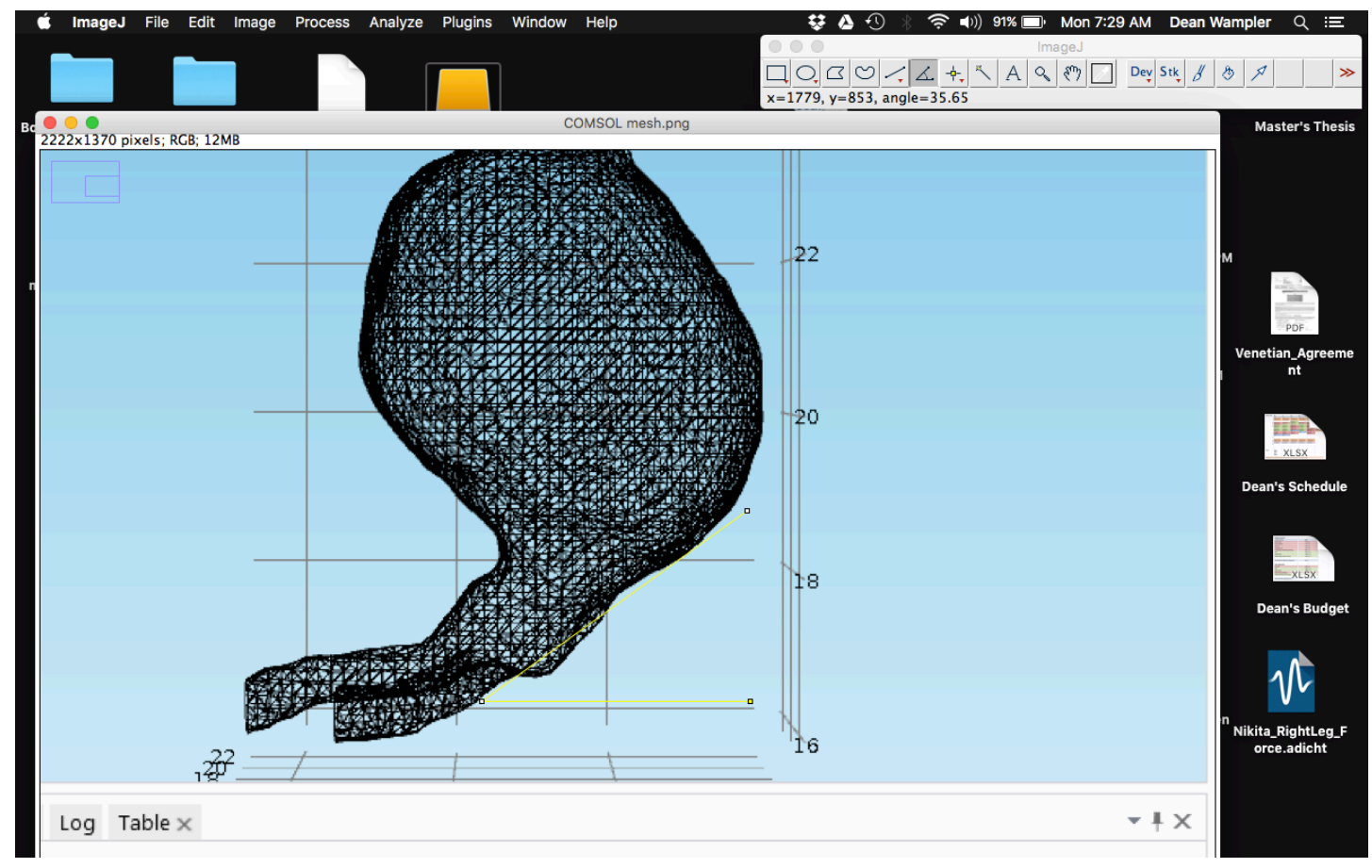

4. Import the image of the PDMS mold into ImageJ.

5. Use the angle measurement tool to measure the angle of the neck of the aneurysm

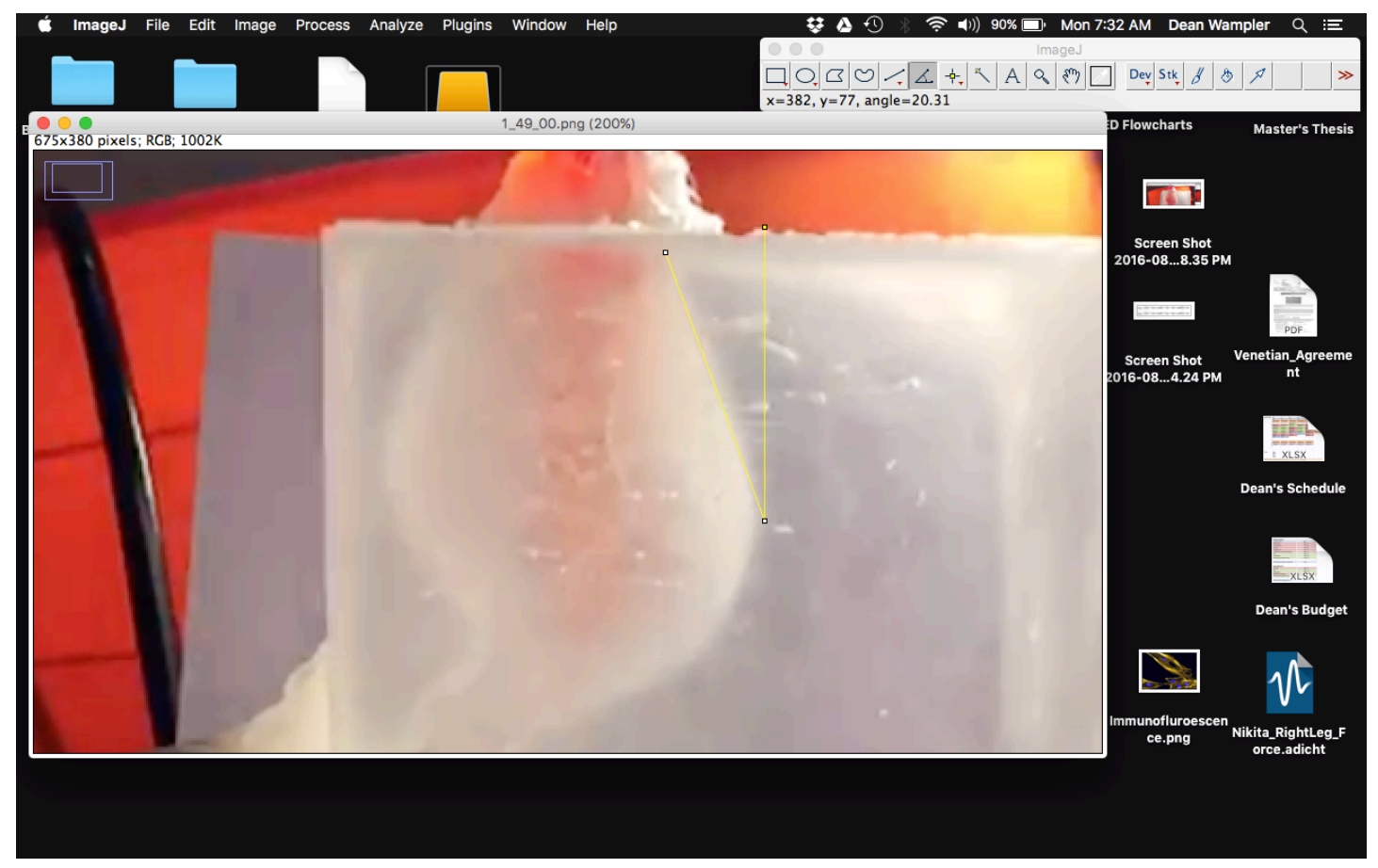


6. Use the angle measurement tool to measure the angle of the legs of the aneurysm

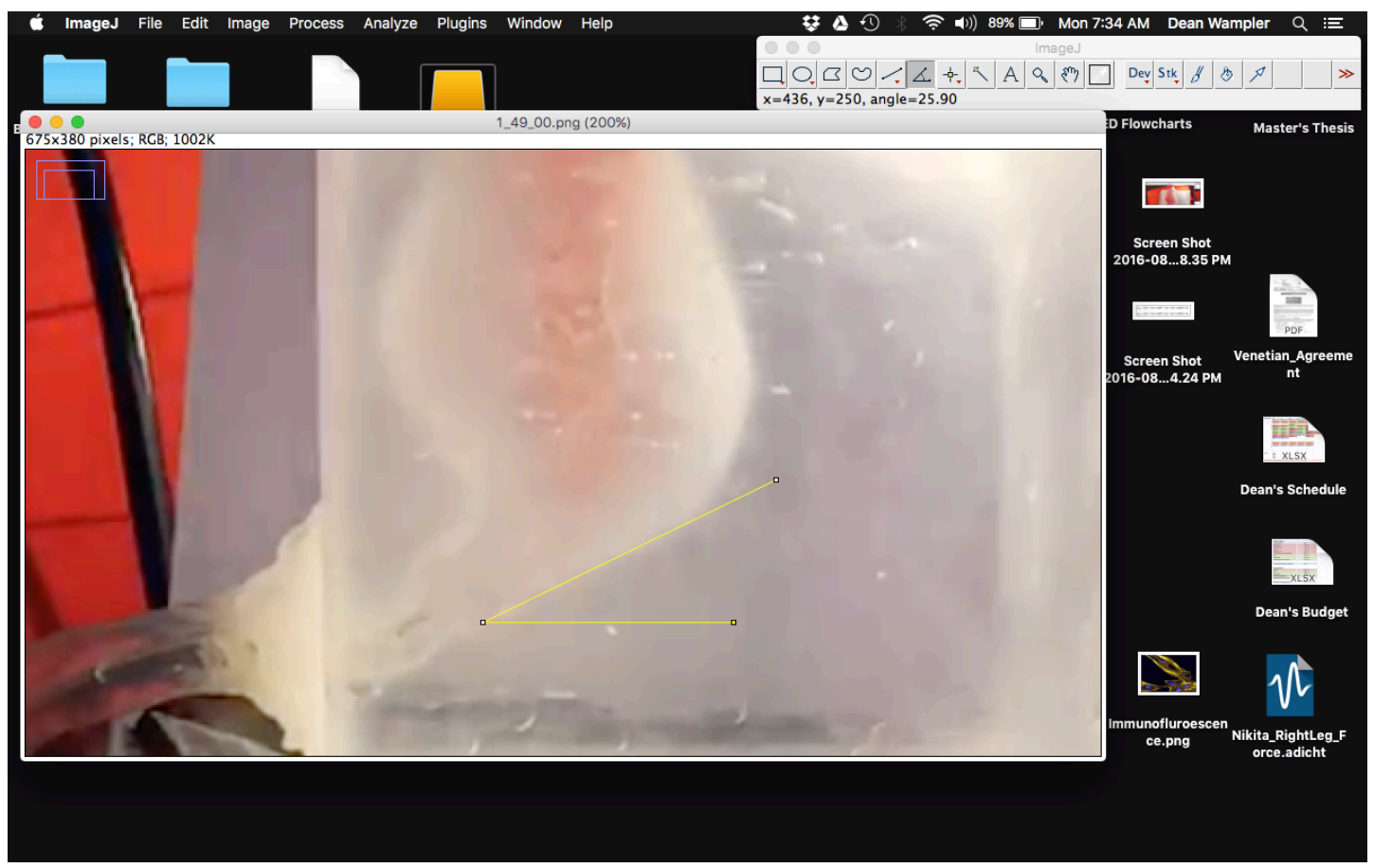

7. To find the angle of rotation of the neck of the aneurysm subtract the angle found from the PDMS mold from the angle found using the COMSOL Multiphysics ${ }^{\circledR}$ model.

$31.45-20.31=11.14$ degrees 
8. To find the angle of rotation of the legs of the aneurysm subtract the angle found from the PDMS mold from the angle found using the COMSOL Multiphysics ${ }^{\circledR}$ model.

$35.65-25.90=9.75$ degrees

9. Find the average between the two angles, and this will be the value of the angle of rotation used in the transformation equation

$(11.14+9.75) / 2=10.445$ degrees 


\section{Appendix I: Coordinate Conversion}

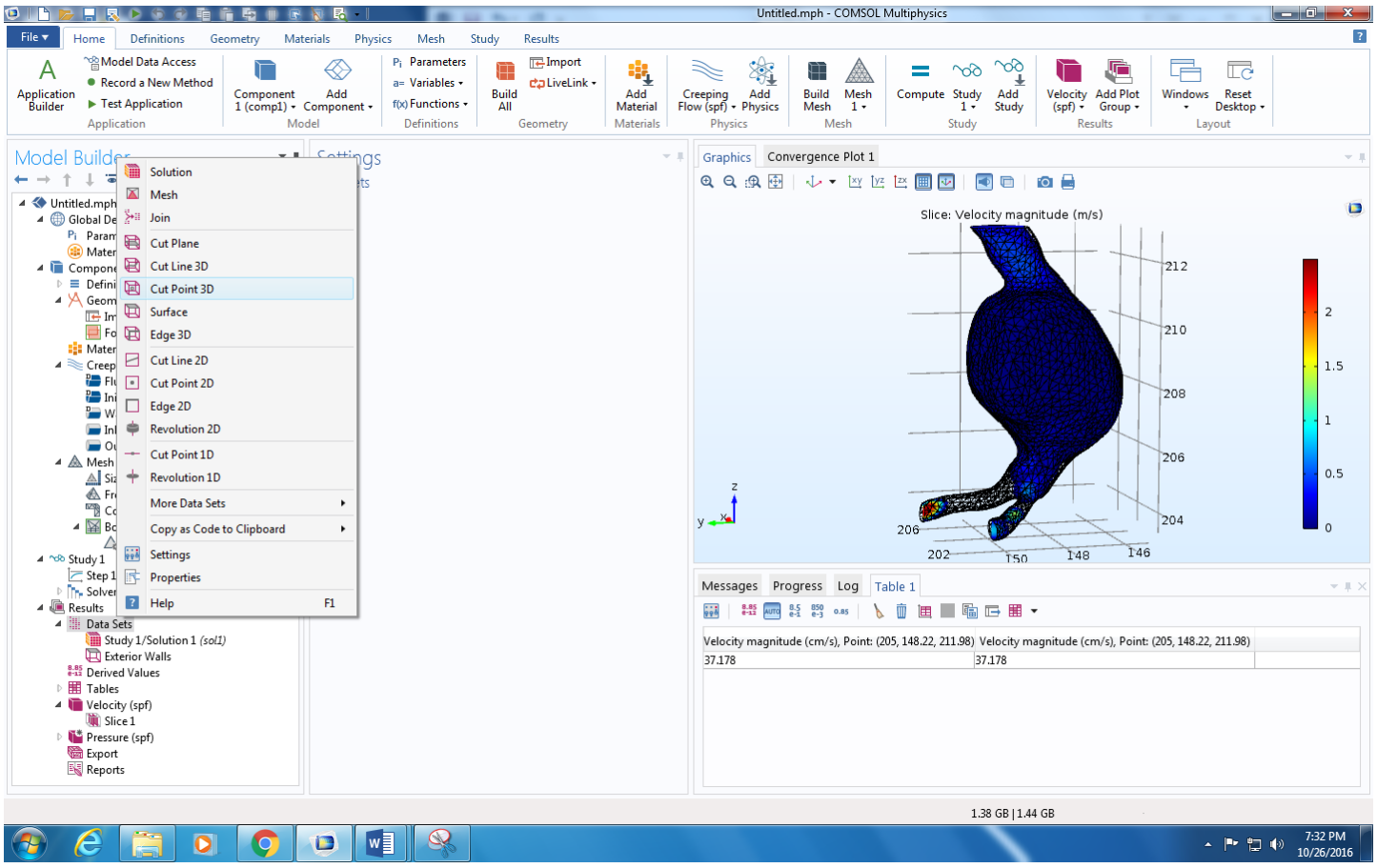

1. Right click Data Sets $>$ Cut Point 3D 
2. Enter the Label "T1a". Enter the $\mathrm{x}, \mathrm{y}$, and $\mathrm{z}$ coordinates associated with the desired point. Click Plot

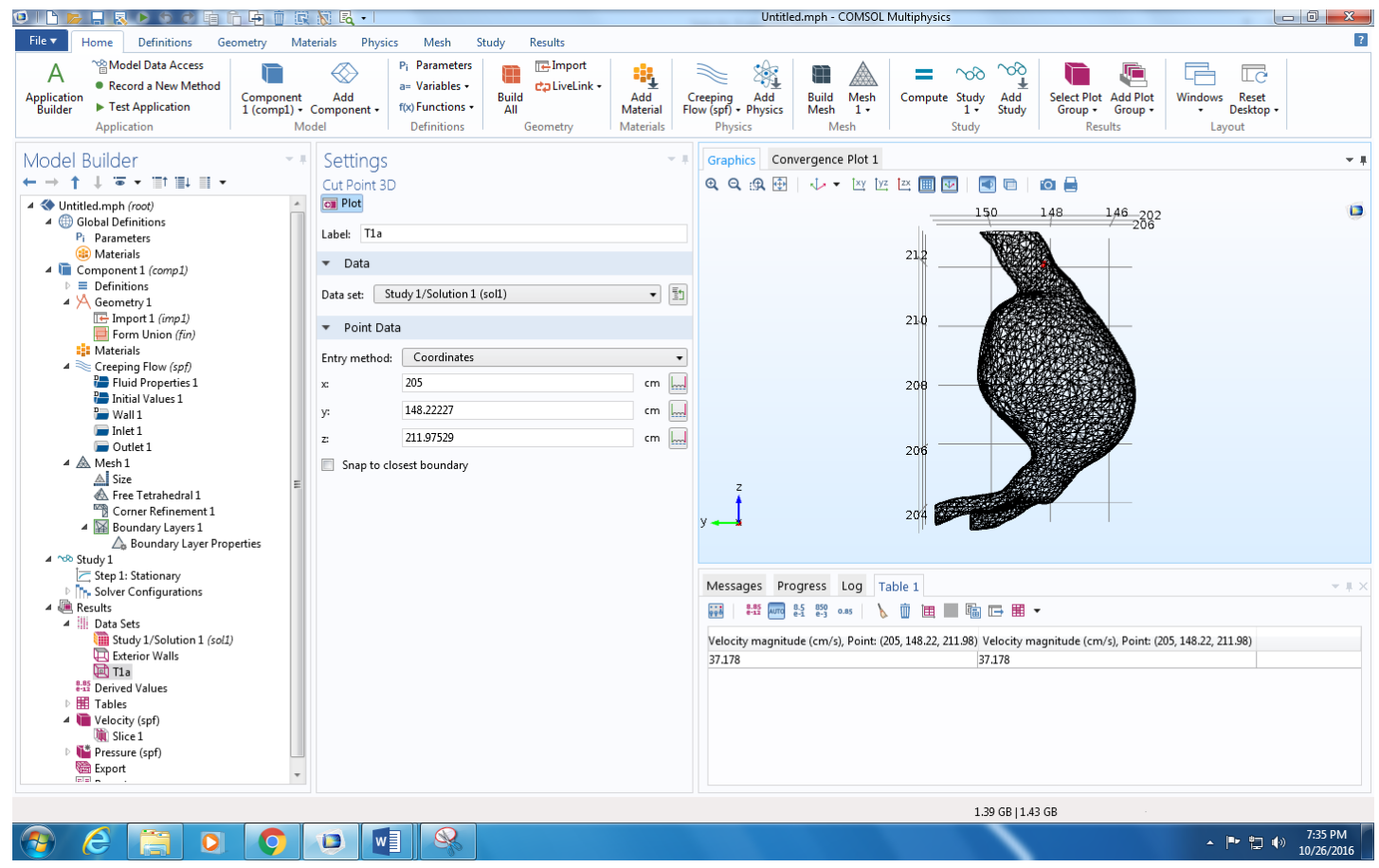


3. Repeat the previous step for all other points to be evaluated.

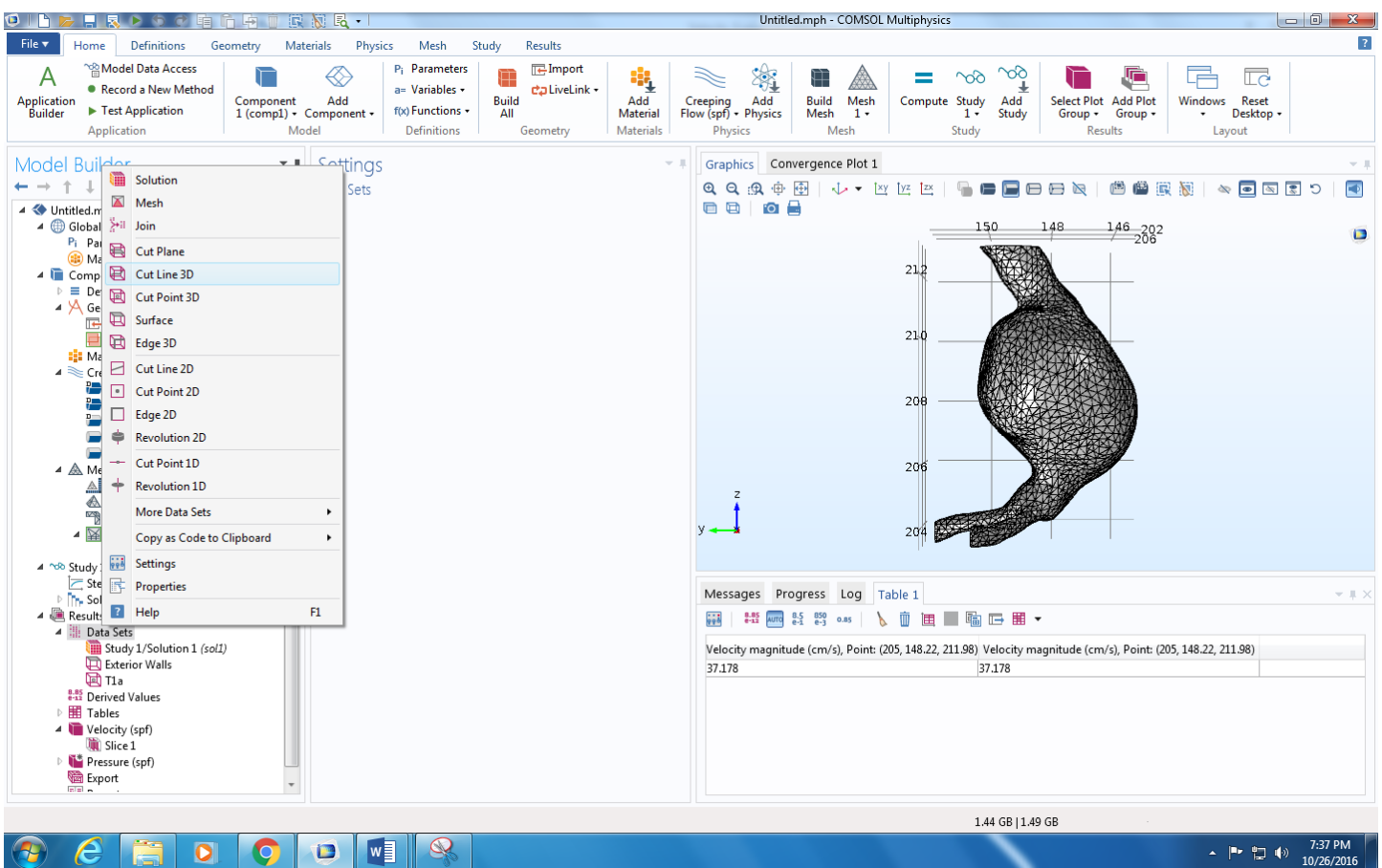

4. Right click on Data Sets $>$ Cut Line 3D 
5. Enter the Label "T1a_line". Enter the y-coordinate from "T1a" in both of the ycoordinate selections. Enter the z-coordinate from "T1a" in both of the zcoordinates. Enter 200 in the x-coordinate for Point 1 and 210 in the x-coordinate for Point 2. This will allow the Cut Line to travel the entire $\mathrm{x}$-distance of the aneurysm.

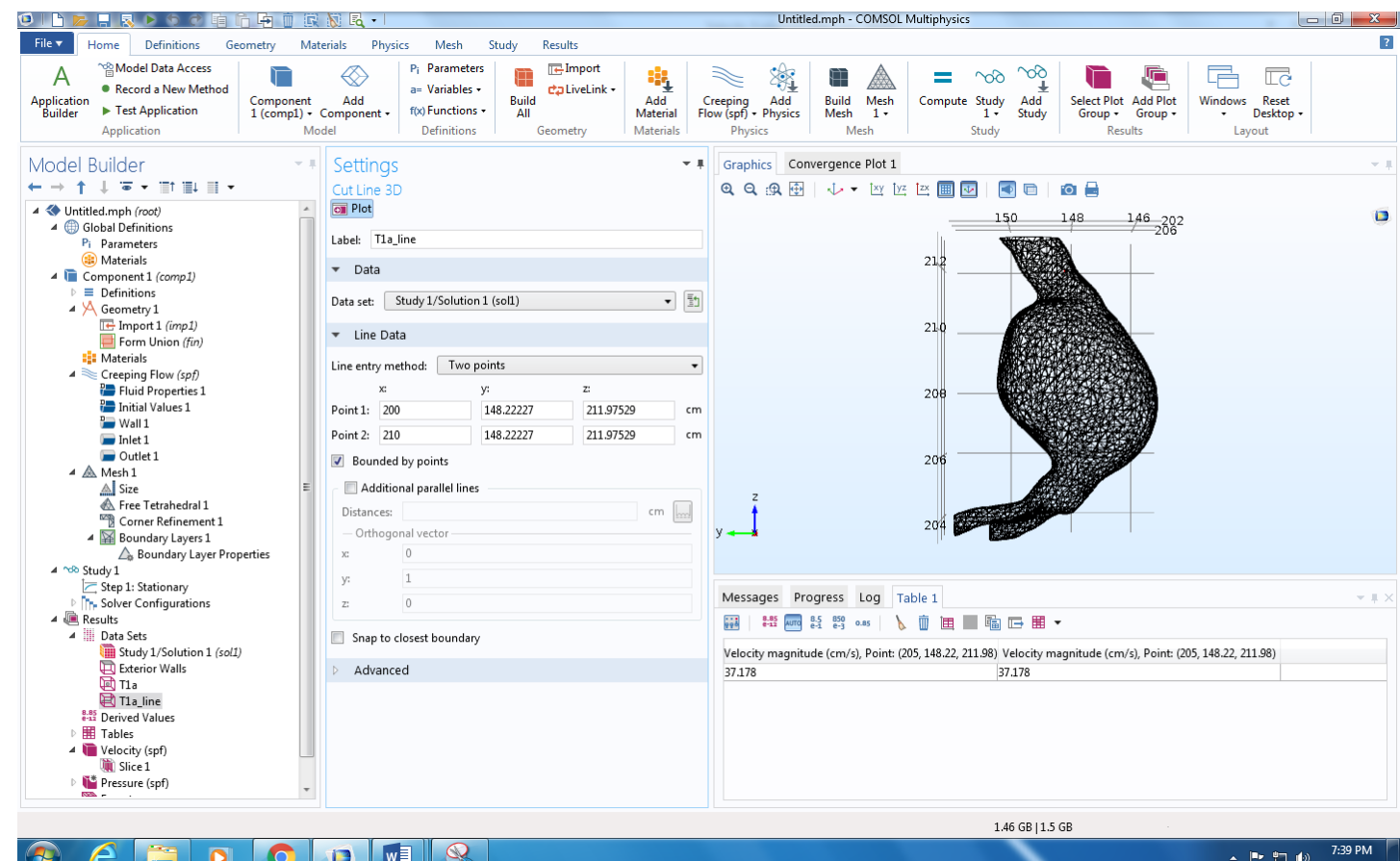

(9) E 
6. Repeat the previous step for all other Line Maximums to be evaluated.

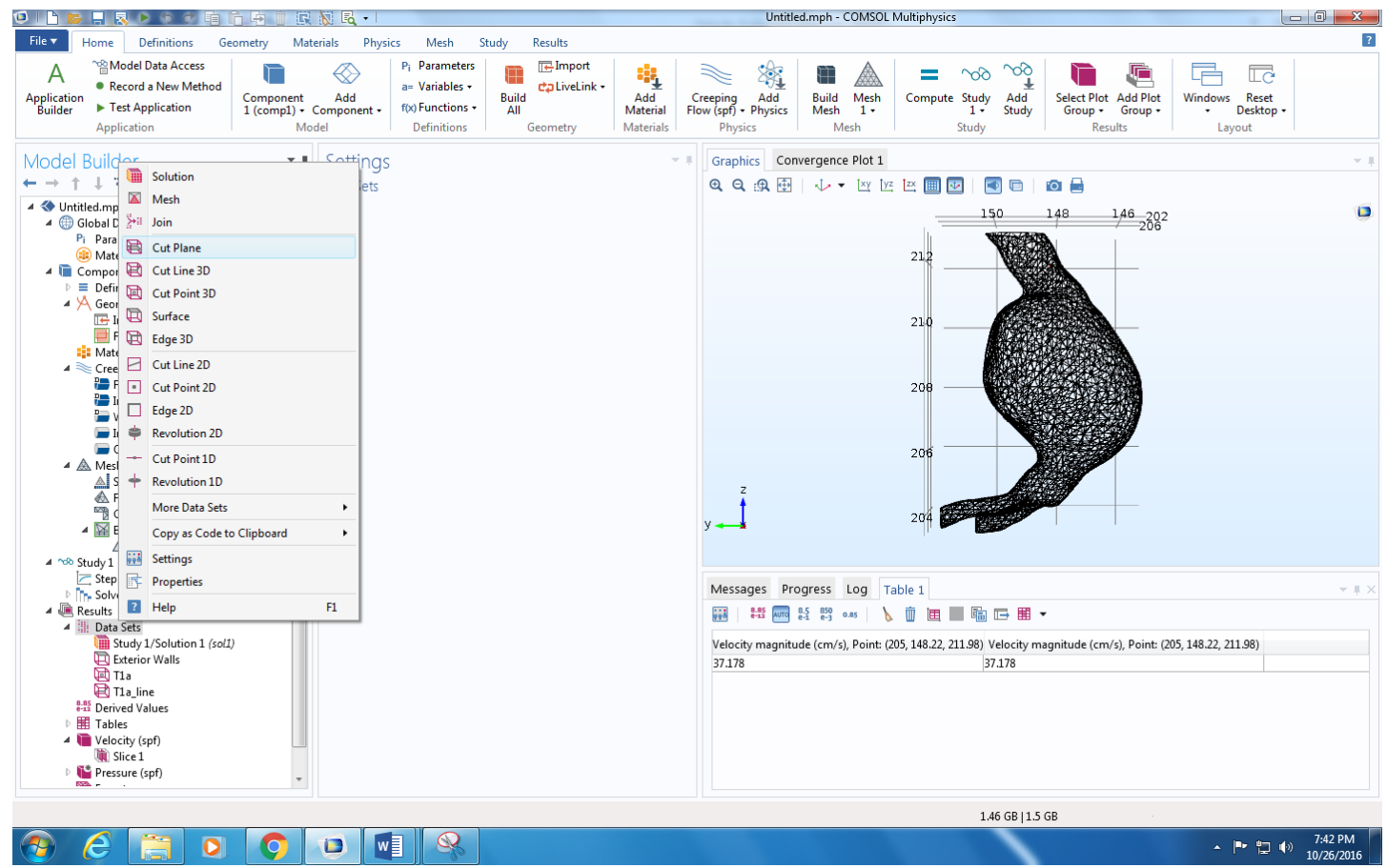


7. Right click on Data Sets $>$ Cut Plane

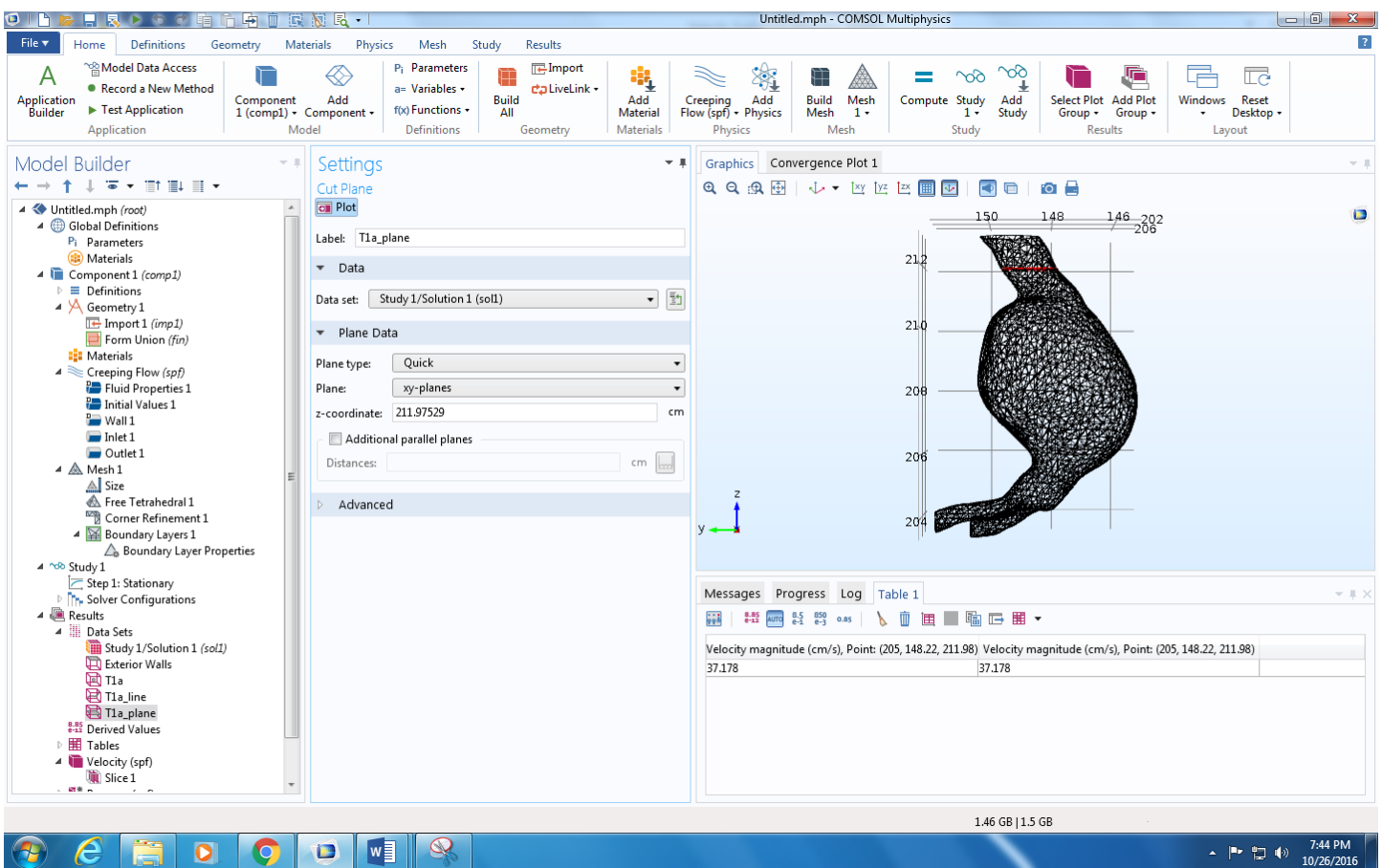

8. Enter the Label "T1a_plane". Under Planes choose xy-planes. Enter the zcoordinate from "T1a"

9. Repeat the previous step for all other Plane Maximums to be evaluated. 


\section{Right Click on Derived Values $>$ Point Evaluation}

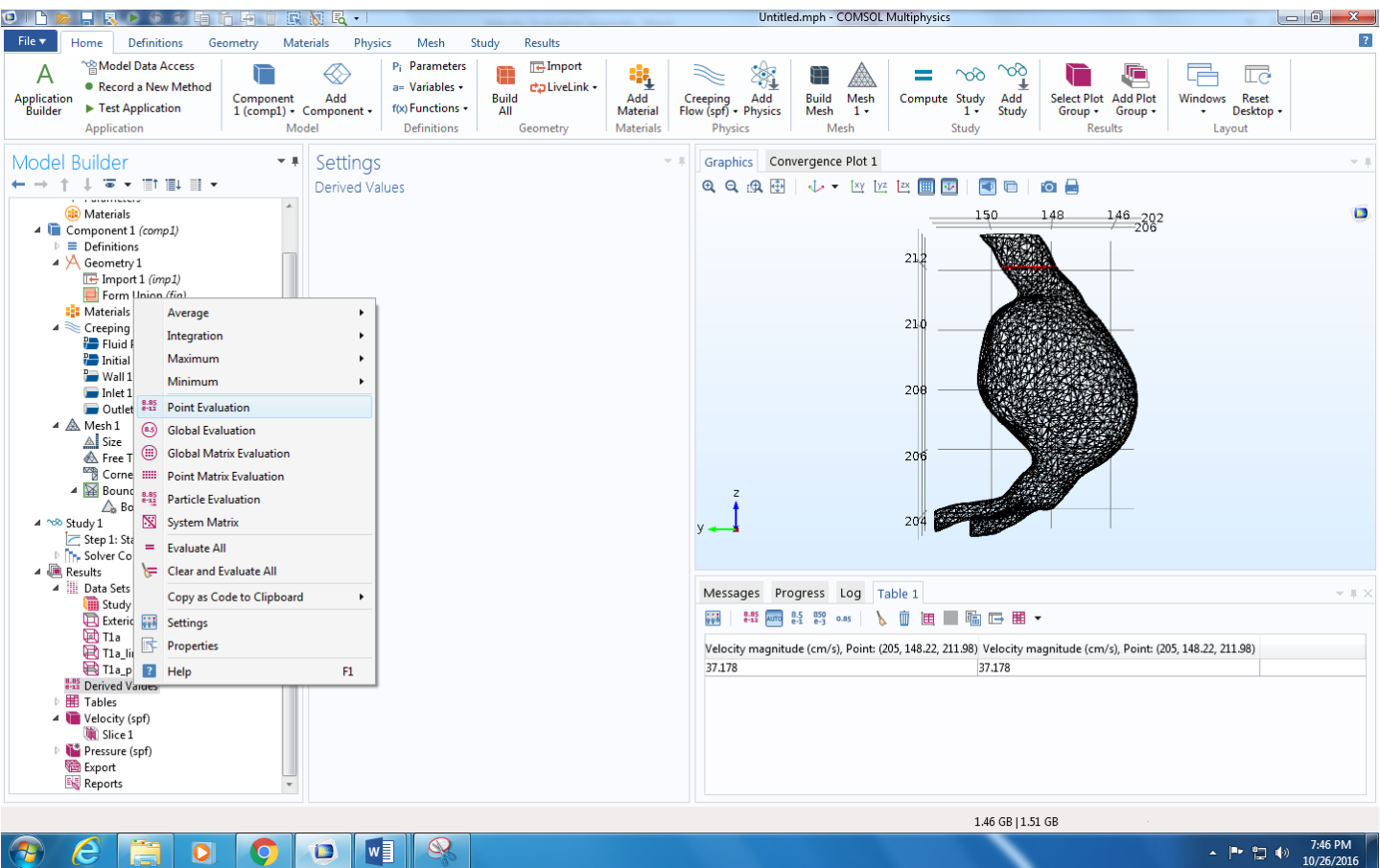


11. Select Point Evaluation. Under Data Set choose "T1a". Under Expression choose “spf.U”. Change Unit to cm/s. Select Evaluate.

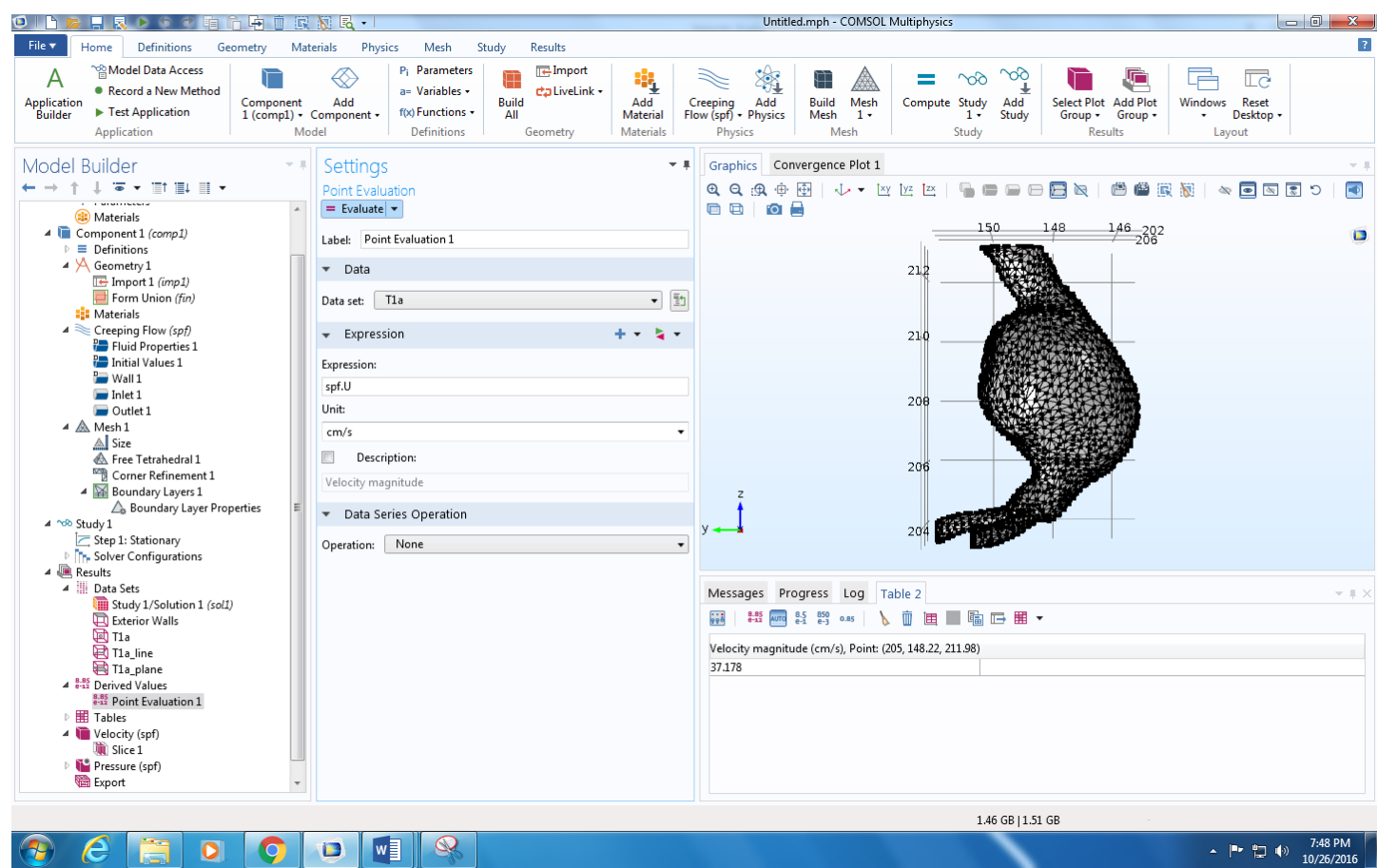

12. Repeat the previous step for all other points to be evaluated. 


\section{Right Click on Derived Values $>$ Maximum $>$ Line Maximum}

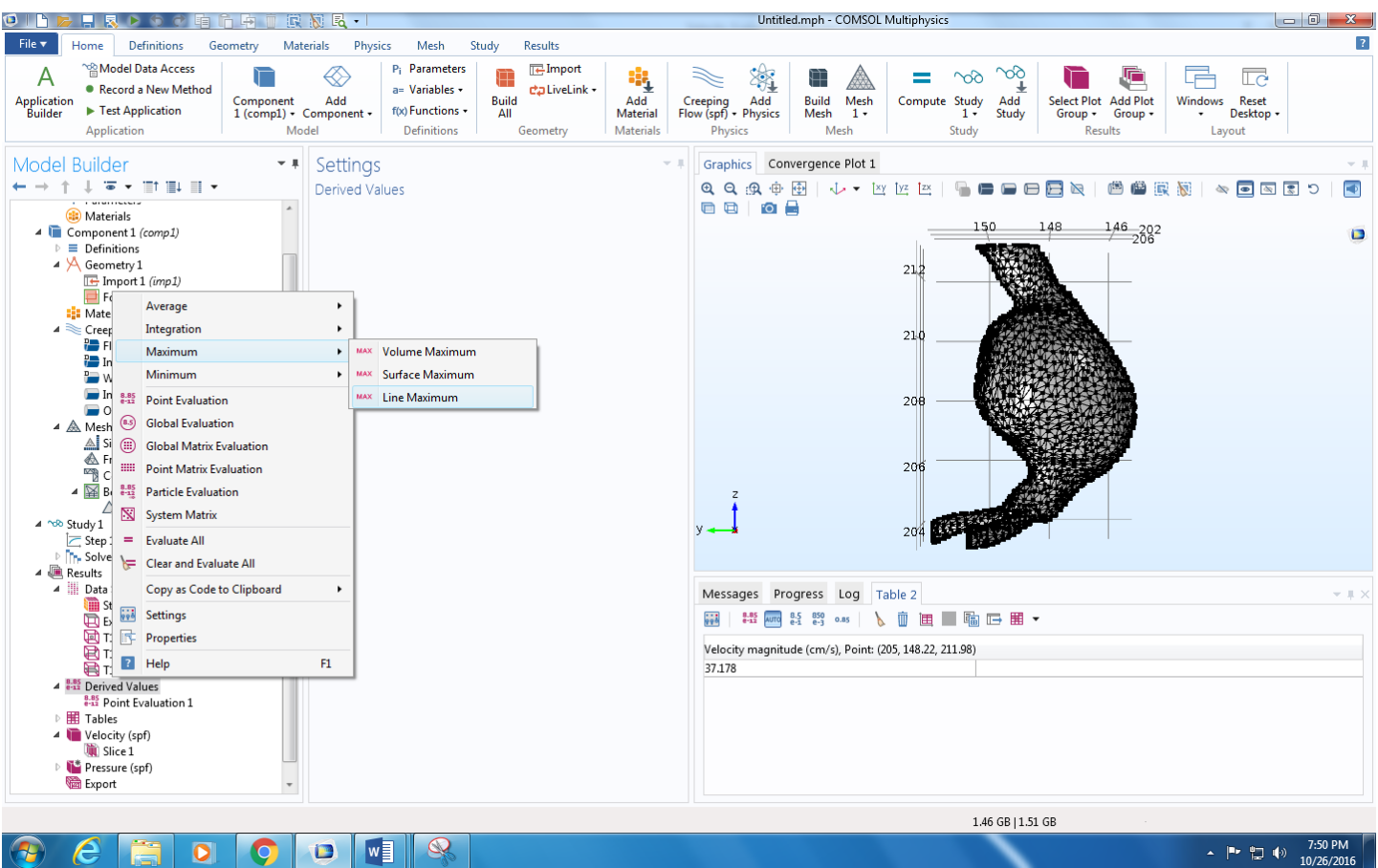


14. Select Line Maximum. Under Data Set, choose T1a_line. For Expression, enter "spf.U”. For Unit, choose cm/s. Click Evaluate.

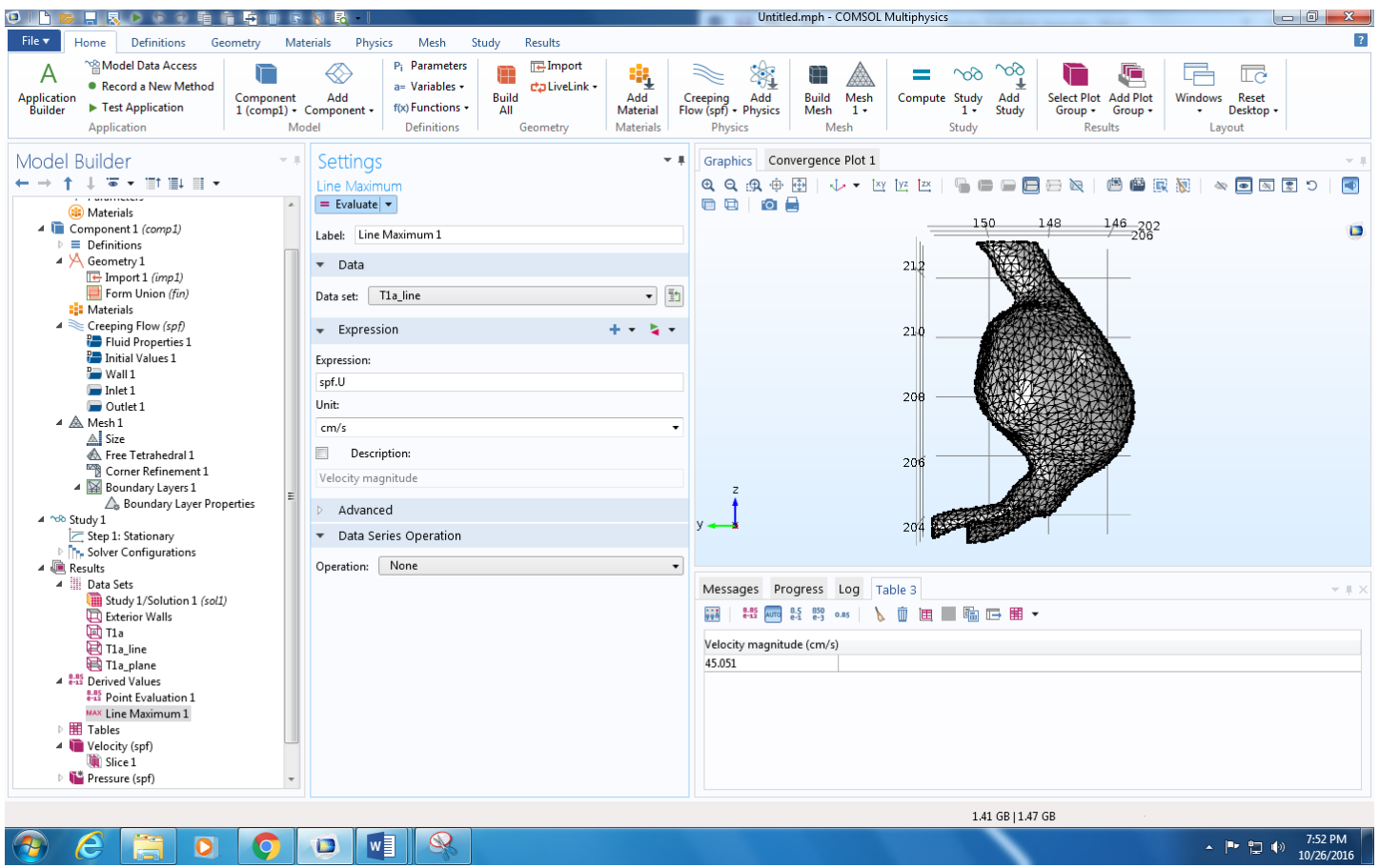


15. Repeat the previous step for all other Line Maximums to be evaluated.

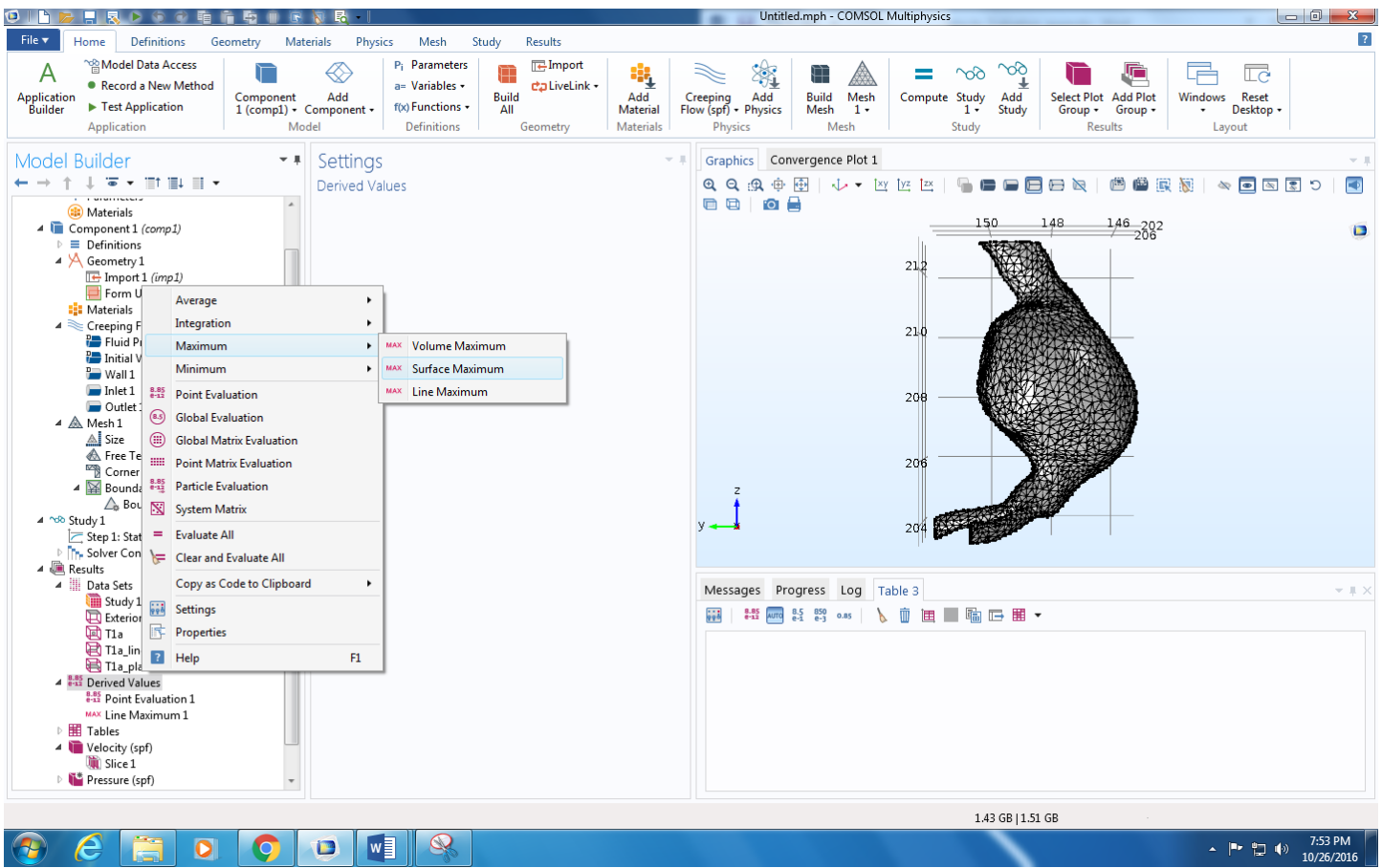

16. Right Click Derived Values $>$ Surface Maximum 
17. Select Surface Maximum. Under Data Set Select T1a_plane. For Expression, enter "spf.U”. For Unit, choose cm/s. Click Evaluate.

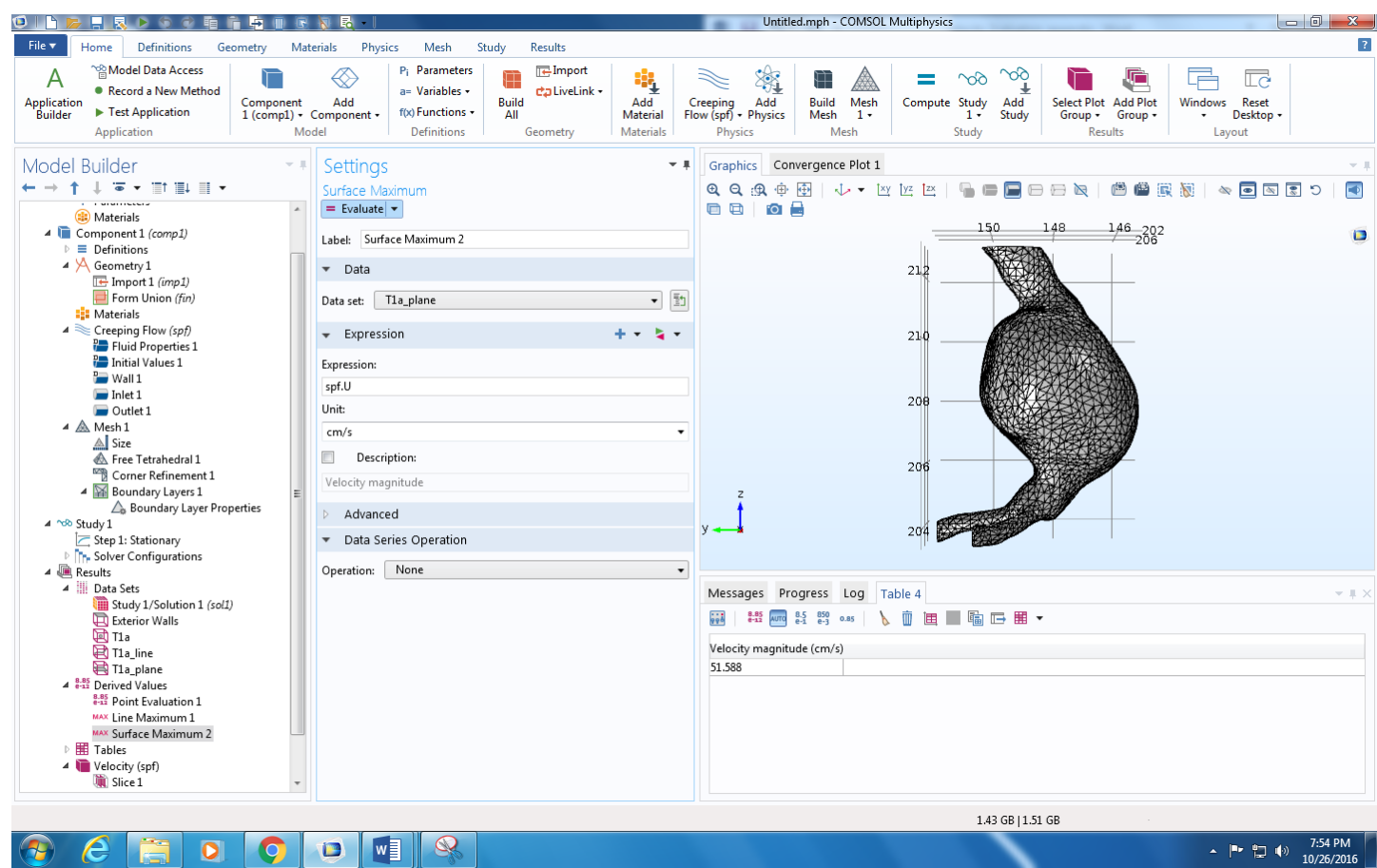

18. Repeat the previous step for all other Plane Maximums to be evaluated. 


\section{Appendix J: Mesh Confidence Study}

1. Choose point to be evaluated at different Mesh levels (T3b)

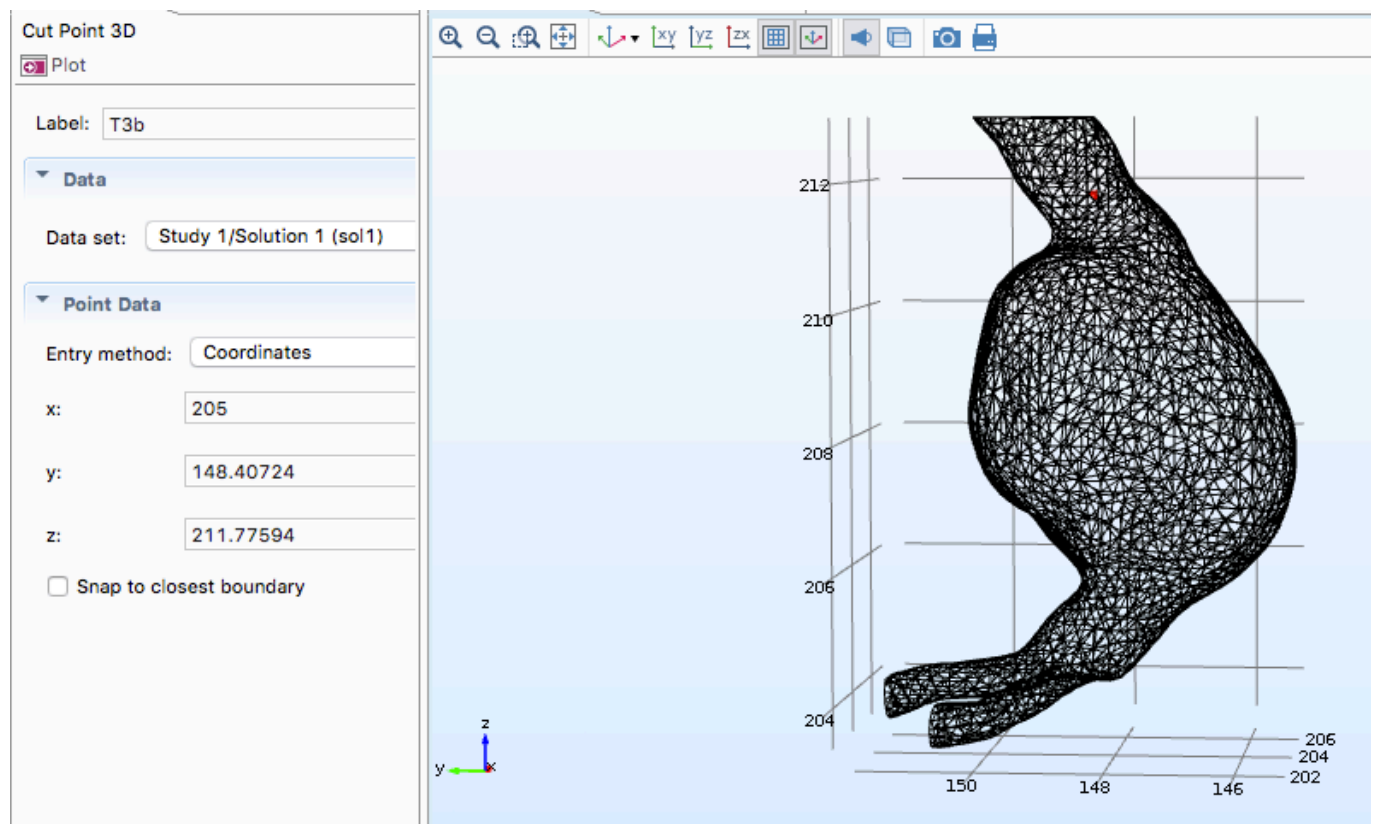

2. Evaluate the maximum velocity at that point

\begin{tabular}{|c|c|}
\hline & Point \\
\hline Predefined Mesh & Maximum Velocity $(\mathrm{cm} / \mathrm{s})$ \\
\hline Normal & 53.159 \\
\hline Fine & 58.572 \\
\hline Finer & 60.063 \\
\hline Extra Fine & N/A \\
\hline Extremely Fine & N/A \\
\hline
\end{tabular}

3. Compare the velocity at that mesh level with the value at the lower level

4. Stop when there is less than a $5 \%$ difference in velocity

\begin{tabular}{|l|r|}
\hline Percent Difference & \\
\hline Predefined Mesh & \\
\hline Normal to Fine & 10.18265957 \\
\hline Fine to Finer & 2.545584921 \\
\hline Finer to Extra Fine & \\
\hline
\end{tabular}




\section{Appendix K: COMSOL Multiphysics ${ }^{\circledR}$ Setup}

1. Open a blank model

2. Right Click Untitled.mph $>$ Add Component $>3 D$

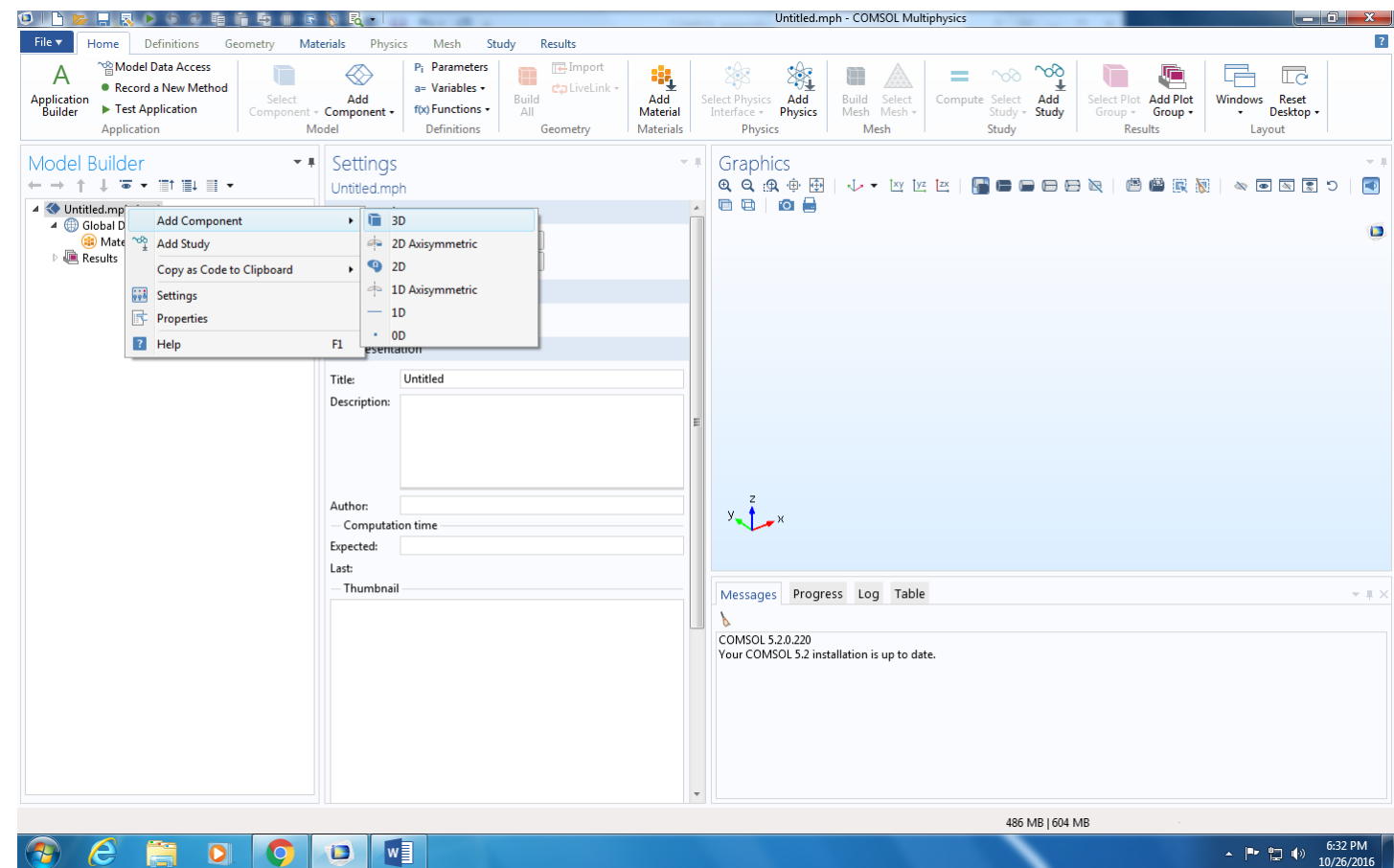


3. Right Click Global Definitions $>$ Parameters

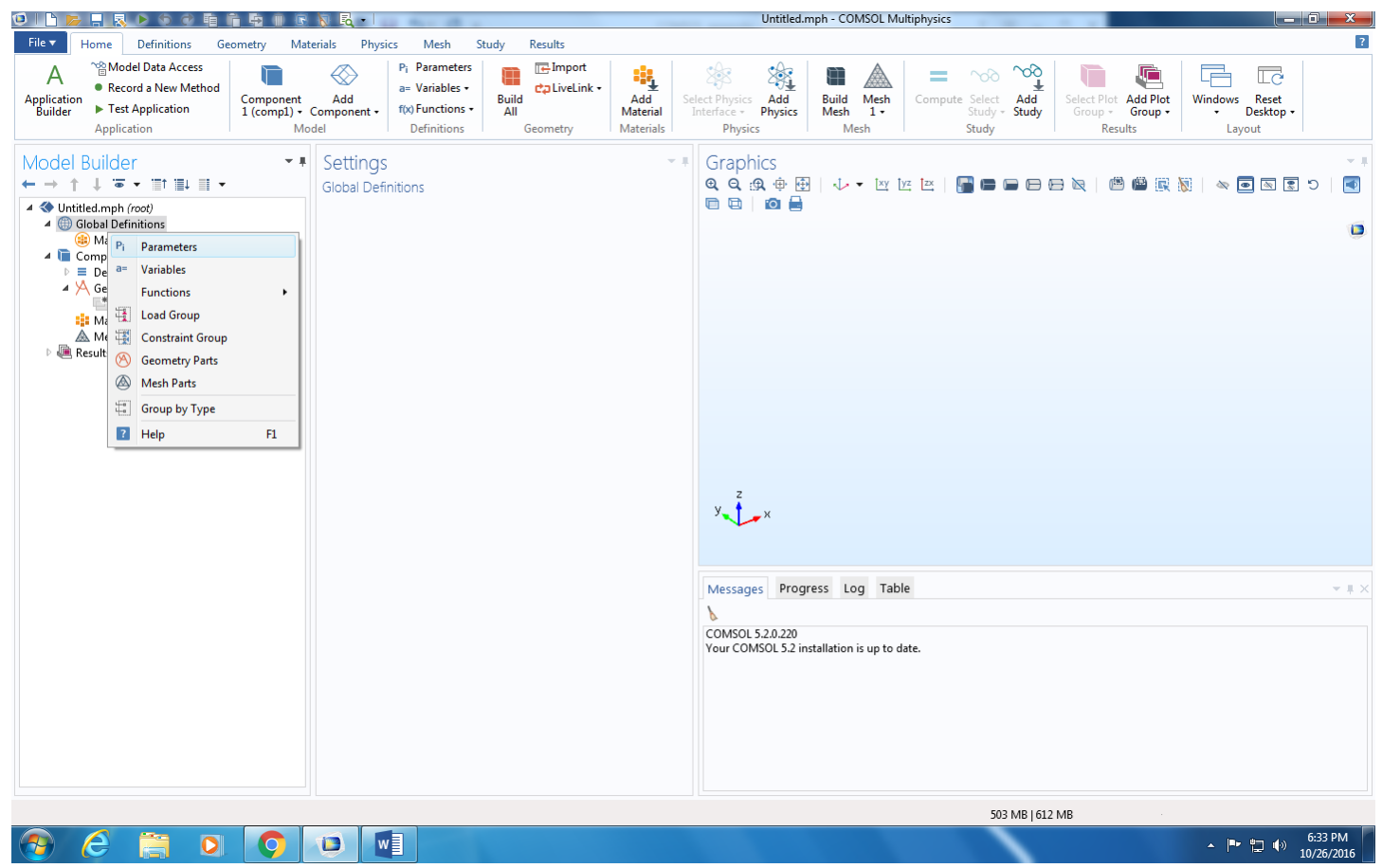

4. Input the following parameters:

- Parameters

\begin{tabular}{|l|l|l|l|}
\hline Name & Expression & Value & Description \\
\hline P_out & $1[$ atm] & $1.0133 E 5 \mathrm{~Pa}$ & \\
rho & $1000\left[\mathrm{~kg} / \mathrm{m}^{\wedge} 3\right]$ & $1000 \mathrm{~kg} / \mathrm{m}^{3}$ & \\
vis & $.89[\mathrm{cP}]$ & $8.9 \mathrm{E}-4 \mathrm{~Pa} \cdot \mathrm{s}$ \\
\hline Q_in & $(1 / 15.094)[\mathrm{L} / \mathrm{s}]$ & $6.6251 \mathrm{E}-5 \mathrm{~m}^{3} / \mathrm{s}$ & \\
mdotin & rho*Q_in & $0.066251 \mathrm{~kg} / \mathrm{s}$ & \\
\hline
\end{tabular}


5. Right click Geometry $1>$ Import. Change length unit to $\mathrm{cm}$

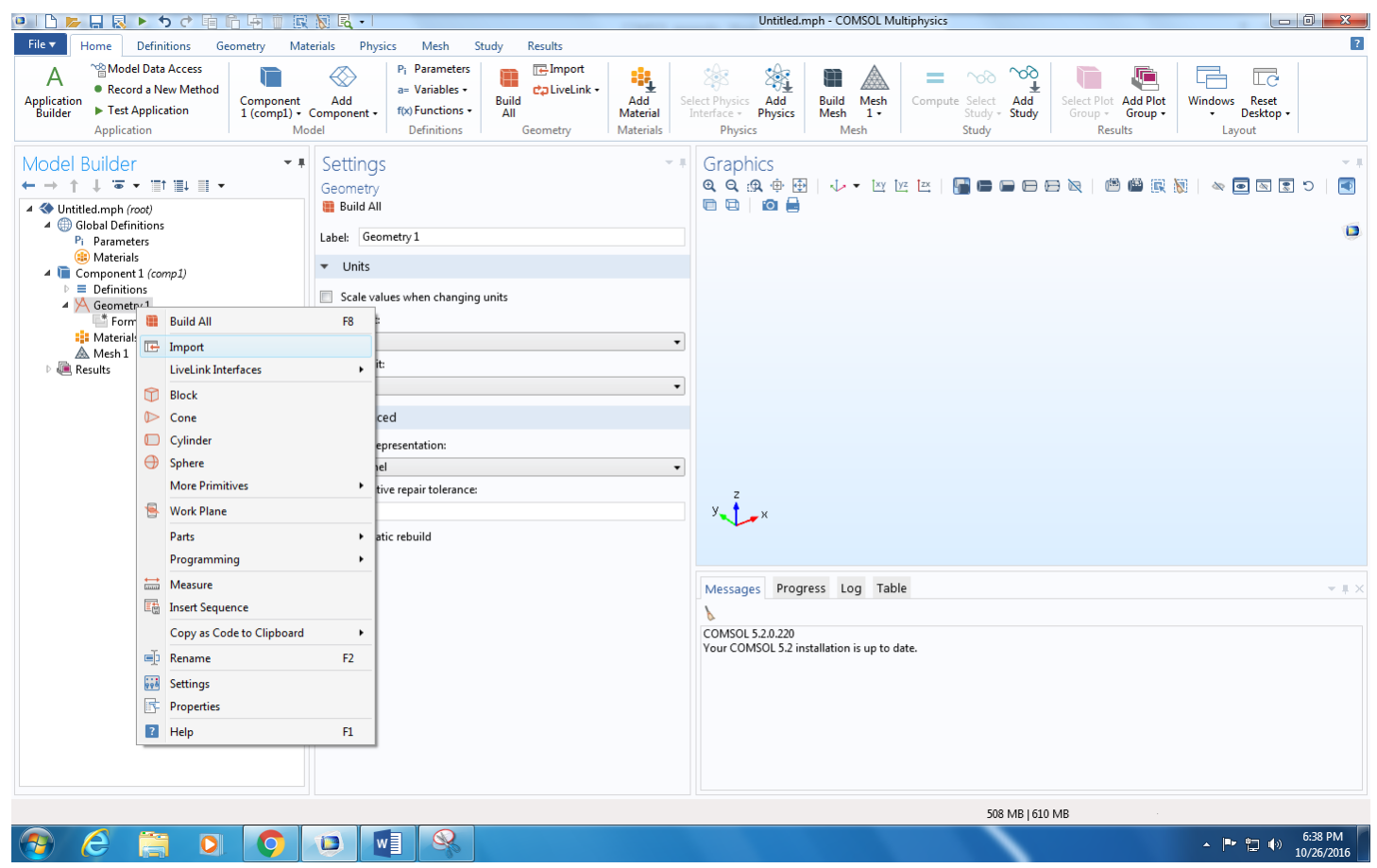




\section{Import .IGS file}

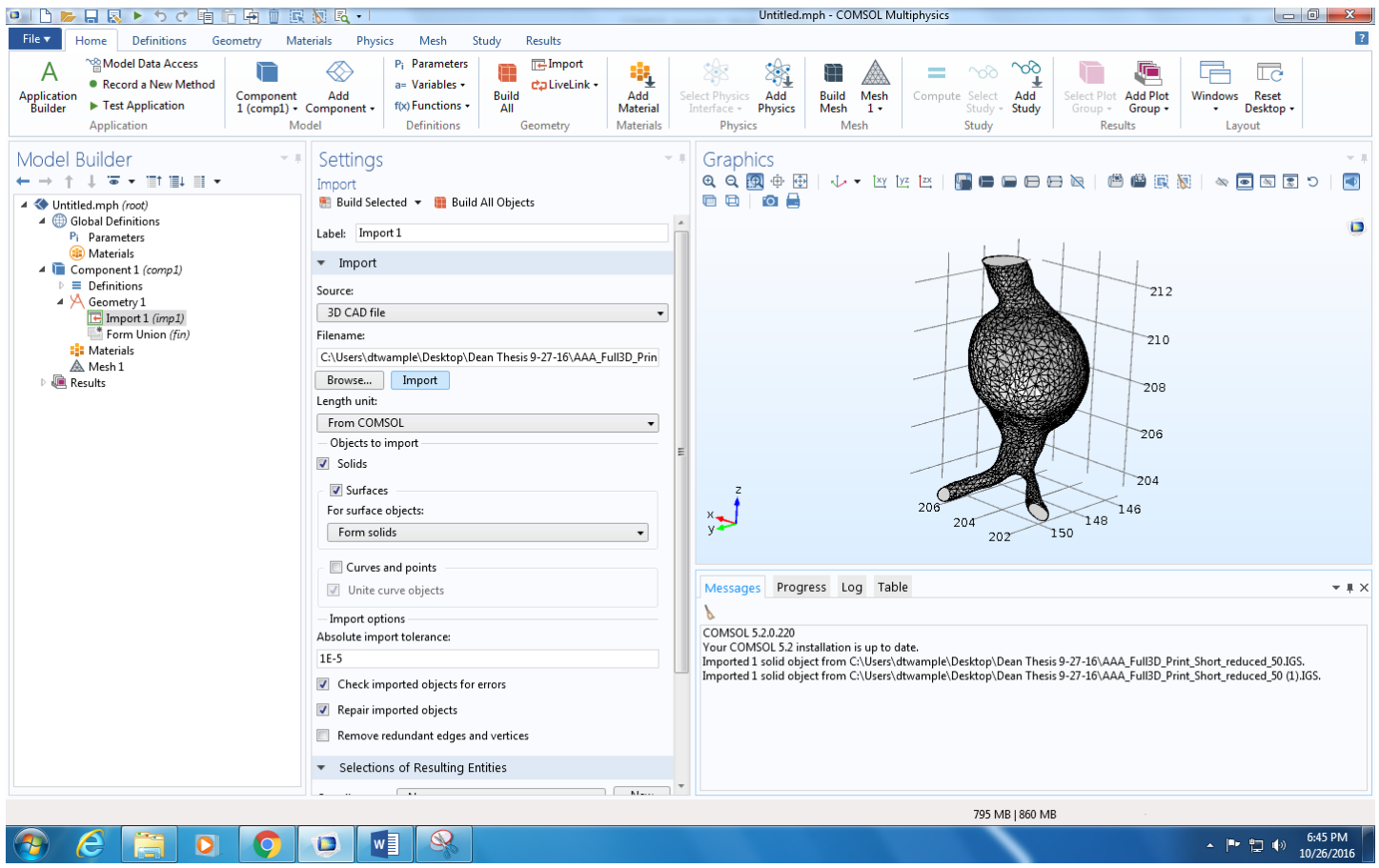




\section{Physics $>$ Add Physics}

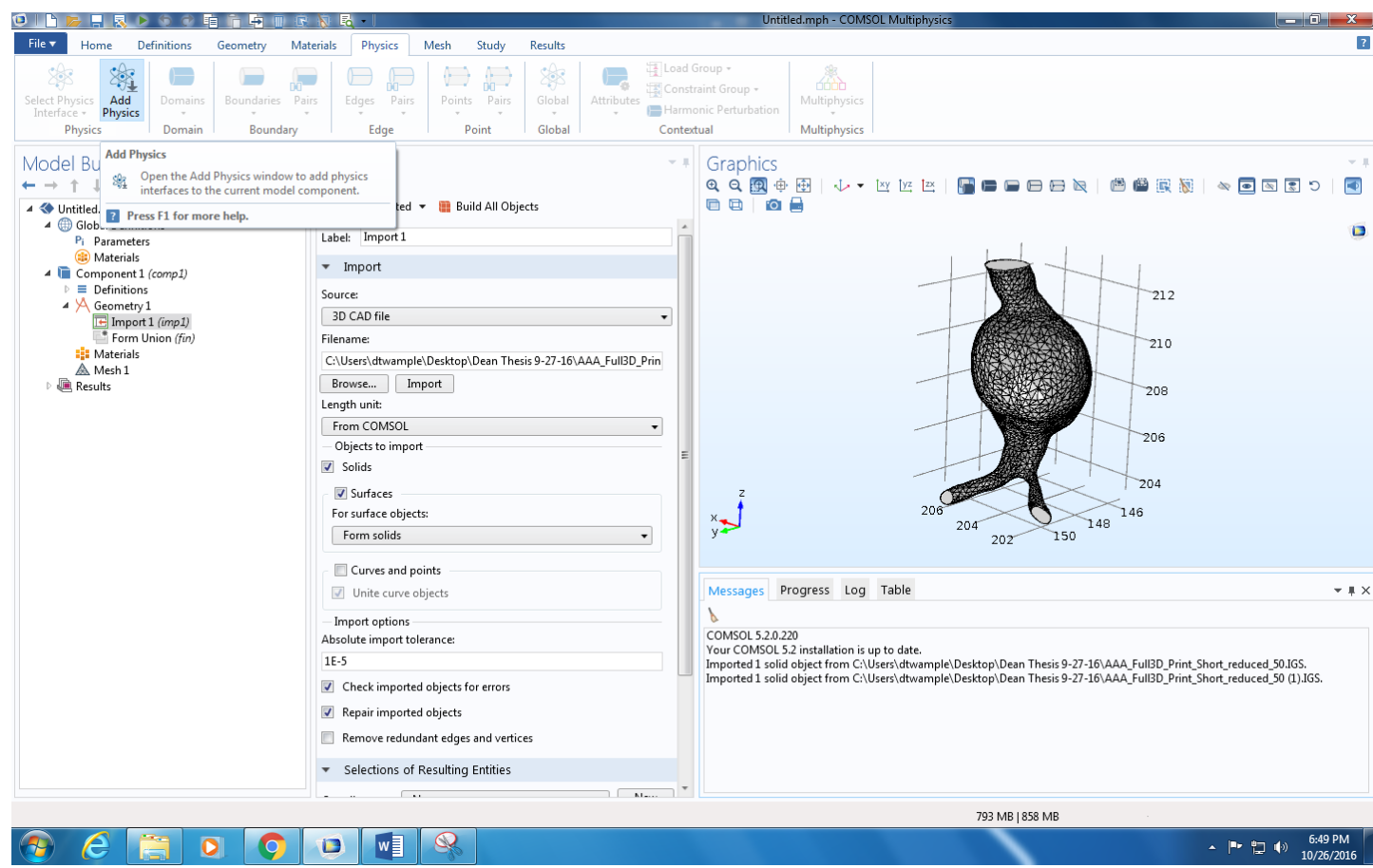




\section{Choose Laminar Flow and Add to Component}

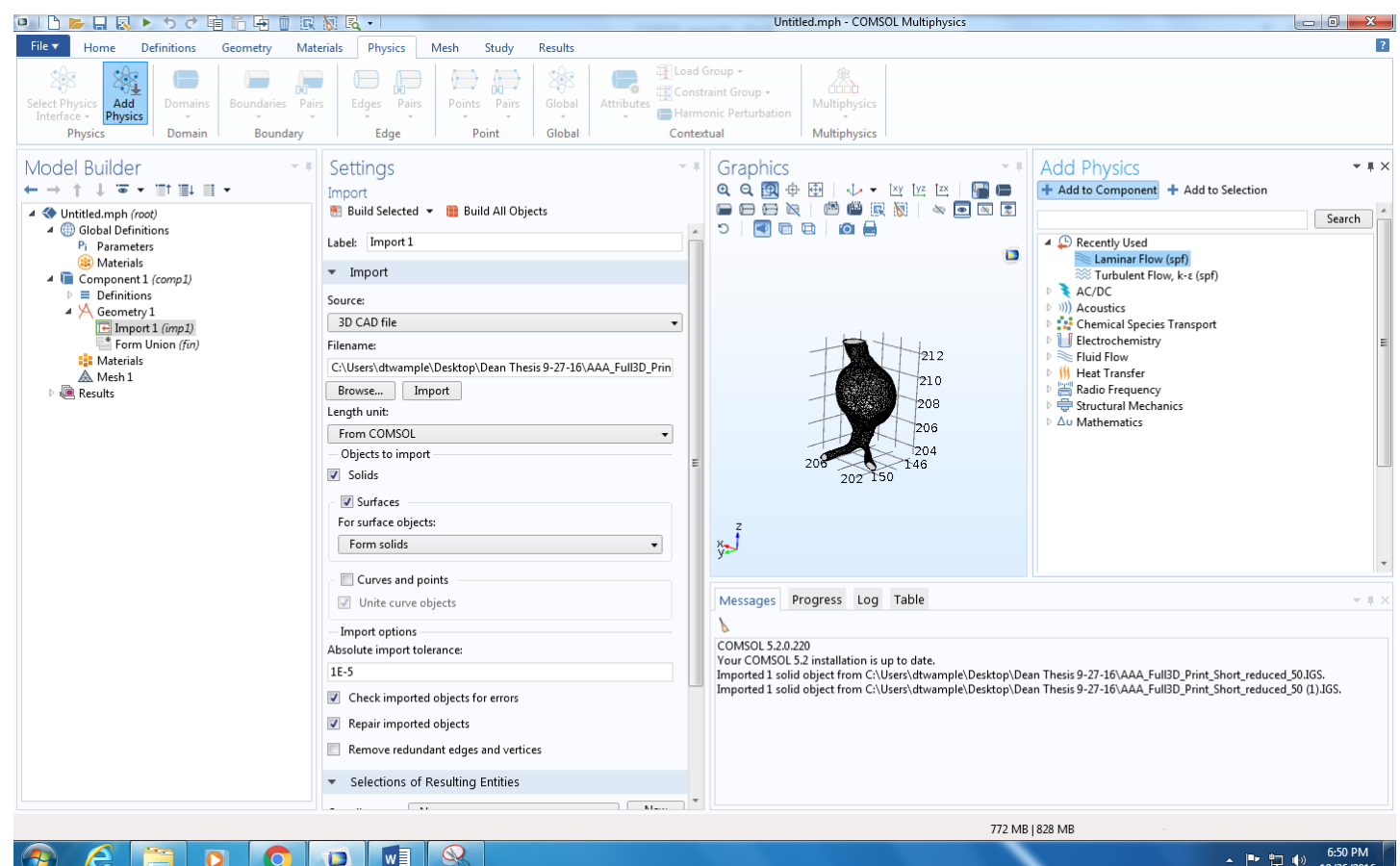


9. Click Fluid Properties. Choose user defined for density and dynamic viscosity.

Enter "rho" for density, and "vis" for dynamic viscosity.

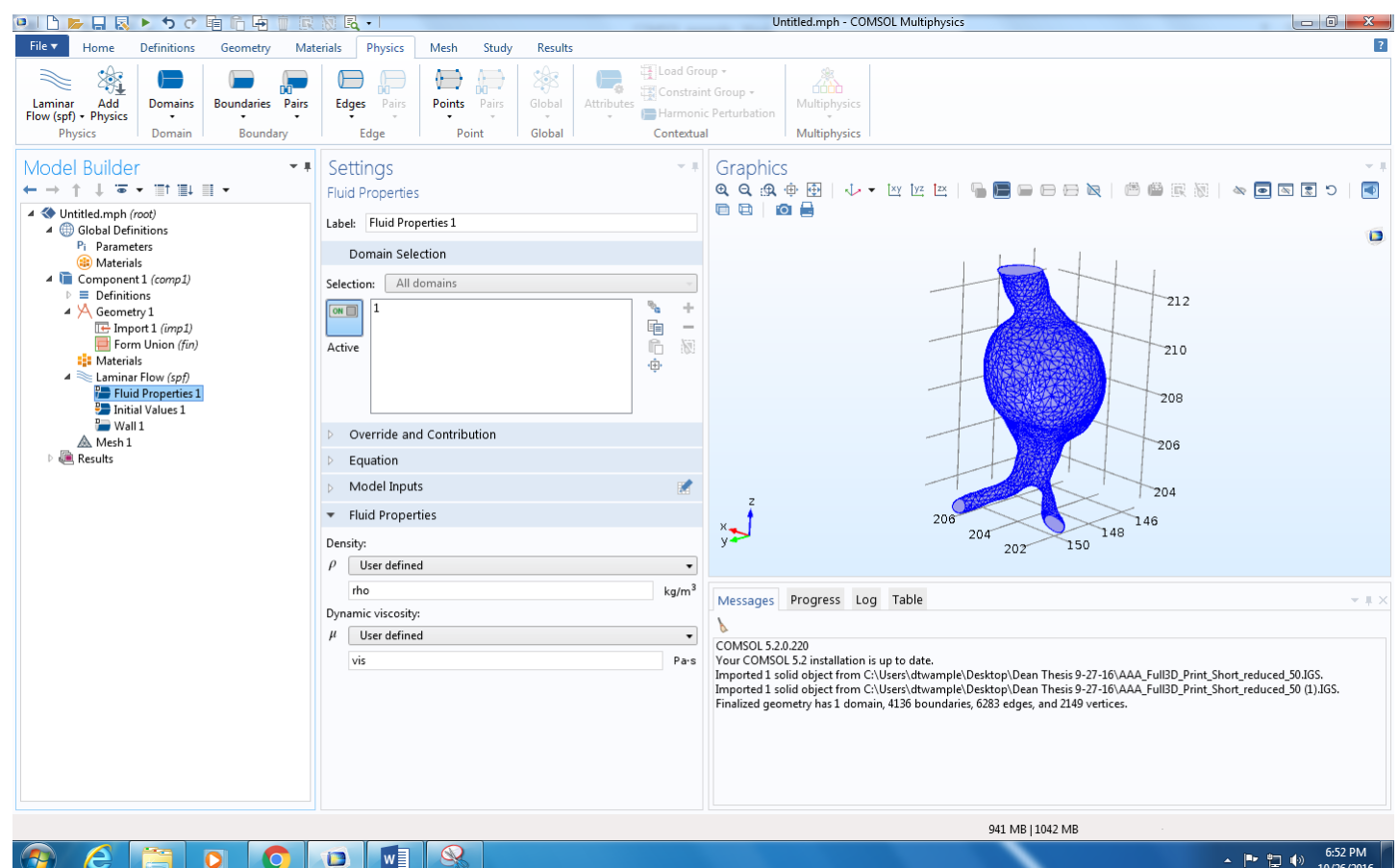




\section{Right click Laminar Flow > Inlet}

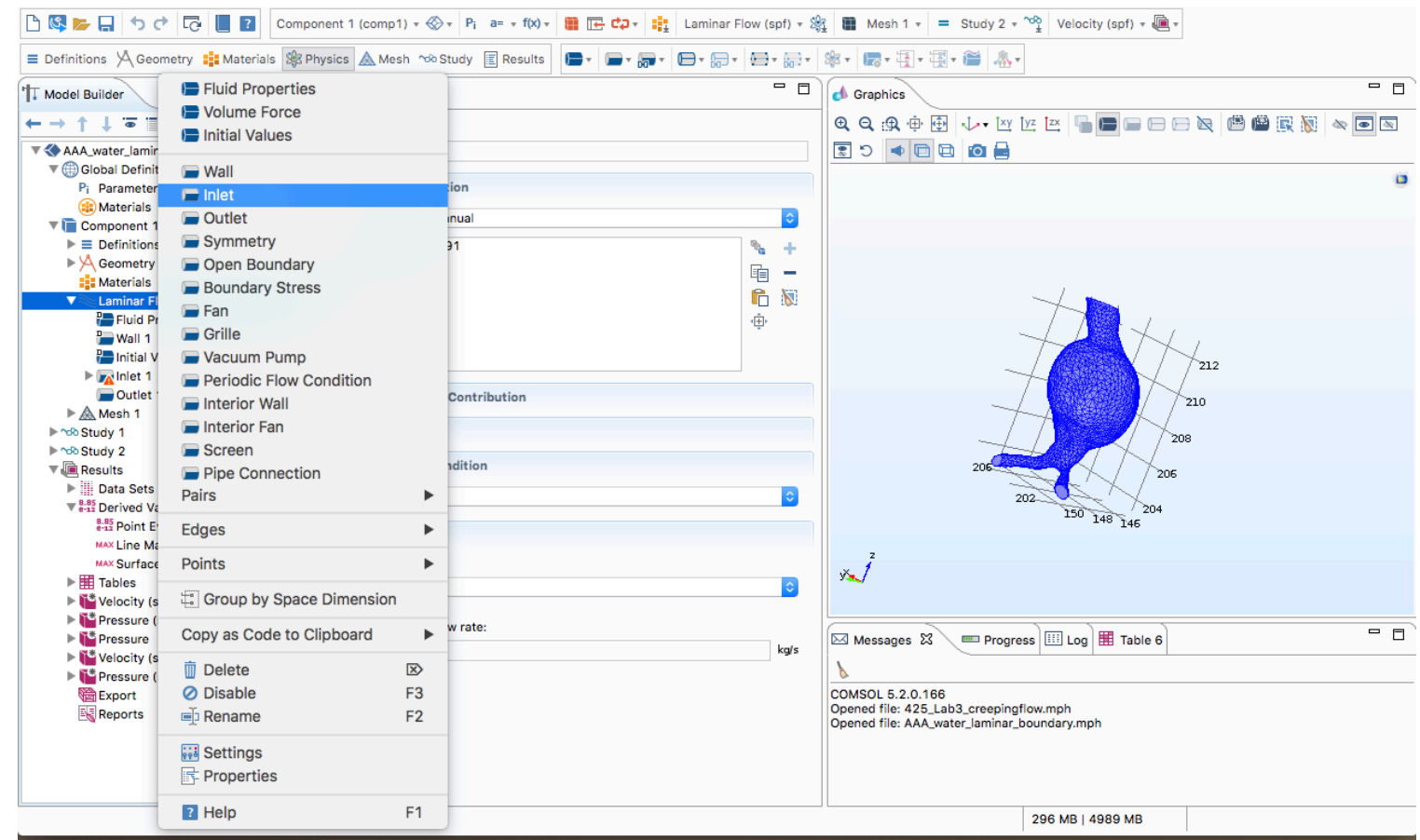


11. Under Boundary Conditions select Mass Flow. Enter "mdotin" for the normal mass flow rate. Choose the Inlet as the top of the neck of the aneurysm.

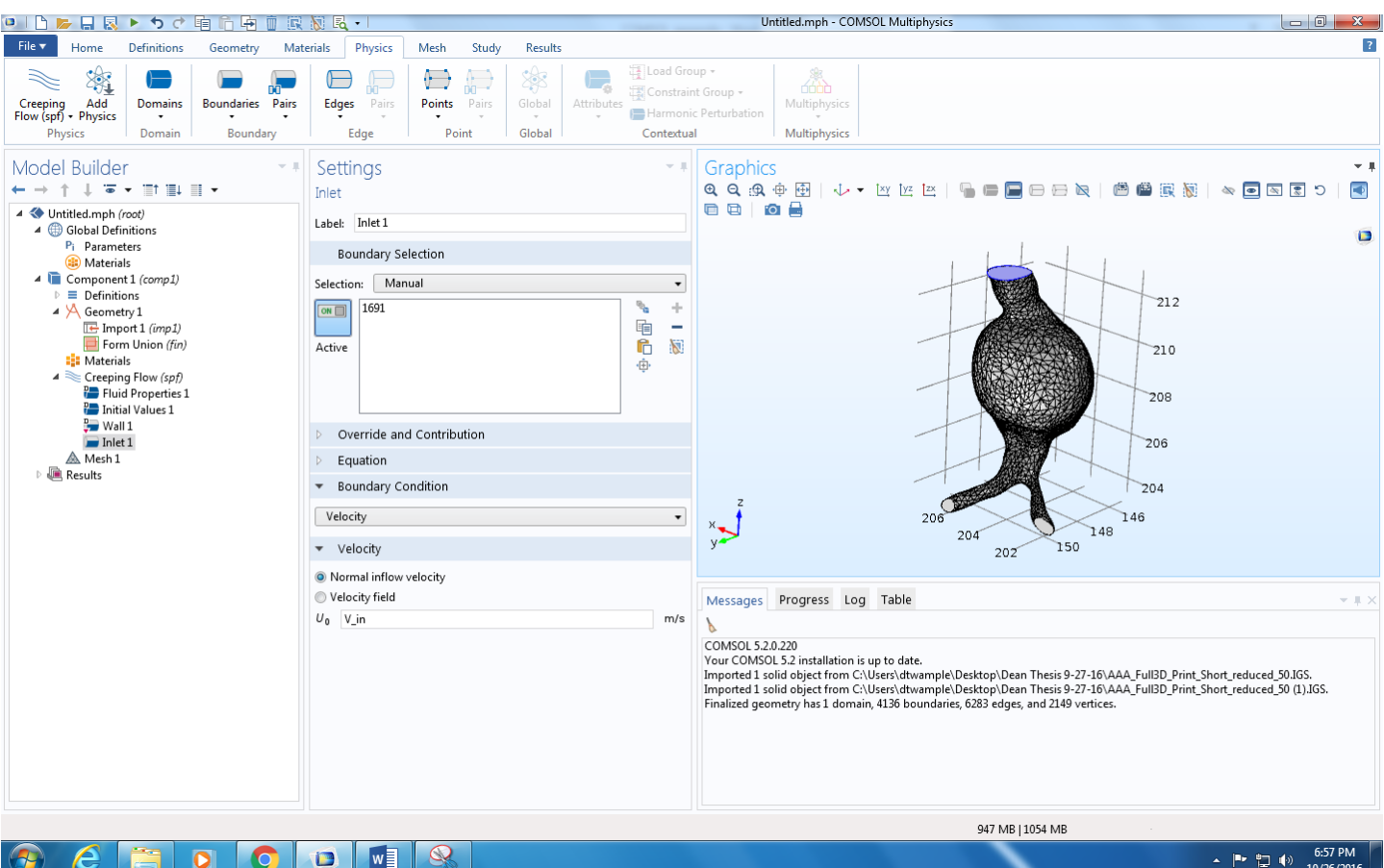

(24) C

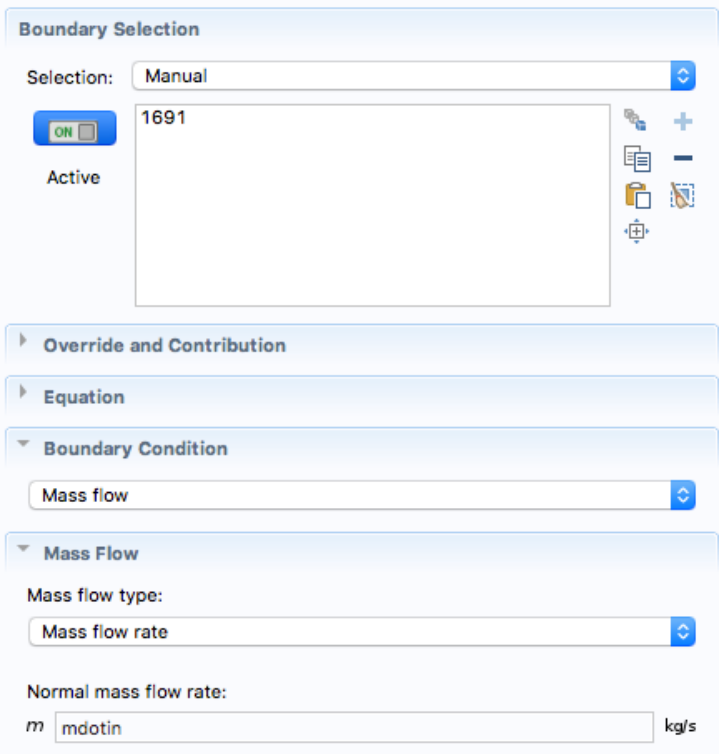




\section{Right click Laminar Flow > Outlet}

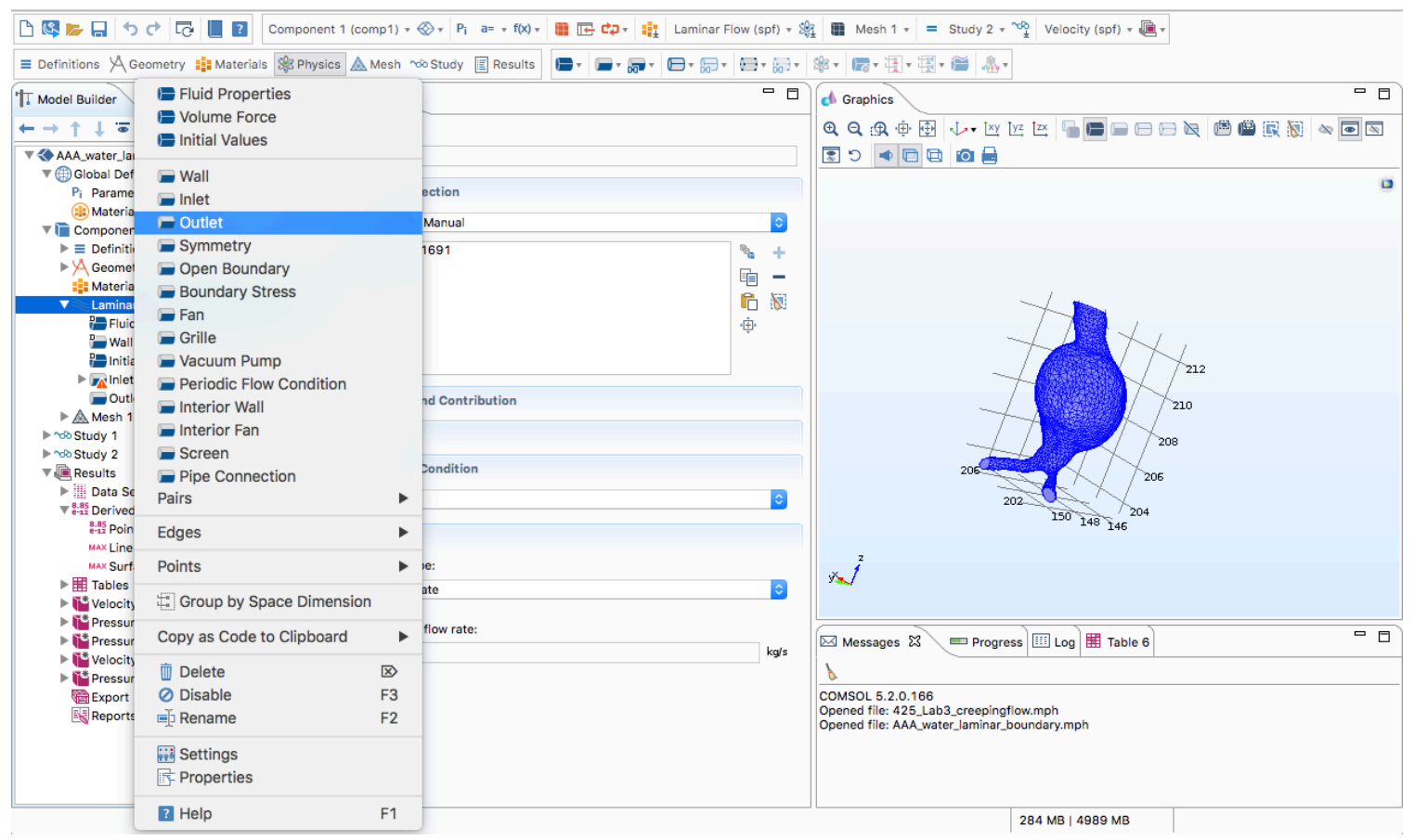


13. Under Boundary Conditions select Pressure. Enter "P_out". Select the exit of both legs of the aneurysm

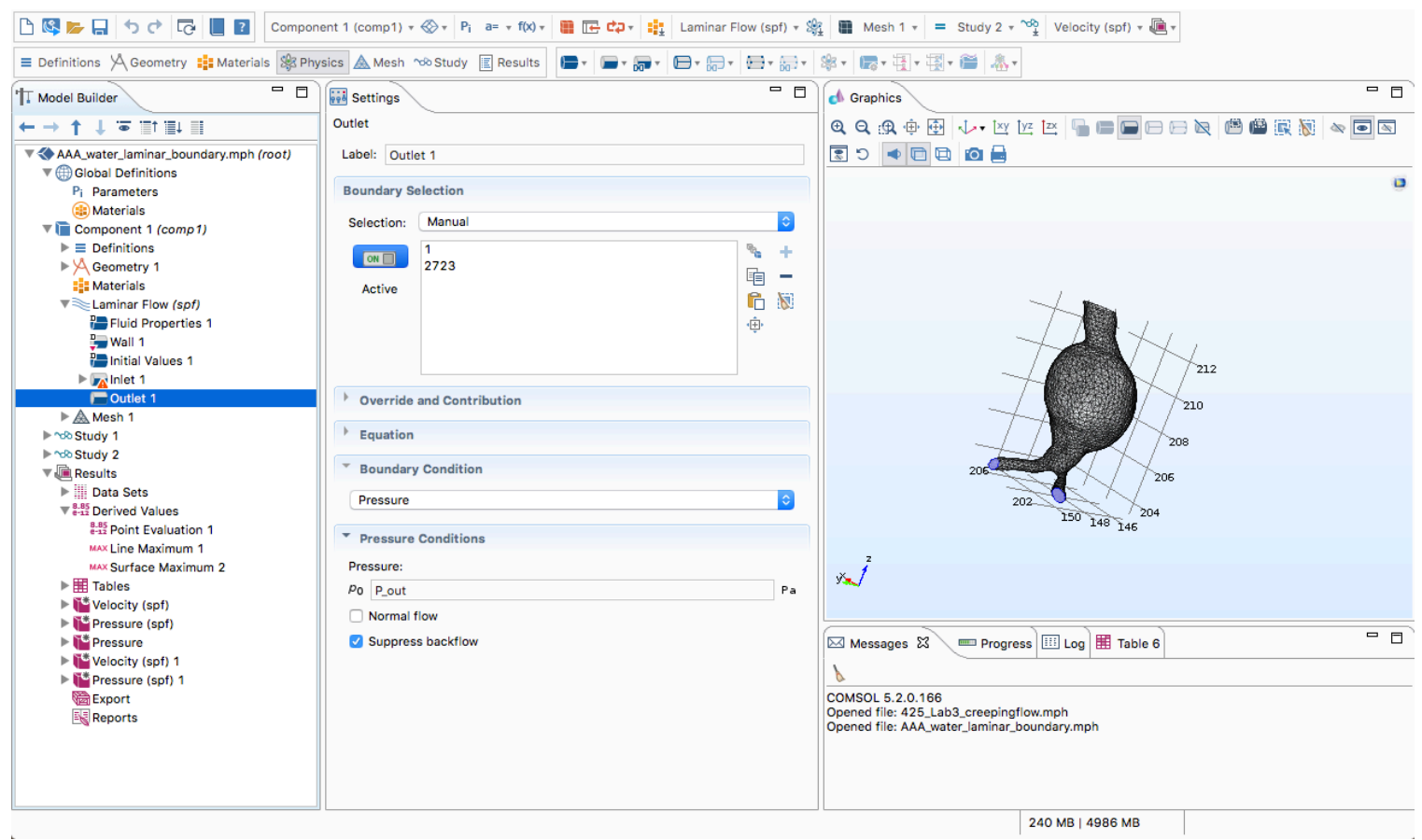

14. Select Initial Conditions. Under Pressure enter "P_out".

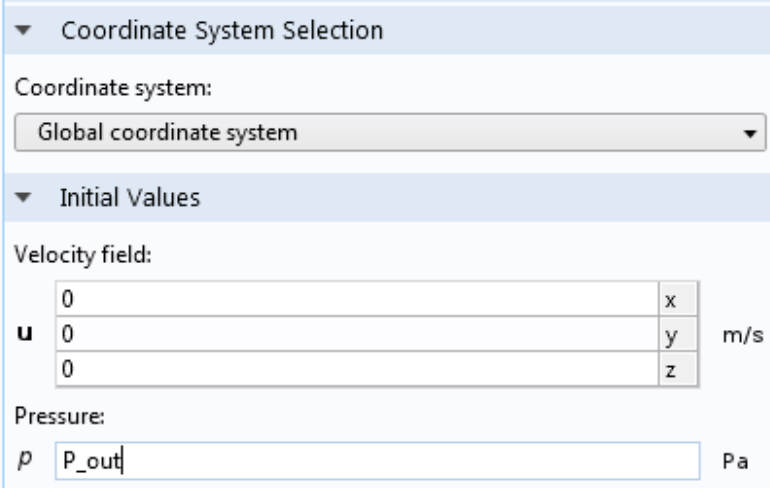


15. Right click Mesh. Select Free Tetrahedral

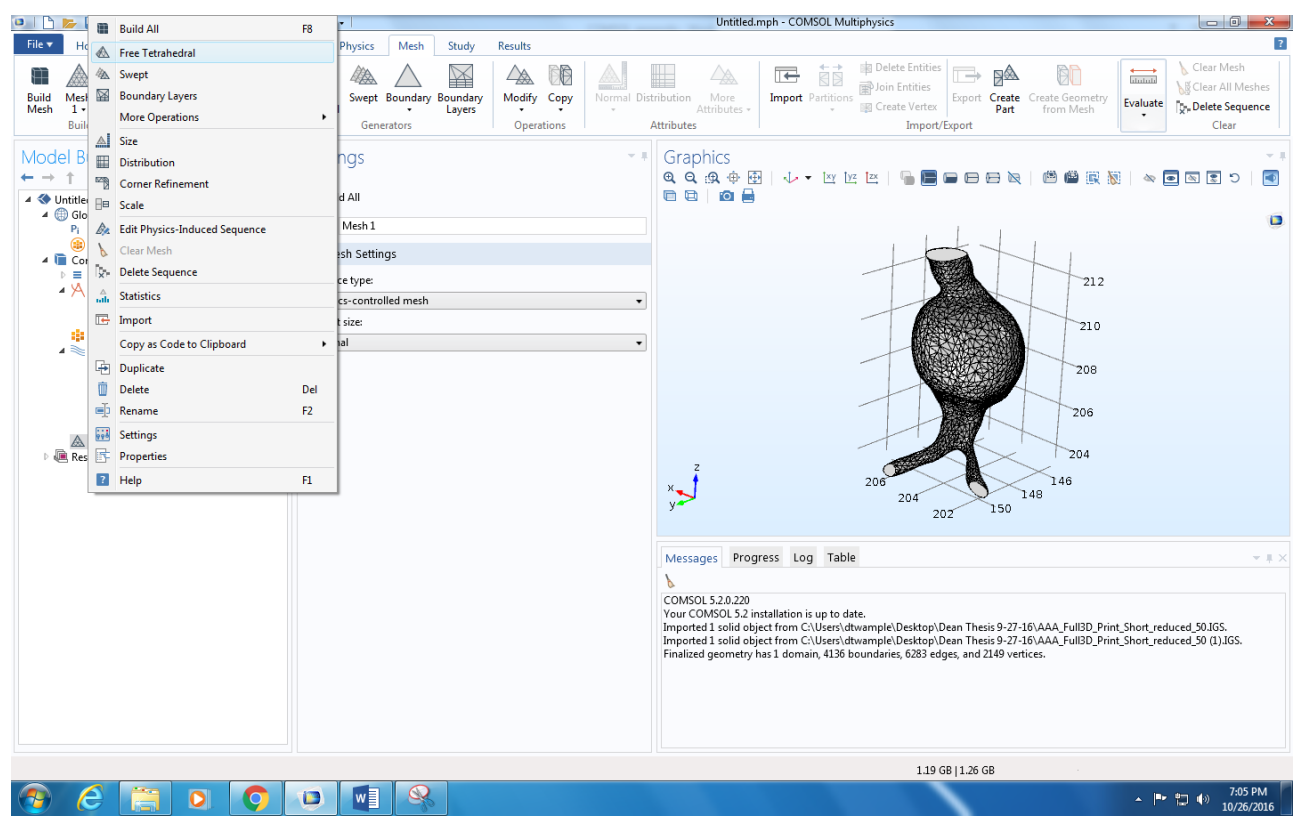


16. Right click on Mesh and select Corner Refinement

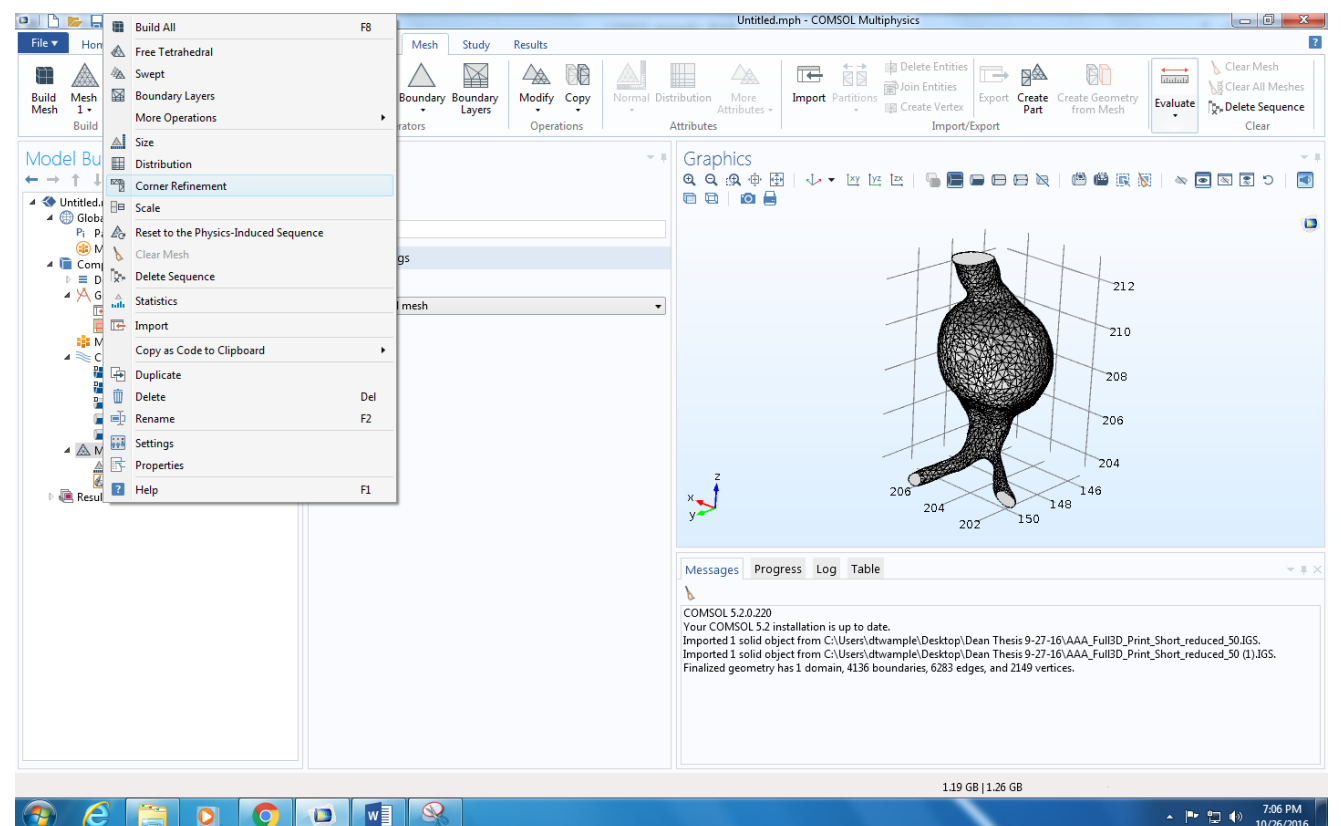




\section{Right click on Mesh and select Boundary Layers}

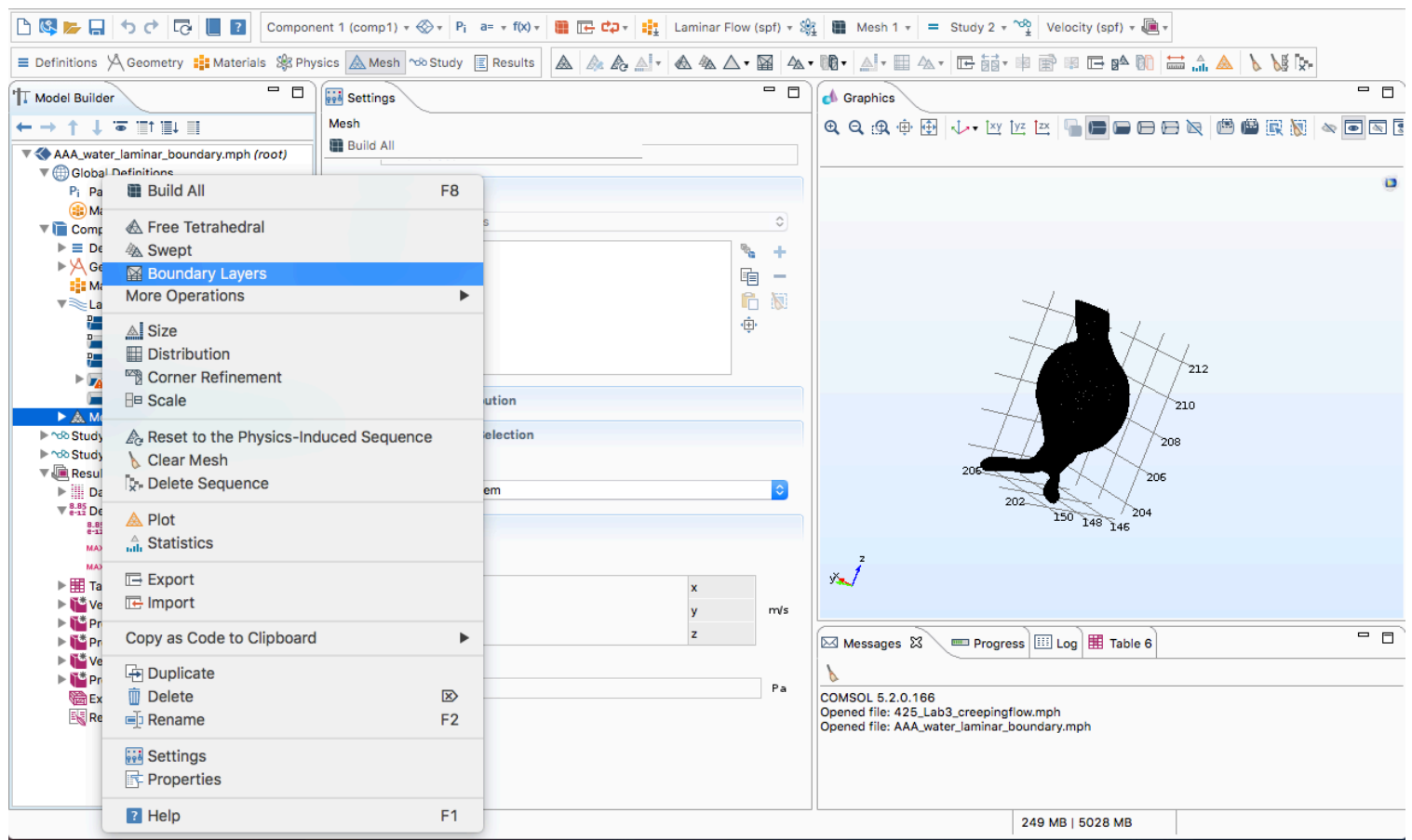

18. Select Size underneath Mesh. Under Calibrate For: Choose General Physics.

Select Fine.

Element Size
Calibrate for:
General physics
Predefined Fine
Custom


19. Select Study $>$ Add Study

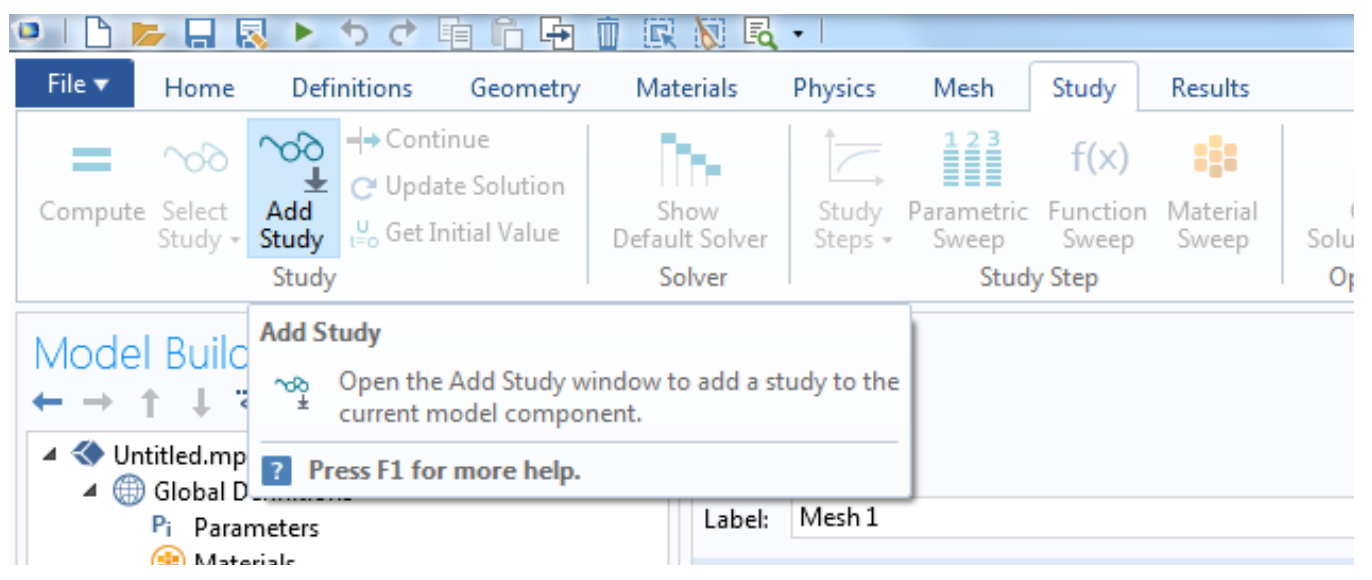

20. Under Study, select Stationary, and choose Add Study

\begin{tabular}{|l|l|}
\hline Add Study \\
\hline Add Study \\
\hline Studies \\
\hline$\infty$ Preset Studies \\
Stationary \\
$\infty \infty$ Custom Studies \\
$\infty$ Empty Study \\
\hline Physics interfaces in study \\
\hline Physics \\
\hline
\end{tabular}


21. Right clock Study and select Compute

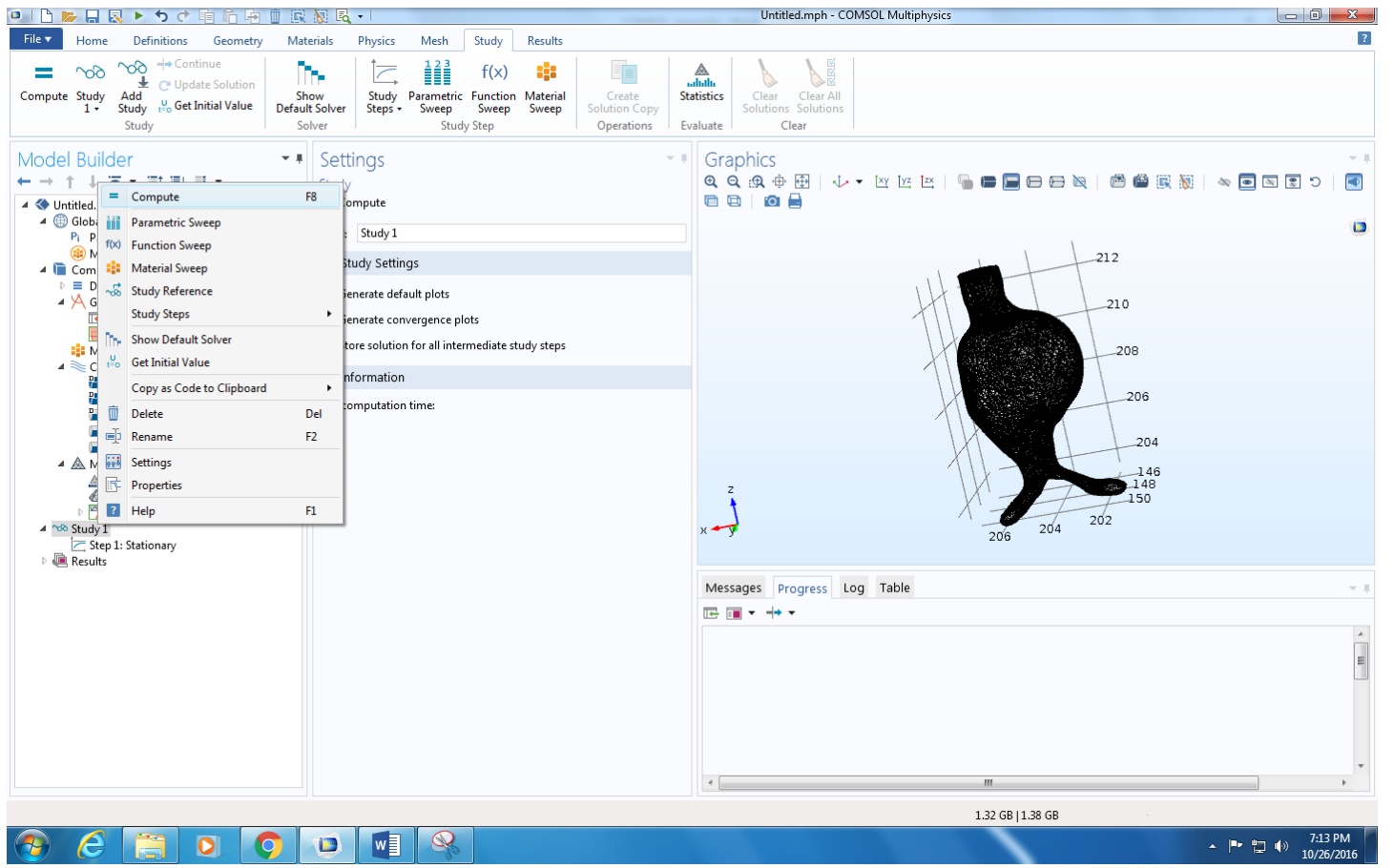


Appendix L: Mesh Statistics for COMSOL Multiphysics ${ }^{\circledR}$ validation study

Normal

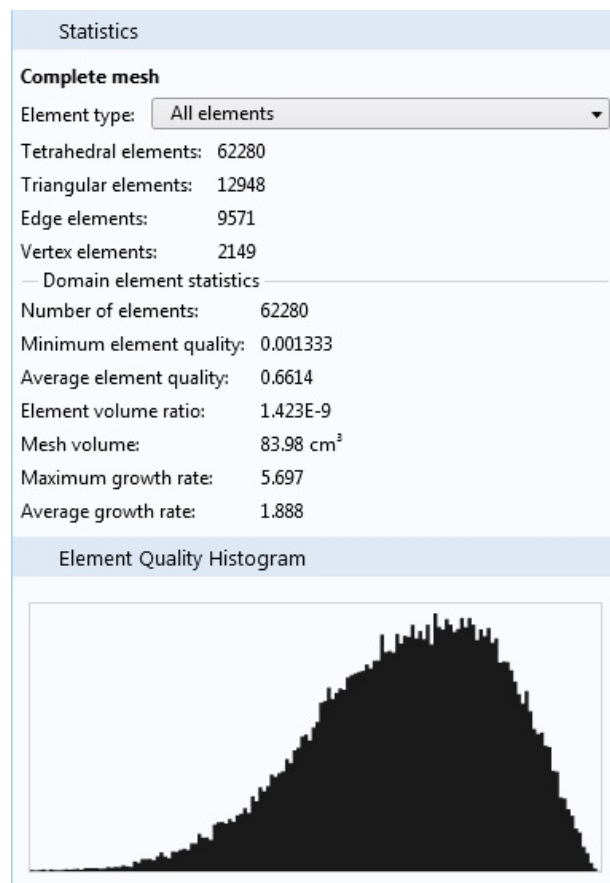

Finer

Statistics
Complete mesh
Element type: All elements
Tetrahedral elements: 279287
Triangular elements: 44072
Edge elements: $\quad 19492$
Vertex elements: $\quad 2149$
Domain element statistics
Number of elements: 279287
Minimum element quality: $\quad 0.001652$
Average element quality: $\quad 0.7046$
Element volume ratio: $\quad 2.115 \mathrm{E}-8$
Mesh volume:
Maximum growth rate:

Fine

Statistics
Complete mesh
Element type: All elements
Tetrahedral elements: 162745
Triangular elements: 28696
Edge elements: $\quad 15250$
Vertex elements: $\quad 2149$
Domain element statistics
Number of elements: 162745
Minimum element quality: $9.576 \mathrm{E}-4$
Average element quality: $\quad 0.6896$
Element volume ratio: $\quad 2.58 \mathrm{E}-9$
Mesh volume:

